\title{
A Novel Millimeter-Wave Dual-Fed Phased Array for Beam Steering
}

\author{
Zur Erlangung des akademischen Grades eines
}

\section{DOKTOR-INGENIEURS}

von der Fakultät für

Elektrotechnik und Informationstechnik, am Karlsruher Institut für Technologie (KIT)

genehmigte

\section{DISSERTATION}

von

\section{M.Sc. Ali Eray Topak \\ geb. in Denizli, Türkei}





\section{Abstract}

A phased array antenna, used for shaping and steering the main antenna beam electronically to chosen directions within the predefined field of view, has been the key antenna system for satellite communications and military radars for decades. However, despite its high functional performance, it is still a very costly and complex solution for emerging wireless consumer applications such as high speed wireless communication and driving assistance systems due to the number of phase shifters and their complex control circuitry. Even more challenges are encountered with an increase in the number of channels if an antenna with high directivity is desired, such as routing RF and IF circuits, isolation of neighboring RF channels or calibration of a whole system.

In order to eliminate the challenges stated above, a novel beam steering approach is presented in this dissertation based on the superposition of two squinted antenna beams. The two antenna beams are realized by exciting the opposite feeds of a dual-fed array antenna. A change in the phase difference and amplitude ratio between the input signals, using only one phase shifter and two variable gain amplifiers or only two I-Q vector modulators, steers the main beam in different directions. Due to its similar architecture, it exhibits all the advantages that a traveling wave antenna possesses as well, such as beam steering with the change of the operating frequency.

Additionally, the sum and difference patterns can be obtained using this concept, allowing for an amplitude-comparison monopulse operation with a broad peak or a deep null at the broadside. Using this approach, beam nulls can also be steered towards interference directions, while keeping the shape and direction of the main beam unchanged. Another advantage is its high robustness against phase and amplitude errors due to analog hardware components in the RF path, which cannot be avoided in conventional phased arrays. A channel mismatch and crosstalk between neighbor RF channels or a temperature and time dependent array calibration can be minimized via this technique thanks to its unique topology, as well.

In this work, an analytic antenna model has been derived and implemented in MATLAB using closed form expressions to analyze and optimize each relevant antenna parameter. Using this model, the development time becomes significantly shorter and the required computer memory is almost negligible.

In order to prove the validity of the proposed novel beam steering approach, two different millimeter-wave (mm-wave) dual-feed antenna setups have been designed and implemented. In the first setup, commercial passive WR-10 components are used to perform the phase and 
amplitude changes manually. To demonstrate the full capability of electronic scanning, a second setup is built by employing an I-Q vector modulator instead of the waveguide phase shifter and the attenuator, which is controllable via input current signals. A mm-wave baredie transceiver MMIC which houses an integrated I-Q vector modulator in its transmitter path has been characterized. For the transition of mm-wave signals from waveguide to planar RF board, a low-loss and broadband waveguide to microstrip line transition has been designed and realized.

The measurement results of both setups show a beam scanning range of $16^{\circ}$ using the phase shifter and an attenuator, well in accord with the simulation results. A monopulse antenna system has been realized for the sum and difference patterns using each test setup. Also, beam steering capability of the both setups has been demonstrated by changing the operating frequency. Even though the test setups have been realized for $77 \mathrm{GHz}$, this technique is applicable at any frequency range. 


\section{Zusammenfassung}

Phasengesteuerte Gruppenantennen (Phased Array Antennen), verwendet für die elektronische Formung und Ausrichtung der Antennen-Abstrahlcharakteristik bzw. des Antennenhauptstrahls innerhalb des vorgegebenen Sichtfeldes, sind seit Jahrzehnten eine Schlüsseltechnologie in der Satelliten-Kommunikation und bei militärischen Radaranlagen. Doch trotz ihrer hohen funktionalen Leistungsfähigkeit, sind sie immer noch eine sehr kostspielige und komplexe Lösung für neu entstehende drahtlose Anwendungen im Bereich der schnellen Datenübertragung oder der Fahrerassistenz-Systeme, bedingt durch die benötigte große Zahl von Phasenschiebern und deren komplexe Ansteuerung bei diesem Konzept. Noch mehr Herausforderungen ergeben sich mit der Erhöhung der Anzahl der Kanäle, wenn eine Antenne mit starken Richtwirkung erwünscht wird, zum Beispiel bei der Verdrahtung von integrierte HF und ZF-Bausteinen, bei der Isolation benachbarter HF-Kanäle oder bei der Kalibration der gesamten Systemanordnung.

Zur Bewältigung der oben aufgeführten Herausforderungen wird in der vorliegenden Dissertation ein neues Verfahren für die Formung und Ausrichtung der Antennenkeulen vorgestellt, basierend auf der Superposition zweier gegen die Antennennormale gedrehte Antennenstrahlen. Die beiden Antennenkeulen werden durch die gleichzeitige Anregung der Einspeisepunkte einer zweiseitig einspeisbaren linearen Gruppenantenne (Dual Fed Antenne) erzeugt. Eine Änderung der Phasendifferenz und des Amplitudenverhältnisses zwischen den beiden Anregungssignal mit Hilfe eines Phasenschiebers und eines Verstärkers mit variabel einstellbarer Verstärkung, oder stattdessen nur eines IQ-Vektor-Modulators, steuert den Hauptstrahl in verschieden Richtungen. Aufgrund der Ähnlichkeit des Aufbaus mit dem einer Wanderwellenantenne (Traveling Wave Antennas), besitzt er alle Vorteile solcher Wanderwellenantennen, wie zum Beispiel die Steuerung der Hauptkeule mit der Betriebsfrequenz.

Zusätzlich lässt sich das Summen- und Differenz-Antennendiagramm durch die Verwendung dieses Konzepts bestimmen, die einen amplitudenvergleichenden Monopulsbetrieb mit einem breiten Hauptstrahl oder einer Auslöschung in Hauptrichtung der Antenne ermöglichen. Mit diesem Verfahren können auch Nullstellen im Antennendiagramm in Richtungen von Störern gelegt werden, während die Form und Richtung der Hauptkeule erhalten bleibt. Ein weitere Vorteil ist die Robustheit des Verfahrens gegenüber von Phasen- und Amplitudenfehlern die von der analogen Hardware im HF-Pfad kommen, welche bei konventionellen phasengesteuerten Gruppenantennen nicht vermieden werden können. Durch das beschriebene Verfahren können eine Kanal-Fehlanpassung und ein Übersprechen zwischen benachbarten HFKanälen verringert werden oder auch eine temperaturabhängige und zeitabhängige Kalibration vermieden werden. In der vorgelegten Arbeit wurde ein analytisches Antennenmodell 
mit geschlossenen Ausrücken entwickelt und in MATLAB implementiert, mit dem jeder relevante Parameter der Antenne analysiert und optimiert werden kann. Mit diesem Modell lässt sich die Entwicklungszeit wesentlich reduzieren und der benötigte Computer-Speicher ist nahezu vernachlässigbar.

Um die Gültigkeit des neuen Steuerverfahrens des Antennendiagramms unter Beweis zu stellen, wurden zwei verschiedene Dual-Fed Antennen für Millimeter-Wellen entworfen und implementiert. Im ersten Aufbau werden kommerzielle passive WR-10 HohlleiterKomponenten benutzt, um die Phasen- und Amplitudenverschiebungen manuell vorzunehmen. Um die volle Leistungsfähigkeit der elektronischen Abtastung zu demonstrieren, wird ein zweiter Aufbau realisiert, bei dem anstelle des Hohlleiter-Phasenschiebers- und Dämpfungsgliedes ein IQ-Vektor-Modulator benutzt wird, der durch Eingangsstromsignale kontrollierbar ist. Ein nicht gehauster integrierter Millimeterwellen Transceiver Chip (Transceiver MMIC), der einen IQ-Vektor-Modulator in seinem Sendepfad beherbergt, wurde charakterisiert. Für die Transmission des Millimeterwellen-Signals vom Hohlleiter zur planaren Hochfrequenz-Platine wurde ein verlustarmer und breitbandiger Übergang vom Hohlleiter auf eine Mikrostreifenleitung entworfen und realisiert.

Die Messergebnisse für beide Aufbauten zeigen einen Steuerungsbereich des Hauptstrahls über $16^{\circ}$, wenn der Phasenschieber und das Dämpfungsglied benutzt werden, in guter Übereinstimmung mit den Resultaten aus der Simulation. Ein Monopuls-Antennensystem zur Erzeugung des Summen- und Differenz-Antennendiagramms wurde mit beiden TestAnordnungen realisiert. Ebenso wurde die Strahlsteuerung durch Änderung der Betriebsfrequenz mit beiden Aufbauten bewiesen. Auch wenn die Test-Systeme für $77 \mathrm{GHz}$ aufgebaut wurden, so ist diese Technik doch für jeden beliebigen Frequenzbereich anwendbar. 


\section{Acknowledgments}

I would like to sincerely thank Prof. Dr.-Ing. Thomas Zwick, the director of Institut für Hochfrequenztechnik und Elektronik (IHE) at the Karlsruhe Institute of Technology, for his invaluable guidance and support during my work. Next, I would like to give my sincere thanks to Prof. Dr.-Ing. Christian Waldschmidt, the co-examiner and the director of Institute of Microwave Techniques at the University of Ulm for his careful review of my Ph.D. thesis.

Particularly, I would like to express my deepest appreciation to my advisor, Dr. Jürgen Hasch from the Robert Bosch $\mathrm{GmbH}$, who has been a constant source of encouragement and support.

I am also grateful to my colleagues from CR/ARE1 and CC-DA/ECR1 at Robert Bosch $\mathrm{GmbH}$ who assisted me during my Ph.D. work. I am particularly indebted to Uwe Wostradowski, Jürgen Seidel, Stefan Gaier, Prof. Dr.-Ing. Matthias Hampe, Joachim Selinger and Dr. Stefan Schulteis for the valuable suggestions and their technical assistance during early part of my Ph.D. I am grateful to Dr. Thomas Fritzsche for taking the time to regularly discuss the progress of my work. I would like to thank to Delf Mittelstraß for the support during measurements and valuable discussions. The thesis would not be possible without the assistance of Dr. Rudolf Lachner and Dr. Christoph Wagner from Infineon Technologies who provided SiGe MMICs. Financial and equipment grant support received from the German Federal Ministry of Education and Research (BMBF) under Contract 13N9820-13N9824 Radar on Chip for Cars (RoCC) are gratefully acknowledged.

I am thankful to Serdal Ayhan, Mekdes Girma, Manuel Dudek, Dr. Markus Gonser, Dr. Thomas Merkle, Onur Ucaner, Furkan Dayi, Dr. Ömer Bulakci, Can Uz, Mustafa Sarpasan and Volkan Öztürk for their selfless help and support during my hard times.

Finally, I would like to thank my parents, my brother, and my girlfriend, Katrin. Their encouragement, care, and support provided me with the energy I need to pursuit my Ph.D. degree. 
- vi - 


\section{Contents}

Abstract

Zusammenfassung iii

Acknowledgments $\quad$ V

List of Abbreviations $\quad$ ix

List of Symbols $\quad x$

1. Introduction 1

1.1. Conventional phased arrays . . . . . . . . . . . . . . 3

1.2. Drawbacks of conventional phased arrays . . . . . . . . . . . . 7

1.3. A novel phased array approach . . . . . . . . . . . . . . 8

1.4. Dissertation overview . . . . . . . . . . . . . . . . . . . 9

2. A Novel Phased Array Concept 11

2.1. Array theory . . . . . . . . . . . . . . . . . . . 11

2.2. Dual-fed phased array demonstrator employing a planar array . . . . . . . . 13

2.2.1. Planar array . . . . . . . . . . . . . . . . 13

2.2.2. Simulation and Optimization . . . . . . . . . . . . . . . 14

3. Analytical Antenna Model 23

3.1. Introduction . . . . . . . . . . . . . . . . . 23

3.2. Analytical model for a mm-wave microstrip linear array . . . . . . . . . . . 25

3.2.1. Computation of electrical parameters . . . . . . . . . . . 25

3.2.2. Analysis of a straight microstrip line . . . . . . . . . . . . . 28

3.2.3. Analysis of a single patch antenna including feed lines . . . . . . . . 29

3.2.4. Analysis of a microstrip linear array antenna . . . . . . . . . . 32

3.2.5. Dual-fed linear array antenna . . . . . . . . . . . . . . . 35

3.3. Investigation of design parameters . . . . . . . . . . . . . . . 40

3.3.1. Number of patch elements . . . . . . . . . . . . . . . . . . . . . 40

3.3.2. Length of feed lines between patches . . . . . . . . . . . . . . . . . . . . . . . . . 42

3.3.3. Operating frequency . . . . . . . . . . . . . . 42

4. Measurement Setups 49

4.1. Overview of measurement setups . . . . . . . . . . . . . . . . . . . 49 
4.2. Waveguide setup . . . . . . . . . . . . . . . . 53

4.3. A 77-GHz SiGe MMIC I-Q vector modulator . . . . . . . . . . . . 55

4.3.1. Architecture . . . . . . . . . . . . . . . . 55

4.3.2. Test setup . . . . . . . . . . . . . . . . 57

4.3.3. Measurement results . . . . . . . . . . . . . . . 60

4.4. A millimeter-wave waveguide to microstrip line transition . . . . . . . 62

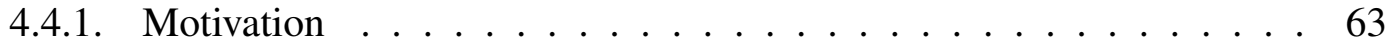

4.4.2. Design Procedure . . . . . . . . . . . . . . . . . . . . . 64

4.4.3. Assembly and Measurements . . . . . . . . . . . . . 67

4.5. Millimeter-wave planar balun . . . . . . . . . . . . . . . . . . . . 69

5. Test and Measurements $\quad \mathbf{7 1}$

5.1. Setup based on passive WR-10 attenuator and phase shifter . . . . . . . . 71

5.1.1. Test configuration . . . . . . . . . . . . . . . 71

5.1.2. Antenna with amplitude tapering . . . . . . . . . . . . . 74

5.1.3. Measurement results . . . . . . . . . . . . . . . . 74

5.2. Setup based on active MMIC I-Q vector modulator $\ldots \ldots \ldots \ldots$. . . 80

5.2.1. Test configuration . . . . . . . . . . . . . . . . 80

5.2.2. Antenna without amplitude tapering . . . . . . . . . . 81

5.2.3. Measurement results . . . . . . . . . . . . . . . . . . . . 83

6. Conclusion and Future Work 89

6.1. Summary and Conclusion . . . . . . . . . . . . . . . . . . . 89

6.2. Future Work . . . . . . . . . . . . . . . . . . . . . . . . . . . 91

$\begin{array}{ll}\text { Bibliography } & 93\end{array}$

$\begin{array}{ll}\text { A. Appendix } & 99\end{array}$ 


\section{List of Abbreviations}

$\begin{array}{ll}\text { ADS } & \text { Advanced Design System } \\ \text { CMOS } & \text { Complementary Metal Oxide Semiconductor } \\ \text { DAQ } & \text { Data Acquisition } \\ \text { EM } & \text { Electromagnetic } \\ \text { FEM } & \text { Finite Element Method } \\ \text { FDTD } & \text { Finite-Difference Time-Domain } \\ \text { GPIB } & \text { General Purpose Interface Bus } \\ \text { HBT } & \text { Heterojunction Bipolar Transistor } \\ \text { HPBW } & \text { Half-Power Beamwidth } \\ \text { IF } & \text { Intermediate Frequency } \\ \text { ISM } & \text { Industrial, Scientific and Medical } \\ \text { I-Q } & \text { In-phase and Quadrature } \\ \text { LabVIEW } & \text { Laboratory Virtual Instrumentation Engineering Workbench } \\ \text { LO } & \text { Local Oscillator } \\ \text { mm-wave } & \text { Millimeter-wave } \\ \text { MoM } & \text { Method of Moments } \\ \text { MMIC } & \text { Monolithic Microwave Integrated Circuit } \\ \text { PCB } & \text { Printed Circuit Board } \\ \text { POM } & \text { Polyoxymethylene } \\ \text { RF } & \text { Radio Frequency } \\ \text { SiGe } & \text { Silicon Germanium } \\ \text { SLL } & \text { Sidelobe levels } \\ \text { SNR } & \text { Signal to Noise Ratio } \\ \text { TE } & \text { Transverse Electric } \\ \text { TEM } & \text { Transverse Electromagnetic } \\ \text { TM } & \text { Transverse Magnetic }\end{array}$




\section{List of Symbols}

$\begin{array}{ll}\theta & \text { Direction of a main beam } \\ f & \text { Operating frequency } \\ h & \text { Height of a dielectric substrate } \\ \epsilon_{r} & \text { Relative dielectric permittivity } \\ \epsilon_{e f f} & \text { Effective dielectric permittivity } \\ L_{f} & \text { Length of a feed line between subsequent patch elements } \\ L_{p} & \text { Length of a patch element } \\ t & \text { Thickness of a conductor line } \\ A_{1} & \text { Input value fed into port } 1 \\ A_{2} & \text { Input value fed into port } 2 \\ w & \text { Width of a microstrip line } \\ W_{p} & \text { Width of a patch element } \\ W_{f} & \text { Width of a feed line } \\ \lambda_{g} & \text { Guided wavelength } \\ \lambda_{0} & \text { Free space wavelength } \\ \phi_{1} & \text { Input phase fed into port } 1\left(^{\circ}\right) \\ \phi_{2} & \text { Input phase fed into port } 2\left(^{\circ}\right) \\ \Delta A & A_{2} / A_{1} \text { Amplitude ratio }(\text { linear scale) } \\ \Delta A_{d B} & 20 \text { log }\left(A_{2} / A_{1}\right) \text { Amplitude ratio (logarithmic scale) } \\ \Delta \phi & \phi_{2}-\phi_{1} \text { Phase difference }\end{array}$




\section{List of Figures}

1.1. Automotive radar applications. . . . . . . . . . . . . . . . . 2

1.2. High speed wireless data communication (Courtesy of Wireless Gigabit Al-

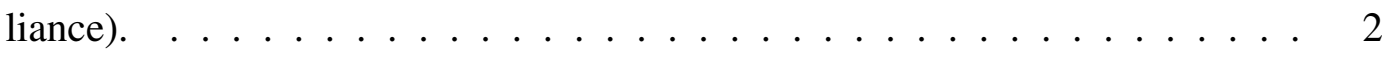

1.3. A conventional phased array configuration. . . . . . . . . . . 3

1.4. A typical antenna pattern $(N=8, f=77 \mathrm{GHz}, \Delta \phi=0, d=0.5 \lambda) \ldots \ldots . . . . .4$

1.5. Radiation patterns with different amplitude tapering methods $(N=8, f=77$ $\mathrm{GHz}, \Delta \phi=0, d=0.5 \lambda) \ldots \ldots \ldots \ldots \ldots \ldots$

1.6. Array factors (AF) for antenna arrays with $N=2, N=6, N=10$ and $N=14$ elements $(d=0.5 \lambda, f=77 \mathrm{GHz}, \Delta \phi=0) \ldots \ldots \ldots \ldots$

1.7. Array factors (AF) for antenna arrays with $d=0.25 \lambda, d=0.5 \lambda, d=0.75 \lambda$ and $d=\lambda$ element spacings $(N=4, f=77 \mathrm{GHz}, \Delta \phi=0) . \ldots \ldots \ldots 6$

1.8. Array factors (AF) for antenna arrays with $\Delta \phi=-0.75 \pi, \Delta \phi=-0.25 \pi, \Delta \phi=0.25 \pi$ and $\Delta \phi=0.75 \pi$ phase values $(N=4, f=77 \mathrm{GHz}, d=0.5 \lambda) \ldots \ldots \ldots$

1.9. Vertical misalignment of a radar sensor (Courtesy of BMW). . . . . . . . . 9

1.10. Beam steering in elevation to detect a bridge. . . . . . . . . . . . 9

2.1. A traveling wave antenna and resulting beam / wave front. . . . . . . . . 11

2.2. Working principle of the dual-fed phased array, feeding a linear array at (a) only Port 1, (b) only Port 2, (c) Ports $1 \& 2 \ldots \ldots$. . . . . . . . . 12

2.3. Linear array using series-fed patch antenna elements. . . . . . . . . . . . 12

2.4. Direction of the main beam for $L_{f}=\lambda_{g} / 2\left(L_{p}=\lambda_{g} / 2\right) \ldots \ldots \ldots$. . . . . . . . 14

2.5. Direction of the main beam based on the feed line length $\left(L_{f}\right)$ between patches

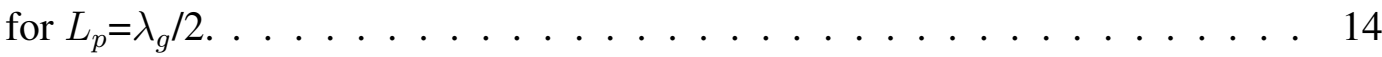

2.6. Beam steering by change of the operating frequency from 75 to $81 \mathrm{GHz}$. . . 15

2.7. Beam steering using a phase shifter and an attenuator $\left(L_{f}=0.85 \mathrm{~mm}, N=5\right)$ (Simulation). . . . . . . . . . . . . . . . 16

2.8. Sum $(\Sigma) /$ difference $(\Delta)$ antenna patterns which are the solid and dashed curves respectively $\left(L_{f}=1.5 \mathrm{~mm}, N=5\right)$ (Simulation) $\ldots \ldots \ldots \ldots$

2.9. Effect of amplitude and phase errors on a sum antenna pattern $\left(L_{f}=1.5 \mathrm{~mm}\right.$, $N=5$ ) (Simulation). . . . . . . . . . . . . . . . . . . 18

2.10. Comparison of continuous and discrete beam scans employing same feed line lengths $\left(L_{f}\right)$ and different number of antenna elements $(N) \ldots \ldots \ldots$. . . . 19

2.11. Equivalent model of the linear patch array. . . . . . . . . . . . . 20

2.12. An example for a dual-fed microstrip array antenna. . . . . . . . . . . . . 21

2.13. An example for a dual-fed slotted waveguide array antenna. . . . . . . . . . 21 
2.14. An example for a dual-fed microstrip planar array antenna. A narrow beam can be obtained in both azimuth and elevation. . . . . . . . . . . . . . . 22

3.1. The design flow. . . . . . . . . . . . . . . . . . . . 24

3.2. A circuit model of a microstrip line. . . . . . . . . . . . . . . 28

3.3. Amplitude and phase errors of analytical model compared to full-wave simulation using straight microstrip lines with different widths $(w)$, as shown in Fig. 3.2. . . . . . . . . . . . . . . . . . . 30

3.4. A single patch antenna interconnected with feed lines. . . . . . . . . . . 31

3.5. A microstrip step discontinuity and its equivalent circuit. . . . . . . . . . . 32

3.6. Amplitude and phase errors of analytical model compared to the full-wave EM simulation using patch elements with different patch widths $\left(w_{p}\right)$ connected with feed lines, as shown in Fig. $3.4\left(L_{f}=5 \mathrm{~mm}\right) \ldots \ldots . . . . . .33$

3.7. Rectangular microstrip resonator antenna. . . . . . . . . . . . . . . . . 34

3.8. Effect of feed line length on the radiation pattern of a linear array antenna (with $N=10$ ). The antenna is fed by one port only. . . . . . . . . . . 37

3.9. Effect of feed line length on the radiation pattern of a linear array antenna (with $N=5$ ). The antenna is fed by one port only. . . . . . . . . . . . 38

3.10. Analytical model of a linear array antenna fed by both ports. . . . . . . . . . 39

3.11. Dual-fed linear array antenna consisting up of 10 patch elements $\left(L_{f}=1.25\right.$ mm) . . . . . . . . . . . . . . . . . . . 39

3.12. Effect of the number of antenna elements on the radiation pattern of a linear array antenna $\left(L_{f}=1.5 \mathrm{~mm}\right) . \ldots \ldots \ldots . \ldots . \ldots . \ldots . \ldots 44$

3.13. Effect of the number of antenna elements on the radiation pattern of a linear array antenna $\left(L_{f}=1.25 \mathrm{~mm}\right) . \ldots \ldots \ldots . \ldots . \ldots . \ldots 45$

3.14. Effect of feed line length on the radiation pattern of a linear array antenna $(N=10) \ldots \ldots \ldots \ldots \ldots \ldots \ldots$. . . . . . . . . . . . . . . . . .

3.15. Effect of the operating frequency on the radiation pattern of a linear array antenna $\left(N=5\right.$ and $\left.L_{f}=0.8 \mathrm{~mm}\right) . \ldots \ldots \ldots \ldots \ldots$

4.1. System architectures of two different dual-fed antenna setups: (a) Setup employing passive waveguide phase shifter and variable attenuator, (b) Setup employing an active MMIC I-Q vector modulator. . . . . . . . . . . . 50

4.2. A photograph of the dual-fed setup employing a waveguide phase shifter and variable attenuators: (a) Waveguide components, (b) A planar RF-board (Table A.1) . . . . . . . . . . . . . . . . . . . . 51

4.3. A photograph of the dual-fed setup employing I-Q transceiver MMICs: (a) Waveguide components, (b) A planar RF-board (Table A.1) employing I-Q transceiver MMICs. . . . . . . . . . . . . . . . . . . . . 52

4.4. The waveguide setup used for characterization. . . . . . . . . . . . . . 53

4.5. Amplitude ratios between both channels by adjusting the variable attenuators. 54

4.6. Phase differences between both channels by adjusting the phase shifter at Port 2. 54

4.7. An I-Q transceiver chip. . . . . . . . . . . . . . . . . . . . 56 
4.8. An I-Q vector model. . . . . . . . . . . . . . . . . . . . . . 57

4.9. The block diagram of the measurement system for the I-Q transceiver. . . . . 58

4.10. A control board for the $\mathrm{I}-\mathrm{Q}$ transceiver. . . . . . . . . . . . . . . 59

4.11. RF test board for the I-Q transceiver. . . . . . . . . . . . . . . . . . . . . 59

4.12. Measurement setup for the I-Q transceiver module. . . . . . . . . . . . . . . 60

4.13. Measured transmission characteristics for different phase states. The curve parameter is the phase of the current $\theta=\arctan ((Q-3.5) /(I-3.5))$, while the absolute current $I_{t o t}=\sqrt{(I-3.5)^{2}+(Q-3.5)^{2}}=3.5 \mathrm{~mA}$. . . . 61

4.14. Measured transmission characteristics for different amplitude states. The curve parameter is the current in the I-channel $I$, the current in the Q-channel

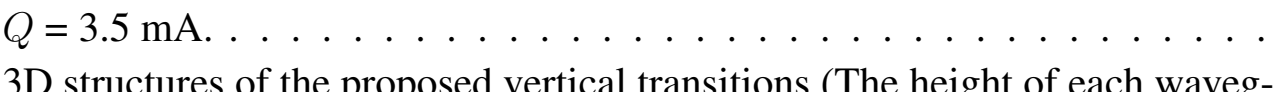

4.15. 3D structures of the proposed vertical transitions (The height of each waveguide flange is $6 \mathrm{~mm}$.): (a) The simple transition without the waveguide iris (Type A), (b) The transition with the waveguide iris (Type B). . . . . . . . . 63

4.16. Cross-sections of two different waveguide transitions. . . . . . . . . . . . . 64

4.17. (a) Top view of the fabricated planar circuit used for the both waveguide transitions, (b) Effect of the parameter $\mathrm{w}_{\text {off }}$ on impedance matching of the transition without iris (Type A). . . . . . . . . . . .

4.18. Simulated S-parameters of the transitions (curve with circle: $S_{11}$ for $\Delta a_{s 1}=+60$ um , curve with diamond: $S_{11}$ for $\Delta a_{s 1}=-60 \mathrm{um}$, curve with square: $S_{11}$ for $\Delta \mathrm{h}_{s 1}=-200 \mathrm{um}$, curve with square: $\mathrm{S}_{11}$ for $\left.\Delta \mathrm{h}_{s 1}=+200 \mathrm{um}\right) \ldots \ldots \ldots \ldots$

4.19. Manufactured RF board including the waveguide flanges: (a) Fabricated RF board for back-to-back assembly, (b) Metallic waveguide flanges connected to the RF board and microstrip lines with coplanar transitions on the same board . . . . . . . . . . . . . . . . 68

4.20. Simulated and measured results of the transition without iris (Type A). . . . 68

4.21. Simulated and measured results of the transition with iris (Type B). . . . . . . 69

4.22. Back to back test setup for baluns connected to each other for characterization. 70

4.23. Measured and simulated insertion and return losses of connected baluns. . . . 70

5.1. Antenna measurement setup employing waveguide phase shifter. . . . . . . . 72

5.2. Measurement platform for setup based on waveguide phase shifter and attenuator. . . . . . . . . . . . . . . . . . . 73

5.3. Characterization of a linear array antenna including waveguide transitions. . . 75

5.4. Sum and difference antenna patterns $\left(A 2=A 1, \Delta \phi=\phi_{2}-\phi_{1}\right) \ldots \ldots$. . . . . 76

5.5. Beam steering using a single phase shifter and attenuator. . . . . . . . . . 77

5.6. H-plane radiation pattern $\left(A 1=A 2=0.707, \Delta \phi=180^{\circ}\right) \ldots \ldots \ldots$. . . . . 78

5.7. Beam steering in E-plane by change of operating frequency ( $76 \mathrm{GHz}$ to 81 $\mathrm{GHz}) \ldots \ldots \ldots \ldots \ldots \ldots \ldots$

5.8. Radiation pattern at different operating frequencies. . . . . . . . . . . . 79

5.9. Antenna measurement setup employing bare-die I-Q vector modulator. . . . . 80

5.10. Realized antenna setup. . . . . . . . . . . . . . . . . 81 
5.11. RF board employing a linear array antenna and MMIC phase shifters on the measurement platform. . . . . . . . . . . . . . 82

5.12. Control boards for I-Q modulators. . . . . . . . . . . . . . . . 82

5.13. Characterization of the linear array antenna made up of 5 patch elements. . . 84

5.14. Sum and difference patterns in the E-plane (Measurement \& Simulation). . . 85

5.15. Beam steering in the E-plane (Measurement \& Simulation). . . . . . . . . . . 85

5.16. H-plane radiation pattern. . . . . . . . . . . . . . . 86

5.17. Beam steering by change of operating frequency ( $\mathrm{f}=75$ to $81 \mathrm{GHz}) \ldots$. . . . 86

5.18. Radiation pattern at different frequencies. . . . . . . . . . . . . 87

A.1. Physical parameters of an RF board . . . . . . . . . . . . . . . . . . . . 99

A.2. Layout of the designed linear array . . . . . . . . . . . . . . . . . . . . . . . . . . . . . . . .

A.3. Data sheet of the RO3003 dielectric substrate . . . . . . . . . . . . . . . . 101 


\section{List of Tables}

2.1. Optimized physical parameters of rectangular patches used for a 77-GHz linear array (Fig. A.2) . . . . . . . . . . . . . . . . . . . 16

2.2. Change of number of patch elements $(N)$ at $77 \mathrm{GHz} . \ldots \ldots 20$

2.3. Change of length of feed lines $\left(L_{f}\right)$ at $77 \mathrm{GHz} \ldots \ldots \ldots$

3.1. Effect of number of patch elements on an overall pattern $\left(L_{f}=1.5 \mathrm{~mm}\right)$. . . 41

3.2. Effect of number of patch elements on an overall pattern $\left(L_{f}=1.25 \mathrm{~mm}\right)$. . . 41

3.3. Effect of feed line length on an overall pattern $(N=10) \ldots \ldots \ldots$. . . . . . 42

3.4. Effect of an operating frequency on an overall pattern $\left(N=5, L_{f}=0.8 \mathrm{~mm}\right)$. . 43

5.1. Parameters of a dual fed array design with amplitude tapering at $77 \mathrm{GHz}$. . 74

5.2. Parameters of a dual fed array design without amplitude tapering at $77 \mathrm{GHz}$. $\quad 83$

5.3. Comparison between the dual-fed phased array architectures. . . . . . . . . 88

6.1. Comparison between the conventional and dual-fed phased array architectures. 91

A.1. Dimensions of a planar RF circuit on a RO3003 substrate . . . . . . . . . . . 99

A.2. Optimized physical parameters of rectangular patches used for a linear array

(Fig. A.2) . . . . . . . . . . . . . . . . . . . 100 


\section{Introduction}

Phased arrays have become the major antenna system for satellite communications and military systems in the last decades since they allow the electronic steering of a main antenna beam to any direction within the predefined field of view with high antenna gain and almost negligible signal processing power [1], [2]. The beam steering is done by controlling phase and amplitude of each antenna channel electronically in the RF / mm-wave front-end. They can be used to realize a fixed beam to any direction or scan the beam rapidly in azimuth or elevation eliminating the need for any mechanical steering. Spatial filtering is the other benefit of phased arrays [3].

The number of commercial applications in the mm-wave frequency range have increased in the last decade. Miniaturization of complete mm-wave system and high resolution due to shorter wavelengths are the advantages of this frequency band, ranging from $30 \mathrm{GHz}$ up to $300 \mathrm{GHz}$. Point-to-point and short range high data rate communication systems at $60 \mathrm{GHz}$ [4] and driver assistance radars at $77 \mathrm{GHz}$ are the most popular examples of the mm-wave commercial applications.

The market for driver assistance systems based on mm-wave radar sensor technology is gaining momentum [5]. Radar can measure radial distance and velocity of remote objects very precisely. The goal is to relieve the driver from the combination of monotonic tasks and splitsecond decisions within complex traffic scenarios to improve safety and comfort. Fig. 1.1 shows different types of driver assistance systems [6]. Due to high number of applications, radar manufacturers are looking for multi-mode radar solutions. Thanks to its ability for adjusting a field of view and a beam shape, phased arrays can be used for fulfilling different applications using the same hardware (multi-mode antenna).

$7 \mathrm{GHz}$ of unlicensed bandwidth around $60 \mathrm{GHz}$ enables opportunities for high data rate wireless communications [7]. This bandwidth can be used for very high speed WLAN connectivity for indoor applications, as shown in Fig. 1.2. Small wavelengths at this frequency range make a complete phased array solution with relatively large number of antennas for such applications realizable. The phased array solution enhances the flexibility of the system in addressing multiple devices at the same time.

Despite its high functional performance compared to fixed beam antennas, phased arrays are still a very costly and complex solution for the applications mentioned above. Even more challenges are encountered with an increase in the number of transceiver channels if an antenna with high directivity is desired, such as routing of RF and IF circuits, isolation of 


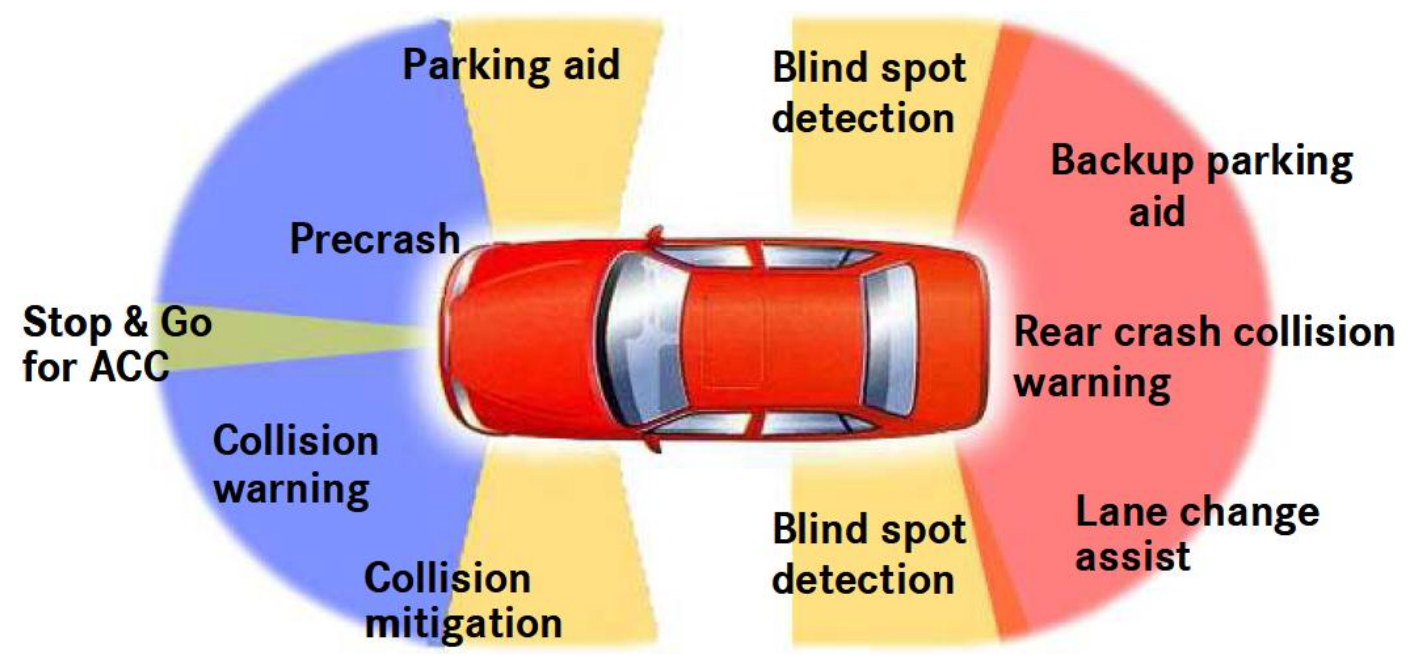

Figure 1.1.: Automotive radar applications.

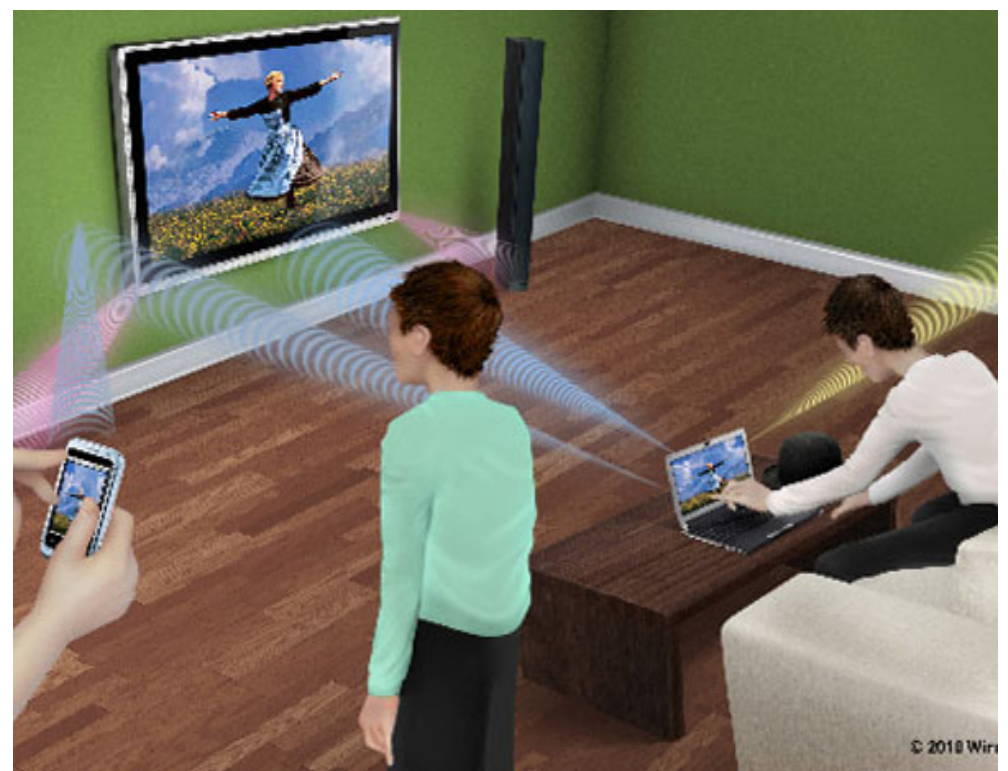

Figure 1.2.: High speed wireless data communication (Courtesy of Wireless Gigabit Alliance).

neighboring channels, and complex calibration of a whole system. 


\subsection{Conventional phased arrays}

As can be noted from its name, a 'phased array' is a directive antenna system, which employs multiple individual antenna sources. All antenna sources are spaced at a distance 'd' to each other. A phased array may be composed of a few units up to thousands of antenna elements. Different antenna elements may be used for the phased arrays. Most favorite ones are dipoles, open-ended waveguides, slotted waveguides and microstrip antennas. In order to control the shape and direction of the beam of the phased array, phase shifters and variable gain amplifiers are used. It enables a high speed electronic beam steering without any moving parts. This is the most conventional analog beamforming technique in use [8], [9]. Fig. 1.3 shows these parameters in a conventional phased array configuration.

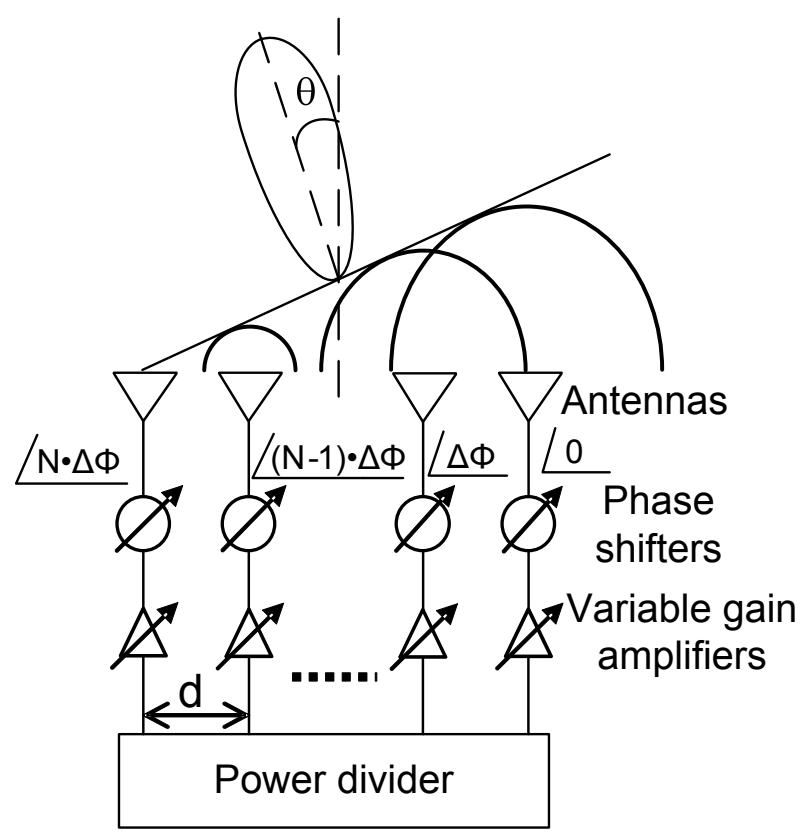

Figure 1.3.: A conventional phased array configuration.

By controlling the phase value $(\Delta \phi)$ of each phase shifter in each channel electronically, the direction of the main beam $(\theta)$ can be controlled. By providing variable gain $\left(a_{n}\right)$ in each channel using variable gain amplifiers, sidelobe levels can be suppressed, as shown in Fig. 1.5. Phase shifters are typically used in narrow band phased arrays whereas true-time delay elements are used for broadband phased arrays [10]. Analog phase shifters are used for continuous beam steering. Continuously steerable antennas are mainly preferred by adaptive arrays. On the other hand, digital phase shifters are employed for switched beam systems to create a number of fixed beams within the field of view.

The antenna pattern of a phased array can be obtained by the equation:

$$
R P(\theta)=A F(\theta) \cdot E F,
$$


where $R P$ refers to the total radiation pattern, $\theta$ to the beam direction, $A F$ to the array factor, and $E F$ to the element factor of a single antenna element. The array factor $A F$ for a linear patch array can be calculated as

$$
A F(\theta)=\sum_{n=1}^{N} a_{n} e^{j(n-1)(k d \sin (\theta)+\Delta \phi)},
$$

where $N$ refers to the number of antenna channels, $a_{n}$ to the magnitude of each signal, $d$ to the element spacing, and $\theta$ is the direction of main beam of the antenna and $\Delta \phi$ is the phase value of a phase shifter.

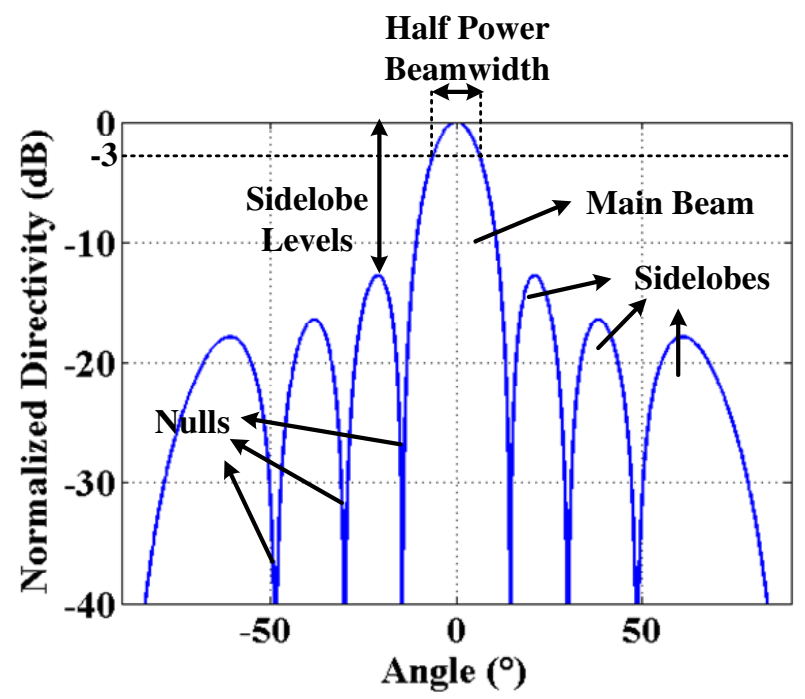

Figure 1.4.: A typical antenna pattern $(N=8, f=77 \mathrm{GHz}, \Delta \phi=0, d=0.5 \lambda)$.

A normalized plot of the antenna pattern of an array with 8 elements operating in the broadside mode is shown in Fig. 1.4. The main beam of radiation at $0^{\circ}$ can be seen, with nulls and sidelobes for angles of incidence moving towards $90^{\circ}$. The sidelobes are undesirable, as they result in interference by unwanted objects and wasted power. The effect of increasing the number of elements in the array is shown in Fig. 1.6. By increasing the number of elements, the width of the main beam is decreased while the number of sidelobes and nulls as well as the sidelobe levels increase. The field of view (FoV), half-power beamwidth (HPBW), sidelobe levels (SLL) and grating lobes are also affected by the element spacing, d. This is depicted in Fig. 1.7. The radiation pattern for a 4-element broadside array has been plotted for different element spacings. It can be seen that the width of the main beam is reduced, but the grating lobes appear within the field of view. By applying different phase shift values to the antenna elements, the main beam can be steered in the desired direction, as shown in Fig. 1.8.

Phased arrays can be classified into three different architectures, based on the location of the phase shifters: RF, IF and LO phase shifting [11], [12], [13], [14], [15]. For example, in 


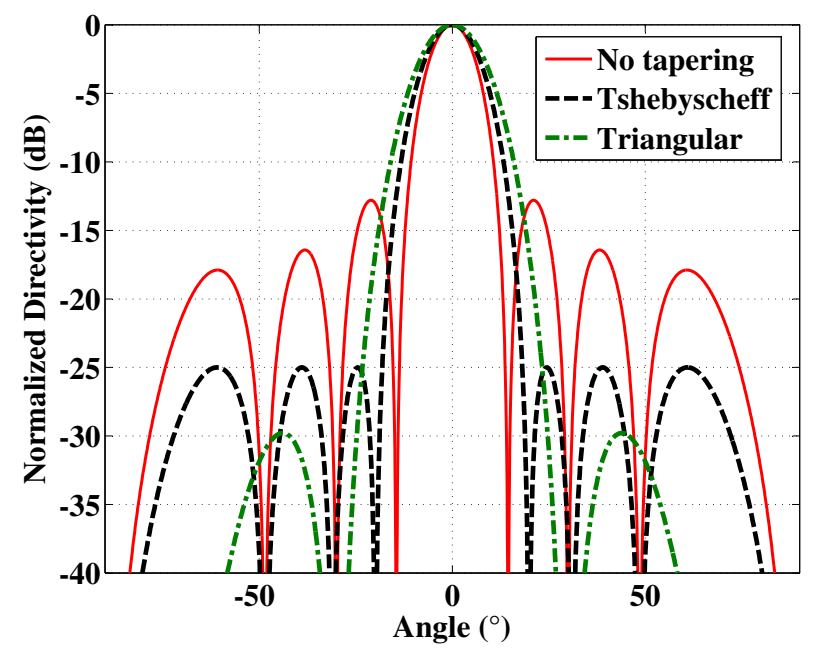

Figure 1.5.: Radiation patterns with different amplitude tapering methods ( $N=8, f=77 \mathrm{GHz}$, $\Delta \phi=0, d=0.5 \lambda)$.

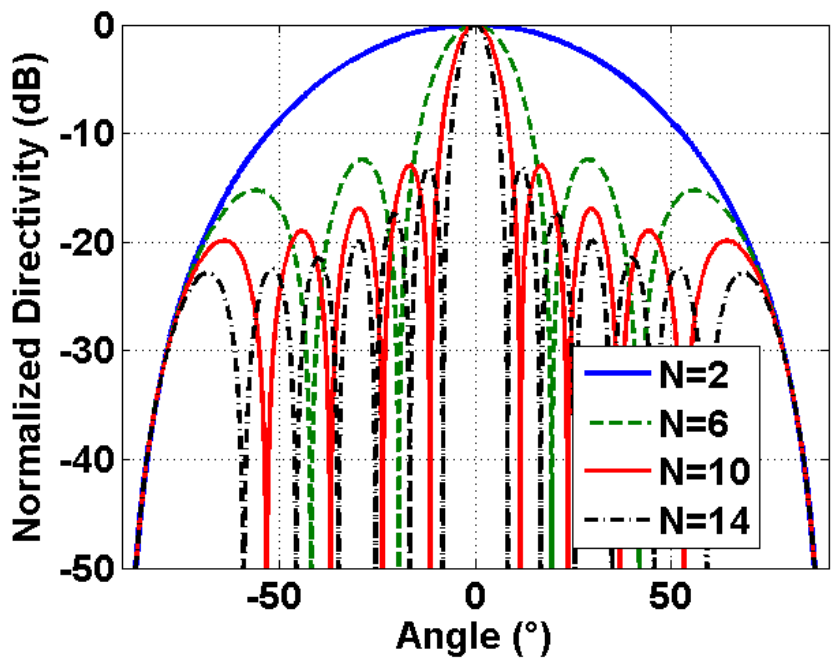

Figure 1.6.: Array factors (AF) for antenna arrays with $N=2, N=6, N=10$ and $N=14$ elements $(d=0.5 \lambda, f=77 \mathrm{GHz}, \Delta \phi=0)$.

the RF phase shifting architecture, the signals in the various channels are phase-shifted and combined in the RF domain. Among these phase shifting schemes, RF phase shifting is the most common and widely used phased array system because of its ability to suppress strong interference at the beginning of the RF path if it is used in the receiver side. Hence, sensitive components used in the RF, LO and IF paths are insulated from strong interference signals. RF phase shifting is usually preferred by military and satellite communication radars [16]. The combined signal is then down-converted to baseband signal using only a single mixer. Hence, the LO signal does not need to be distributed to each receiver path hence enabling a very compact architecture. 


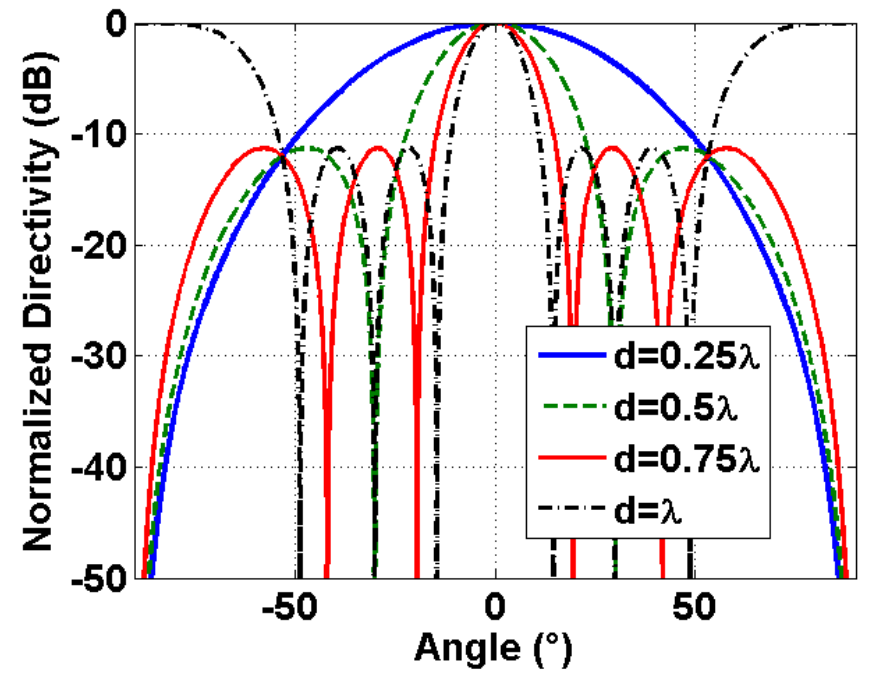

Figure 1.7.: Array factors (AF) for antenna arrays with $d=0.25 \lambda, d=0.5 \lambda, d=0.75 \lambda$ and $d=\lambda$ element spacings $(N=4, f=77 \mathrm{GHz}, \Delta \phi=0)$.

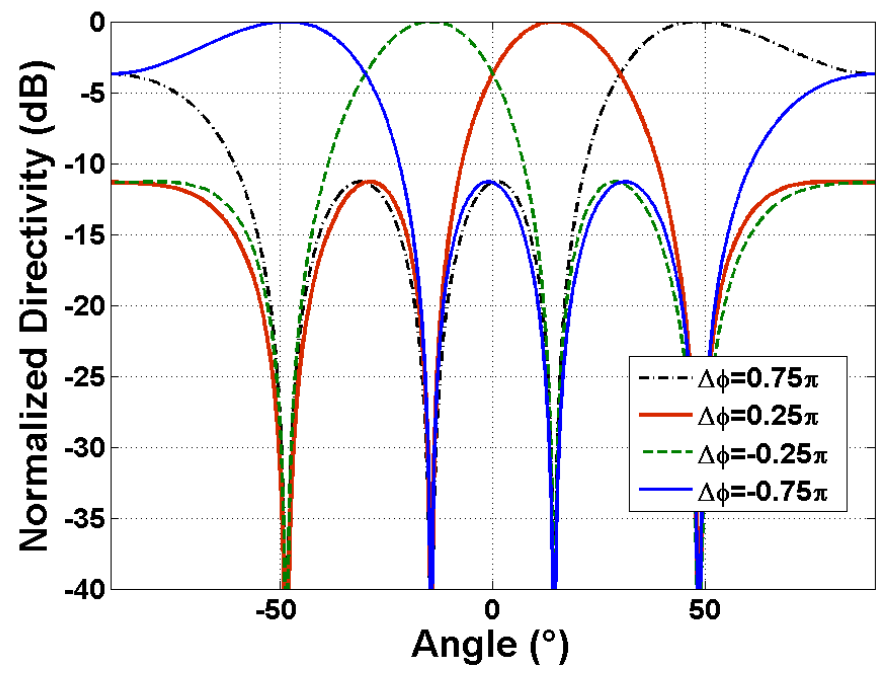

Figure 1.8.: Array factors (AF) for antenna arrays with $\Delta \phi=-0.75 \pi, \Delta \phi=-0.25 \pi, \Delta \phi=0.25 \pi$ and $\Delta \phi=0.75 \pi$ phase values $(N=4, f=77 \mathrm{GHz}, d=0.5 \lambda)$.

The use of a phased array increases the equivalent isotropic radiated power (EIRP). In the receiver phased array, beamforming improves the signal to noise ratio since the desired signals are integrated coherently. Also, due to the improved directionality of the beam, interference and multipath effects are minimized.

Until the last decade, the use of phased arrays in commercial applications was almost impossible due to the high cost of mm-wave phase shifters. The advent of low cost and high 
performance CMOS and SiGe technologies with transistor speeds higher than $100 \mathrm{GHz}$ and low-loss packaging solutions allow the realization of complex mm-wave systems for commercial applications [17], [18]. The silicon technology allows the integration of multiple channels of phase shifters and variable gain amplifiers on a single chip [11], [19]. However, there are still some challenges that need to be overcome, as will be discussed in the next section.

\subsection{Drawbacks of conventional phased arrays}

Despite the superior performance of the conventional phased arrays compared to fixed beam antennas, there are still some drawbacks that deter their use in commercial applications. Due to phase shifters and their control signals, phased arrays are still too costly to be implemented. To achieve a good antenna performance, a large number of array channels, and phase shifters, are needed. However, the increase in the number of channels brings extra problems and increases the level of the design complexity. These are; the number of interconnects to the active circuits, IF \& RF routings of the multiple channels, isolation between the channels and calibration of the whole system. Calibration takes a lot of time requiring complex software and hardware techniques. If an amplitude tapering is also employed in a phased array, variable-gain amplifiers must work in conjunction with the phase shifters. Amplitude and phase errors due to these RF components cause unwanted deviations in terms of sidelobe level, direction and beamwidth of the main beam.

RF designers are confronted with many challenging requirements during the design of a phased array. These are: Amplitude and phase mismatches between multiple channels, effect of packaging on an overall RF performance, connection of the array channels to the antennas, and channel-to-channel signal coupling which deteriorates the antenna array performance.

Even a slight change (a few microns) in the length of wire bonds and RF board traces for off-chip antennas causes an extra phase error at mm-wave frequencies. Therefore, it is very hard to make the fabrication process for a phased array employing multiple channels stable. In order to realize robust silicon-based single-chip antenna arrays for commercial communication and sensing applications, on-chip testing and calibration techniques are developed that measure the deterioration of array performance and correct the amplitudes and phases accordingly. In [20], a 16-channel silicon-based phased array is demonstrated. Even though phase and amplitude errors can be corrected via built-in self test systems, the transitions of the mm-wave signals to the 16 off-chip antennas around the chip and the stability of the fabrication process using many channels have remained unsolved. 


\subsection{A novel phased array approach}

Several new phased array techniques have been developed to overcome these problems, explained in the previous section. In [11], [21] and [22], multiple channels, including phase shifters, are integrated on a single chip to reduce cost. Still, complexity and calibration problems may be cumbersome for such MMIC designs. Also, the challenging interconnect issue between the transceiver front-end and multiple antenna elements still exists.

The work in [23] presents an electrically steerable microstrip antenna array composed of 5 elements at $5.8 \mathrm{GHz}$ ISM band. In this concept, phase shifters are added among patch elements of a bi-directional series-fed array. Although a wide range beam scanning is possible via this architecture, calibration and complexity barriers still exist due to the number of required phase shifters for large number of antenna elements ( $\mathrm{N}-1$ phase shifters for $\mathrm{N}$ radiating elements).

The work in [24] presents a beam steering approach at $2 \mathrm{GHz}$ by employing a single phase shifter to enable a low cost and less complex phased array system. In this approach, a grounded phase shifter is connected to the non-feeding end of a serially fed linear array, which provides a phase shifted reflected signal back to the array. The vector sum of the incident and phase shifted reflected signals at each radiating element in the array enables beam steering. However, in this concept, each antenna port still requires a separate variable gain amplifier to avoid amplitude variation during phase tuning of the phase shifter.

This thesis presents a new electronic beamforming approach that requires only a single phase shifter and two variable gain amplifiers (or attenuators) for a complete bi-directional seriesfed array, independent of the number of antenna elements, unlike a conventional phased array requiring one phase shifter for each channel. The main beam can be steered continuously to any direction within its maximum scan range with small variations in its gain. This approach eliminates the circuit complexity and cost, especially for the arrays with a large number of antenna elements. The approach is highly robust against phase and amplitude errors on the RF path. Channel mismatch and crosstalk between neighboring RF channels are eliminated via this technique, since only 2 channels are employed.

By using a hybrid ring coupler in a planar circuit or a magic-tee in a waveguide system, monopulse techniques can be applied to track the direction of the target accurately using the proposed antenna configuration [25], [26]. The information on the target direction is determined by the comparison of sum and difference antenna beams simultaneously. The angular position of a target can be extracted from only one pulse.

Due to its similar architecture, the proposed approach exhibits all the advantages that a traveling wave antenna possesses as well, such as beam steering with the change of the operating frequency. 
The presented concept can be especially useful in applications, where only a limited steering angle range (up to $\pm 20^{\circ}$ ) is required. For example, it can be used for electronic adjustment of the elevation angle of a radar sensor, where even a small misalignment in elevation can degrade the performance of the sensor, as shown in Fig. 1.9. A steering range of less $\pm 10^{\circ}$ can be sufficient in such cases.

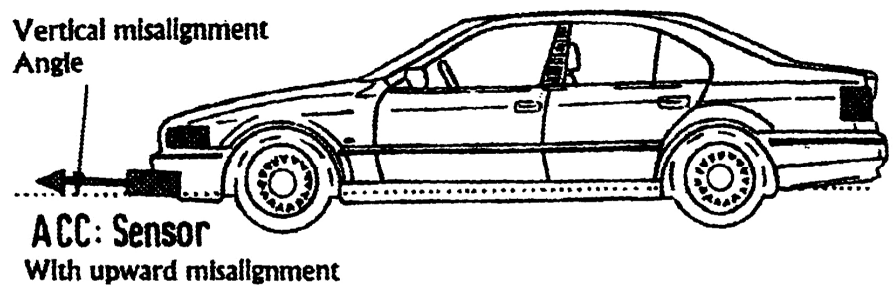

Figure 1.9.: Vertical misalignment of a radar sensor (Courtesy of BMW).

Another potential application for the presented concept is a beam steering in elevation for automotive radars. The proposed antenna system can distinguish the front target car from the bridge (or tunnel) above and cola can (or another metal object) on the ground in elevation, as shown in Fig. 1.10.

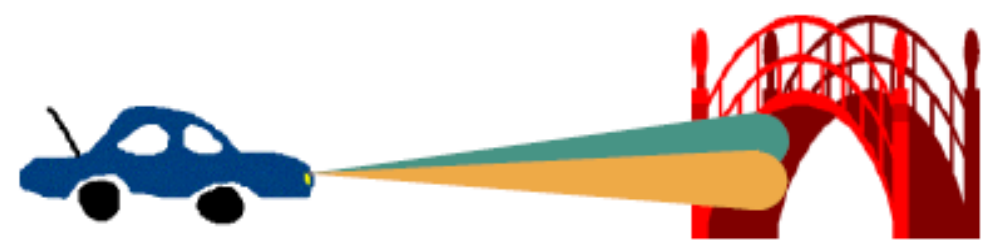

Figure 1.10.: Beam steering in elevation to detect a bridge.

\subsection{Dissertation overview}

This dissertation describes the design, realization and test of a novel mm-wave beam steering concept. 
In Chapter 2, the operating principle and the topology of the new approach are presented. The capabilities and limitations of the concept are investigated by simulating a linear patch array by means of a full-wave simulator at the operating frequency of $77 \mathrm{GHz}$. At the end of the chapter, its topology is compared with a conventional phased array.

Chapter 3 presents an analytical model, based on the closed form expressions, to design and optimize the dual-fed microstrip linear arrays. After demonstrating the validity of the model using full-wave simulations at different antenna structures, it is used to analyze the influence of the antenna geometry and operating frequency on the far field pattern of the antenna pattern.

In Chapter 4, mm-wave components, required for realization of two different antenna measurement setups, are analyzed and presented. A $77 \mathrm{GHz}$ MMIC transceiver that incorporates an integrated I-Q vector modulator is characterized for use as a phase shifter and a variable attenuator in the test setup. Next, a low-loss and broadband waveguide to microstrip line transition is designed and realized at $76-81 \mathrm{GHz}$ frequency range. Finally, a mm-wave rat-race balun is characterized to convert differential signals to single ended ones.

Chapter 5 demonstrates two different measurement test setups to verify the new concept and reports the results of measurement and simulation results.

Chapter 6 summarizes the work and provides recommendations for a further research. 


\section{A Novel Phased Array Concept}

In this chapter, a novel phased array approach is presented. In order to explain the working principle, array theory of the proposed concept is first introduced in Section 2.1. The capabilities of the new concept are analyzed in Section 2.2 by simulating a novel phased array concept with a planar array antenna.

\subsection{Array theory}

As shown in Fig. 2.1, the incident wave, feeding one of the two input ports, is distributed consecutively to each individual element, where a certain fraction of the incident power radiates, while the rest continues to propagate through feed lines to the next element until the remaining signal is terminated at the opposite port. This resembles the well known traveling wave antenna concept [27]. Using appropriate feed line lengths among the antenna elements, a squinted antenna beam can be generated. In this case, beam steering can only be performed via change of operating frequency [28].

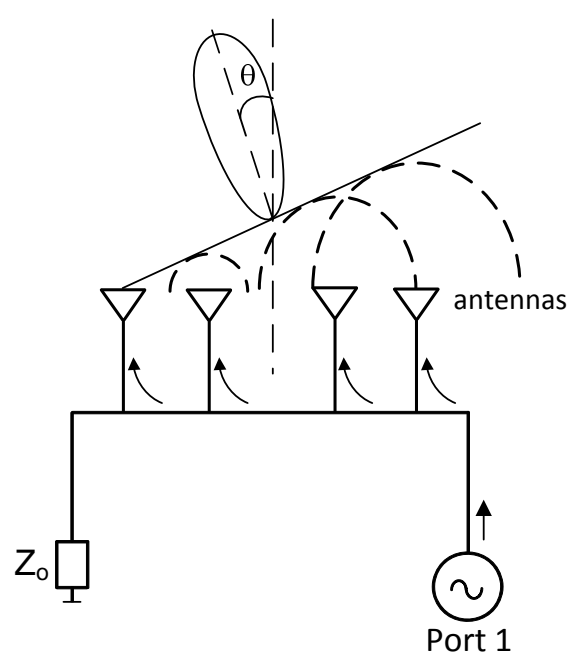

Figure 2.1.: A traveling wave antenna and resulting beam / wave front.

The proposed design employs a series-fed bi-directional array, realized using planar patch antenna elements, as shown in Fig. 2.3.

By feeding an additional signal at the second input port simultaneously, a superposition of two radiation patterns can be generated, as depicted in Fig. 2.2. The overall pattern can be 


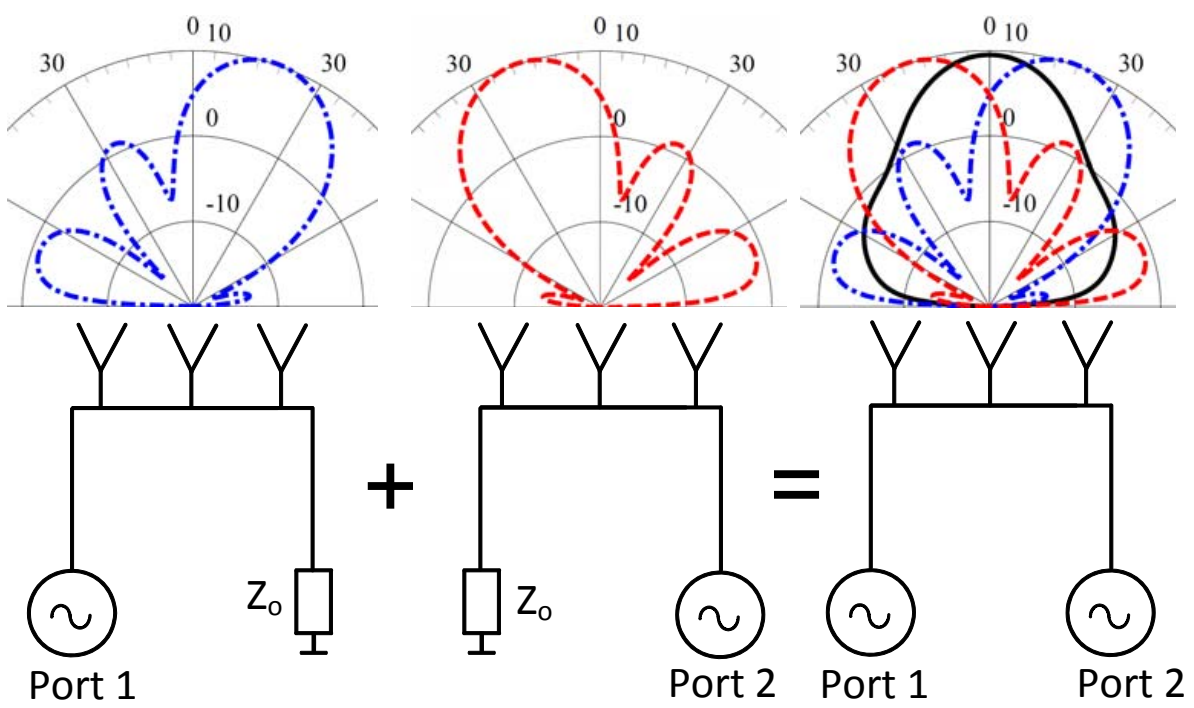

(a)

(b)

(c)

Figure 2.2.: Working principle of the dual-fed phased array, feeding a linear array at (a) only Port 1, (b) only Port 2, (c) Ports $1 \& 2$.

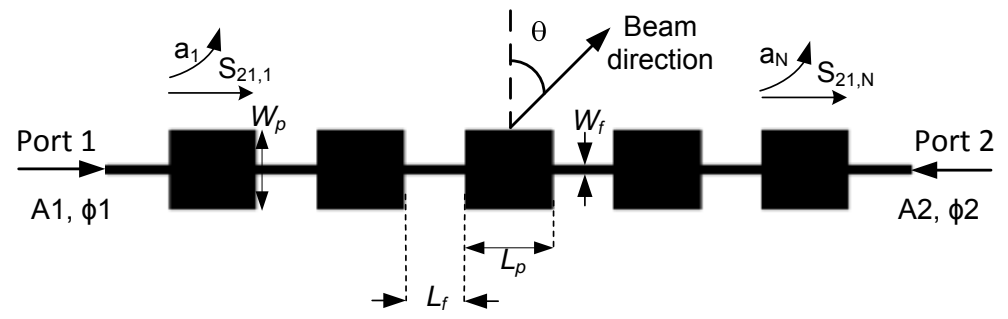

Figure 2.3.: Linear array using series-fed patch antenna elements.

obtained using the following equation:

$$
R P(\theta)=\left(A_{1} \cdot A F(\theta)+A_{2} \cdot A F(-\theta) \cdot e^{j \phi_{\mathrm{ps}}}\right) \cdot E F,
$$

where $R P$ refers to the total radiation pattern, $\theta$ to the beam direction, $A_{1}$ and $A_{2}$ to the amplitudes, and $\phi_{1}$ and $\phi_{2}$ to the phases of the excitation signals at the input ports. The amplitude is normalized to $A_{1}^{2}+A_{2}^{2}=1$ and the phase difference is $\phi_{\mathrm{ps}}=\phi_{2}-\phi_{1}$. $\phi_{\mathrm{ps}}$ refers to the phase difference between the excitation signals, $A F$ to the array factor when only port 1 or port 2 is fed, and $E F$ to the element factor of a single antenna element. The array factor $A F$ for a linear patch array can be calculated as

$$
A F(\theta)=\sum_{n=1}^{N} a_{n} e^{-j 2 \pi(n-1)\left(\frac{\left(L_{p}+L_{f}\right) \sin \theta}{\lambda_{0}}+\frac{L_{f}}{\lambda_{g}}\right)}
$$

with the antenna dimensions $L_{p}, W_{f}$, and the feed line length $L_{f}$ between the individual elements, as shown in Fig. 2.3. $\lambda_{0}$ is the freespace wavelength, $\lambda_{g}$ the guide wavelength of 
the feed line. The amplitude distribution $a_{n}$ between the antenna elements can be calculated as

$$
a_{n}=\sqrt{1-\left|S_{21, n}\right|^{2}} \cdot \prod_{i=1}^{n-1} S_{21, i}
$$

where $S_{21, i}$ is the transfer function of a single antenna element, and $N$ the number of antenna elements. By adjusting the $S_{21, n}$ coefficients, the antenna can be designed to have a desired radiation pattern and still retain a good antenna efficiency, i.e. only little power reaches the opposite end of the antenna.

To achieve the maximum scan range of $\theta= \pm \theta_{m}$ without amplitude deviation, the antenna factor $A F$ must adhere to:

$$
A F(0)=\frac{1}{\sqrt{2}} \cdot A F\left(\theta_{m}\right)
$$

This allows a continuous steering of the antenna beam maximum from $\theta=\theta_{m}$ with $A_{1}=$ $1, A_{2}=0$ over $\theta=0$ using $A_{1}=A_{2}=0.707, \phi_{\mathrm{ps}}=180^{\circ}$ to $\theta=-\theta_{m}$ at $A_{1}=0, A_{2}=1$. Larger scan ranges can be achieved if some amplitude variation is accepted.

The sum $(\Sigma)$ and difference $(\Delta)$ radiation patterns can be obtained if equal amplitude values $\left(\Delta A_{1}=\Delta A_{2}\right)$ and $180^{\circ} / 0^{\circ}$ phase difference $\left(\phi_{p s}\right)$ are supplied respectively.

\subsection{Dual-fed phased array demonstrator employing a planar array}

\subsubsection{Planar array}

Planar arrays are attractive for use in mm-wave applications due to their low cost, low profile and simple integration with the rest of the circuits [29]. A series-fed planar array is made up of rectangular patch elements interconnected by high impedance feed lines. These feed lines introduce a progressive phase shift that makes a beam squint in the E-plane. If all patches have equal width, the excitation decays exponentially along the array. The amount of radiation from a patch element can be controlled by tapering the appropriate patch width. With this method, the sidelobe levels can be suppressed. The width of the patch should be chosen carefully in order to suppress higher order modes. By adjusting the element spacing, the beam can be tilted away from the broadside direction. For a broadside radiation pattern, the element spacing between subsequent patches and the length of patches should be $\lambda_{g} / 2$, as shown in Fig. 2.4. If the element spacing is less than $\lambda_{g} / 2$, the beam, depicted in Fig. 2.5(b), squints towards the source side, while a spacing larger than $\lambda_{g} / 2$ results in a squint towards the load side, as shown in Fig. 2.5(a). The direction of the main beam also depends on the location of the port from which the signal is fed. 


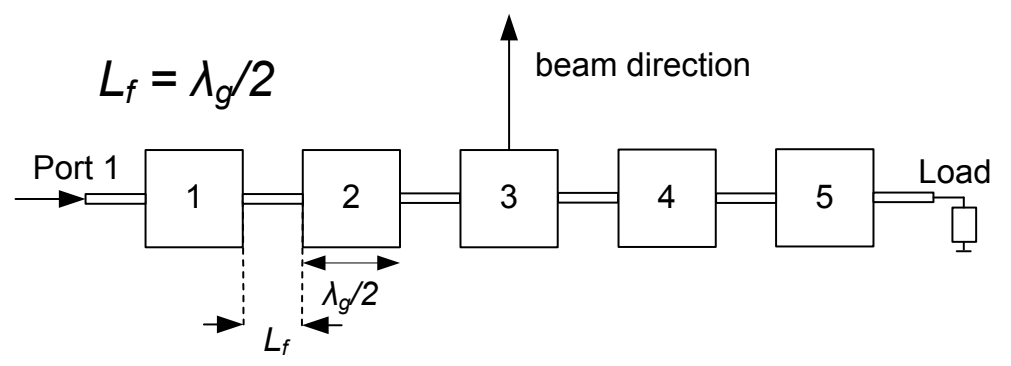

Figure 2.4.: Direction of the main beam for $L_{f}=\lambda_{g} / 2\left(L_{p}=\lambda_{g} / 2\right)$.

(a) Direction of the beam for $L_{f}>\lambda_{g} / 2$.

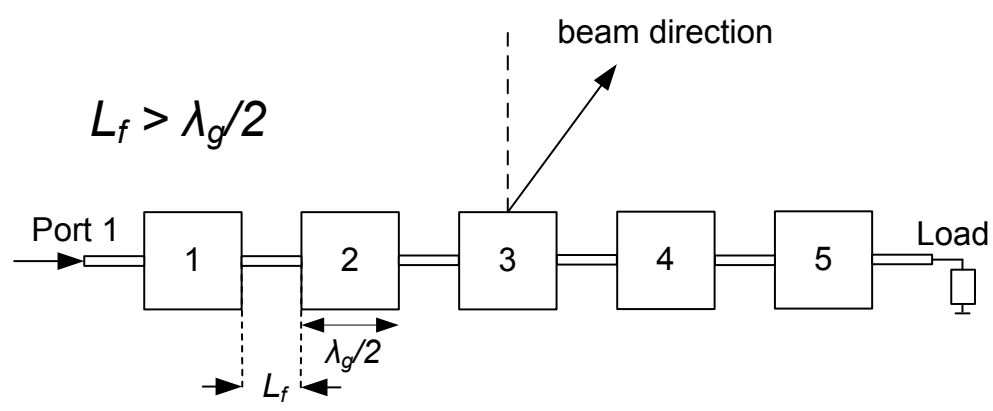

(b) Direction of the beam for $L_{f}<\lambda_{g} / 2$.

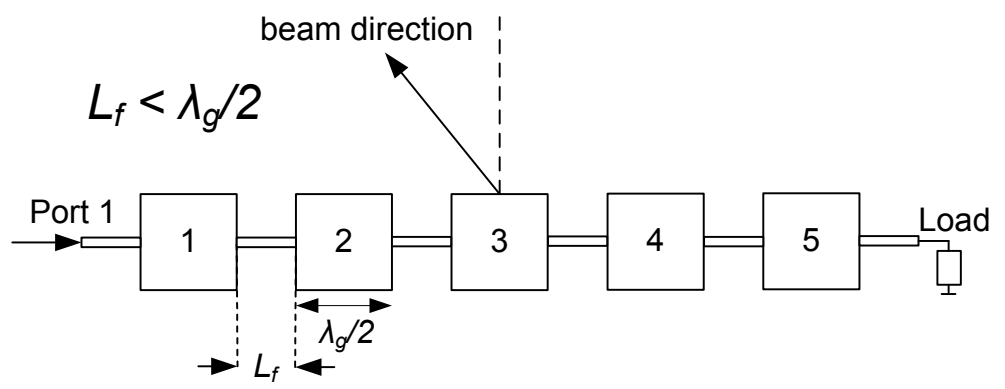

Figure 2.5.: Direction of the main beam based on the feed line length $\left(L_{f}\right)$ between patches for $L_{p}=\lambda_{g} / 2$.

\subsubsection{Simulation and Optimization}

To investigate the beam steering capabilities, a microstrip linear array was designed and simulated using a $0.127 \mathrm{~mm}$ thick RO3003 substrate $\left(\epsilon_{r}=3\right)$ [30]. As shown in Table A.2, all patch elements have the same size in the same array. The length of each patch element $\left(L_{p}\right)$ is taken to be slightly shorter than $\lambda_{g} / 2$ at the desired operating frequency because of the fringing effects at the edges of the radiating patches [27]. The width of each patch $\left(W_{p}\right)$ determines the coupled power. However, higher order modes appear if the width is too large. Each patch is connected to the high impedance microstrip feed lines from both sides. For different array configurations, only the number of patches $(N)$ and the length of feed lines $\left(L_{f}\right)$ are varied. 


\section{Operating frequency}

By changing the operating frequency of a signal, beam steering can be achieved since change of the operating frequency has direct effect on the change of phase difference between subsequent elements in an array. A full-wave simulation of a linear array is carried out employing 5 patch elements $(N=5)$ and $1.5 \mathrm{~mm}$ long feed lines $\left(L_{f}=1.5 \mathrm{~mm}\right)$ in the array. Other parameters used for the simulations are listed in Table A.1 and Table A.2. Fig. 2.6 shows the beam steering capability of the dual-fed antenna if the operating frequency is varied between 75 and $81 \mathrm{GHz}$. The scan angle stays between $5^{\circ}$ and $14^{\circ}$ at the frequency band of interest. At the scan angle of $5^{\circ}$, the HPBW is $18^{\circ}$, whereas at the scan angle of $14^{\circ}$, the HPBW is $16^{\circ}$, which is slightly narrower. If the port that supplies the signal is moved to the other port, the beam pattern shifts to the other half of the field of view.

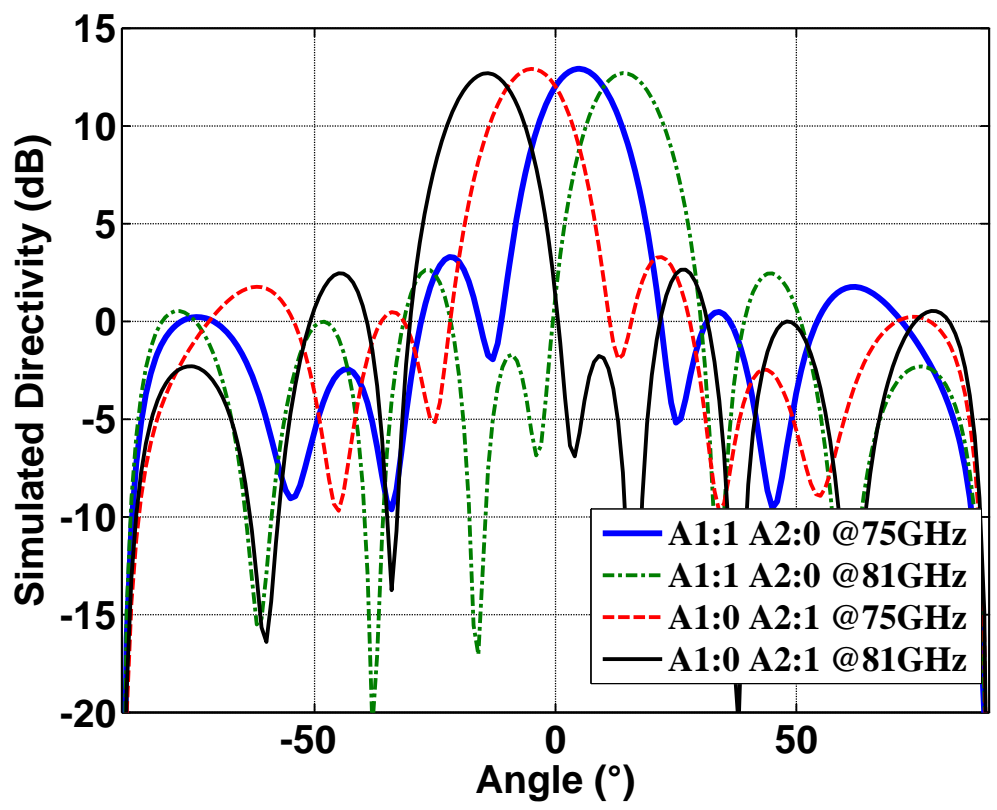

Figure 2.6.: Beam steering by change of the operating frequency from 75 to $81 \mathrm{GHz}$.

\section{Amplitude and phase of the supply signal}

Using the optimized parameters listed in Table A.2, the linear array shown in Fig. A.2 is simulated at the operating frequency of $77 \mathrm{GHz}$ by choosing the lengths of the feed lines $\left(L_{f}\right)$ to be $0.85 \mathrm{~mm}$ and number of patch elements to be $N=5$. Fig. 2.7 shows the simulated beam steering capability of this array configuration. A maximum scan range of $28^{\circ}$ can be achieved by adjusting the amplitude and the phase values of the supplied signals, which are shown in the same figure. All side lobe levels are less than $-10 \mathrm{~dB}$. 
Table 2.1.: Optimized physical parameters of rectangular patches used for a 77-GHz linear array (Fig. A.2)

\begin{tabular}{l|l} 
Physical parameter & Value \\
\hline Length of a patch $\left(L_{p}\right)$ & $1.09 \mathrm{~mm}$ \\
Width of a feed line $\left(W_{f}\right)$ & $0.1 \mathrm{~mm}$ \\
Width of a patch $\left(W_{p}\right)$ & $1.016 \mathrm{~mm}$
\end{tabular}

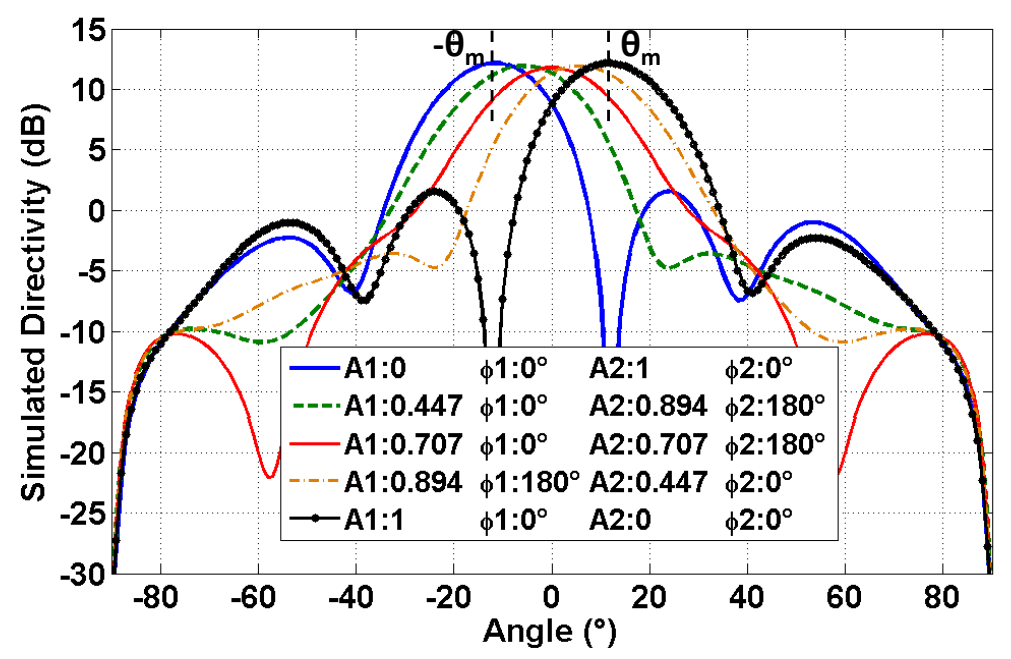

Figure 2.7.: Beam steering using a phase shifter and an attenuator $\left(L_{f}=0.85 \mathrm{~mm}, N=5\right)$ (Simulation).

\section{Generation of the sum and difference beam}

Fig. 2.8 shows the sum $(\Sigma)$ and difference $(\Delta)$ antenna beams of a linear array configuration consisting of 5 microstrip patches $(N)$ with $1.5 \mathrm{~mm}$ long feed lines $\left(L_{f}\right)$. The sum pattern can be obtained with a $180^{\circ}$ phase difference of the supplied signals. This pattern has the HPBW of $18^{\circ}$ with a directivity of $12.5 \mathrm{~dB}$. The first sidelobe levels are less than $-22 \mathrm{~dB}$. The difference pattern is obtained via a $0^{\circ}$ phase difference. The broadside null radiating beam has a dynamic range higher than $40 \mathrm{~dB}$. The main beams look to the directions of $\pm 12^{\circ}$ with the HPBW of $15^{\circ}$. The maximum directivity of the difference pattern is almost $3 \mathrm{~dB}$ less than the maximum directivity of the sum pattern.

\section{Effects of amplitude and phase errors}

Amplitude and phase errors induced by the mm-wave components on the RF path lead to increased sidelobe levels, deviation in the desired direction of the main beam and reduction in directivity [31]. Thanks to its technique, this concept is highly robust against these errors. Fig. 2.9 shows effects of amplitude and phase errors between both channels on an overall radiation pattern. For validation, the sum pattern, shown in Fig. 2.8, is used. Fig. 2.9(b) shows 


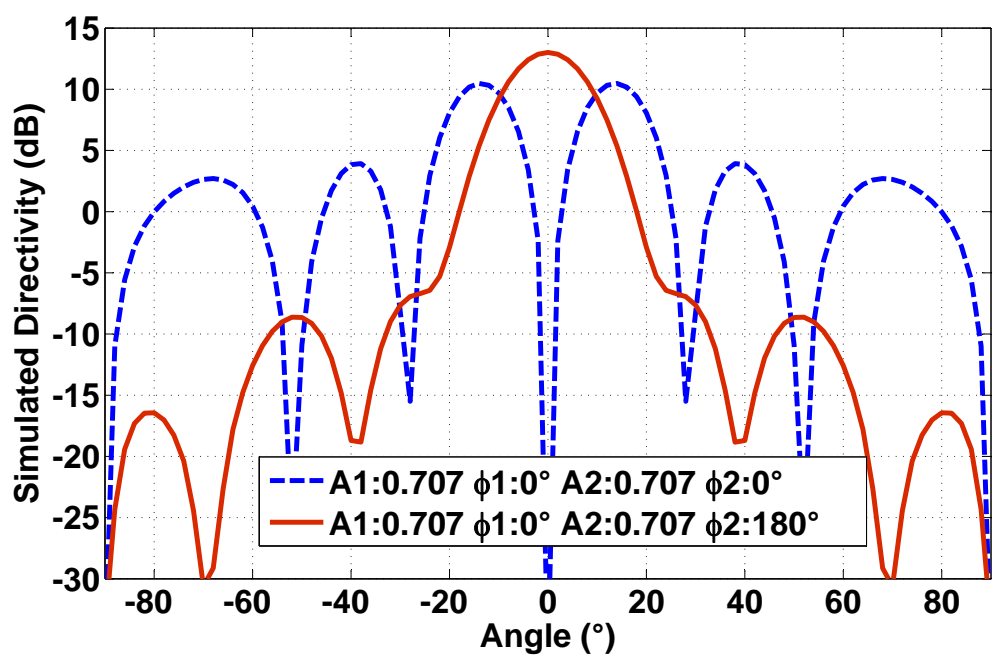

Figure 2.8.: Sum $(\Sigma)$ / difference $(\Delta)$ antenna patterns which are the solid and dashed curves respectively $\left(L_{f}=1.5 \mathrm{~mm}, N=5\right)$ (Simulation).

that a phase error up to $\pm 30^{\circ}$ leads to almost no change in the beam direction and HPBW of the main lobe. The ability of steering one or more null beams of the radiation pattern towards some interferences without affecting the performance of the main beam is especially important for wireless communication systems [32], [33]. By the variation of the phase difference between the two ports, the null beams can be directed in the desired directions, as shown in Fig. 2.9(b). Fig. 2.9(a) shows different simulated patterns including an amplitude error of up to $\pm 1.94 \mathrm{~dB}$ ( $\pm 20 \%$ deviation from the original value). This error causes almost no change in the beamwidth and only $\mathrm{a} \pm 1^{\circ}$ shift at the direction of the main beam, whereas the directions of the sidelobes remain unchanged.

\section{Effect of number of elements}

Fig. 2.10(a) and Fig. 2.10(b) show the effect of the number of elements on the scan range by keeping the length between patch elements $L_{f}$ same. Only, $L_{f}=0.8 \mathrm{~mm}$ ensures a continuous beam scan with $N=4$, whereas an array employing 10 elements cannot provide a continuous beam scan anymore (Only discrete beam scan is possible).

Table 2.2 shows a comparison of the simulation parameters for three arrays with a different number of antenna elements $N$. The length of the feed lines is optimized for a maximum continuous beam steering within the given scan range at $77 \mathrm{GHz}$. An increase in the number of elements decreases the maximum beam scan range, whereas the HPBW becomes narrower and the directivity increases. A maximum scan range of $32^{\circ}$ is possible using a 4-element linear array with $0.8 \mathrm{~mm}$ long feed lines. The increase in the number of radiating elements leads to higher total efficiency, which is defined as the ratio of the total radiated power to the total input power supplied to the linear array, including mismatches. 
(a) Amplitude errors

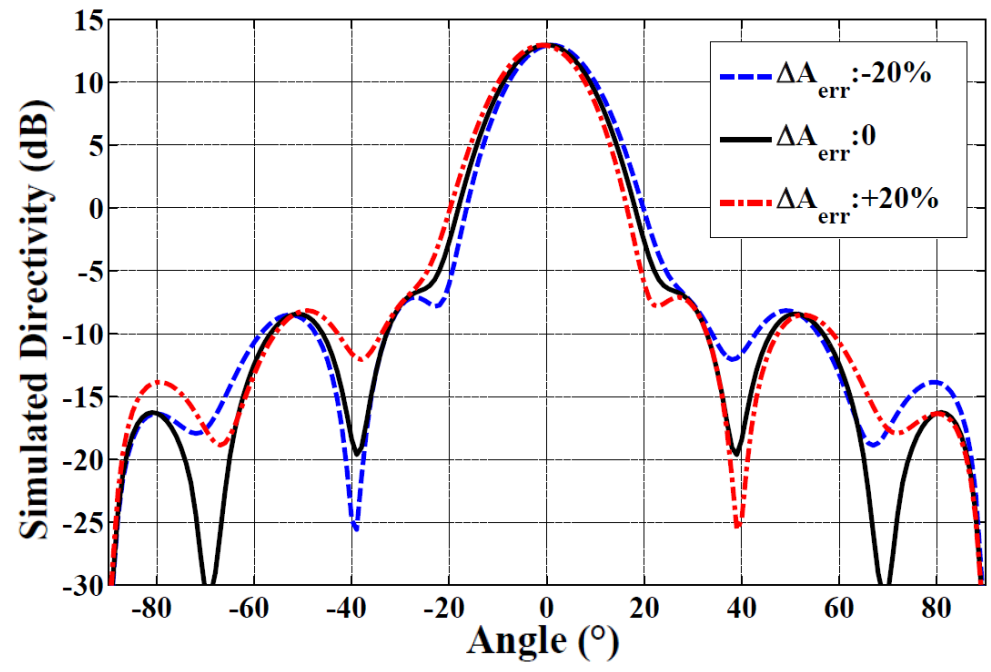

(b) Phase errors

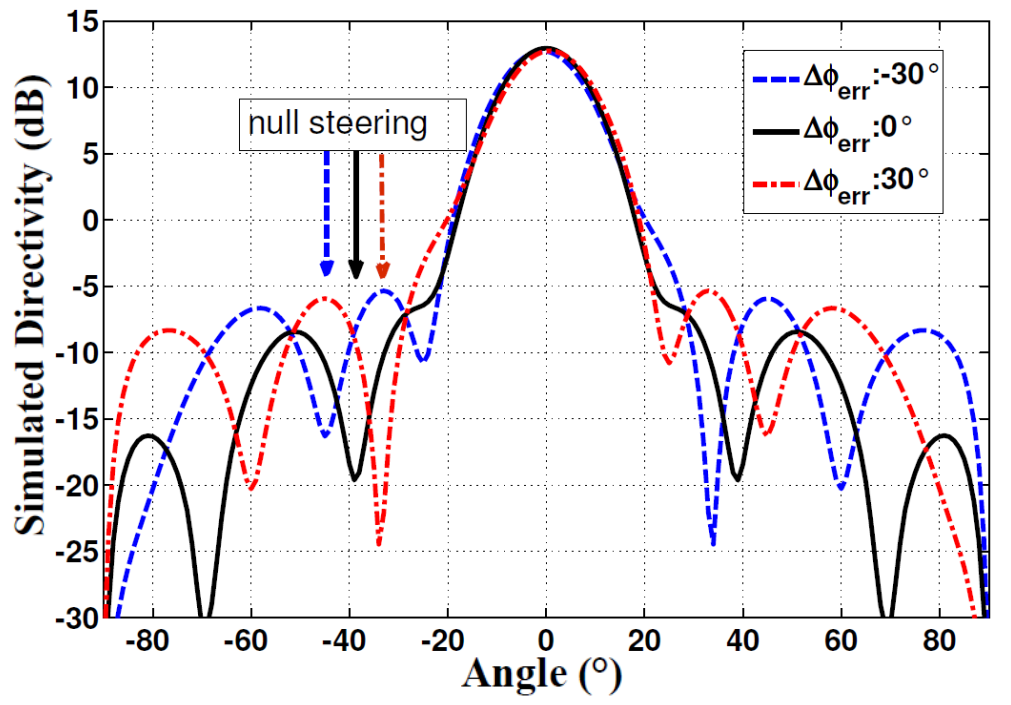

Figure 2.9.: Effect of amplitude and phase errors on a sum antenna pattern $\left(L_{f}=1.5 \mathrm{~mm}\right.$, $N=5)$ (Simulation).

\section{Effect of feed line length}

Table 2.3 shows the effect of varying the feed line lengths $\left(L_{f}\right)$ on the antenna parameters. The rectangular microstrip patches can be represented as two radiating slots with a spacing of $L_{p}=\lambda_{g} / 2$. If feed line lengths between radiating patches are also chosen as half of the guide wavelengths $\left(L_{f}=\lambda_{g} / 2=1.23 \mathrm{~mm}\right)$, then all radiating slots have the same phase value, as shown in Fig. 2.11. Therefore, a beam is always directed to the broadside direction, independent of the amplitude and phase values of the input signals fed into the array. The 
(a) Continuous beam scan up to $\theta_{m}= \pm 16^{\circ}$ using $L_{f}=0.8 \mathrm{~mm}$ and $N=4$ (Simulation).

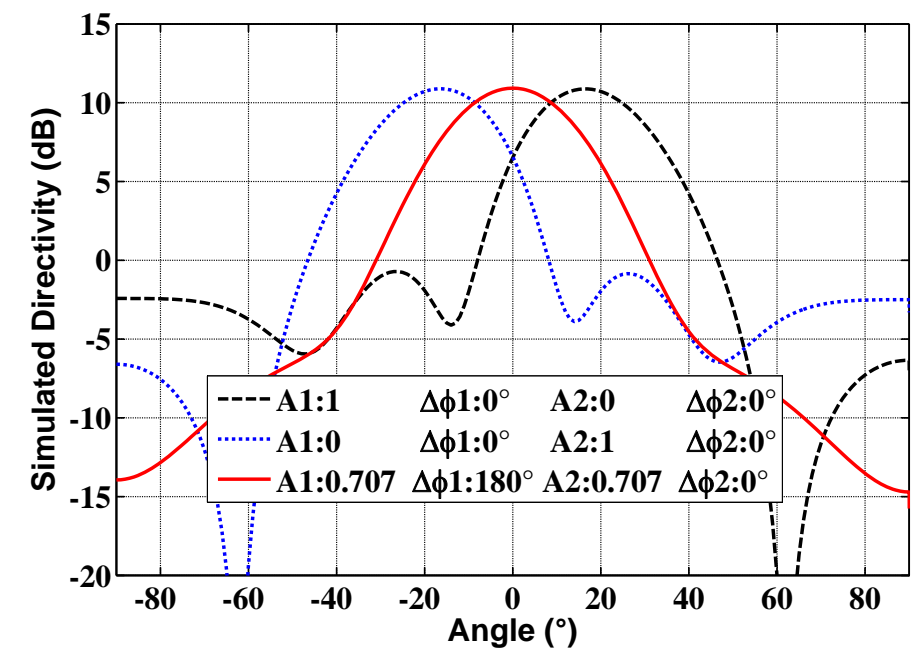

(b) Discrete beam scan up to $\theta_{m}= \pm 16^{\circ}$ using $L_{f}=0.8 \mathrm{~mm}$ and $N=10$ (Simulation).

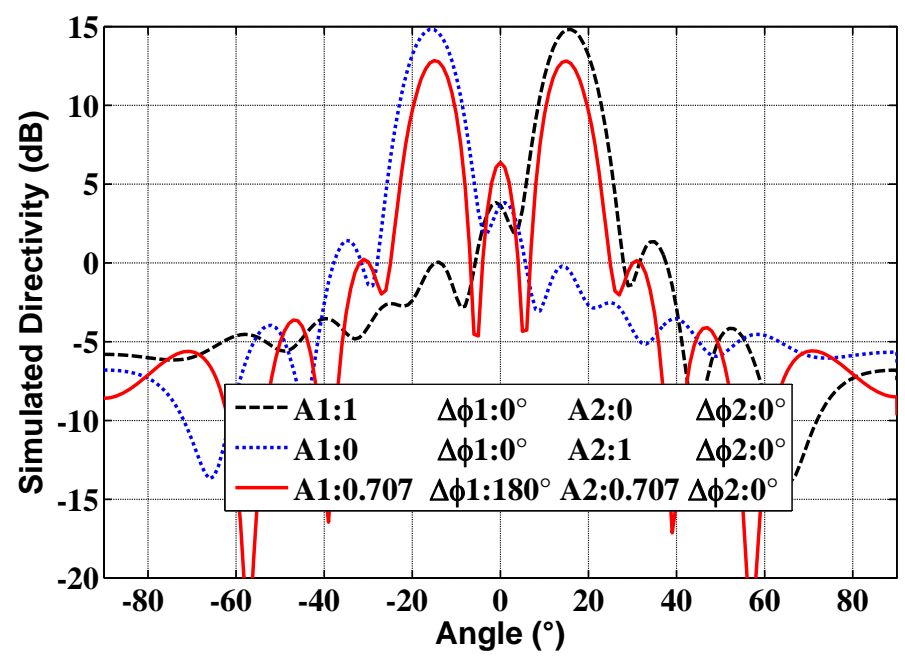

Figure 2.10.: Comparison of continuous and discrete beam scans employing same feed line lengths $\left(L_{f}\right)$ and different number of antenna elements $(N)$.

maximum scan range increases if $L_{f}$ is shorter or longer than $\lambda_{g} / 2$. According to the simulation results, grating lobes appear within the field of view if $L_{f}$ is chosen very large. The total efficiency is minimum if $L_{f}=\lambda_{g} / 2$ although this leads to the highest directivity. The longer the feed lines are, the narrower the HPBW is. There are slight changes in terms of maximum sidelobe levels, as shown in Table 2.3. 
Table 2.2.: Change of number of patch elements $(N)$ at $77 \mathrm{GHz}$.

\begin{tabular}{l|c|c|c}
\hline Simulated & \multicolumn{3}{|c}{ Number of patch elements } \\
\cline { 2 - 4 } Parameters & $\mathbf{4}$ & $\mathbf{5}$ & $\mathbf{1 0}$ \\
\hline Length of feed lines $\left(L_{f}\right)$ & $0.8 \mathrm{~mm}$ & $0.85 \mathrm{~mm}$ & $1.35 \mathrm{~mm}$ \\
\hline $\begin{array}{l}\text { Max. continuous beam scan } \\
\text { range }\end{array}$ & $32^{\circ}$ & $28^{\circ}$ & $12^{\circ}$ \\
\hline Max. sidelobe levels & $-11.6 \mathrm{~dB}$ & $-12.5 \mathrm{~dB}$ & $-11.4 \mathrm{~dB}$ \\
\hline 3-dB beamwidth (HPBW) & $29^{\circ}$ & $22^{\circ}$ & $8^{\circ}$ \\
\hline Directivity & $11 \mathrm{~dB}$ & $12 \mathrm{~dB}$ & $16.2 \mathrm{~dB}$ \\
\hline $\begin{array}{l}\text { Total efficiency including } \\
\text { mismatch }\end{array}$ & $52 \%$ & $58 \%$ & $63 \%$ \\
\hline
\end{tabular}

Table 2.3.: Change of length of feed lines $\left(L_{f}\right)$ at $77 \mathrm{GHz}$.

\begin{tabular}{l|c|c|c}
\hline Feed line length & $\mathbf{0 . 8 5} \mathbf{~ m m}$ & $\mathbf{1 . 2 3} \mathbf{~ m m}$ & $\mathbf{1 . 5} \mathbf{~ m m}$ \\
\hline Number of patch elements & 5 & 5 & 5 \\
\hline $\begin{array}{l}\text { Max. continuous beam scan } \\
\text { range }\end{array}$ & $28^{\circ}$ & $0^{\circ}$ & $16^{\circ}$ \\
\hline Max. sidelobe level & $-12.5 \mathrm{~dB}$ & $-12 \mathrm{~dB}$ & $-11.7 \mathrm{~dB}$ \\
\hline 3-dB beamwidth (HPBW) & $22^{\circ}$ & $17.2^{\circ}$ & $15.5^{\circ}$ \\
\hline Directivity & $12 \mathrm{~dB}$ & $13.6 \mathrm{~dB}$ & $12.9 \mathrm{~dB}$ \\
\hline $\begin{array}{l}\text { Total efficiency including } \\
\text { mismatch }\end{array}$ & $58 \%$ & $39 \%$ & $51 \%$ \\
\hline
\end{tabular}

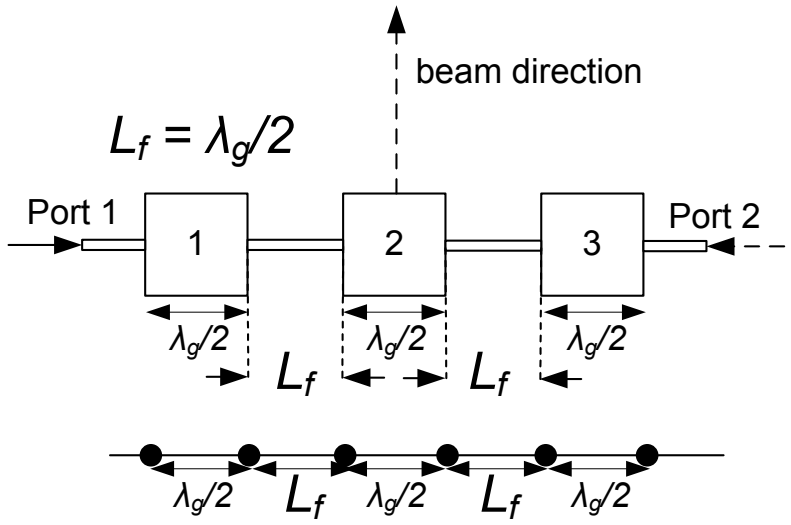

Figure 2.11.: Equivalent model of the linear patch array.

\section{Antenna candidates for novel phased array concept}

Different antennas can be used with this approach. A dual-fed linear array is the backbone of this approach. The linear array configuration can be a patch array (Fig. 2.12) or a waveguide slot array (Fig. 2.13). In this dissertation, an antenna setup shown in Fig. 2.12 will be used 
for analysis and characterization. If a narrow beam is desired in E- and H-planes, a planar array can be employed, as shown in Fig. 2.14.

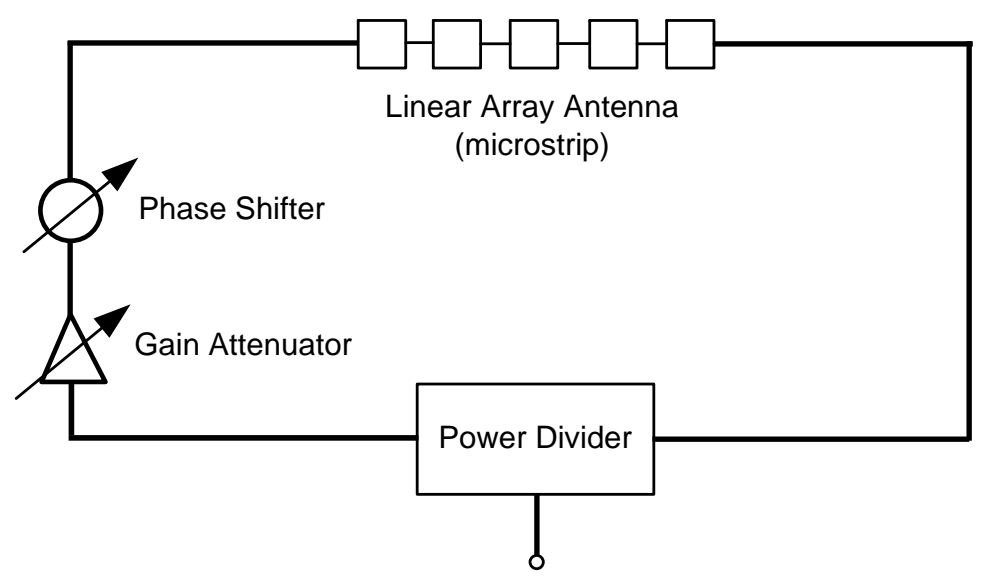

Figure 2.12.: An example for a dual-fed microstrip array antenna.

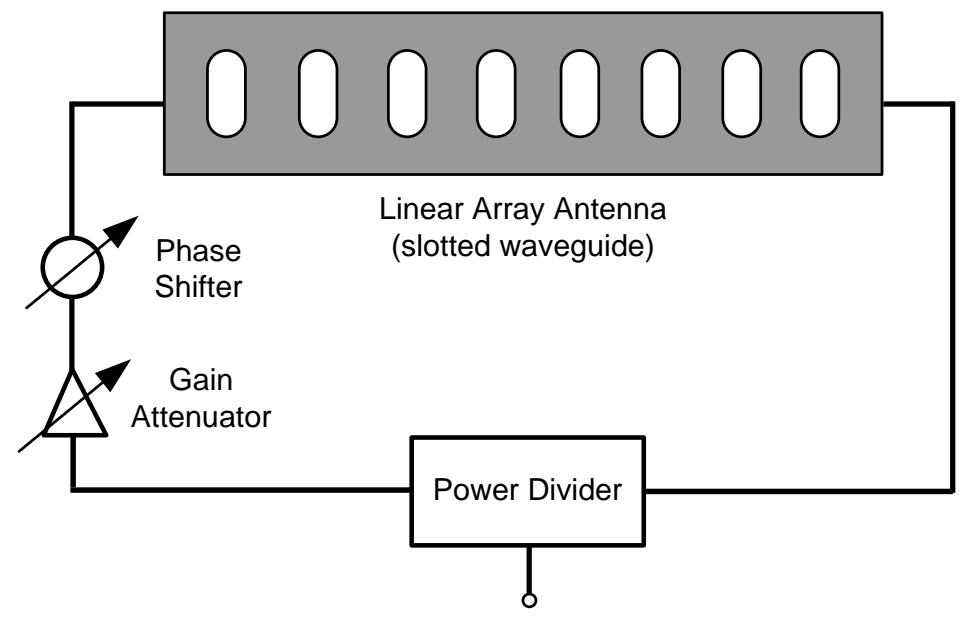

Figure 2.13.: An example for a dual-fed slotted waveguide array antenna. 


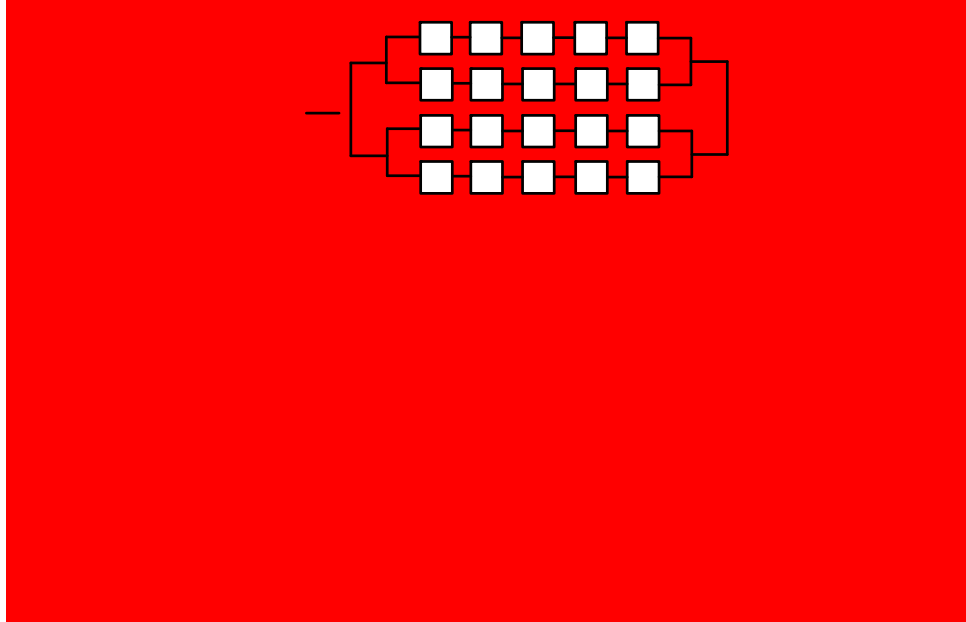

Figure 2.14.: An example for a dual-fed microstrip planar array antenna. A narrow beam can be obtained in both azimuth and elevation. 


\section{Analytical Antenna Model}

In this chapter, an analytical model is derived in MATLAB that is used for designing and optimizing the dual-fed microstrip linear arrays. This model allows fast computation and optimization without a long optimization time and huge computational memory. This model has been optimized by assuming that the antenna structure is printed on a dielectric substrate and operating within the desired frequency range of $75 \mathrm{GHz}$ to $85 \mathrm{GHz}$.

In Chapter 3.1, the main advantages and limitations of the proposed model are provided. Chapter 3.2 shows the development procedure and the validity of the model using numerical simulations. Finally in Chapter 3.3, the design parameters of a microstrip linear array antenna are investigated to analyze effects of each parameter on the overall antenna pattern for a dualfed linear array.

\subsection{Introduction}

The analysis of microstrip lines can be classified into 3 categories; quasi-static only, quasistatic including dispersion effects and full-wave analysis [34]. In the quasi-static analysis, the fundamental propagation mode of a microstrip line is assumed as a pure TEM mode. This method can be used for microstrip line structures for up to a few $\mathrm{GHz}$. When the dispersion effects are included, the effective dielectric permittivity and the characteristic impedance become frequency dependent. These parameters vary in a non-linear manner as the operating frequency is increased. This analysis is useful for designs at the mm-wave frequency range. In full-wave analysis, S-parameters are computed considering all physical effects that cannot be explained with other methods accurately. Although the last analysis method offers the most accurate results, it is very time-consuming during the development phase.

Since a serially fed traveling wave linear array is the starting point of the approach, it is assumed that the incident power is fed to the array at one of the two feeding ports while the other one is terminated with a matched load to suppress reflected signals. By means of this model, the amplitude and phase progressions of mm-wave signals which are distributed and coupled to each patch resonator are computed and used for calculating the overall radiation pattern. In order to compute these values as accurately as possible, electrical parameters listed in Fig. 3.1, should be correctly computed. Based on the electrical and physical parameters of the dielectric substrate and the transmission line, these parameters are computed in the next section using the closed-form expressions that have been derived and verified by other authors in the literature. Afterwards, these parameters are used to compute the propagation and distribution of the signals in a series fed array. After the verification of distribution via 
a full-wave simulator, the signals, whose amplitude and phase values can be controlled independently, are fed to the series feed array from opposite ports. Finally, the radiation patterns generated by these two independent signals are summed up for the overall desired radiation pattern.

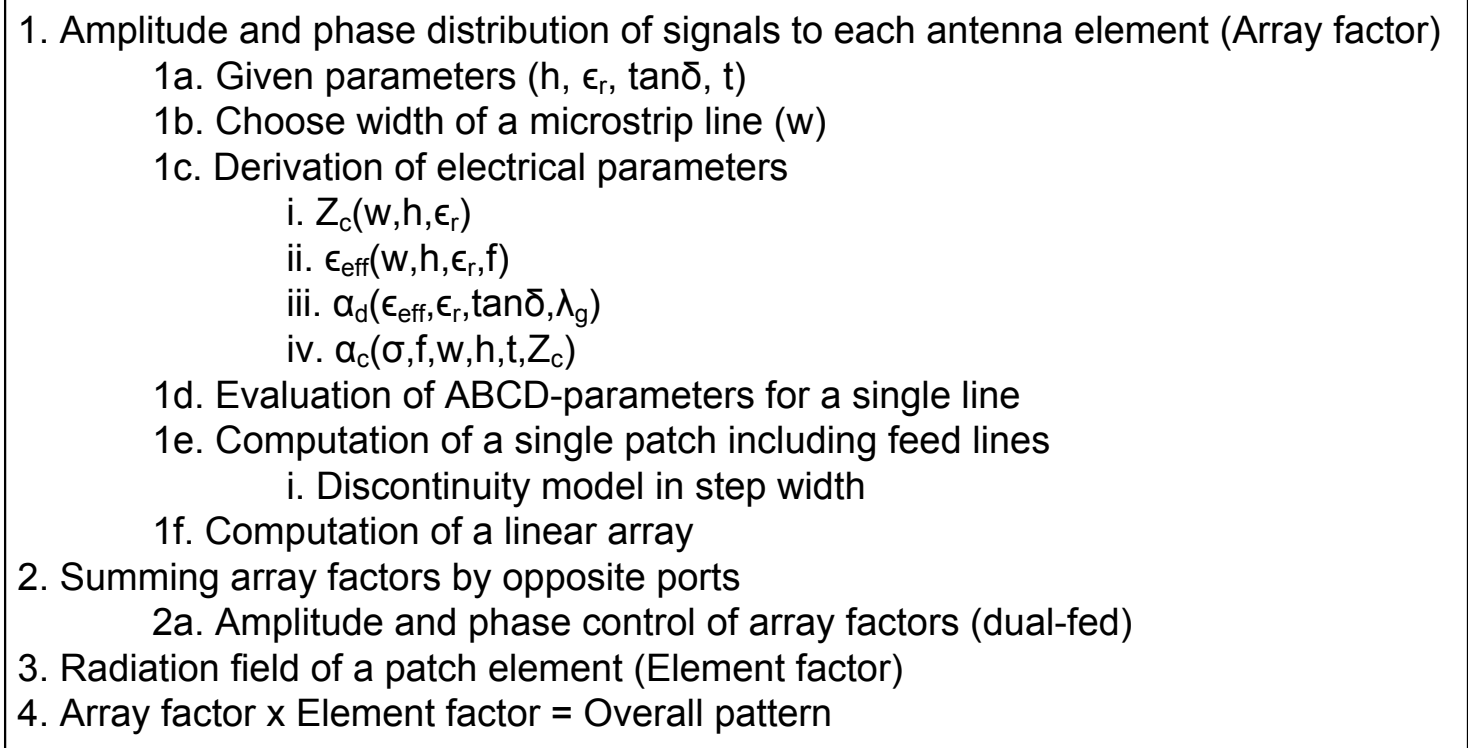

Figure 3.1.: The design flow.

The microstrip linear array consists of rectangular patch resonators. These resonators are connected to each other via high impedance lines to minimize the parasitic radiations (cross polarized patterns). These high impedance lines introduce a progressive phase shift between subsequent resonators that steers the main beam in different directions in the E-plane. The thickness of the dielectric substrate was chosen to be thin enough to avoid surface wave modes which are excited in thicker substrates. In order to improve the radiation efficiency, the relative dielectric constant of the substrate should be as low as possible. Therefore, the planar antenna is fabricated on a 127 um thick organic substrate with a dielectric permittivity $\epsilon_{r}=3[30]$.

In this work, a method-of-moments (MoM) based electromagnetic simulator is used to show validity of analytical model [35]. The method-of-moments technique only discretizes metal interconnects in the simulated model because the current distribution on the metal surfaces emerges as the core unknowns. Hence, MoM based simulators are best suited for the analysis of planar structures in terms of computational time compared to finite element method (FEM) or finite difference time domain (FDTD) based simulation tools.

In [36], an analytical model is presented for microstrip traveling wave array antennas. In that work, the microstrip patches are modeled as two radiating slots separated by a low impedance 
microstrip line. Microstrip step discontinuities which contribute an additional phase shift are not taken into account in this model. Also, the dispersion effect is neglected in this modeling. These omissions lead to deviations in the beam direction, beam shape and sidelobe levels of antenna characteristics. That being said, discontinuities at the junctions and dispersion effect must be taken into account in the analytical model for a more accurate simulation.

\subsection{Analytical model for a mm-wave microstrip linear array}

Fig. 3.1 shows a detailed design flow of the derived analytical model. Firstly, the electrical parameters of a microstrip line structure are expressed in terms of electrical and physical parameters specified by the chosen dielectric substrate and a conductor trace. The derived parameters are the characteristic impedance $\left(Z_{c}\right)$, the attenuation due to conductor $\left(\alpha_{c}\right)$ and dielectric losses $\left(\alpha_{d}\right)$, the guide wavelength $\left(\lambda_{g}\right)$ and the propagation constant $(\beta)$. After the calculation of these parameters, an analysis of an antenna array can be carried out using the circuit representation of the distributed elements. This can be done using an ABCD matrix, or transfer matrix representation of line sections, shunt conductances as shown in [37]. By using these values, the $\mathrm{S}$-parameters of a straight microstrip line are evaluated. In the analytical model, a patch resonator is represented by a length of a microstrip line whose width is equal to that of a patch. Therefore, a single patch connected with 2 feed lines is computed by cascading the microstrip lines with different widths. However, the discontinuity due to step in width (for example a discontinuity in width between a feed line and a patch) has to be taken into account since it leads to extra phase shift and insertion loss. Using this method, positions, excitation amplitudes and phases of all patch elements can be specified to compute the array factor in a series-fed linear array. Effects of a variable gain amplifier and a phase shifter are added to this array factor. Finally, the array factors, supplied by opposite ports, are then added to each other and multiplied with an element factor to obtain the overall radiation pattern.

An overall pattern is obtained via the multiplication of the array factor and the element factor, where the element factor is the radiation field of the microstrip patch antenna in this work. If an array is fed by both ports, the array factors due to both of the ports can be added. The phase difference and amplitude ratio of both signals can be adjustable in these array factors.

\subsubsection{Computation of electrical parameters}

Computation of effective dielectric permittivity $\left(\epsilon_{e f f}\right)$ : The effective dielectric constant of the microstrip line $\left(\epsilon_{e f f}\right)$ is calculated using different closed-form quasi-static and dispersion formulas in the literature. The quasi-static formulas, based on a TEM mode of propagation, can be used at lower frequencies. However, at higher frequencies, the effective dielectric constant and characteristic impedance of a microstrip line depends on frequency. If the operating 
frequency increases, the transmission line becomes more dispersive resulting in an increase in these values due to the propagation of hybrid modes (TE and TM modes) in addition to the fundamental quasi-TEM mode [38]. Therefore, frequency dispersion is taken into account in the designed model. 3 different well-known dispersion models are explained here in terms of their accuracies and applicable parameter ranges.

In the dispersion formulas, the effective dielectric constant is expressed in terms of frequency $(f)$, height of the substrate $(h)$, width of the microstrip line $(w)$, relative dielectric permittivity $\left(\epsilon_{r}\right)$ and effective dielectric permittivity $\left(\epsilon_{e f f}(f=0)\right)$. The quasi-static effective dielectric constant $\left(\epsilon_{e f f}(f=0)\right)$, given in [39], is used for computing $\epsilon_{e f f}$ in the next formulas. The quasi-static formula, derived by Hammerstad in [39], is a modified version of the equations, derived by Wheeler [40] and Schneider [41].

According to the dispersion model by E. Yamashita [42],[43], [44] the values deviate within $1 \%$ in a wide frequency range compared to the integral equation method used to derive the functional approximation. This approximate dispersion formula assumes the knowledge of the quasi-static effective dielectric constant $\left(\epsilon_{e f f}(f=0)\right)$. According to his paper, the applicable ranges of the formula are $2<\epsilon_{r}<16,0.06<w / h<16$ and $0.1 G H z<f<100$ $\mathrm{GHz}$, where $\epsilon_{r}$ is the relative dielectric permittivity of the substrate, $w$ the width of the strip, $h$ the height of the substrate and $f$ the operating frequency.

$$
\epsilon_{e f f}=\left(\frac{\sqrt{\epsilon_{r}}-\sqrt{\epsilon_{e f f}(f=0)}}{1+4 F^{-1.5}}+\sqrt{\epsilon_{e f f}(f=0)}\right)^{2},
$$

where

$$
F=\frac{4 h f \sqrt{\epsilon_{r}-1}}{\lambda_{0}}\left[0.5+\left\{1+2 \log \left(1+\frac{w}{h}\right)\right\}^{2}\right]
$$

The microstrip dispersion formula given by Kirschning and Jansen in [45] is based on Jansen's numerical hybrid mode technique. This formula provides accuracy better than $0.6 \%$ in the range of $0.1<w / h<100,1<\epsilon_{r}<20$ and $0<h / \lambda_{0}<0.13$, i.e. up to about $60 \mathrm{GHz}$ for $25 \mathrm{~mm}$ substrates.

$$
\begin{gathered}
\epsilon_{e f f}=\epsilon_{r}-\left(\frac{\epsilon_{r}-\epsilon_{e f f}(f=0)}{1+P(f)}\right) \\
P(f)=P_{1} P_{2}\left\{\left(0.1844+P_{3} P_{4}\right) 10 f h\right\}^{1.5763},
\end{gathered}
$$

where

$$
\begin{gathered}
P_{1}=0.27488+\frac{w}{h}\left[0.6315+\frac{0.525}{(1+0.157 f h)^{20}}\right]-0.065683 \exp \left(-8.7513 \frac{w}{h}\right) \\
P_{2}=0.33622\left[1-\exp \left(-0.03442 \epsilon_{r}\right)\right] \\
P_{3}=0.0363 \exp \left(-4.6 \frac{w}{h}\right)\left\{1-\exp \left(-(f h / 3.87)^{4.97}\right)\right\} \\
P_{4}=1+2.7511-\exp \left[-\left(\frac{\epsilon_{r}}{15.916}\right)^{8}\right]
\end{gathered}
$$


The dispersion model presented by Kobayashi [46], derived by comparison to a numerical model, has an accuracy better than $0.6 \%$ in the range $0.1 \leqslant w / h \leqslant 10,1<\epsilon_{r}<128$ for any $h / \lambda_{0}$ without frequency limit. $\epsilon_{e f f}(f=0)$ is the effective relative permittivity when the frequency is zero.

$$
\varepsilon_{r}(f)=\varepsilon_{r}-\frac{\varepsilon_{r}-\varepsilon_{e f f}(f=0)}{1+\left(\frac{f}{f_{50}}\right)^{m}}
$$

where

$$
\begin{gathered}
f_{50}=\frac{c_{0}}{2 \pi h\left(0.75+\left(0.75-\frac{0.332}{\epsilon_{r}^{1.73}}\right) \frac{w}{h}\right)} \cdot \frac{\arctan \left(\epsilon_{r} \sqrt{\frac{\epsilon_{e f f}(f=0)-1}{\epsilon_{r}-\epsilon_{e f f}(f=0)}}\right)}{\sqrt{\varepsilon_{r}-\varepsilon_{\text {eff }}(f=0)}} \\
m=m_{0} m_{c} \\
m_{0}=1+\frac{1}{1+\sqrt{\frac{w}{h}}}+0.32 \cdot\left(\begin{array}{l}
\left.\frac{1}{1+\sqrt{\frac{w}{h}}}\right)^{3} \\
m_{c}=\left\{\begin{array}{l}
1+\frac{1.4}{1+\frac{w}{h}}\left(0.15-0.235 \exp \left(\frac{-0.45 f}{f_{50}}\right)\right) \\
1
\end{array} \quad \text { for } w / h \leq 0.7\right. \\
1
\end{array}\right.
\end{gathered}
$$

The results of the analytical model employing these three dispersion models will be compared with the full-wave simulation results of a straight microstrip line in the next subsection. The optimum one will be selected for use in the analytical model.

Computation of characteristic impedance $\left(Z_{c}\right)$ : As stated in the previous section, dispersion also affects characteristic impedance. The characteristic impedance of a microstrip line is expressed in terms of width of line $(w)$, height of substrate $(h)$, frequency $(f)$ and effective dielectric constant of the substrate $\left(\epsilon_{e f f}\right)$. Since patches with different widths will be used for amplitude tapering, the characteristic impedances of these lines should be calculated correctly using the analytical model. Here, 3 different characteristic impedance models are provided for the comparison. These formulas are used for comparison purpose with the fullwave simulation results.

Wheeler in [40], formulated his synthesis and analysis equations for characteristic impedance based on a conformal mapping's approximation of the dielectric boundary with parallel conductor strips separated by a dielectric sheet. The formulas are applicable to alumina-type substrates $\left(8<\epsilon_{r}<12\right)$ and have an estimated relative error of less than $1 \%$.

The formulas by Schneider in [41], obtained by rational function approximation, give an accuracy of $\pm 0.25 \%$ for $0 \leq w / h \leq 10$, which is the range of importance. The accuracy 
obtained for strips with $w / h>10$ is $\pm 1 \%$.

Equations for the single microstrip line presented by Hammerstad in [47] are based upon an equation for the impedance of microstrip in an homogeneous medium and an equation for the microstrip effective dielectric constant. The obtained accuracy gives errors at least less than those caused by physical tolerances and is better than $0.01 \%$ for $w / h<1$ and $0.03 \%$ for $w / h \leq 1000$.

\subsubsection{Analysis of a straight microstrip line}

After evaluating the closed-form expressions for the characteristic impedance $Z_{c}$ and effective dielectric permittivity $\epsilon_{e f f}$, the transmission characteristics of microstrip lines with different widths and lengths are compared by employing different combinations of these formulas. In this section, these closed form expressions, which have been explained in the previous sections, are compared with full-wave simulation results in order to use the most optimum formulas for the next steps in the design process. The most important parameters to estimate the correct amplitude and phase distributions in a linear array antenna depends on the transmission characteristics of the structure $\left(S_{21}(d e g)\right.$ and $\left.S_{21}(d B)\right)$. The effect of return losses are neglected in this model since addition of each return loss at each discontinuity increases the complexity of the model.

Since each signal conductor, having a different width and length will be treated as a two-port network in the analytical model, a $2 \times 2$ transfer, or ABCD, matrix will be defined for each two-port network. Fig. 3.2 shows a two-port network of a microstrip line with a length of $l$ and a width of $w$. Using the ABCD matrix of each microstrip line, two or more two-port networks can be cascaded.

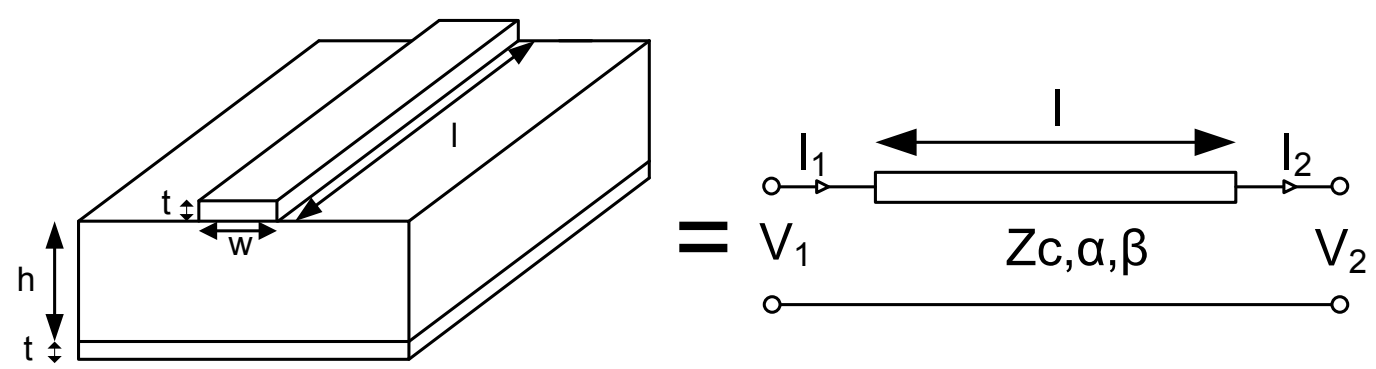

Figure 3.2.: A circuit model of a microstrip line.

$\mathrm{ABCD}$ parameters of this 2-port microstrip line can be found via the following formula;

$$
\left(\begin{array}{l}
V_{1} \\
I_{1}
\end{array}\right)=\left(\begin{array}{ll}
A & B \\
C & D
\end{array}\right)\left(\begin{array}{l}
V_{2} \\
I_{2}
\end{array}\right)
$$




$$
\left(\begin{array}{c}
V_{1} \\
I_{1}
\end{array}\right)=\left(\begin{array}{cc}
\cosh \gamma l & Z_{c} \sinh \gamma l \\
\frac{1}{Z_{c}} \sinh \gamma l & \cosh \gamma l
\end{array}\right)\left(\begin{array}{c}
V_{2} \\
I_{2}
\end{array}\right)
$$

The complex propagation constant $\gamma$ is given by

$$
\gamma=\alpha+j \beta
$$

where $\alpha$ is the attenuation factor and $\beta$ is the (real) propagation constant given by

$$
\beta=\frac{2 \pi}{\lambda_{g}}
$$

where $\lambda_{g}$ is the guide wavelength of a microstrip line, computed using the following equation:

$$
\lambda_{g}=\frac{\lambda_{0}}{\sqrt{\epsilon_{e f f}}}
$$

$\epsilon_{\text {eff }}$ is the effective dielectric constant, explained in Section 3.2.1. The attenuation factor, $\alpha$, is computed using formula:

$$
\alpha=\alpha_{c}+\alpha_{d}+\alpha_{r}+\alpha_{s}
$$

where $\alpha_{c}$ refers to conductor losses, $\alpha_{d}$ to dielectric losses, $\alpha_{r}$ to radiation losses and $\alpha_{s}$ to surface wave and higher order modes. In the designed model, since $\alpha_{r}$ and $\alpha_{s}$ are small, two other sources of losses are considered: conductor loss $\left(\alpha_{c}\right)$ and dielectric loss $\left(\alpha_{d}\right)$. The formulas, derived in [48], are used for the model.

After evaluating the ABCD parameters of microstrip lines, they can be easily converted to S-parameters. The model is used for computing the amplitude and phase values of the transmission coefficients in different microstrip lines by using the different formulas for the effective dielectric constants and characteristic impedances. According to the comparisons between the model and the full-wave simulations, the combination of the impedance equation by Wheeler and the effective dielectric constant by Yamashita provides the most accurate results. Fig. 3.3 shows amplitude and phase errors of the derived model at the different frequencies using these expressions. Within the desired frequency band, the maximum phase error is $2.7^{\circ}$ and the maximum amplitude error stays within $0.144 \mathrm{~dB} / \mathrm{cm}$.

These three formulas are compared with the full-wave simulation results in the next subsection and one of them is selected for use in the analytical model.

\subsubsection{Analysis of a single patch antenna including feed lines}

In this section, a patch element including feed lines on both sides, shown in Fig. 3.4, is expressed in terms of the transmission line model, which has been derived in the previous section. The ABCD matrices of each feed line and patch element are cascaded and multiplied with each other. 
(a) Amplitude errors

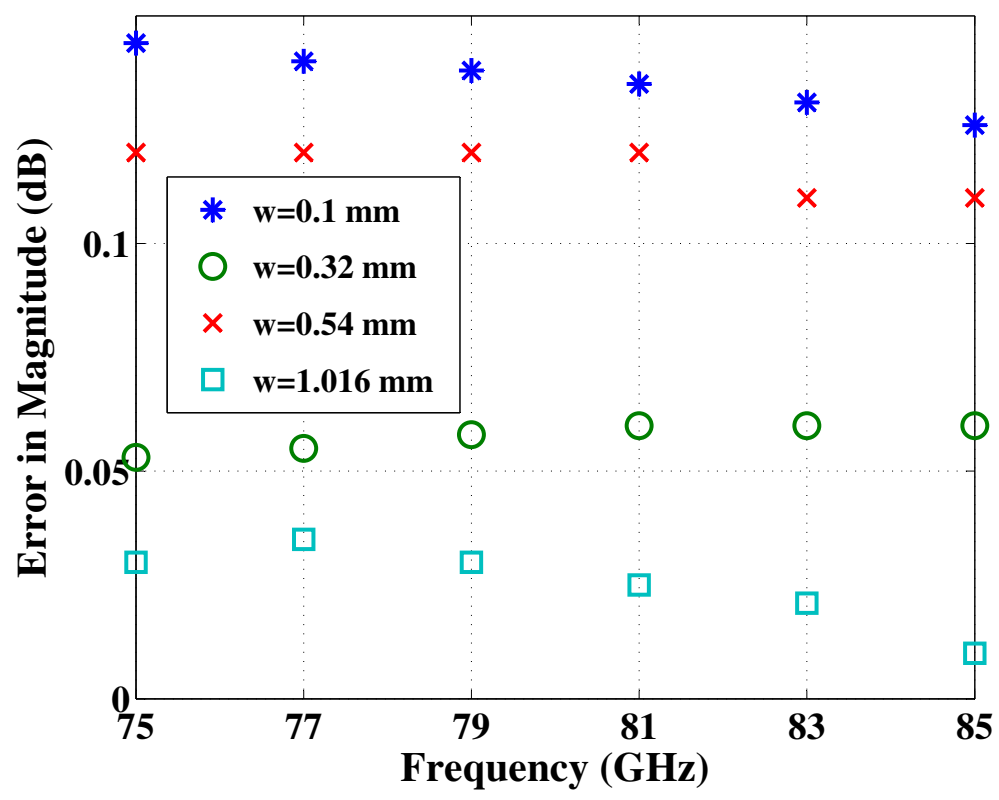

(b) Phase errors

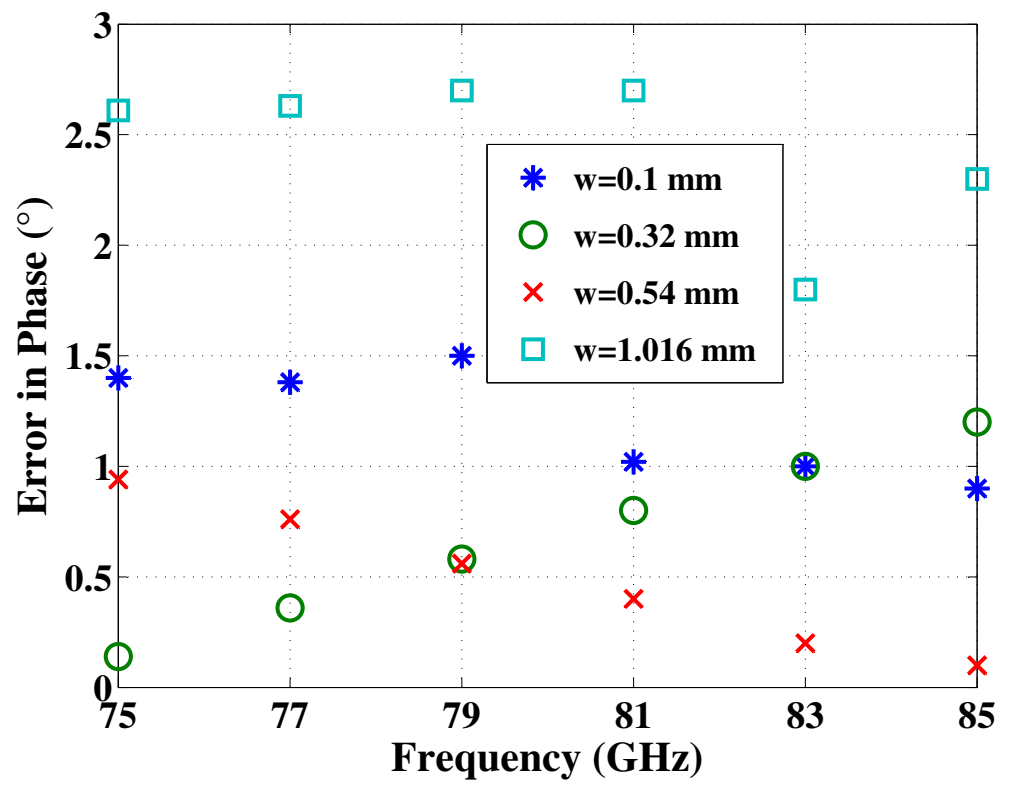

Figure 3.3.: Amplitude and phase errors of analytical model compared to full-wave simulation using straight microstrip lines with different widths $(w)$, as shown in Fig. 3.2.

The computation of the effective patch length is required. The resonant length of the patch is not exactly equal to the physical length due to fringing effects. The fringing effects cause the effective electrical length of the patch to be longer than its physical length. The two slots are separated by an electrical distance of $180^{\circ}$. However, because of the fringe effect the physical length $L$ becomes slightly less than $\lambda_{g} / 2$. Typically, it is $0.48 \lambda \leq L \leq 0.49 \lambda$ [27]. 


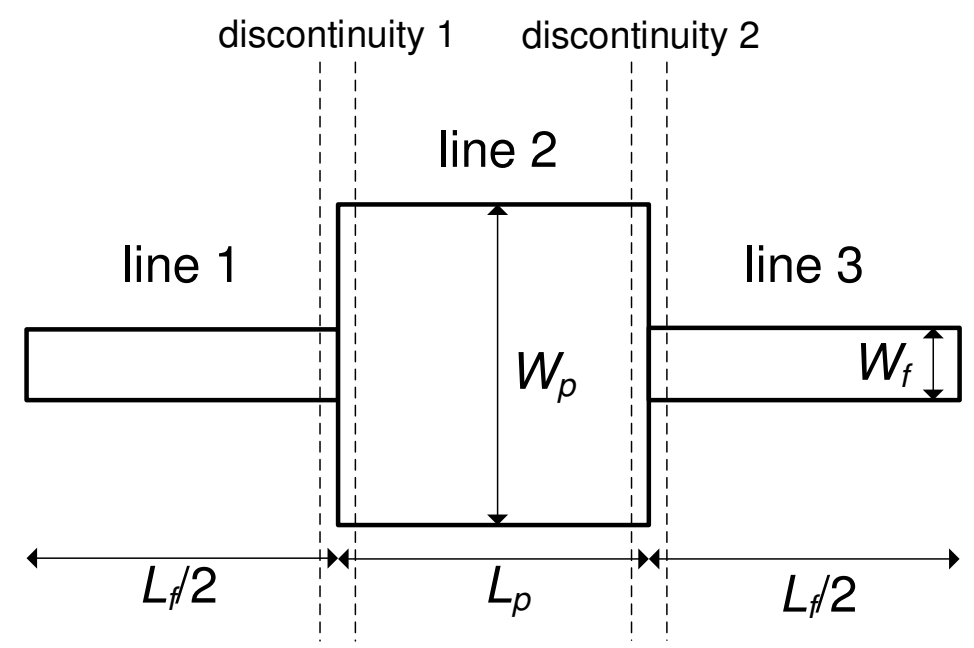

Figure 3.4.: A single patch antenna interconnected with feed lines.

Firstly, the model shown in Fig. 3.4 was computed without considering the discontinuity effects and compared with the full-wave simulations. The first model results were not accurate for different patch elements having different widths. An important fact was observed from these comparisons. The phase difference between the elements was much larger than could be accounted for by simple line length calculations. This phase difference increased linearly with the patch width, showing that junction discontinuities introduce an added phase shift which is proportional to the width of the element.

Therefore, the step in conductor width needs to be included to the analytical model. Each discontinuity contribute to an extra ABCD matrix. Then, the new equation is described as follows;

$$
\left(\begin{array}{ll}
A & B \\
C & D
\end{array}\right)=\left(\begin{array}{ll}
A & B \\
C & D
\end{array}\right)_{\text {line } 1}\left(\begin{array}{ll}
A & B \\
C & D
\end{array}\right)_{\text {dis.1 }}\left(\begin{array}{cc}
A & B \\
C & D
\end{array}\right)_{\text {line } 2}\left(\begin{array}{ll}
A & B \\
C & D
\end{array}\right)_{\text {dis. } 2}\left(\begin{array}{ll}
A & B \\
C & D
\end{array}\right)_{\text {line } 3}
$$

The field discontinuities in step width are due to the increase in current density from the wider to narrower conductor due to scattered electric fields at the front end of the wider conductor [49]. The current compression generates a series inductance $L_{s}$, and the scattered fields generate a parallel capacitance, $C_{p}$. $L_{s}$ can be computed using the formula given by [50]. The equivalent circuit of $C_{p}$ can be transformed into an equivalent length of transmission line $(\delta l)$. In terms of distributed elements, the discontinuity capacitance has the effect of an increase in the wide line's length and an equal decrease in the narrow line's length, as shown in Fig. 3.5.

The most accurate static equations are given using functional approximations of the low frequency calculations from a rigorous hybrid mode full-wave analysis [51]. For $t=0$ (thickness of metal trace), the equations are;

$$
\frac{\Delta l}{h}=\frac{A \cdot C \cdot E}{D}
$$




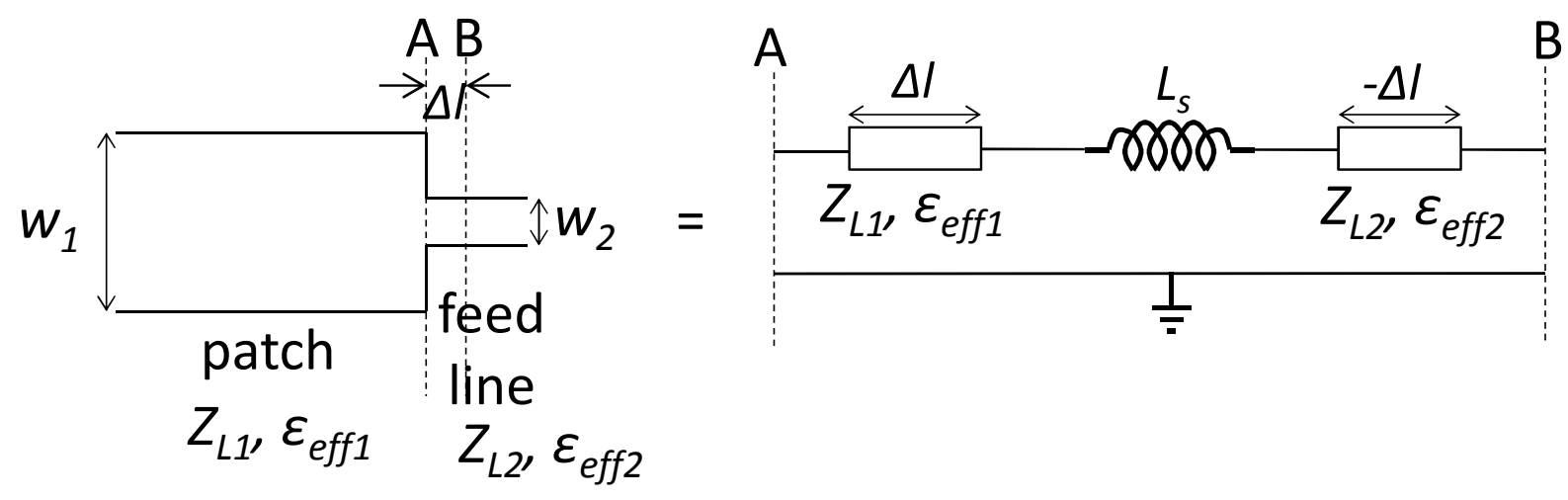

Figure 3.5.: A microstrip step discontinuity and its equivalent circuit.

$$
\begin{gathered}
A=0.434907 \frac{\epsilon_{e f f}^{0.81}+0.26 \cdot(w / h)^{0.8544}+0.236}{\epsilon_{\text {eff }}^{0.81}-0.189(w / h)^{0.8544}+0.87} \\
B=1+\frac{(w / h)^{0.371}}{2.358 \epsilon_{r}+1} \\
C=1+\left(0.5274 / \epsilon_{e f f}^{0.9236}\right) \cdot \arctan \left(0.084 \cdot(w / h)^{1.9413 / B}\right) \\
D=1+0.0377 \cdot\left[6-5 \exp \left(0.036\left(1-\epsilon_{r}\right)\right)\right]+\arctan \left(0.067 \cdot(w / h)^{1.456}\right) \\
E=1-0.218 \cdot \exp (-7.5 w / h),
\end{gathered}
$$

where the effective dielectric constant is given in [52] The error for $0.01 \leq w / h \leq 100$ and $1 \leq \epsilon_{r} \leq 50$ is less than $2.5 \%$.

After embedding the continuity model into the main analytical model, the model simulations are carried out and the results are compared with full-wave simulation results. By keeping the length of feed lines interconnected with the patch element constant, only the width of the patch element is changed to test the accuracy of the designed model. Fig. 3.6 shows the phase and amplitude errors of the model. The maximum phase error is within $3.88^{\circ}$ and the maximum amplitude error is within $1.22 \mathrm{~dB} / \mathrm{cm}$ range.

\subsubsection{Analysis of a microstrip linear array antenna}

After calculating the transfer matrix of each patch element connected with both feed lines, as discussed in the previous section, they are cascaded to each other to form multiple patch elements. By means of the cascaded models, the phase and amplitude distributions of a signal can be evaluated correctly. An aperture model, derived in [53], is used for calculating the radiation field of a rectangular microstrip resonator antenna (Fig. 3.7). The far field of a patch antenna is expressed with the following formula: 
(a) Amplitude errors

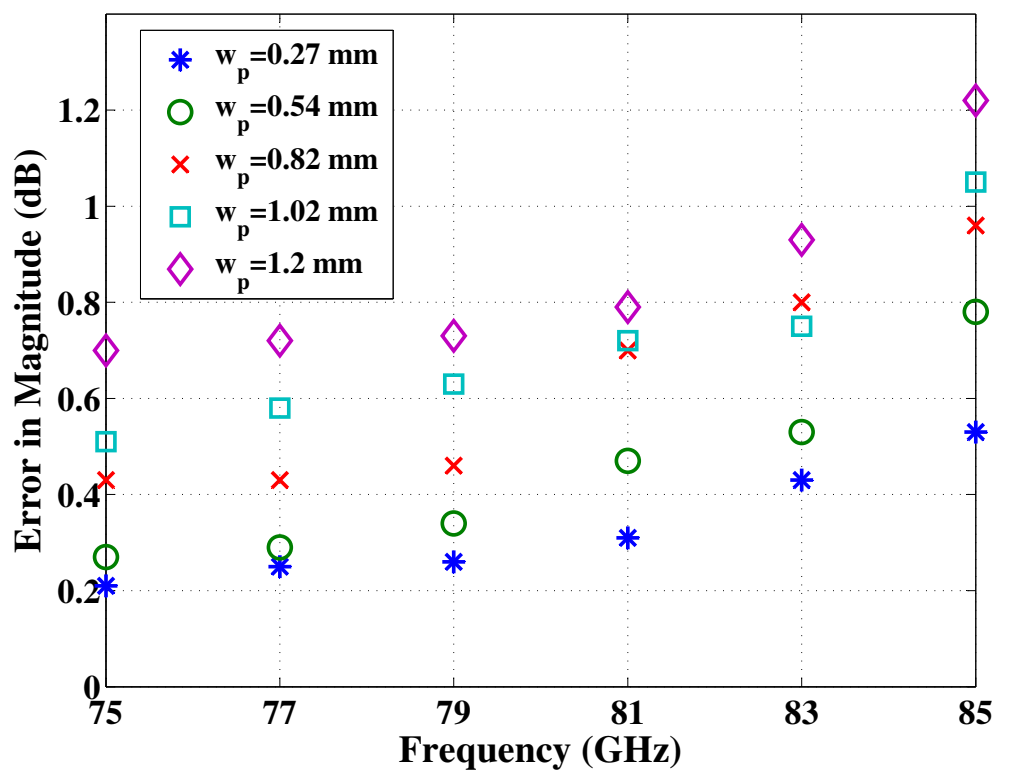

(b) Phase errors

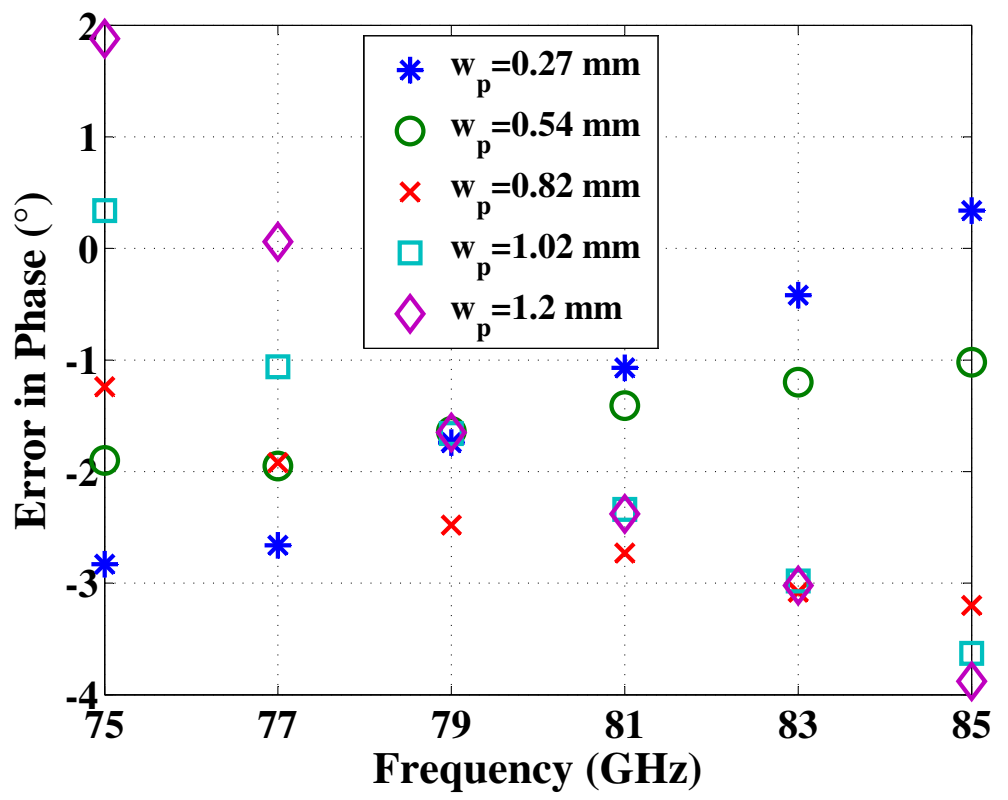

Figure 3.6.: Amplitude and phase errors of analytical model compared to the full-wave EM simulation using patch elements with different patch widths $\left(w_{p}\right)$ connected with feed lines, as shown in Fig. $3.4\left(L_{f}=5 \mathrm{~mm}\right)$.

$$
\begin{aligned}
E(r)= & E_{\theta} i_{\theta}+E_{\phi} i_{\phi} \\
= & \frac{j k \exp (-j k r)}{2 \pi r}\left[i_{\theta}\left(E_{x}(\xi, \eta) \cos \phi+E_{y}(\xi, \eta) \sin \phi\right)+\right. \\
& \left.i_{\phi}\left(-E_{x} \xi, \eta \sin \phi \cos \theta+E_{y}(\xi, \eta) \cos \phi \cos \theta\right)\right],
\end{aligned}
$$




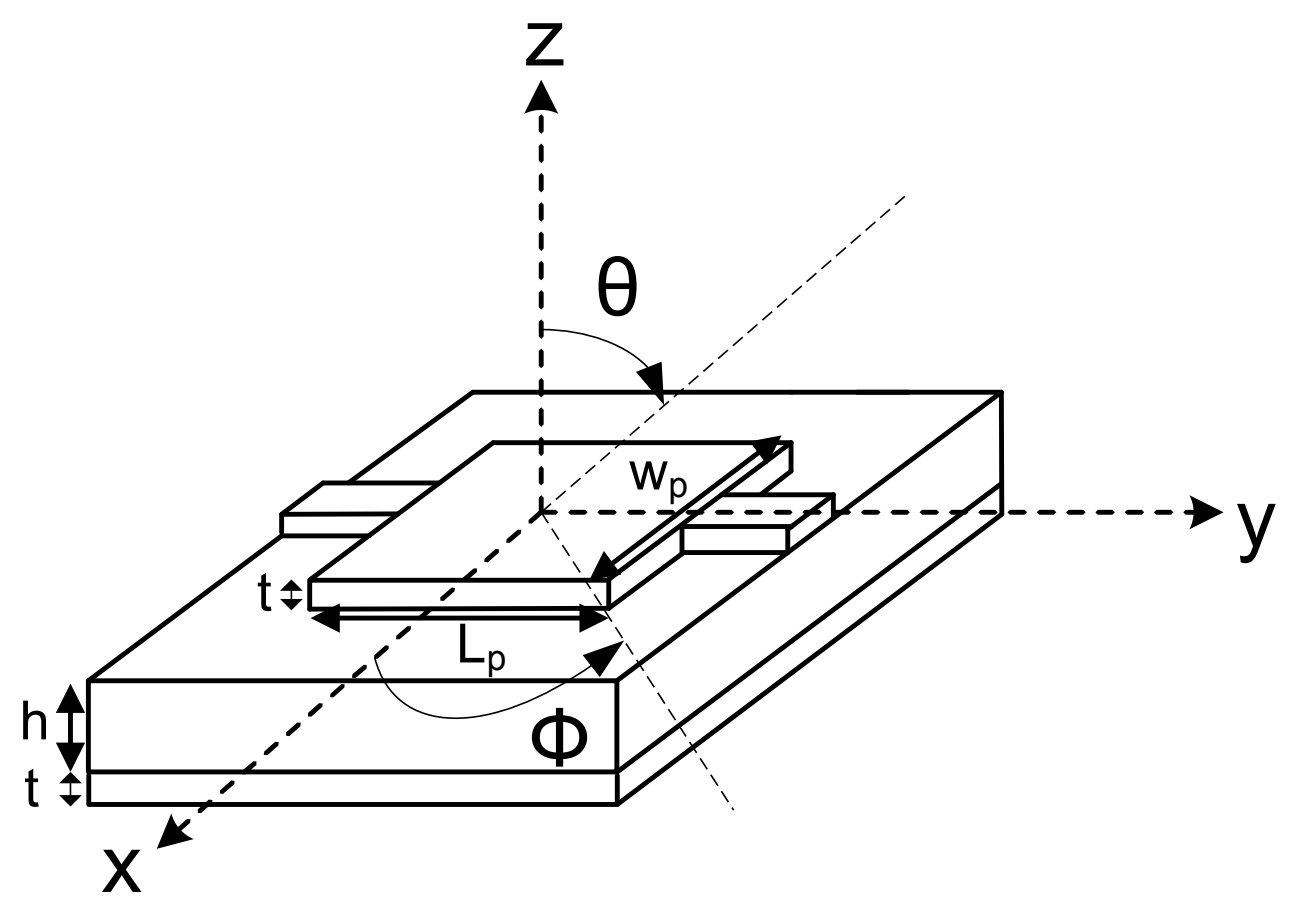

Figure 3.7.: Rectangular microstrip resonator antenna.

where

$$
\begin{aligned}
E_{x}(\xi, \eta)= & {\left[\left(-1-(-1)^{m}\right) j \sin (\xi b)+\left(1-(-1)^{m}\right) \cos (\xi b)\right] h E_{0} c \operatorname{sinc}(a \xi) j^{n} } \\
& {\left[\operatorname{sinc}\left(\eta c+\frac{n \pi}{2}\right)+(-1)^{n} \operatorname{sinc}\left(\eta c-\frac{n \pi}{2}\right)\right] } \\
E_{y}(\xi, \eta)= & {\left[\left(-1-(-1)^{n}\right) j \sin \eta c+\left(1-(-1)^{n}\right) \cos \eta c\right] h E_{0} b \sin c(a \eta) j^{m} } \\
& {\left[\operatorname{sinc}\left(\xi b+\frac{m \pi}{2}\right)+(-1)^{m} \operatorname{sinc}\left(\eta b-\frac{m \pi}{2}\right)\right] }
\end{aligned}
$$$$
\xi=k \sin \theta \cos \phi
$$

$$
\eta=k \sin \theta \sin \phi
$$

$$
k=\frac{2 \pi}{\lambda_{0}}
$$

$$
a=0.5 h
$$




$$
\begin{aligned}
& b=0.5 L_{p} \\
& c=0.5 w_{p}
\end{aligned}
$$

In these equations, $E_{0}$ is the maximum amplitude of the field, $h$ is the height of the substrate and $k$ is the wave number in free space. By means of Eq. 3.27, radiation patterns can be computed for every mode of the rectangular resonator, shown in Fig. 3.7. In this thesis, only fundamental mode $T M_{m n}(\mathrm{~m}=0, \mathrm{n}=1)$ is taken into account for the analytical patterns. The parameters, listed in Table A.1, are used for computation of radiation patterns using the analytical model.

The validity of analytical model is confirmed with an aid of a full-wave simulator, Agilent ADS, by computing different radiation patterns. Fig. 3.8 shows analytical and full-wave simulation results of a linear array antenna made up of 10 patch elements. This antenna is computed at $77 \mathrm{GHz}$. The feed line length between subsequent patch elements is varied in Fig. 3.8(a), Fig. 3.8(b) and Fig. 3.8(c).

Another linear array antenna, which is made up of 5 elements, is simulated at $77 \mathrm{GHz}$ for the verification of the analytical model in Fig. 3.9. It can be seen from this figure that the direction of the main beam and sidelobes can be computed almost correctly. The main beamwidth is also calculated correctly. However, the sidelobe levels are lower than simulated results. The increase in the feed line lengths tilts the main beam to broadside.

\subsubsection{Dual-fed linear array antenna}

Fig. 3.10 shows an analytical model for a linear array antenna fed by both ports. A variable gain amplifier and a phase shifter are connected to Port 1 to change the amplitude ratio and phase difference. The overall radiation pattern is obtained by using the following equation:

$$
R P(\theta)=\left(A * A F 1 * \exp \left(-j \phi_{p s}\right)+A F 2\right) * E F,
$$

where $R P$ refers to radiation pattern, $A$ to amplitude ratio between two ports, $\phi_{p s}$ to phase value of a phase shifter, $A F 1$ to array factor due to signal fed by port $1, A F 2$ to array factor due to signal fed by port 2, $E F$ to element factor, rectangular patch antenna in this case. Array factor can be found using:

$$
A F(\theta)=\sum_{n=1}^{m} a_{n} \exp \left(\frac{-j 2 \pi x_{n} \sin \theta}{\lambda_{0}}\right),
$$

where

$$
a_{n}=u_{n} S 21_{p s} \prod_{i=1}^{n-1} S 21_{i} k_{f, i}
$$




$$
\begin{gathered}
u_{n}=\sqrt{1-\left|S 21_{n}\right|^{2}} \\
k_{f, n}=\exp \left(-j 2 \pi L_{f, n} / \lambda_{g}\right) \\
x_{n}=(n-1)\left(L_{p}+L_{f}\right)
\end{gathered}
$$

After replacing Eq. 3.38, Eq. 3.39 and Eq. 3.41 into Eq. 3.37,

$$
A F(\theta)=\sum_{n=1}^{m}\left[\sqrt{1-\left|S 21_{n}\right|^{2}} \prod_{i=1}^{n-1} S 21_{i} k_{f, i}\right] \exp \left(\frac{-j 2 \pi\left((n-1)\left(L_{p}+L_{f}\right)\right) \sin \theta}{\lambda_{0}}\right)
$$

Eq. 3.42 shows the analytical approach of phase and amplitude distributions to each patch element in a linear array antenna, as shown in Fig. 3.10. This equation is used for calculating the radiation pattern of a signal fed by one of two ports. $\theta$ refers to the direction of the beam, $A F(\theta)$ to the array factor, $u_{n}$ to the radiated signal ignoring reflections at the discontinuities of the patches, $m$ to the number of patch elements, $L_{p}$ to the length of patches, $L_{f}$ to the length of feed lines between subsequent patch elements. If only one port is used for supplying the signal to the antenna, the amplifier and phase shifter will have no influence on beam steering. However, if the signals are supplied by both ports, a beam steering can be achieved by controlling values of $U$ and $\phi_{p s}$.

The linear array antenna is fed by opposite ports with $0^{\circ}$ and $180^{\circ}$ phase differences and same amplitude values. The analytical model is used for a linear array antenna made up of 10 patch elements. The length of feed lines $\left(L_{f}\right)$ is $1.25 \mathrm{~mm}$, which is slightly larger than $\lambda_{g} / 2$ at $77 \mathrm{GHz}$. If phase difference is $0^{\circ}$, a difference antenna pattern is generated, as shown in Fig. 3.11(a). If the difference is $180^{\circ}$, a sum antenna pattern is generated, as depicted in Fig. 3.11(b). These two figures, which compare analytical model and full-wave simulation results, validate the accuracy of the derived analytical model.

Although the directions of the minimum peaks in the patterns, shown in Fig. 3.11(b) and Fig. 3.11(a), can be computed correctly, the levels of maximum peaks are lower than the maximum peaks computed via a full-wave simulator. This example shows the computational accuracy of phase distribution throughout the linear array. However, the accuracy of amplitude distribution throughout the linear array must be improved further to compute the magnitude level of the peaks correctly. 
(a) $L_{f}=0.8 \mathrm{~mm}$ (feed line length between subsequent patches).

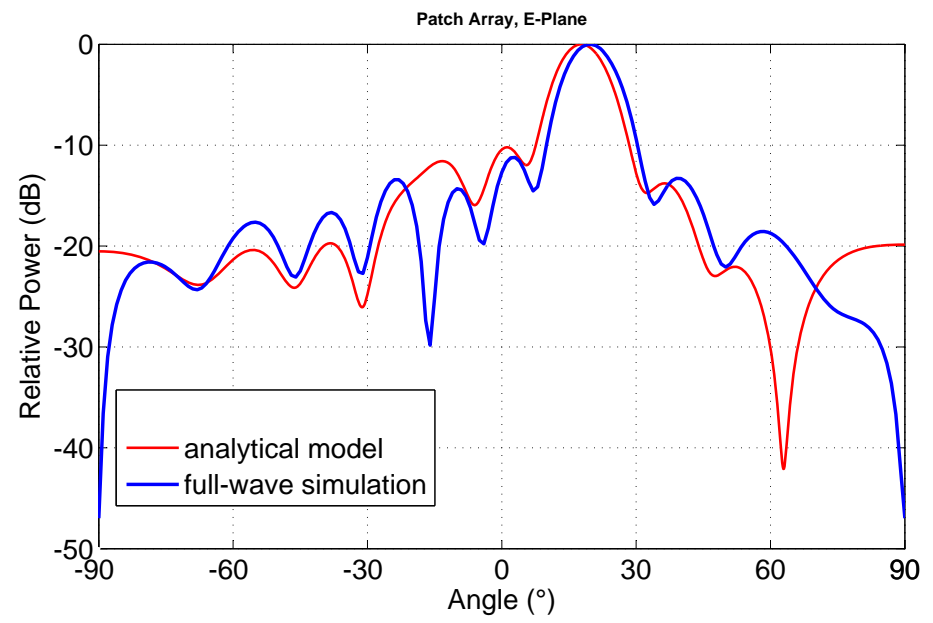

(b) $L_{f}=1.25 \mathrm{~mm}$ (feed line length between subsequent patches).

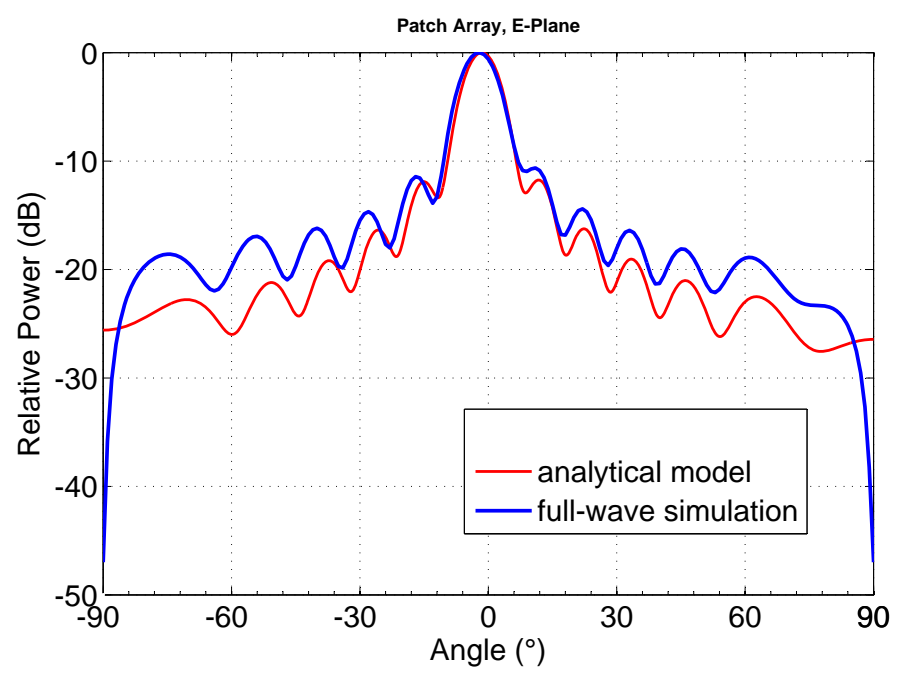

(c) $L_{f}=2 \mathrm{~mm}$ (feed line length between subsequent patches).

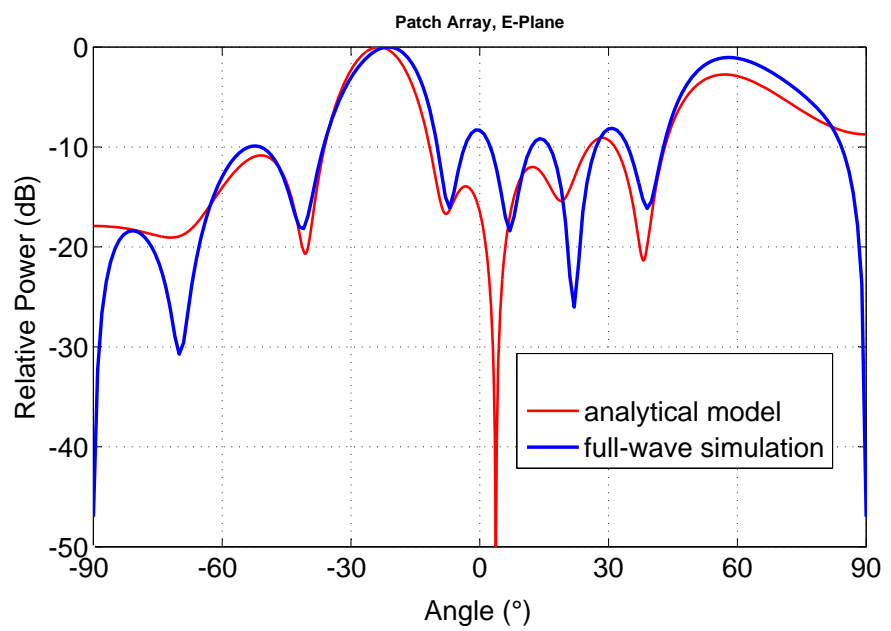

Figure 3.8.: Effect of feed line length on the radiation pattern of a linear array antenna (with $N=10)$. The antenna is fed by one port only. 
(a) $L_{f}=0.8 \mathrm{~mm}$ (feed line length between subsequent patches).

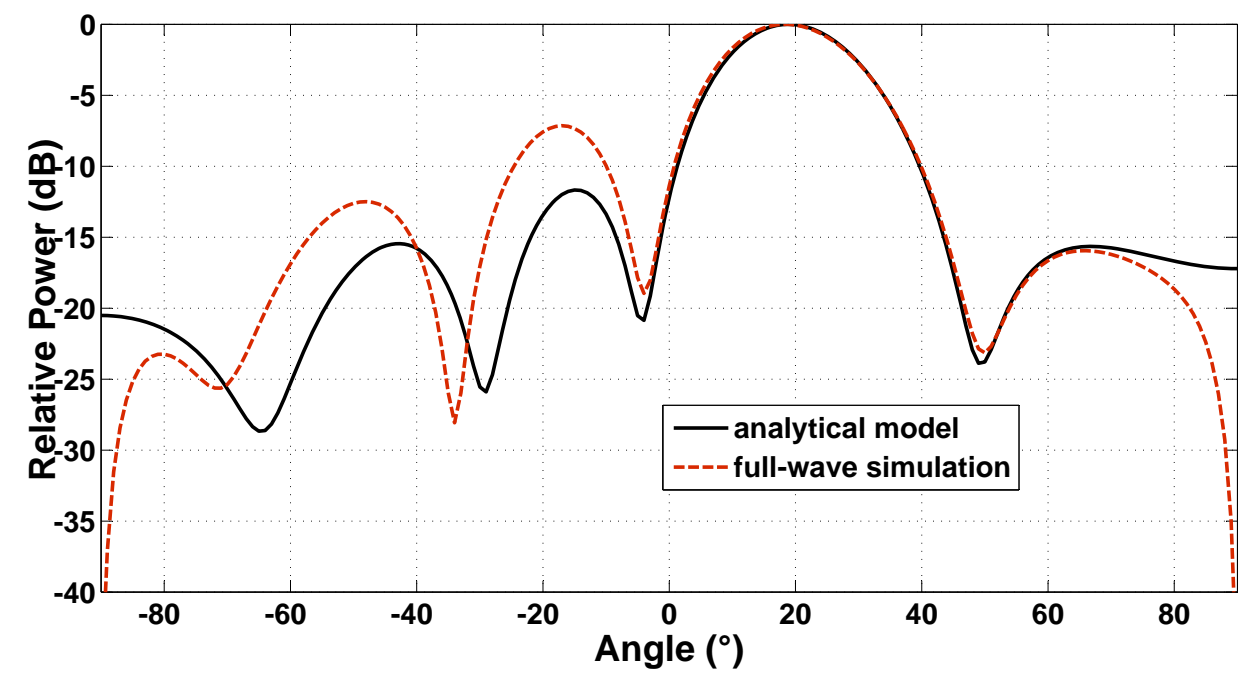

(b) $L_{f}=0.9 \mathrm{~mm}$ (feed line length between subsequent patches).

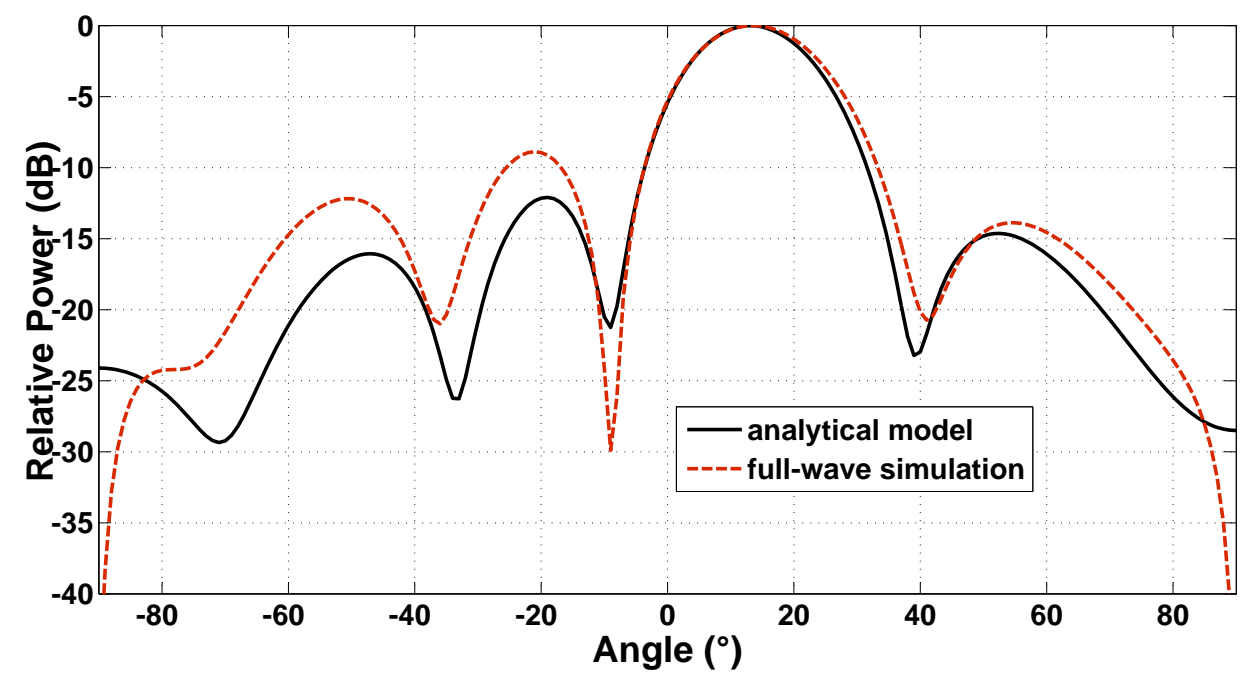

(c) $L_{f}=1.1 \mathrm{~mm}$ (feed line length between subsequent patches).

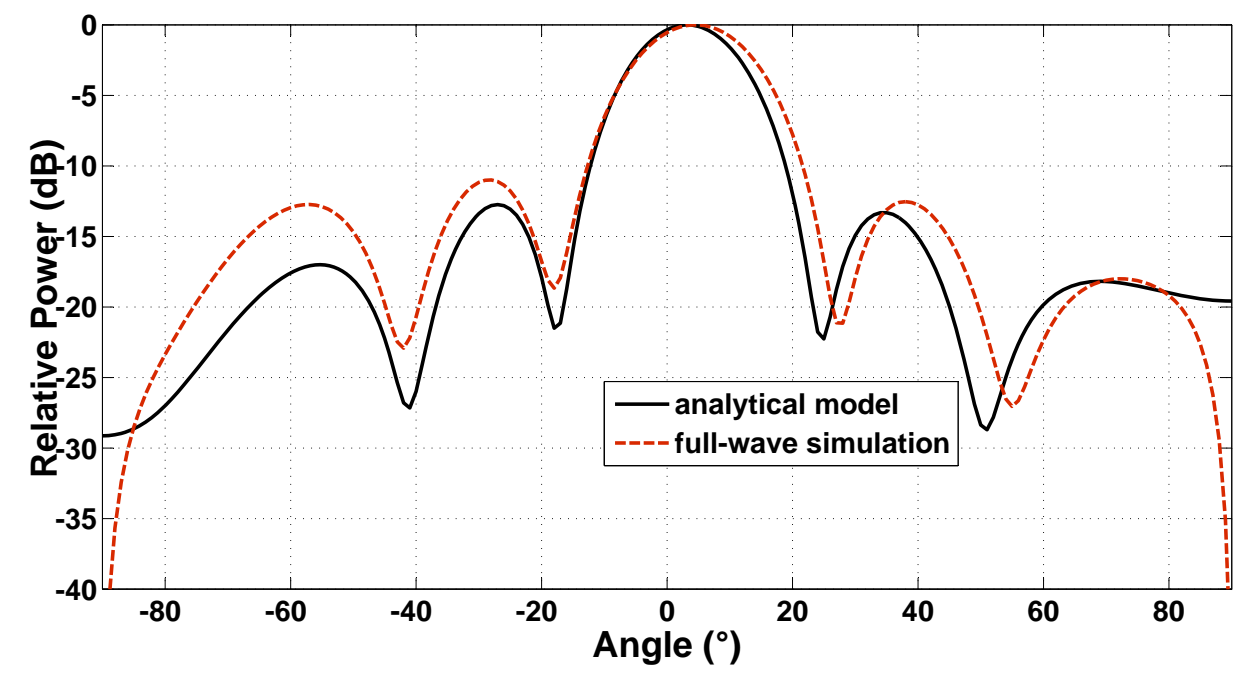

Figure 3.9.: Effect of feed line length on the radiation pattern of a linear array antenna (with $38 \quad N=5$ ). The antenna is fed by one port only. 


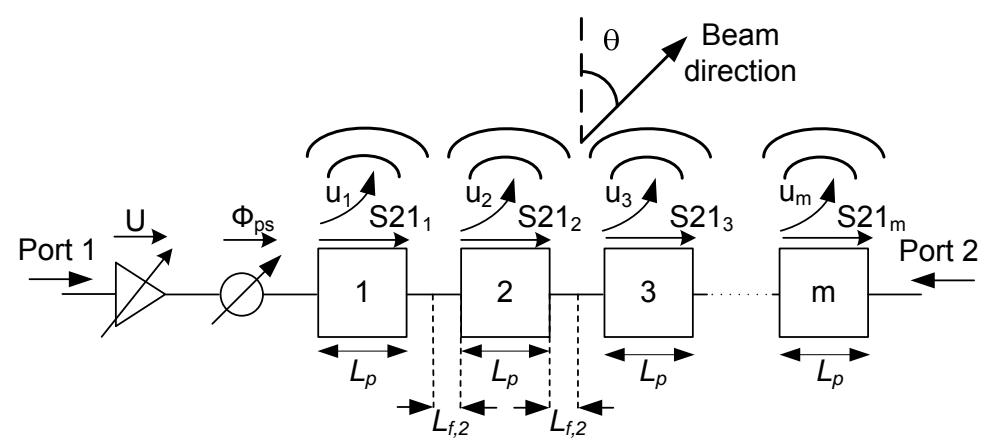

Figure 3.10.: Analytical model of a linear array antenna fed by both ports.

(a) The difference antenna pattern $\left(A 1: 0.707 \phi 1: 0^{\circ} A 2: 0.707 \phi 2\right.$ :

$\left.0^{\circ}\right)$.

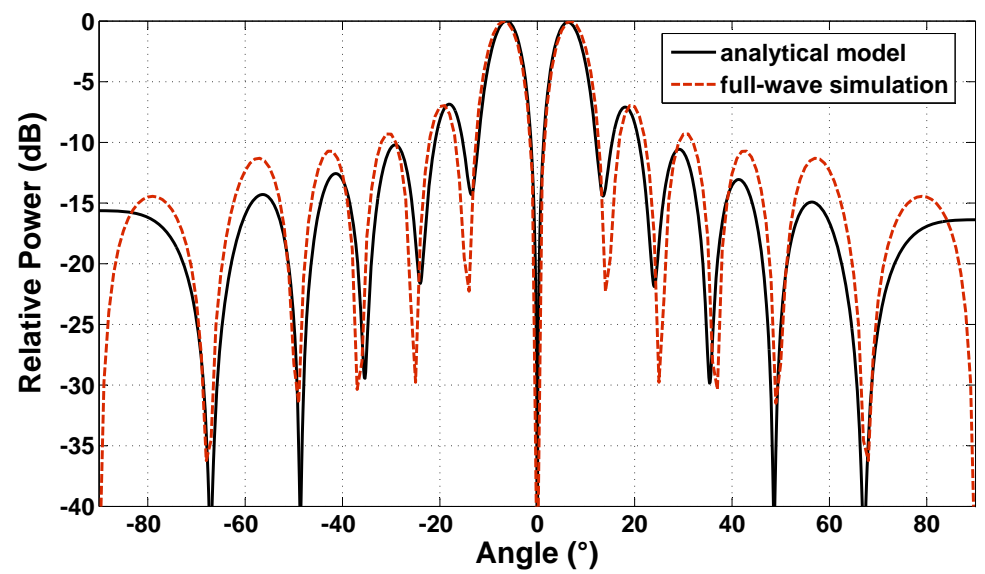

(b) The sum antenna pattern $\left(A 1: 0.707 \phi 1: 180^{\circ} A 2: 0.707 \phi 2: 0^{\circ}\right)$.

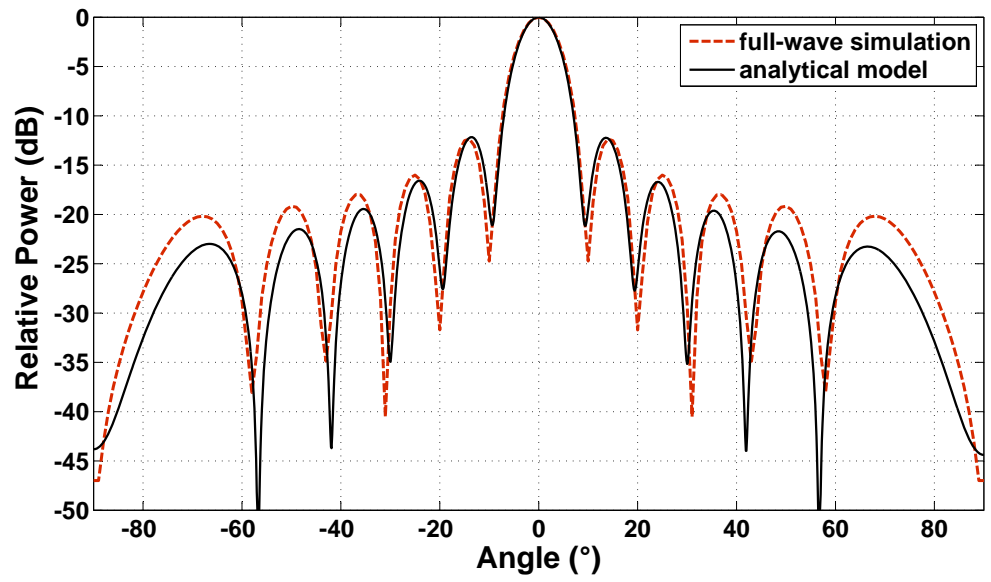

Figure 3.11.: Dual-fed linear array antenna consisting up of 10 patch elements $\left(L_{f}=1.25\right.$ $\mathrm{mm})$. 


\subsection{Investigation of design parameters}

The main goal of this subsection is to investigate effects of design parameters on the overall antenna performance. The investigated parameters are the number of patch elements and the length of feed lines between patch elements. Even though change of the operating frequency is not a design parameter and it is directly related with the length of feed lines, it is also analyzed in one of the subsections.

\subsubsection{Number of patch elements}

In this subsection, effect of number of patch elements on the radiation pattern is analyzed by using the derived analytical model. Linear arrays are computed using the analytical model at $77 \mathrm{GHz}$ employing $1.5 \mathrm{~mm}$ long feed lines $\left(L_{f}\right)$, which is larger than $\lambda_{g} / 2$ (Fig. 3.12). Like in the principle of a traveling wave antenna, a linear array antenna in Fig. 3.12(a) is fed only by one port whereas other port is terminated with a matched load to avoid the reflection of signals. The increase in number of patch elements causes an increase in the antenna aperture and consequently, the angle of the main beam to tilt towards the broadside of the antenna gradually, as shown in Table 3.1(a). The beamwidth of the main beam becomes narrower and the first sidelobe levels stay almost same.

Fig. 3.12(b) shows the radiation patterns of different arrays when the antenna is fed by both ports with same amplitude and phase values. It is seen from this figure that directions of dual beams are shifted towards the broadside of the antenna with an increase in number of patch elements. The beamwidths are narrower, whereas the sidelobe levels becomes higher, as shown in Table 3.1(b).

A main beam can also look into broadside direction if signals are fed through two ports with same amplitudes and a $180^{\circ}$ phase difference. In Fig. 3.12(c), a dual antenna beam appears again if 10 or 15 patches are used in array. An increase in the number of patches results in an increase in sidelobe levels (Table 3.1(c)).

In Fig. 3.13, linear arrays are computed using the analytical model and the radiation patterns are compared with each other in terms of the number of antenna elements at $77 \mathrm{GHz}$ employing a $1.25 \mathrm{~mm}$ long feed line length $\left(L_{f}\right)$, which is slightly larger than $\lambda_{g} / 2$. Table 3.2 shows the values of antenna parameters, computed in Fig. 3.13. It can be deduced from Table 3.2(a) that the reduction in length of the feed line $\left(L_{f}\right)$ results in a limited beam steering. The values for Table. 3.2(b) are almost same like in Table. 3.1(b). Only the sidelobe levels are just a few $\mathrm{dB}$ higher. A distinct change is observed in Table 3.2(c). The main beam becomes narrower and always looks at the broadside direction with an increase in the number of patches, as depicted in Fig. 3.13(c). 
(a) Fed only by one port ( $\left.A 1: 1 \phi 1: 0^{\circ} \& A 2: 0 \phi 2: 0^{\circ}\right)$.

\begin{tabular}{|c|c|c|c|}
\hline Number of patch elements & Beam direction $\left(^{\circ}\right)$ & Sidelobe levels $(d B)$ & HPBW $\left(^{\circ}\right)$ \\
\hline \hline 5 & -9 & 10.2 & 16 \\
\hline 10 & -8 & 10.4 & 8 \\
\hline 15 & -7 & 10.8 & 6 \\
\hline
\end{tabular}

(b) Difference pattern $\left(A 1: 0.707 \phi 1: 0^{\circ} \& A 2: 0.707 \phi 2: 0^{\circ}\right)$.

\begin{tabular}{|c|c|c|c|}
\hline Number of patch elements & Beam direction $\left(^{\circ}\right)$ & Sidelobe levels $(d B)$ & HPBW $\left(^{\circ}\right)$ \\
\hline \hline 5 & \pm 12 & 10.6 & 13 \\
\hline 10 & \pm 6 & 10.6 & 7 \\
\hline 15 & \pm 9 & 8.5 & 6 \\
\hline
\end{tabular}

(c) Sum pattern $\left(A 1: 0.707 \phi 1: 0^{\circ} \& A 2: 0.707 \phi 2: 180^{\circ}\right)$.

\begin{tabular}{|c|c|c|c|}
\hline Number of patch elements & Beam direction $\left(^{\circ}\right)$ & Sidelobe levels $(d B)$ & HPBW $\left(^{\circ}\right)$ \\
\hline \hline 5 & 0 & 21.8 & 19 \\
\hline 10 & \pm 9 & 8.56 & 6 \\
\hline 15 & \pm 7 & 8.2 & 4 \\
\hline
\end{tabular}

Table 3.1.: Effect of number of patch elements on an overall pattern $\left(L_{f}=1.5 \mathrm{~mm}\right)$.

(a) Fed only by one port ( $\left.A 1: 1 \phi 1: 0^{\circ} \& A 2: 0 \phi 2: 0^{\circ}\right)$.

\begin{tabular}{|c|c|c|c|}
\hline Number of patch elements & Beam direction $\left(^{\circ}\right)$ & Sidelobe levels $(d B)$ & HPBW $\left(^{\circ}\right)$ \\
\hline \hline 5 & 2 & 12.7 & 17 \\
\hline 10 & 1 & 11.3 & 9 \\
\hline 15 & 0.5 & 10.2 & 6 \\
\hline
\end{tabular}

(b) Difference pattern $\left(A 1: 0.707 \phi 1: 0^{\circ} \& A 2: 0.707 \phi 2: 0^{\circ}\right)$.

\begin{tabular}{|c|c|c|c|}
\hline Number of patch elements & Beam direction $\left(^{\circ}\right)$ & Sidelobe levels $(d B)$ & HPBW $\left(^{\circ}\right)$ \\
\hline \hline 5 & \pm 13 & 7.6 & 14 \\
\hline 10 & \pm 6 & 7.2 & 6 \\
\hline 15 & \pm 4 & 7.4 & 4 \\
\hline
\end{tabular}

(c) Sum pattern $\left(A 1: 0.707 \phi 1: 0^{\circ} \& A 2: 0.707 \phi 2: 180^{\circ}\right)$.

\begin{tabular}{|c|c|c|c|}
\hline Number of patch elements & Beam direction $\left(^{\circ}\right)$ & Sidelobe levels $(d B)$ & HPBW $\left(^{\circ}\right)$ \\
\hline \hline 5 & 0 & 13 & 17 \\
\hline 10 & 0 & 12.6 & 9 \\
\hline 15 & 0 & 11.8 & 5 \\
\hline
\end{tabular}

Table 3.2.: Effect of number of patch elements on an overall pattern $\left(L_{f}=1.25 \mathrm{~mm}\right)$. 


\subsubsection{Length of feed lines between patches}

In this subsection, the length of feed lines are varied. Fig. 3.14 shows beam patterns of a linear array with 10 patch elements employing different lengths of feed lines. Fig. 3.14(a) shows the change of the beam direction if the length is varied. In this case, the signal is fed only by one of the ports. If the feed line is shorter than $\lambda_{g} / 2$, the main beam is broader and looks to a direction in the same half of the field of view. The main beam looks almost at the broadside when the feed line length $\left(L_{f}\right)$ is almost equal to $\lambda_{g} / 2$ at $77 \mathrm{GHz}$ (Table 3.3(a)). If $L_{f}$ is larger than $\lambda_{g} / 2$, the main beam looks at the opposite half of the field of view with a narrower beamwidth because increase in an element spacing makes the antenna aperture of the whole array larger.

Fig. 3.14(b) shows effect of element spacings on difference beam patterns. If the element spacing is longer or shorter than $\lambda_{g} / 2$, directions of main beams looks away from the broadside. This effect makes dual beams narrower, as depicted in Table 3.3(b). The same is also valid for the sum patterns, as shown in Fig. 3.14(c). However, only line length of $1.2 \mathrm{~mm}$ can create a sum pattern, whereas other lengths still generate dual-beam patterns (Table 3.3(c)).

(a) Fed only by one port ( $\left.A 1: 1 \phi 1: 0^{\circ} \& A 2: 0 \phi 2: 0^{\circ}\right)$.

\begin{tabular}{|c|c|c|c|}
\hline Length of feed lines $(\mathrm{mm})$ & Beam direction $\left(^{\circ}\right)$ & Sidelobe levels $(\mathrm{dB})$ & HPBW $\left(^{\circ}\right)$ \\
\hline \hline 0.8 & 19 & 11.1 & 11 \\
\hline 1.2 & 1 & 10.9 & 9 \\
\hline 1.6 & -11 & 12.1 & 8 \\
\hline
\end{tabular}

(b) Difference pattern $\left(A 1: 0.707 \phi 1: 0^{\circ} \& A 2: 0.707 \phi 2: 0^{\circ}\right)$.

\begin{tabular}{|c|c|c|c|}
\hline Length of feed lines $(\mathrm{mm})$ & Beam direction $\left(^{\circ}\right)$ & Sidelobe levels $(\mathrm{dB})$ & HPBW $\left(\left(^{\circ}\right)\right)$ \\
\hline \hline 0.8 & \pm 20 & 10 & 10 \\
\hline 1.2 & \pm 6 & 6.7 & 9 \\
\hline 1.6 & \pm 12 & 9.6 & 6 \\
\hline
\end{tabular}

(c) Sum pattern $\left(A 1: 0.707 \phi 1: 0^{\circ} \& A 2: 0.707 \phi 2: 180^{\circ}\right)$.

\begin{tabular}{|c|c|c|c|}
\hline Length of feed lines $(\mathrm{mm})$ & Beam direction $\left(^{\circ}\right)$ & Sidelobe levels $(\mathrm{dB})$ & HPBW $\left(^{\circ}\right)$ \\
\hline \hline 0.8 & \pm 17 & 6.3 & 12 \\
\hline 1.2 & 0 & 12.5 & 9 \\
\hline 1.6 & \pm 10.5 & 8.2 & 7 \\
\hline
\end{tabular}

Table 3.3.: Effect of feed line length on an overall pattern $(N=10)$.

\subsubsection{Operating frequency}

The change of the operating frequency affects the relative phase shift between antenna elements, leading to change of beam direction. If the operating frequency increases, the guide 
wavelength will be shorter. This will result in an increase in electrical length of element spacings. In this subsection, a linear array antenna with 5 patch elements is simulated which has $0.8 \mathrm{~mm}$ long element spacings between its patches $\left(L_{f}\right)$. Fig. 3.15(a) and Table 3.4(a) show how the direction and beamwidth of the main beam vary if the frequency is increased from 75 to $81 \mathrm{GHz}$. The beam direction shifts from $22^{\circ}$ to $3^{\circ}$ with the increase in the frequency. Sidelobe levels are slightly lower and the beamwidth becomes narrower. At difference beam patterns, beamwidths and sidelobes of dual beams are affected by the change of frequency (Fig. 3.15(b)). The directions of the main beams shift to the broadside direction gradually and the beamwidth becomes narrower (Table 3.4(b)). In Fig. 3.15(c), sum patterns are shown for different frequencies. Only at $75 \mathrm{GHz}$, the dual beam pattern exists. For the other frequencies, the sum pattern can be obtained with different beamwidths, looking at the broadside direction.

(a) Fed only by one port $\left(A 1: 1 \phi 1: 0^{\circ} \& A 2: 0 \phi 2: 0^{\circ}\right)$.

\begin{tabular}{|c|c|c|c|}
\hline Operating frequency $(\mathrm{GHz})$ & Beam direction $\left(^{\circ}\right)$ & Sidelobe levels $(d B)$ & HPBW $\left(^{\circ}\right)$ \\
\hline \hline 75 & 22 & 10 & 23 \\
\hline 77 & 17 & 10.6 & 22 \\
\hline 79 & 8 & 12.6 & 21 \\
\hline 81 & 3 & 12.3 & 20 \\
\hline
\end{tabular}

(b) Difference pattern $\left(A 1: 0.707 \phi 1: 0^{\circ} \& A 2: 0.707 \phi 2: 0^{\circ}\right)$.

\begin{tabular}{|c|c|c|c|}
\hline Operating frequency $(\mathrm{GHz})$ & Beam direction $\left(^{\circ}\right)$ & Sidelobe levels $(d B)$ & HPBW $\left(^{\circ}\right)$ \\
\hline \hline 75 & \pm 18 & 6.6 & 21 \\
\hline 77 & \pm 17 & 14 & 18 \\
\hline 79 & \pm 16 & 7.5 & 17 \\
\hline 81 & \pm 15 & 20.2 & 16 \\
\hline
\end{tabular}

(c) Sum pattern $\left(A 1: 0.707 \phi 1: 0^{\circ} \& A 2: 0.707 \phi 2: 180^{\circ}\right)$.

\begin{tabular}{|c|c|c|c|}
\hline Operating frequency $(\mathrm{GHz})$ & Beam direction $\left(^{\circ}\right)$ & Sidelobe levels $(\mathrm{dB})$ & HPBW $\left(^{\circ}\right)$ \\
\hline \hline 75 & \pm 28 & 12.6 & 23 \\
\hline 77 & 0 & 11.2 & 64 \\
\hline 79 & 0 & 17.9 & 22 \\
\hline 81 & 0 & 13.7 & 20 \\
\hline
\end{tabular}

Table 3.4.: Effect of an operating frequency on an overall pattern $\left(N=5, L_{f}=0.8 \mathrm{~mm}\right)$. 
(a) Fed only by one port $\left(A 1: 1 \phi 1: 0^{\circ} A 2: 0 \phi 2: 0^{\circ}\right)$.

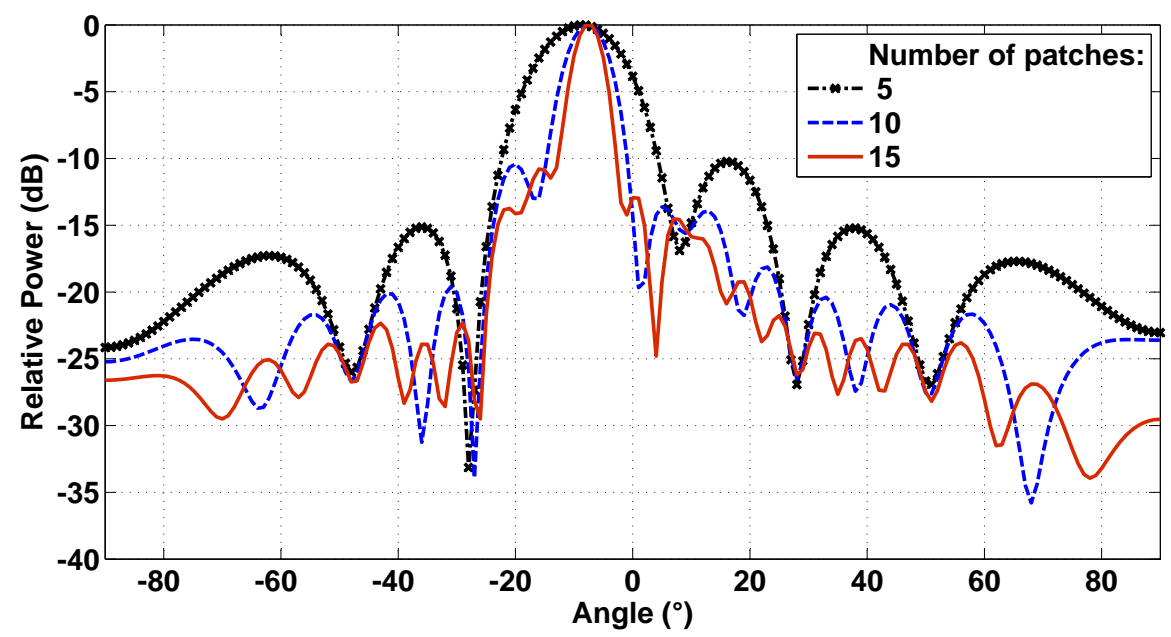

(b) Difference pattern $\left(A 1: 0.707 \phi 1: 0^{\circ} A 2: 0.707 \phi 2: 0^{\circ}\right)$.

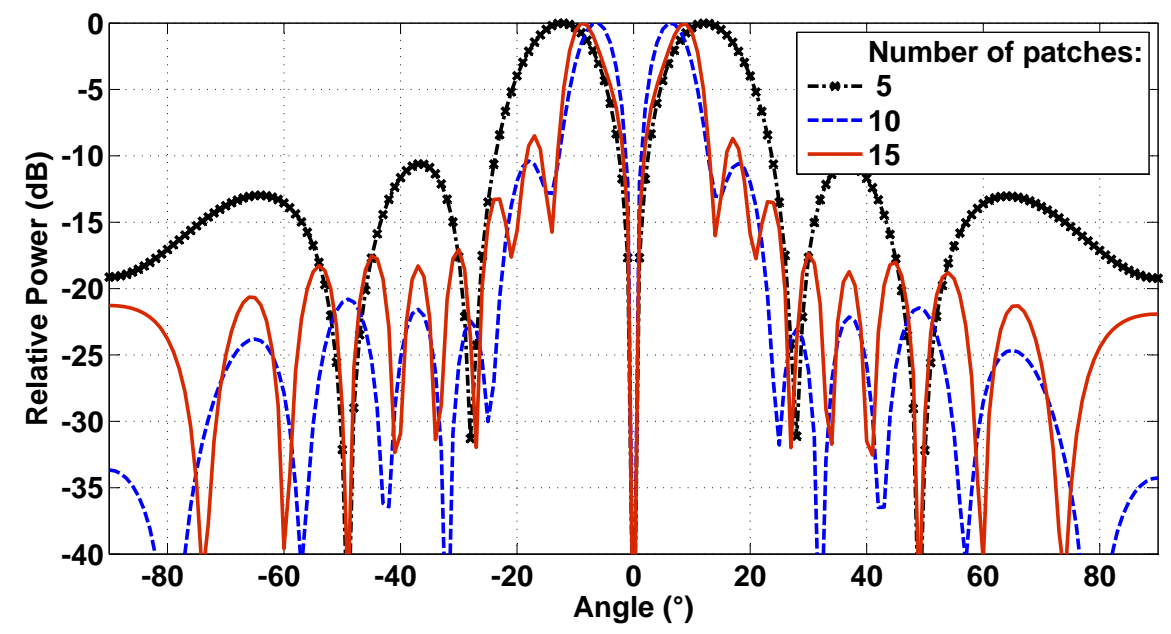

(c) Sum pattern $\left(A 1: 0.707 \phi 1: 0^{\circ} A 2: 0.707 \phi 2: 180^{\circ}\right)$.

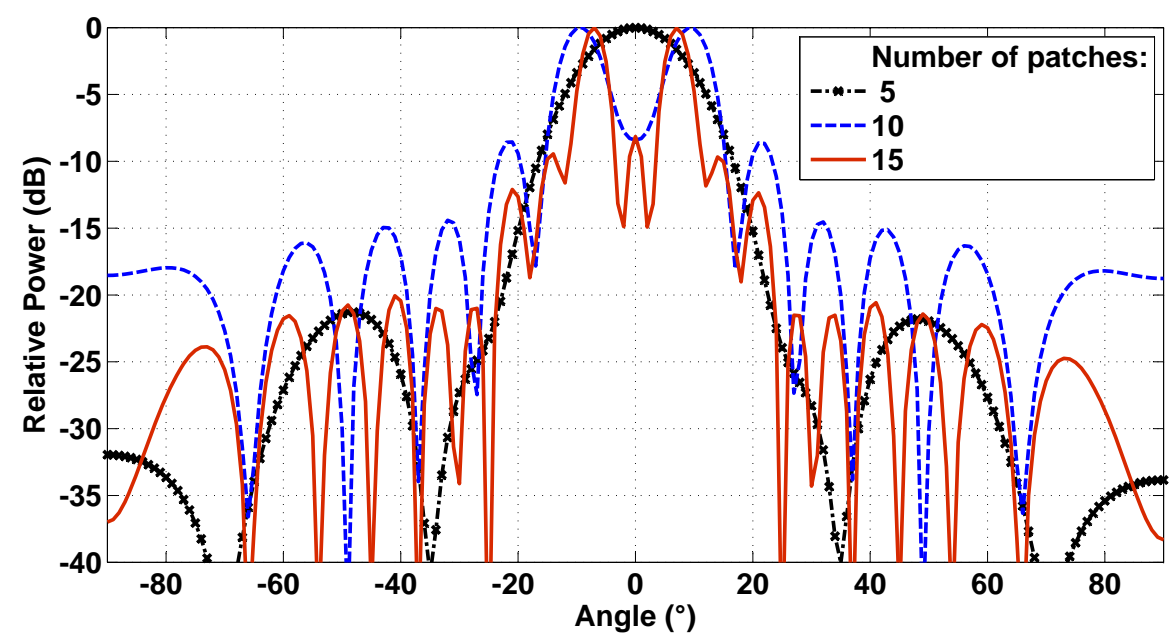

Figure 3.12.: Effect of the number of antenna elements on the radiation pattern of a linear array antenna $\left(L_{f}=1.5 \mathrm{~mm}\right)$. 
(a) Fed only by one port $\left(A 1: 1 \phi 1: 0^{\circ} A 2: 0 \phi 2: 0^{\circ}\right)$.

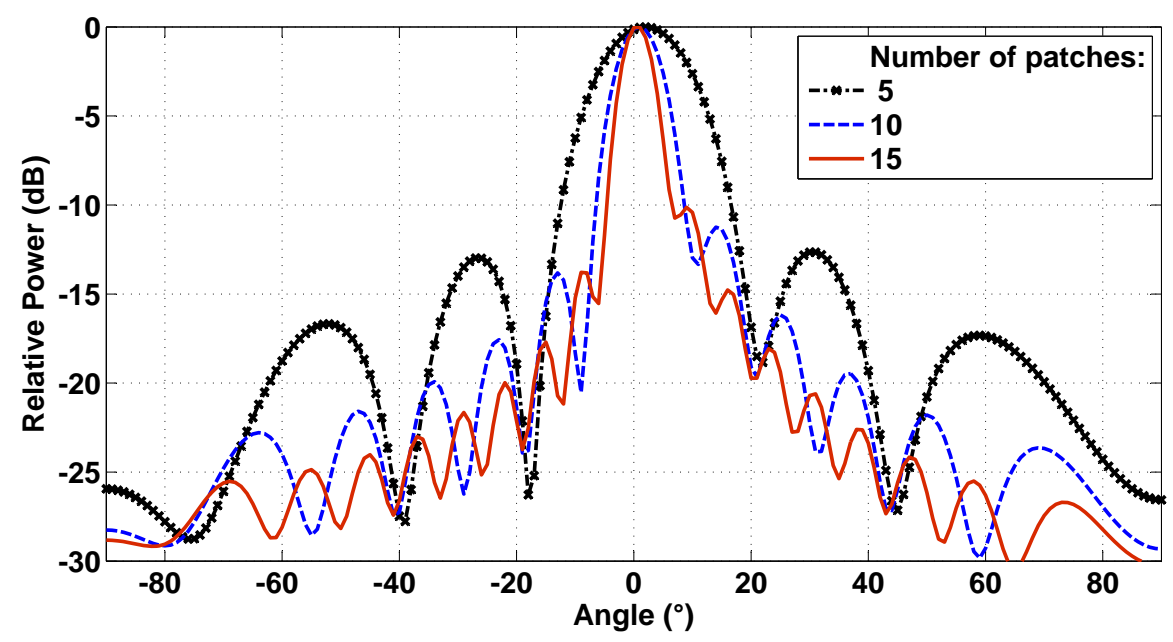

(b) Difference pattern $\left(A 1: 0.707 \phi 1: 0^{\circ} A 2: 0.707 \phi 2: 0^{\circ}\right)$.

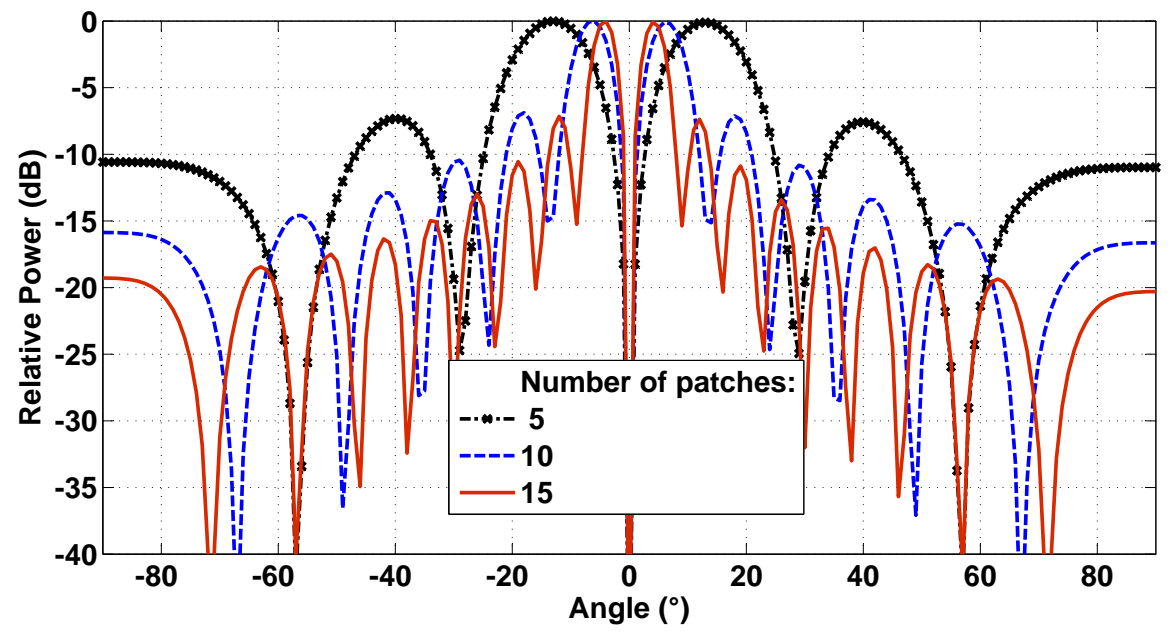

(c) Sum pattern $\left(A 1: 0.707 \phi 1: 0^{\circ} A 2: 0.707 \phi 2: 180^{\circ}\right)$.

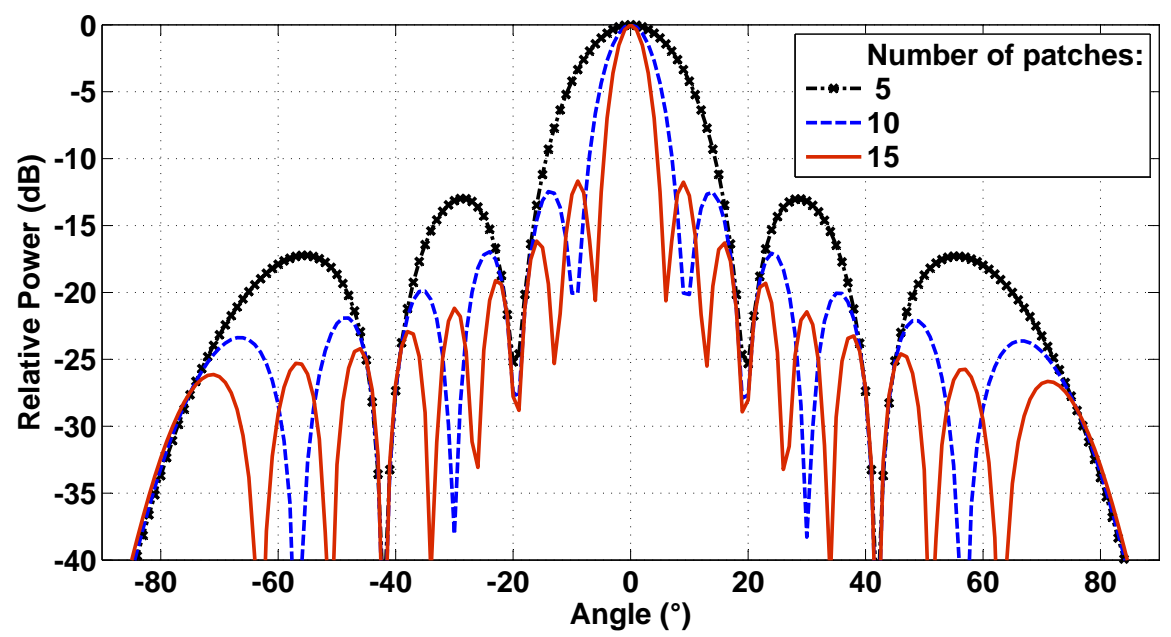

Figure 3.13.: Effect of the number of antenna elements on the radiation pattern of a linear array antenna $\left(L_{f}=1.25 \mathrm{~mm}\right)$. 
(a) Fed only by one port $\left(A 1: 1 \phi 1: 0^{\circ} A 2: 0 \phi 2: 0^{\circ}\right)$.

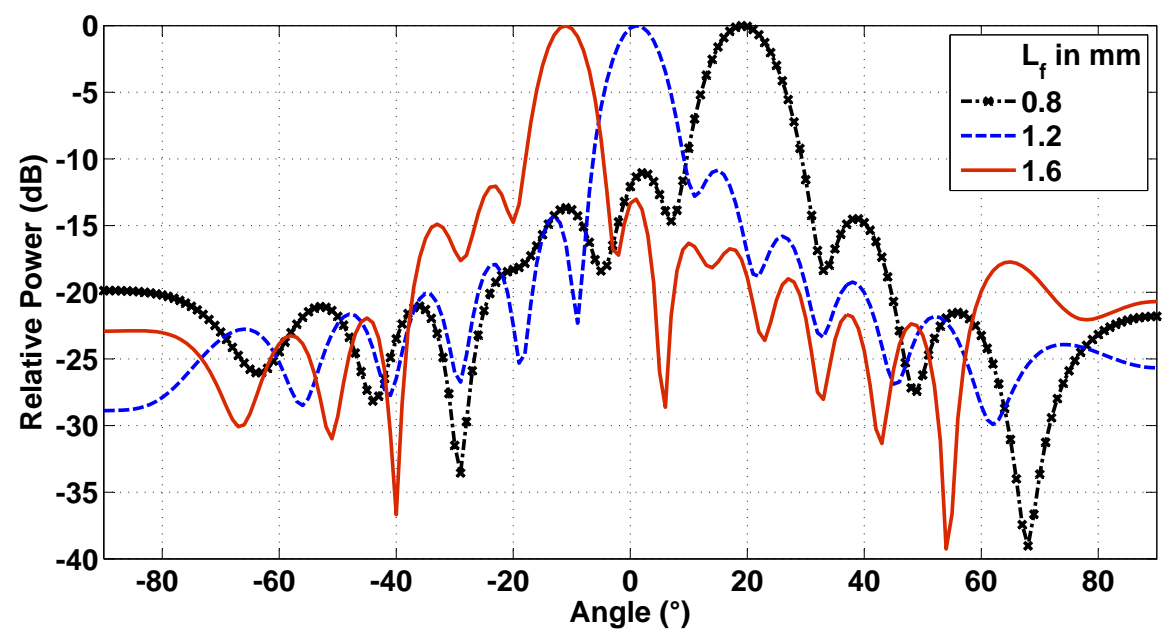

(b) Difference pattern ( $\left.A 1: 0.707 \phi 1: 0^{\circ} A 2: 0.707 \phi 2: 0^{\circ}\right)$.

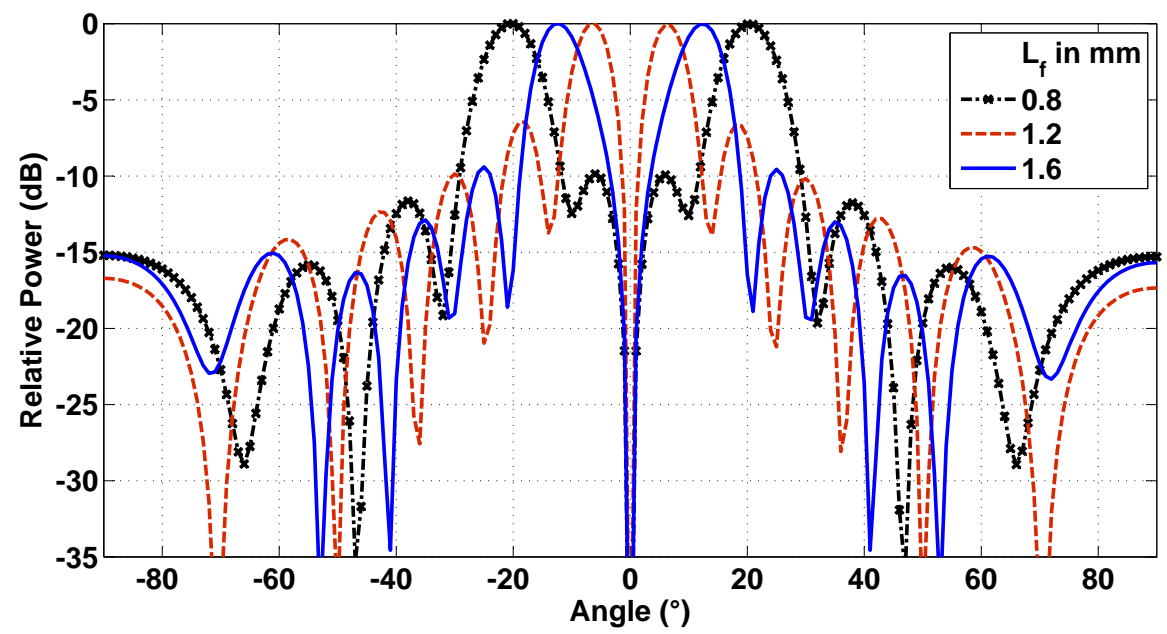

(c) Sum pattern $\left(A 1: 0.707 \phi 1: 0^{\circ} A 2: 0.707 \phi 2: 180^{\circ}\right)$.

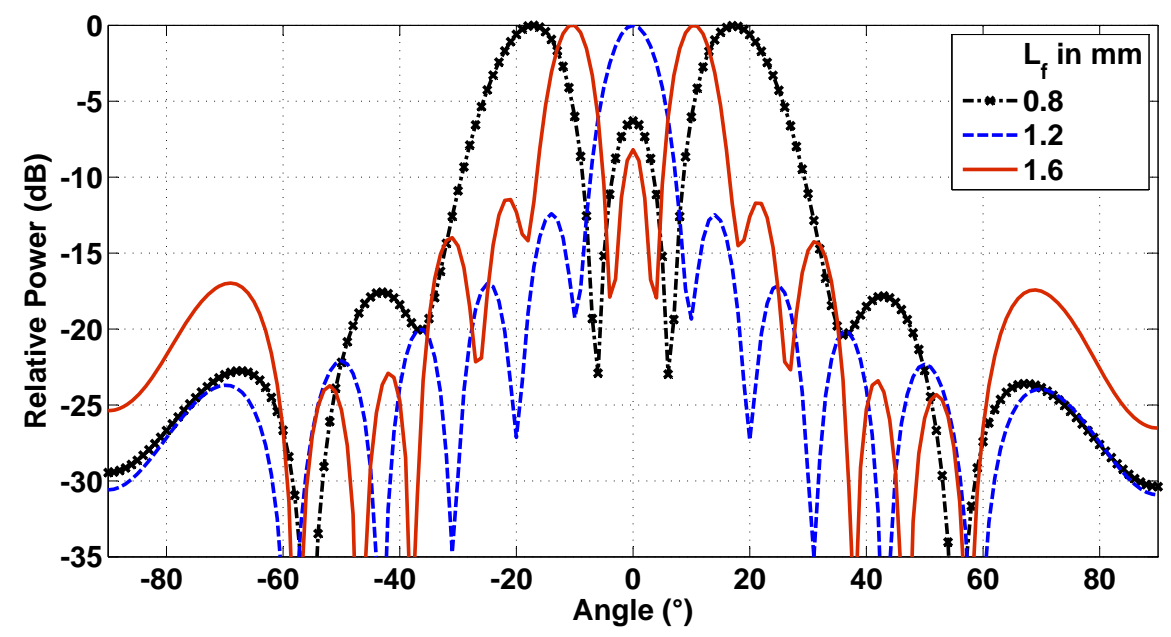

Figure 3.14.: Effect of feed line length on the radiation pattern of a linear array antenna $(N=10)$. 
(a) Fed only by one port ( $\left.A 1: 1 \phi 1: 0^{\circ} A 2: 0 \phi 2: 0^{\circ}\right)$.

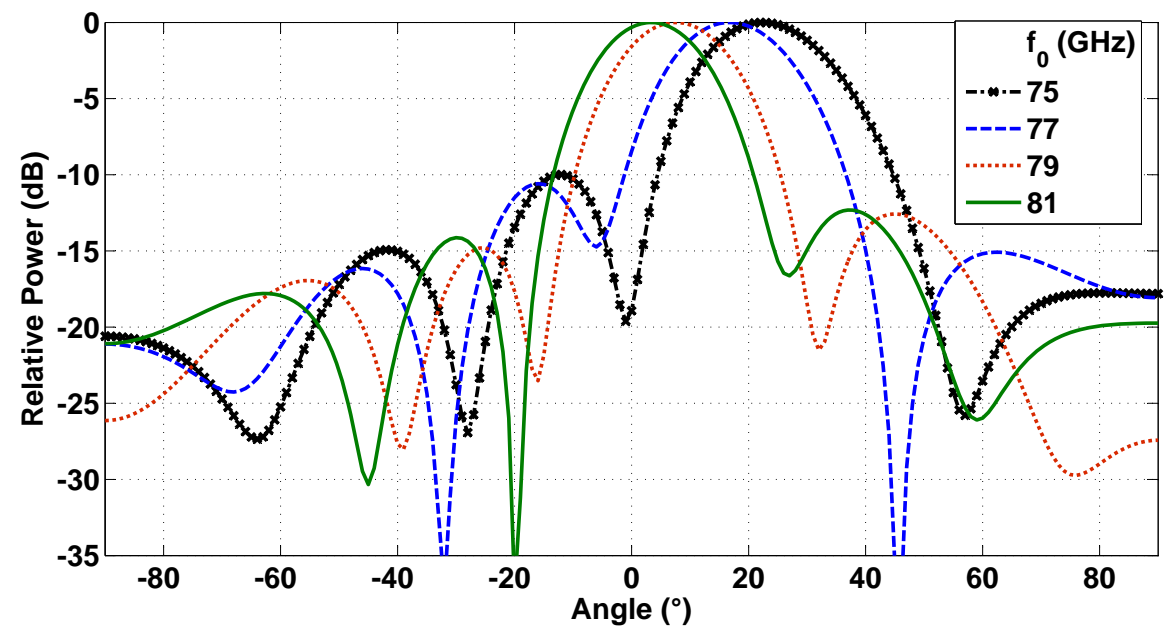

(b) Difference pattern ( $\left.A 1: 0.707 \phi 1: 0^{\circ} A 2: 0.707 \phi 2: 0^{\circ}\right)$.

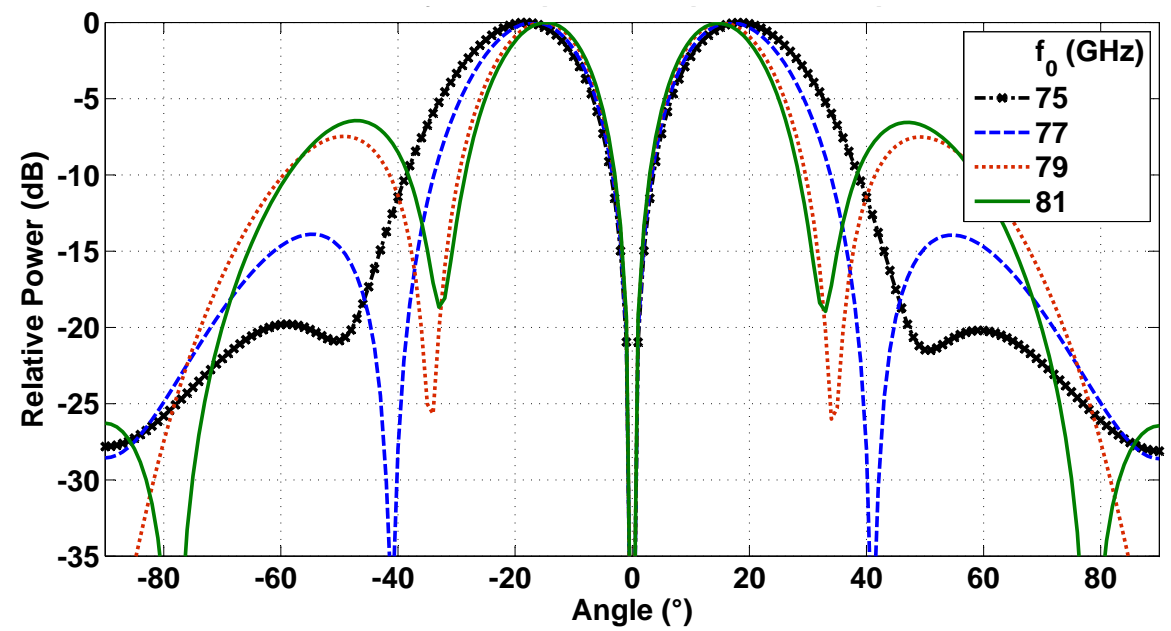

(c) Sum pattern $\left(A 1: 0.707 \phi 1: 0^{\circ} A 2: 0.707 \phi 2: 180^{\circ}\right)$.

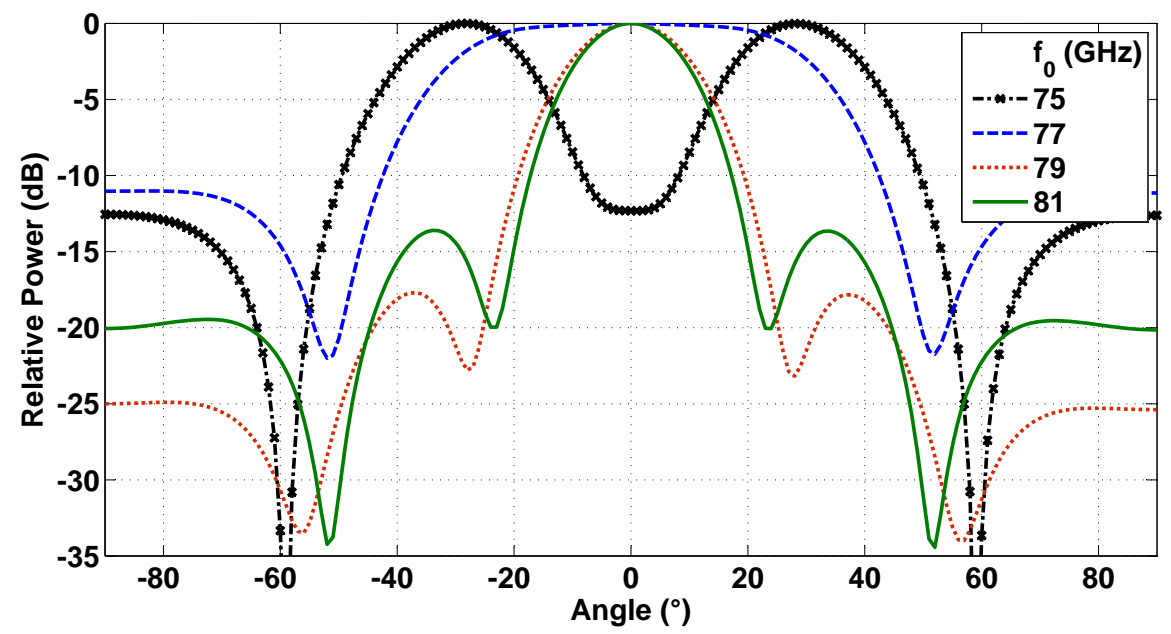

Figure 3.15.: Effect of the operating frequency on the radiation pattern of a linear array antenna $\left(N=5\right.$ and $\left.L_{f}=0.8 \mathrm{~mm}\right)$. 


\section{Measurement Setups}

In this chapter, the mm-wave components that are required for realizing a dual-fed phased array demonstrator at $77 \mathrm{GHz}$ are analyzed and presented in detail. In Section 4.1, two different dual-fed antenna setups are shown, which will be tested in Chapter 5. In Section 4.2, a WR-10 waveguide setup employing a magic-tee, a phase shifter and a variable attenuator is analyzed. In Section 4.3, a $77 \mathrm{GHz}$ transceiver MMIC that houses a vector I-Q modulator in its transmitter path is characterized. Since mm-wave signals are generated by a signal generator with a waveguide output, a waveguide to microstrip line transition is necessary to deliver mm-wave signals to a planar linear array. Hence, a design procedure of a mm-wave waveguide to microstrip line transition is presented in Section 4.4 by aiming at a low-loss and broadband transition. Since the I-Q transceiver MMIC has a fully differential configuration, a planar mm-wave balun is analyzed to convert differential to single-ended signals in Section 4.5 .

\subsection{Overview of measurement setups}

In this section, the system architectures of two different measurement setups are demonstrated. In both setups, the mm-wave signals are generated using a signal generator with a waveguide WR-10 output. These signals are fed into a waveguide magic-tee and are split into two in-phase and equal signals at the output ports [54].

The aim of these setups is to provide the exact control of amplitude ratios $\left(\mathrm{A}_{2} / \mathrm{A}_{1}\right)$ and phase differences $\left(\phi_{2}-\phi_{1}\right)$ between opposite channels (for amplitude ratio $0 \rightarrow 1$ and for phase difference $0^{\circ} \rightarrow 180^{\circ}$ ). 2 different types of phase shifters and attenuators are employed in both setups. As shown in Fig. 4.1(a), the first setup uses a passive WR-10 phase shifter and attenuator, placed into the left waveguide channel. In the right channel, an extra WR-10 attenuator is placed to compensate for the losses due to the asymmetrical waveguide channel setup and errors due to discrete mm-wave components. However, the waveguide phase shifter and variable attenuator must be controlled manually in such a setup causing a tedious and long job during generation of the exact magnitude and phase control of a mm-wave signal. Therefore, a second setup is proposed where an I-Q vector modulator replaces the discrete waveguide phase shifter and variable attenuator. Fig. 4.1(b) shows the second setup which houses an I-Q modulator on an RF board, placed in a transceiver SiGe-MMIC. By means of the vector modulator, amplitude and phase values of mm-wave signals can be controlled easily and simultaneously. More details about the I-Q modulator can be found in Section 4.3. Again, another I-Q modulator is placed in the right channel to avoid any extra unwanted insertion loss even though only one I-Q modulator is adequate to prove the functionality of the pro- 
posed beam steering approach.

(a)

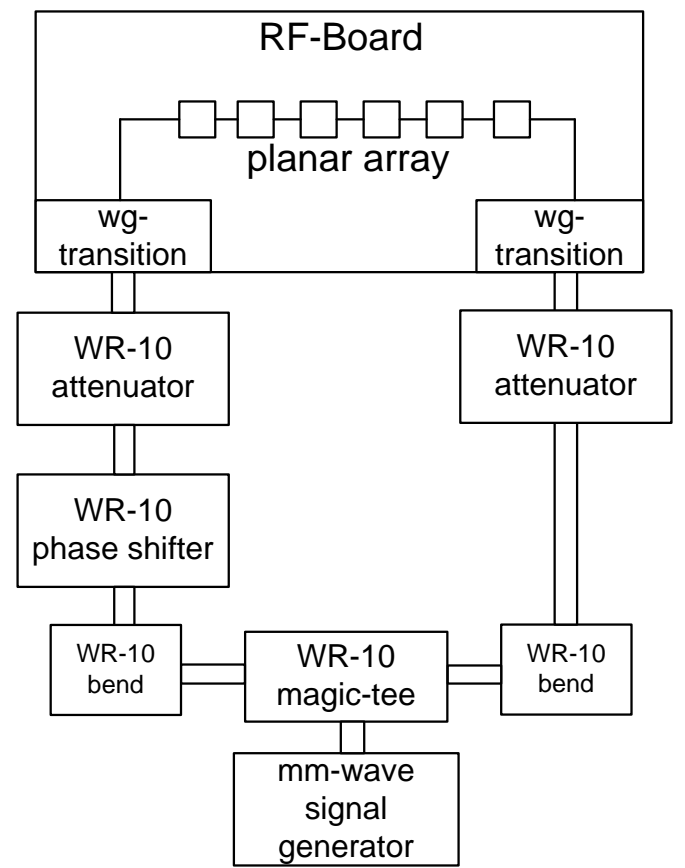

(b)

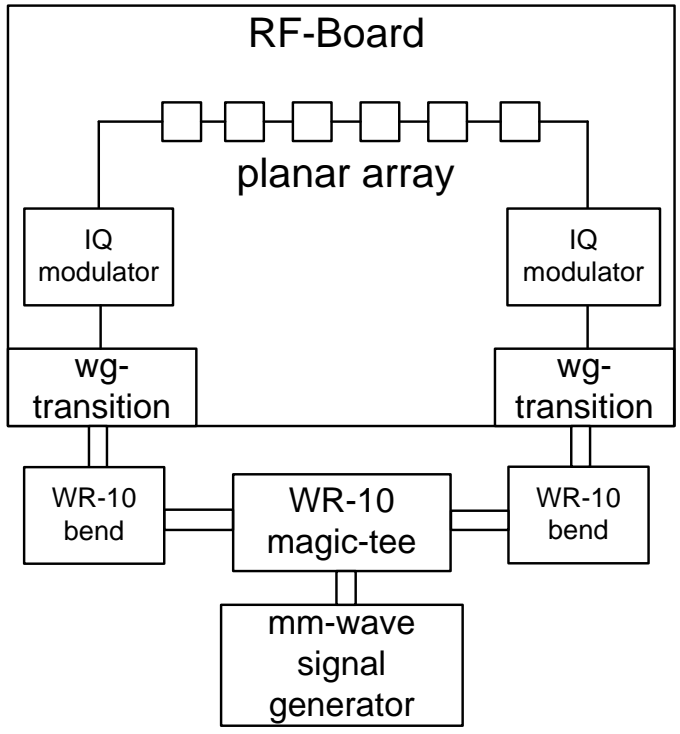

Figure 4.1.: System architectures of two different dual-fed antenna setups: (a) Setup employing passive waveguide phase shifter and variable attenuator, (b) Setup employing an active MMIC I-Q vector modulator.

Waveguide transitions and planar array antennas are common components used in both setups. However, different RF boards have to be manufactured for each setup because the second setup houses an extra bare-die IQ transceiver MMIC which is connected to the rest of the RF circuit via wire bonding.

Fig. 4.2 shows the dual-fed setup employing a waveguide phase shifter and a variable attenuator. In Fig. 4.2(a), a mm-wave signal generator, a magic-tee, a phase shifter and 2 variable attenuators are seen from the top. Fig. 4.2(b) shows the RF board that houses a microstrip array antenna, feed lines and 2 waveguide transitions including waveguide flanges. Waveguide $\mathrm{H}$-bends, used for changing the direction of waveguides, are also seen in the same figure.

Fig. 4.3 shows the second measurement setup employing wire-bonded I-Q transceiver MMICs. In this setup, the waveguide components are used to generate and divide incident signal into two output signals equally (Fig. 4.3(a)). In this setup, the I-Q transceivers are inserted among the waveguide transitions and the planar array antenna by means of mm-wave baluns, as depicted in Fig. 4.3(b). 
(a)

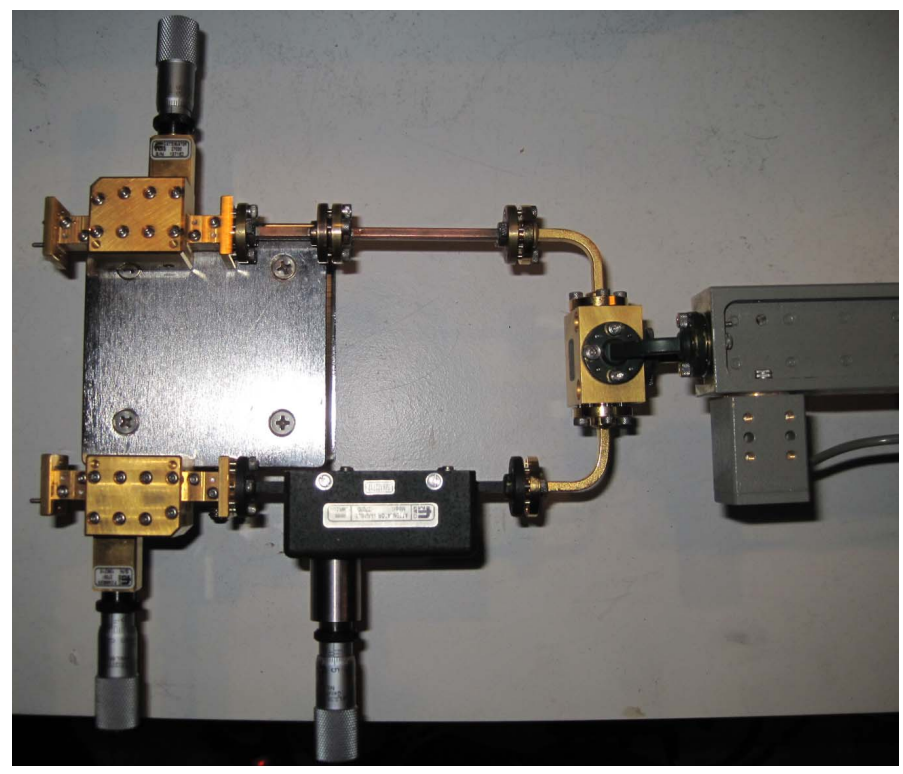

(b)

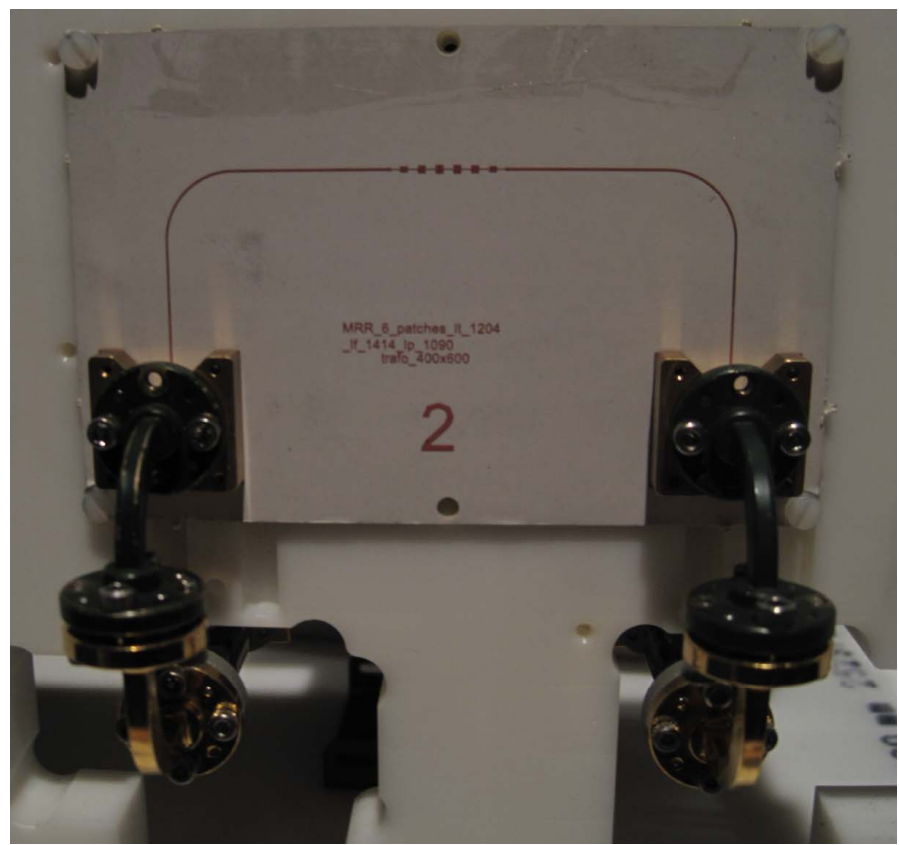

Figure 4.2.: A photograph of the dual-fed setup employing a waveguide phase shifter and variable attenuators: (a) Waveguide components, (b) A planar RF-board (Table A.1). 
(a)

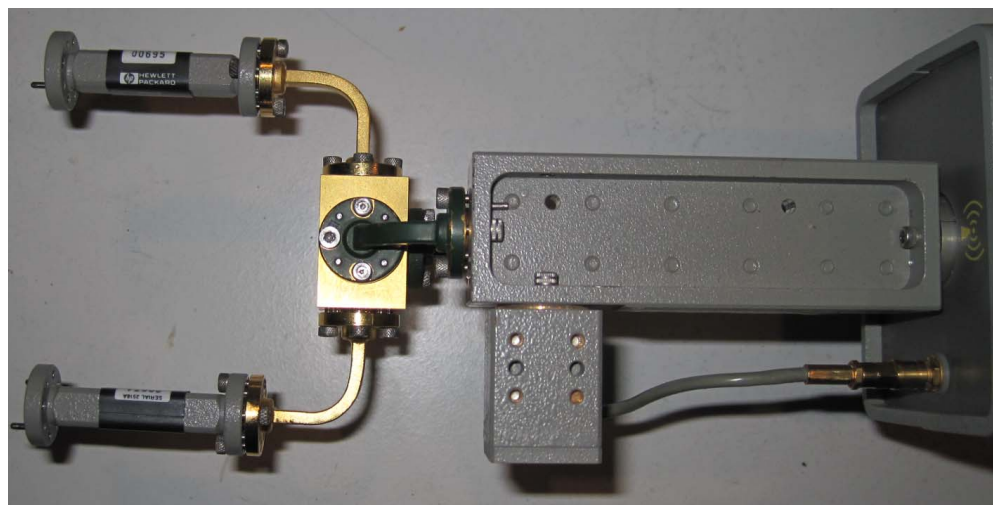

(b)

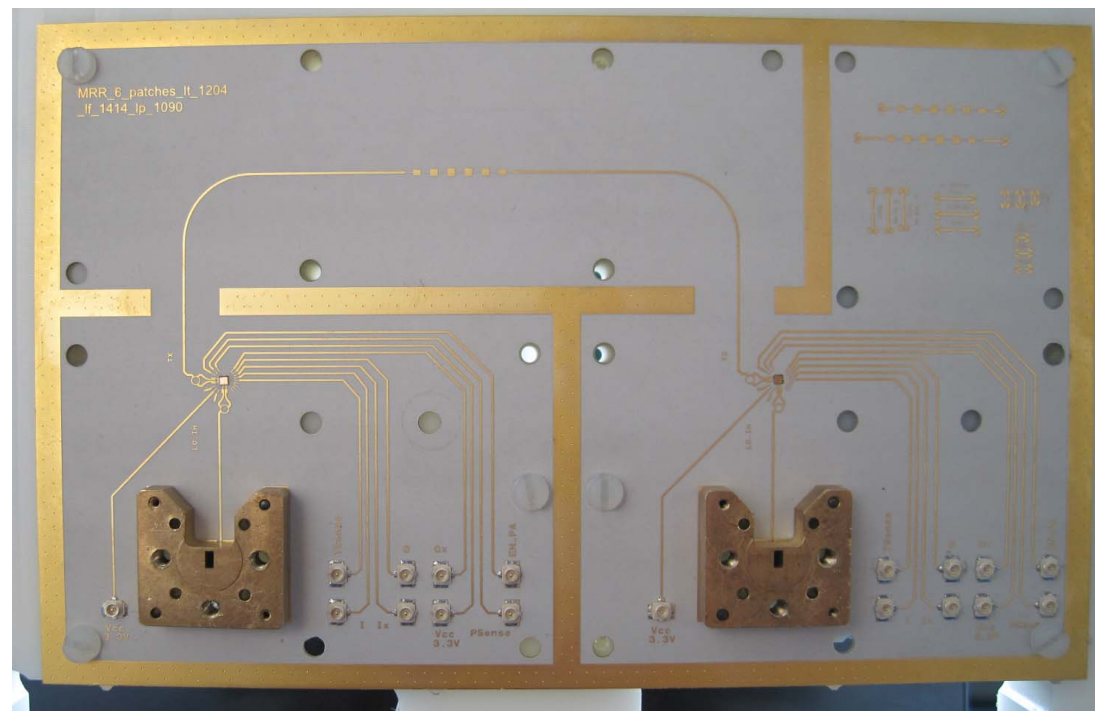

Figure 4.3.: A photograph of the dual-fed setup employing I-Q transceiver MMICs: (a) Waveguide components, (b) A planar RF-board (Table A.1) employing I-Q transceiver MMICs. 


\subsection{Waveguide setup}

In this section, the amplitude ratio and phase difference between the two ports are adjusted to certain values in order to use them for proving the novel phased array approach. Fig. 4.4 shows a block diagram of the characterized waveguide setup. The setup consists of a magictee, two variable attenuators and a phase shifter. The magic-tee is used for dividing the input signal equally into two in-phase output signals [54]. The phase shifter has a micrometer drive permitting change of phase up to $210^{\circ}$ [55]. The change in phase causes almost no change in amplitude value (maximum $0.2 \mathrm{~dB}$ within the frequency of interest). Variable attenuators also have micrometer drives permitting change of attenuation value up to $35 \mathrm{~dB}$ [56]. In the same way as the phase shifter, the change in amplitude also leads to change in insertion phase. Therefore, after characterization of the waveguide setup, a look-up table is generated for different amplitude ratios and phase differences by taking these changes into account.

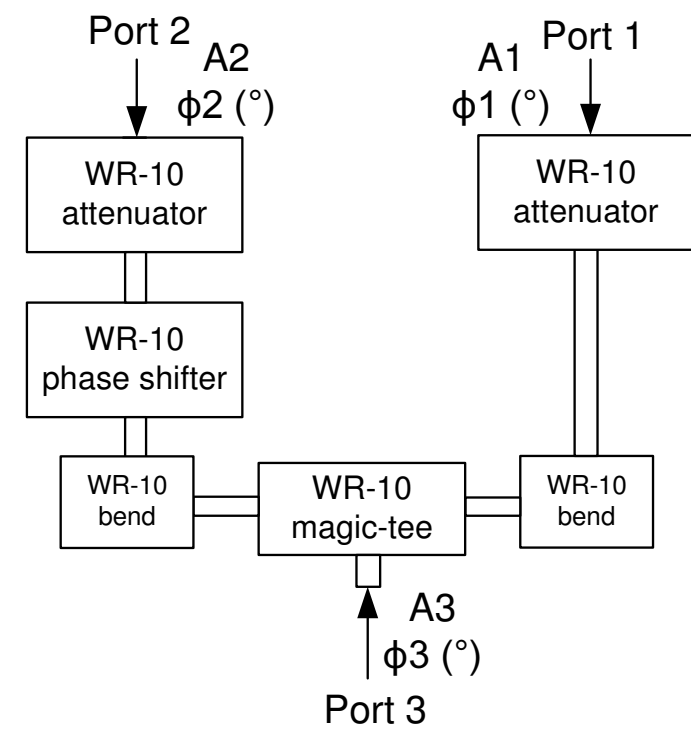

Figure 4.4.: The waveguide setup used for characterization.

Measurements are performed using a 2 port mm-wave vector network analyzer. For each measurement, the third port is terminated with a $50 \Omega$ matched load. After optimizing micrometer drives of the phase shifter and 2 variable attenuators manually, a look-up table is generated for some specific amplitude ratios and phase differences. This means, for each amplitude or phase ratio, values of 3 different micrometer drives are recorded. These values will be later used for beam steering in Chapter 5 .

After the generation of two different look-up tables for the amplitude ratios and phase differences between two channels, the amplitude ratios $\left(\mathrm{A}_{2} / \mathrm{A}_{1}\right.$ in logarithmic scale) are shown in Fig. 4.5. These ratios, given in logarithmic scale, are almost constant throughout the frequency band from 75 to $80 \mathrm{GHz}$. Fig. 4.6 shows phase differences between two output ports 
$\left(\phi_{2}-\phi_{1}\right)$ in degrees) up to $200^{\circ}$ with $20^{\circ}$ steps. The phase values for each step are also constant between 75 and $80 \mathrm{GHz}$, like in Fig. 4.5.

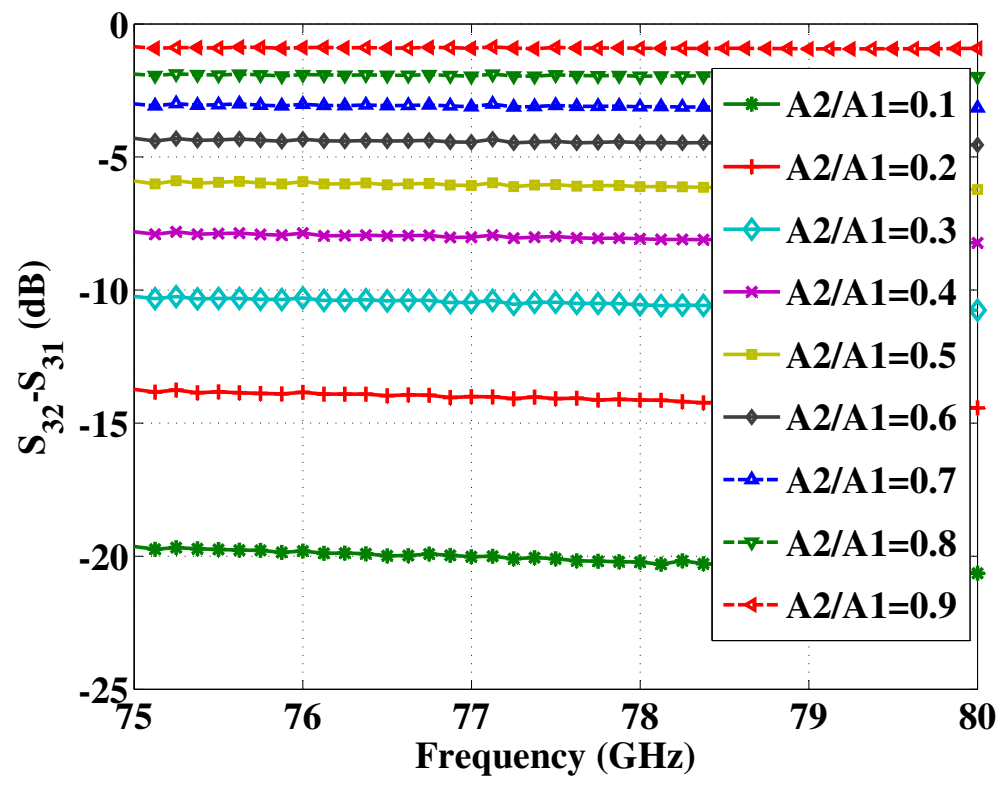

Figure 4.5.: Amplitude ratios between both channels by adjusting the variable attenuators.

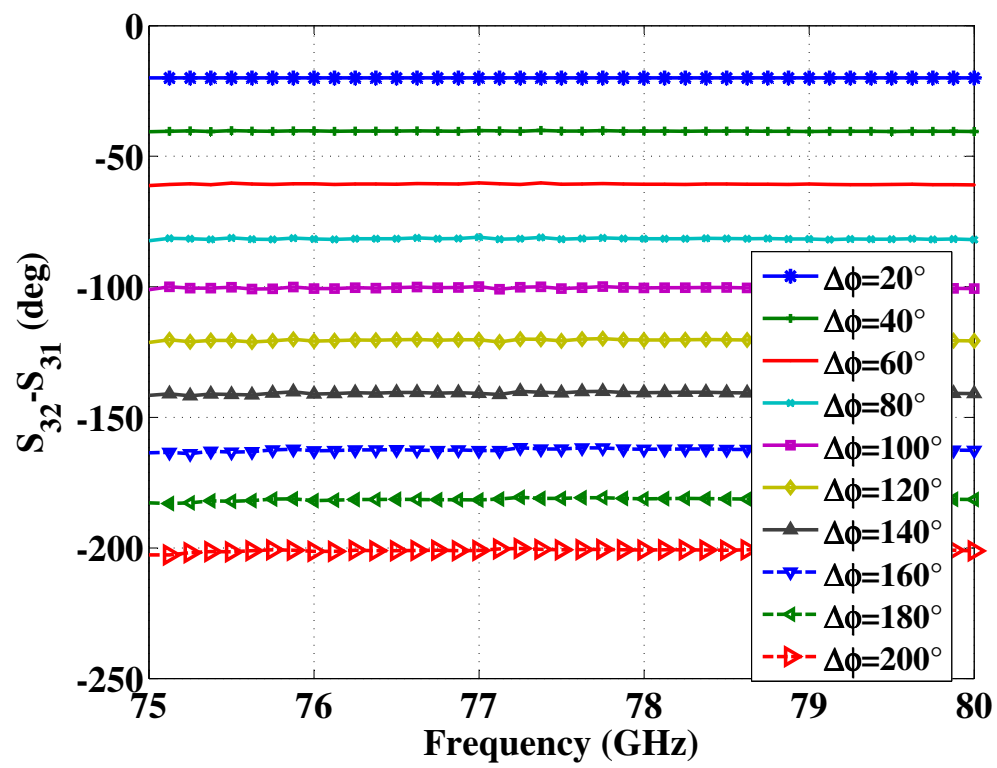

Figure 4.6.: Phase differences between both channels by adjusting the phase shifter at Port 2 . 


\subsection{A 77-GHz SiGe MMIC I-Q vector modulator}

In this section, a $77 \mathrm{GHz}$ MMIC I-Q transceiver module based on a Silicon-Germanium HBT technology is characterized. This module has been designed and used for a $77-\mathrm{GHz}$ phased-array radar transmitter in [57]. Since it consists of a fully differential active I-Q vector modulator in its transmitter path [9], this transceiver will be used as a phase shifter and variable attenuator for one of the measurement setups, shown in Section 4.1. Since only the I-Q modulator is of interest, only the transmitter path of the transceiver is characterized in this section. A detailed block diagram of this transceiver and the working principle of the I-Q modulator are presented in Section 4.3.1. A complete characterization setup including $\mathrm{RF}$ and control boards are shown in Section 4.3.2. Finally, measurement results of the I-Q modulator are presented in Section 4.3.3.

\subsubsection{Architecture}

Fig. 4.7(a) shows building blocks of a fully integrated I-Q transceiver module. A mm-wave signal is supplied to a differential LO input by an external mm-wave source. Then, the signal is divided into two equal signals by means of an active power splitter. One of them is delivered to LO output for the daisy-chaining option. The other one is delivered to quadrature LO generation component to distribute LO signal not only to I-Q vector modulator but also to the IQ mixers. The I-Q vector modulator is used as a phase shifter and variable attenuator simultaneously. In order to deliver sufficient output power to the antenna port, a medium power amplifier is used. This amplifier can be turned on/off via an enable control unit (EN). This property may be used for beamforming applications by turning on/off the channel. Since the receiver and transmitter channels use a common antenna port (monostatic antenna), a differential rat-race coupler is used to separate transmitter and receiver signals. Since only transmitter path is within the scope of the dissertation, the receiver path will not be covered in this work.

Fig. 4.7(b) exhibits the chip micrograph showing locations of each individual component and differential input/output ports. Locations of differential ports have been optimized for easy daisy-chaining. With this option, multiple chips can be cascadable in series so that it can be used for multi-channel beamforming applications. The total die size of the chip is $1028 \mathrm{um}$ x $1128 \mathrm{um}$. Total power consumption of the complete transceiver is $320 \mathrm{~mW}$ (from a single $3.3 \mathrm{~V}$ voltage supply). In order to track power and temperature values continuously, on-chip temperature and power sensors are included.

Instead of using a phase shifter and variable gain amplifier separately, an I-Q vector modulator is used for the measurement setup which controls both amplitude and phase values of the signal simultaneously. An arbitrary signal can be described as a vector signal in a polar coordinate system with its amplitude and phase parameters. This signal can also be defined as a sum of two signals in a rectangular coordinate system with coordinates of 'I' and 'Q', one in-phase and the other one quadrature (Fig. 4.8). By adjusting amplitudes and polarities 
(a) Block diagram.

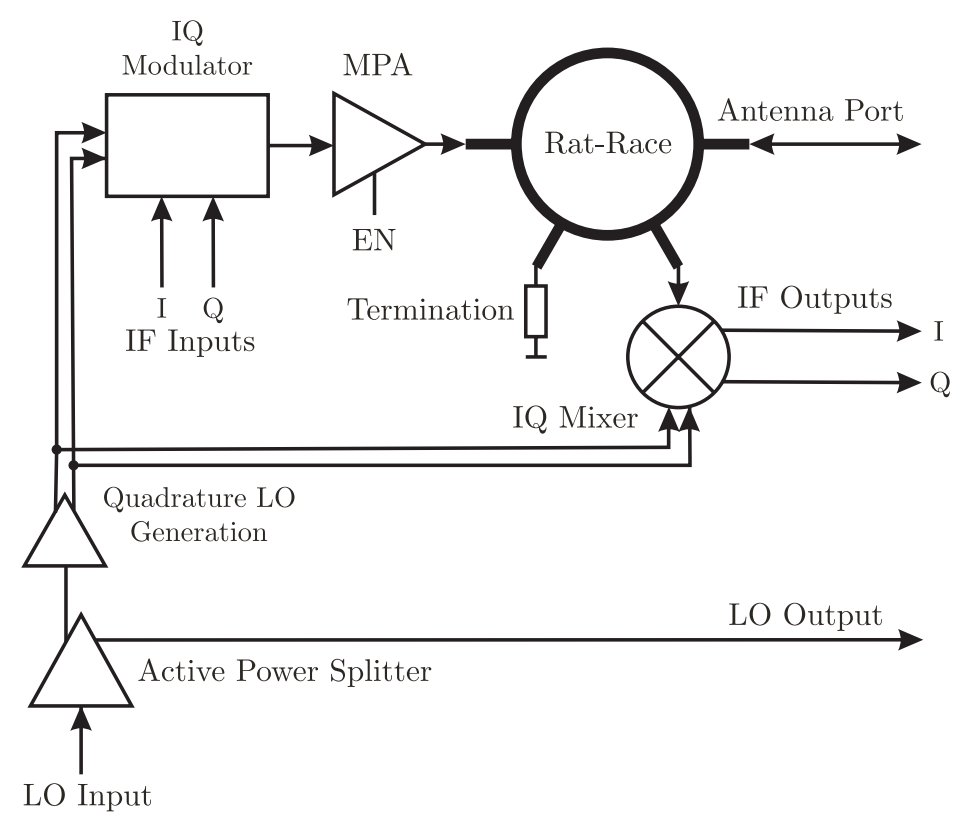

(b) Chip microphotograph.

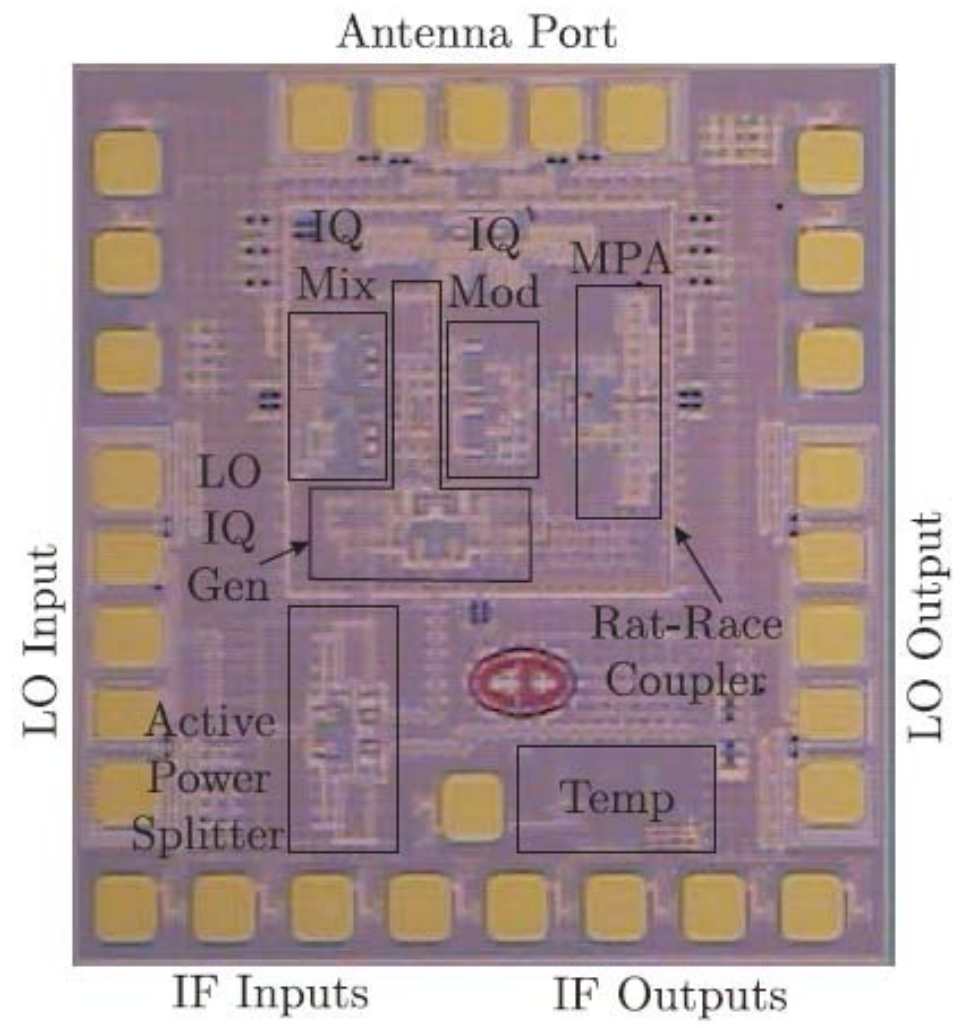

Figure 4.7.: An I-Q transceiver chip. 
of I and Q input signals, different desired phase and amplitude responses can be obtained.

[58], [59] report mm-wave MMIC I-Q vector modulators and measurement setups for the characterization.

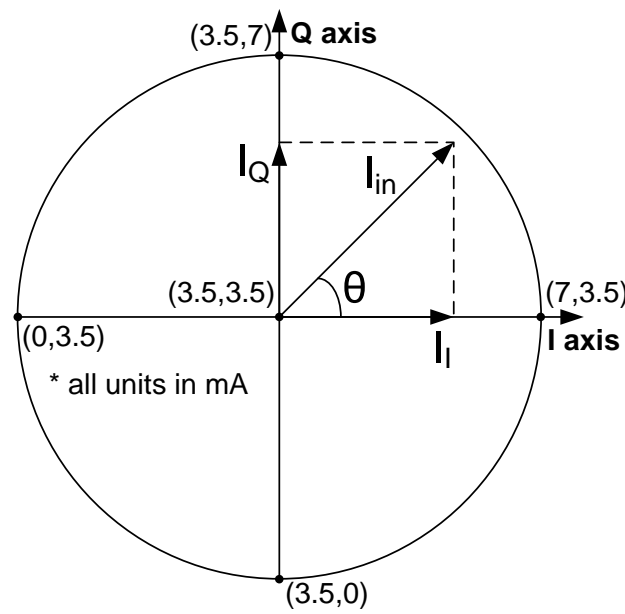

Figure 4.8.: An I-Q vector model.

$$
\begin{gathered}
I_{I}(m A)=3.5+I_{i n} \cos \theta \\
I_{I_{x}}(m A)=3.5-I_{i n} \cos \theta \\
I_{Q}(m A)=3.5+I_{i n} \sin \theta \\
I_{Q_{x}}(m A)=3.5-I_{\text {in }} \sin \theta
\end{gathered}
$$

Eq. 4.1 - 4.4 show the relation of input baseband signals and output mm-wave signal where $I$ and $I_{x}$ are differential baseband input signals of the in-phase modulator, while $Q$ and $Q_{x}$ are differential baseband input signals of the quadrature-phase modulator. By supplying these 4 current signals to the vector modulator, amplitude $\left(I_{i n}\right)$ and phase $(\theta)$ values of the mm-wave signal can be controlled.

\subsubsection{Test setup}

In Fig. 4.9, a block diagram of the test setup is shown. The aim of the test setup design is to control amplitude and phase values of transmitter signals via the IF inputs of the I-Q modulator. Then these values are recorded into a look-up table in order to use them for a beamforming application. Phase and amplitude errors are corrected using the look-up table. IF input signals of the I-Q modulator are generated via data acquisition card from National 
Instruments [60]. Since the existing DAQ card cannot generate adequate current signals, voltage signals are converted to current signals using a control board. These current signals are delivered to the I-Q transceiver chip, wire-bonded on the RF test board. Here, the Sparameters are measured via on-wafer probing by a 2-port mm-wave vector network analyzer with WR-10 waveguide ports. W-band probes by GGB Industries are used to make on-wafer measurements. Afterwards, the measured S-parameter results (in addition to the amplitude values of IF control signals) are saved over GPIB on a PC using LabVIEW.

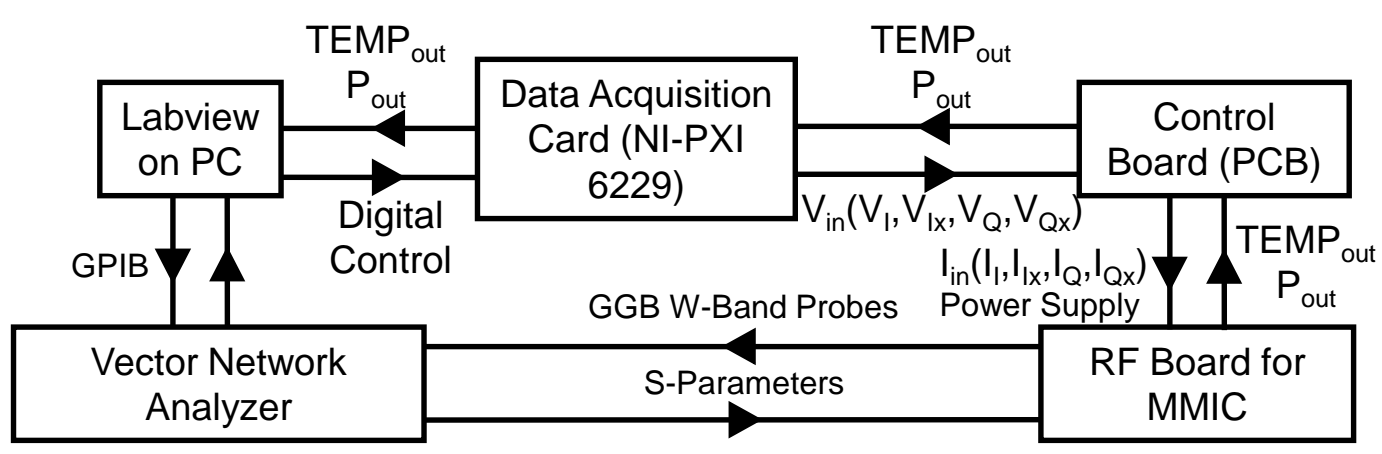

Figure 4.9.: The block diagram of the measurement system for the I-Q transceiver.

Two test boards were designed and manufactured for the characterization of the I-Q modulator. These are a control board used for regulating baseband signals and an RF board housing the I-Q modulator.

Control board: In order to control and characterize the I-Q transceiver module, a control board has been manufactured on FR-4 (Fig. 4.10). Fig. 4.10(a) depicts the main building blocks of the board. It is used as an interface board between RF board and DAQ card used for generating and acquiring analog signals. It supplies the required power for the complete circuit on the PCB and RF board that houses the I-Q transceiver MMIC. It acquires analog signals from the M-Series NI-DAQ card (a high speed data acquisition card from National Instruments). Since the I-Q vector modulator is controlled via 4 current IF input signals and the existing card cannot deliver required current value, a voltage to current converter has been designed on the control board. This converter was designed using a single op-amp and a pnp transistor. After the generation of 4 analog signals with different phases and amplitudes at NI-DAQ card, they are filtered by an analog low pass filter to filter out unwanted signals that may be present in a band above the desired frequency band. Then, these filtered voltage signals are converted into current signals via voltage to current converters. These signals are supplied to the IF inputs of the I-Q modulator in the RF board. Fig. 4.10(b) shows the manufactured control board including its connectors.

$\boldsymbol{R F}$ test board: Since the I-Q transceiver is designed with differential ports and our measurement equipments are equipped with single-ended ports at $77 \mathrm{GHz}$ frequency range, measurements had to be performed on an RF-board by converting differential signals to single-ended 
(a) Block diagram.

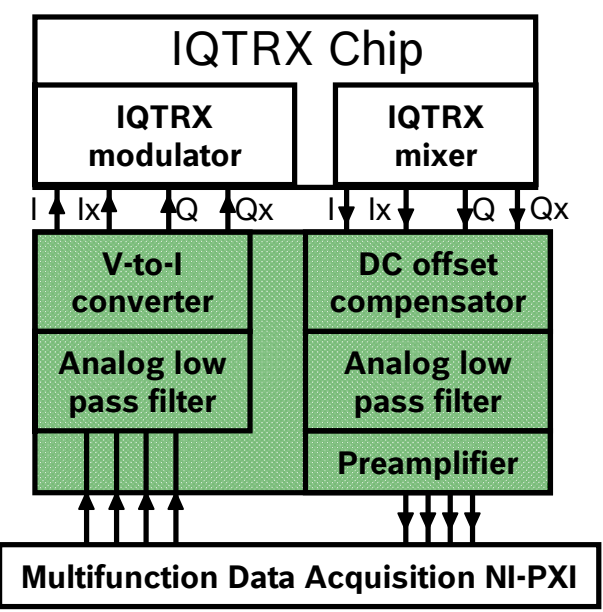

(b) Manufactured PCB.

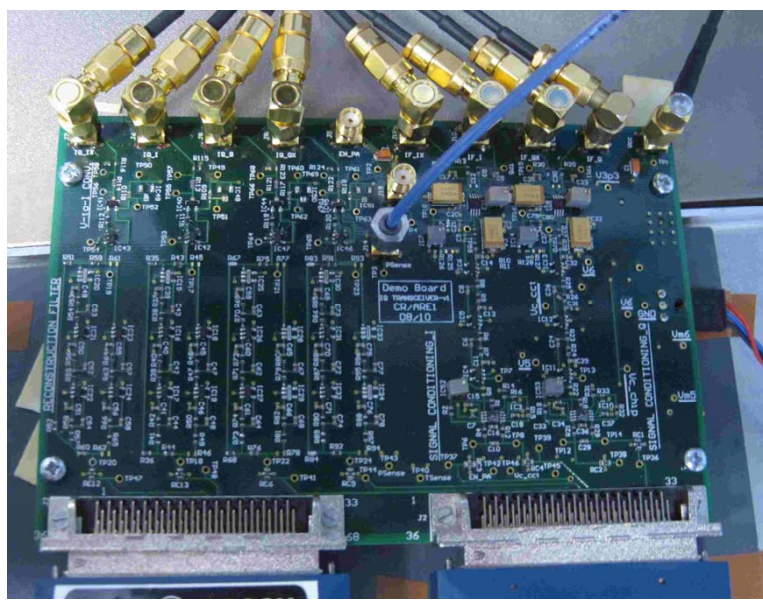

Figure 4.10.: A control board for the I-Q transceiver.

ones via baluns on the board. Therefore, an RF test board was designed and fabricated on a TSM-30 dielectric substrate from Taconic with a thickness of 127 um [61]. The bare-die transceiver MMIC was bonded into a cavity with silver epoxy in the board. The RF board consists of the wire bonded I-Q transceiver chip, bond compensation circuits, mm-wave planar rat-race baluns and coplanar probes for each port (Fig. 4.11(b)). The insertion losses due to baluns, bond compensation circuits and wire bonds are not de-embedded from the measurements. On-wafer measurements are performed on this board at the $77 \mathrm{GHz}$ frequency range, as shown in Fig. 4.11(a). The measurements are carried out with the vector network analyzer Anritsu 37397D connected to the W-band probes.

(a) Manufactured PCB.

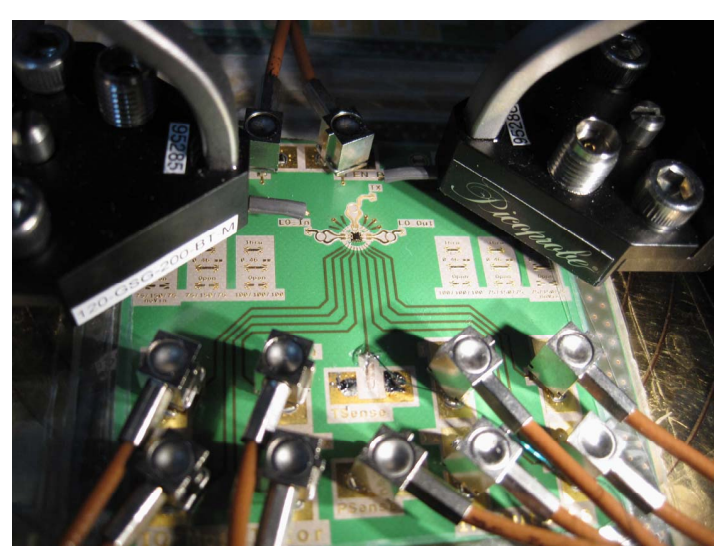

(b) Baluns and bonded chip.

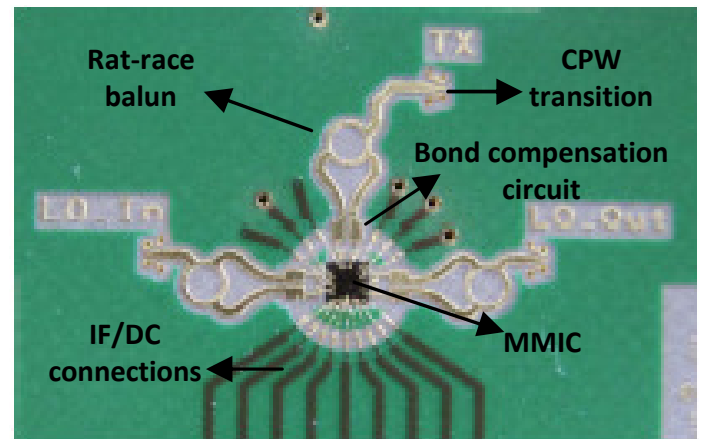

Figure 4.11.: RF test board for the I-Q transceiver. 


\subsubsection{Measurement results}

The forward transmission characteristics $\left(S_{21}(\mathrm{~dB})\right.$ and $\left.S_{21}(\mathrm{deg})\right)$ in the frequency band 75 to $81 \mathrm{GHz}$ are plotted for different amplitude and phase states, where the control input currents of $I, I_{x}, Q$ and $Q_{x}$ have been swept from $0 \mathrm{~mA}$ to $7 \mathrm{~mA}$ with discrete steps. The common mode current level for the input currents is $3.5 \mathrm{~mA}$. Fig. 4.12 shows a complete setup during on-wafer characterization. Fig. 4.13 shows the transmission characteristics for the different phase states. As seen in Fig. 4.13(a), the I-Q transceiver provides a continuous phase shift of 0 to 360 degrees from 75 to $81 \mathrm{GHz}$, with almost consistent conversion gain $(\sim 6.5 \mathrm{~dB})$ with respect to the phase shift and the frequency change, shown in Fig. 4.13(b). The amplitude imbalance is within $1 \mathrm{~dB}$ at $77 \mathrm{GHz}$. The measured input and output reflections are less than $-8 \mathrm{~dB}$ at $77 \mathrm{GHz}$.

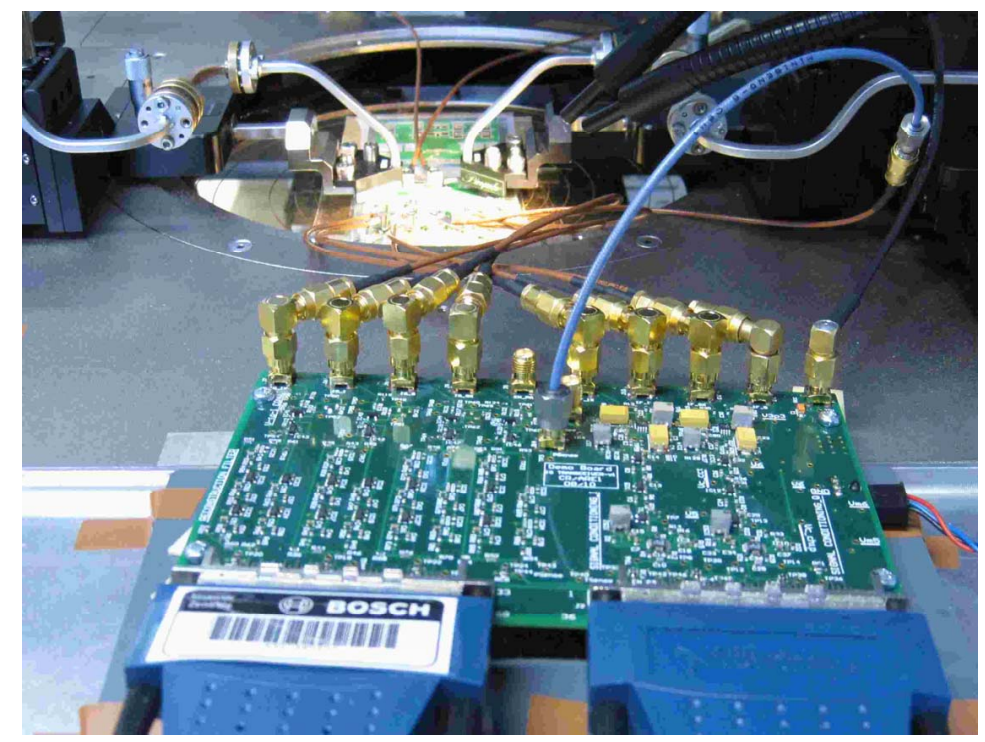

Figure 4.12.: Measurement setup for the I-Q transceiver module.

In Fig. 4.14, different amplitude states are plotted. By adjusting the control IF current signals, conversion gain can be varied up to $20 \mathrm{~dB}$, as plotted in Fig. 4.14. The phase imbalance is within $15^{\circ}$ between $75 \mathrm{GHz}$ to $81 \mathrm{GHz}$. 
(a) Phase values of the I-Q modulator versus control current.

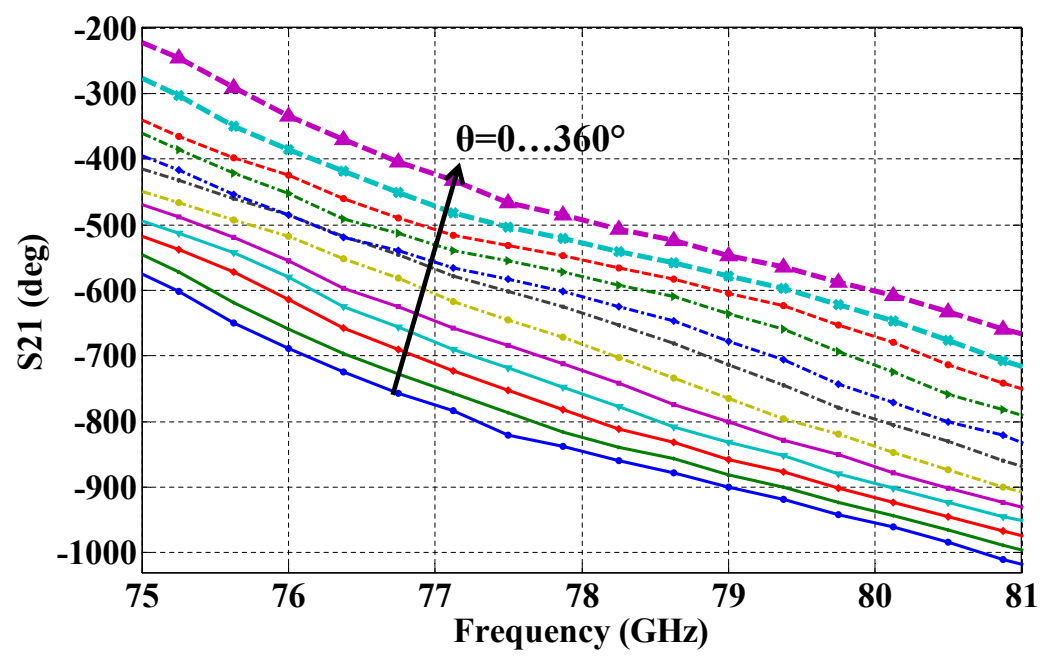

(b) Amplitude values of the I-Q modulator versus control current.

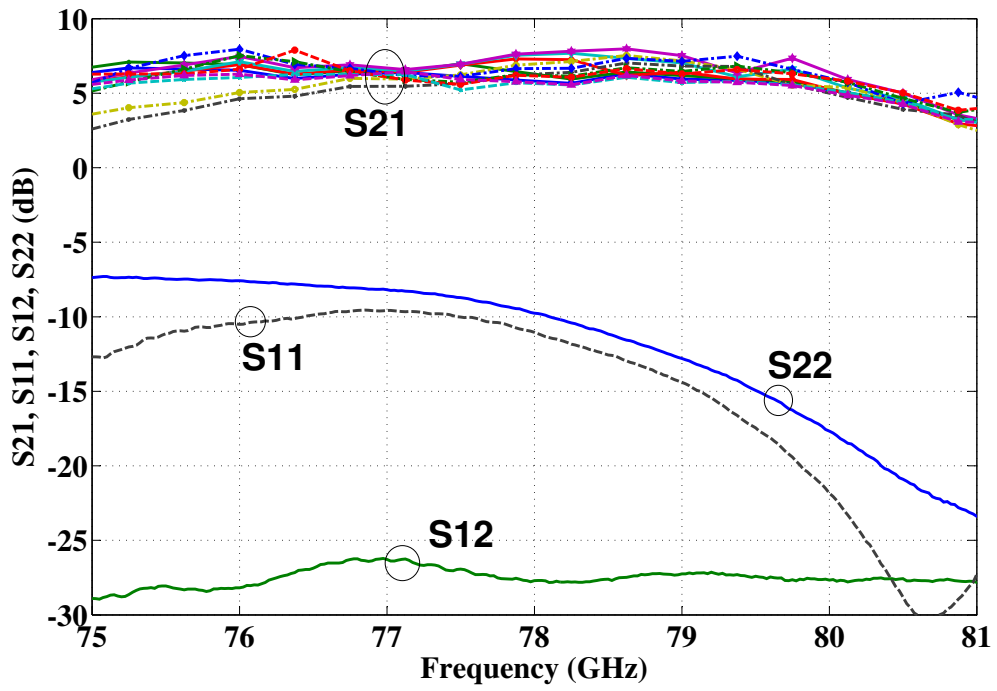

Figure 4.13.: Measured transmission characteristics for different phase states. The curve parameter is the phase of the current $\theta=\arctan ((Q-3.5) /(I-3.5))$, while the absolute current $I_{\text {tot }}=\sqrt{(I-3.5)^{2}+(Q-3.5)^{2}}=3.5 \mathrm{~mA}$. 


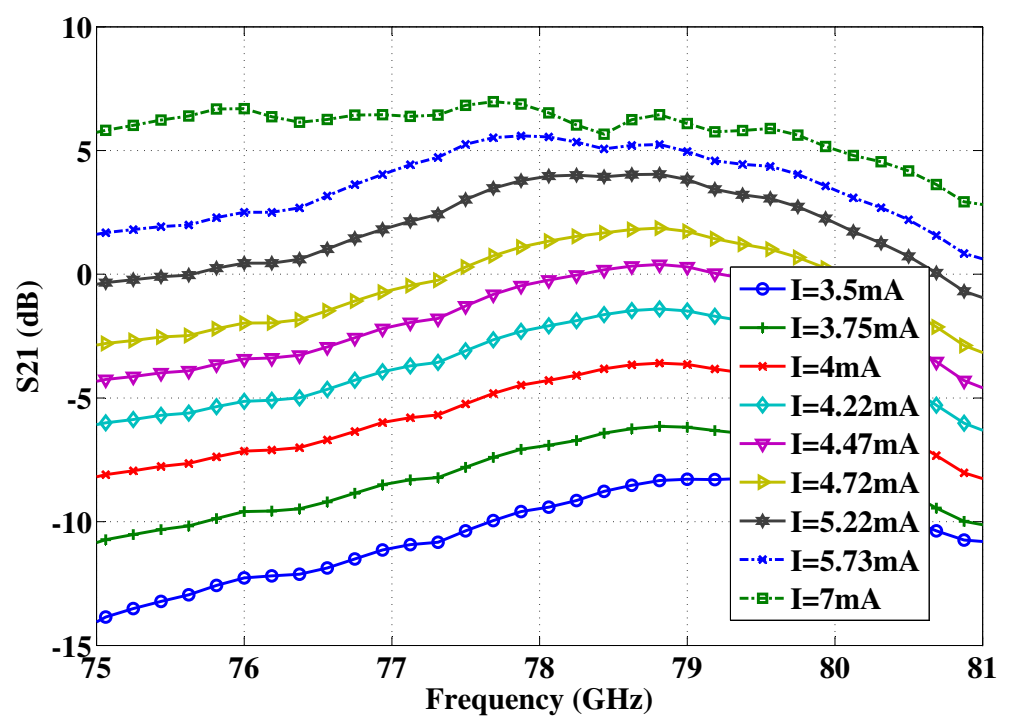

Figure 4.14.: Measured transmission characteristics for different amplitude states. The curve parameter is the current in the I-channel $I$, the current in the Q-channel $Q=3.5$ $\mathrm{mA}$.

\subsection{A millimeter-wave waveguide to microstrip line transition}

Based on the planned test setup for the dual-fed phased array, some mm-wave components have waveguide inputs and outputs whereas some of them have planar inputs and outputs. Linear array antennas and bare-die IQ transceiver MMIC have been designed and characterized using microstrip line technology. However, mm-wave signals at $77 \mathrm{GHz}$ range are generated via a mm-wave signal generator which has a WR-10 waveguide output and Wband commercial waveguide phase shifter and attenuator have WR-10 inputs and outputs. In order to connect these components to each other at this frequency band, a waveguide to microstrip line transition is necessary. Hence, a new millimeter-wave rectangular waveguide to microstrip line transition is designed and presented in this section.

This section presents a compact topside waveguide to microstrip line transition design in the 76-81 GHz frequency band. The transition is fabricated on a grounded single layer substrate using a standard PCB fabrication process. A microstrip patch antenna and a novel edge feed technique are used for the impedance transformation. In the first step of the design, a simple conventional WR-10 waveguide is employed (Type A). In the second step of the design, a WR-10 waveguide with an inductive waveguide iris is employed additionally to improve the bandwidth (Type B). The results of both steps are shown in this section. 
(a)

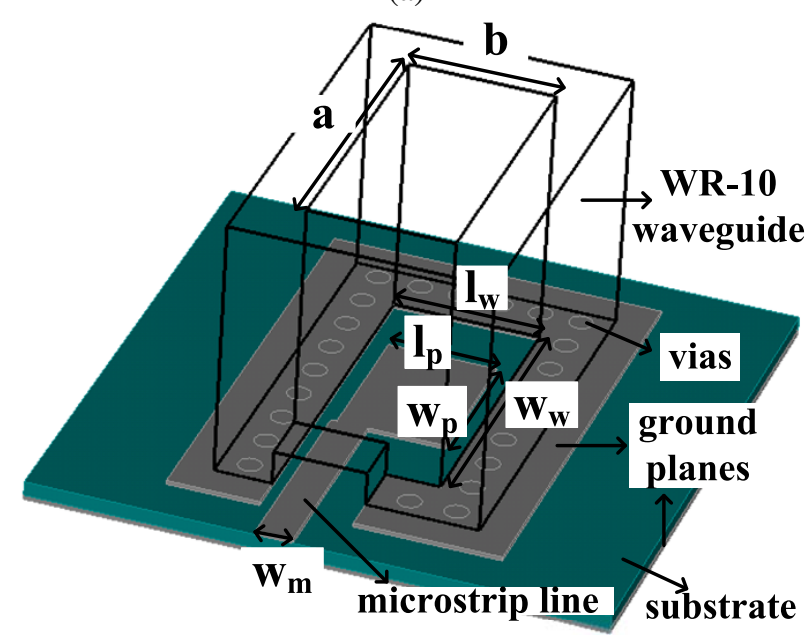

(b)

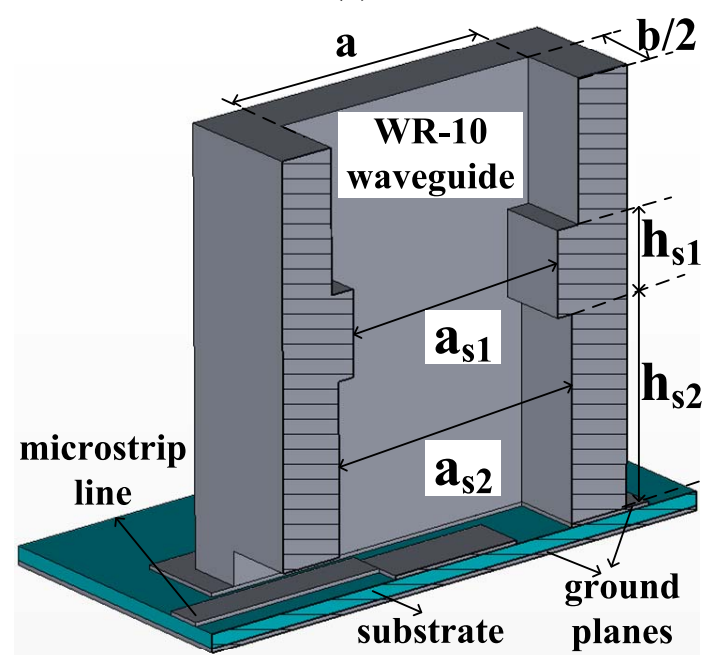

Figure 4.15.: 3D structures of the proposed vertical transitions (The height of each waveguide flange is $6 \mathrm{~mm}$.): (a) The simple transition without the waveguide iris (Type A), (b) The transition with the waveguide iris (Type B).

\subsubsection{Motivation}

Several waveguide to microstrip line transitions have been reported in the literature. The transition based on the backshorted waveguide [62] is very broadband, but an additional short circuited waveguide is needed on the back-side of the PCB. In [63], a probe coupling is used for a very broad bandwidth transition. However, such transitions suffer from manufacturing or fabrication complexity. The transitions, used in [64] and [65], are based on the slot-coupling technique. They provide broadband performances, but require multi-layer structures with additional radiating patches within the waveguide.

In [66] and [67], gap coupled parasitic patch antennas are used for the transition of a microstrip line to a WR-10 waveguide. However, they consist of a multi-section impedance transformer possessing numerous critical dimensions, which make the transition very sensitive to fabrication errors.

In this work, two mm-wave microstrip line to perpendicular air-filled WR-10 waveguide transitions are designed, as shown in Fig. 4.15. The first configuration, shown in Fig. 4.15(a), is based upon the ease of machining and the second one, shown in Fig. 4.15(b), is designed to cover a broader frequency bandwidth. The cross-sectional configurations of these transitions including waveguide flanges are shown in Fig. 4.16. Key features of the proposed designs are the use of a grounded single layer substrate using the standard PCB fabrication process, a new impedance matching topology, and compactness thanks to a feeding technique through the H-plane wall of the waveguide. 
(a) Cross-section of a transition without stepped transformer.

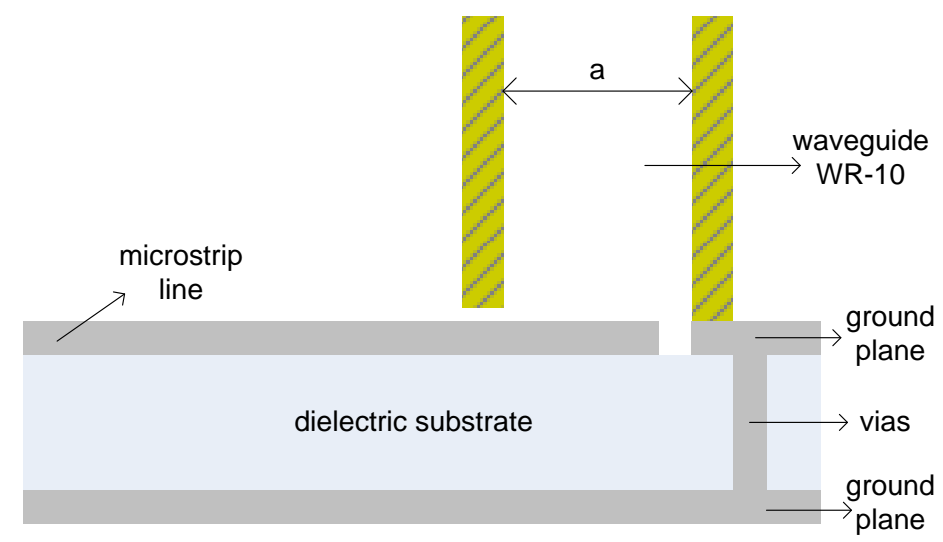

(b) Cross-section of a transition with stepped transformer.

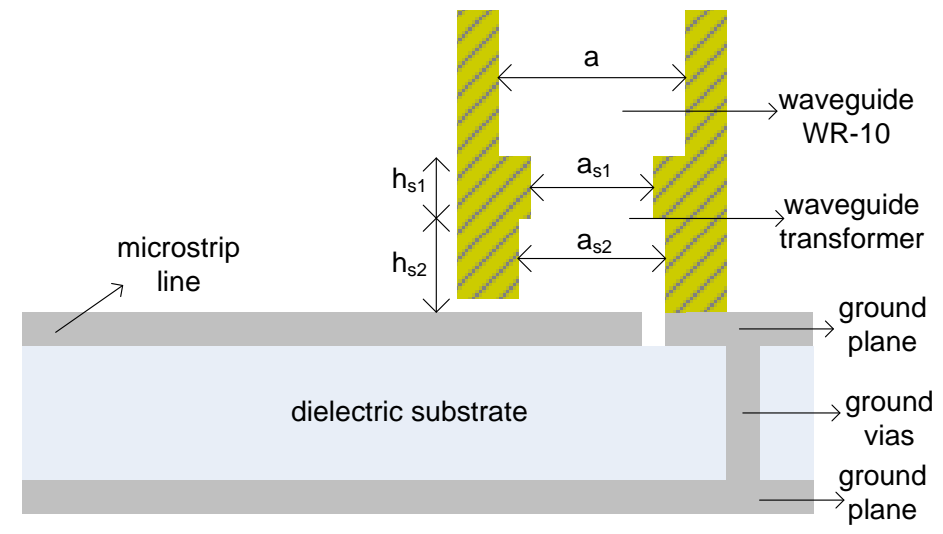

Figure 4.16.: Cross-sections of two different waveguide transitions.

\subsubsection{Design Procedure}

The goal of the first design is to develop the most cost effective transition. Hence mm-wave signals, carried via a $50 \Omega$ microstrip line, are transformed to a perpendicular air-filled WR10 rectangular waveguide on the same side. In order to convert the electric fields of the quasi-TEM fundamental mode of the microstrip line to electric fields of the $\mathrm{TE}_{10}$ fundamental mode of the rectangular waveguide, a patch antenna having electric fields of the $\mathrm{TM}_{01}$ fundamental mode is used as a matching element. Fig. 4.15(a) and Fig. 4.17(a) show the first type of the transitions including the waveguide flange and the top view of the planar circuit, respectively. The transitions are designed using a $0.127 \mathrm{~mm}$ thick RO3003 substrate and a full-wave electromagnetic simulation tool, CST Microwave Studio. The substrate has been characterized using microstrip ring resonators and microstrip lines with various lengths. The parameters are extracted from the measurement results. The dielectric permittivity $\left(\epsilon_{r}\right)$ and the loss tangent $(\tan \delta)$ are taken as 3.15 and 0.01 within the frequency of interest, respectively.

Detailed dimensions of the transition without the waveguide iris (Type A) are (in mm): 
(a)

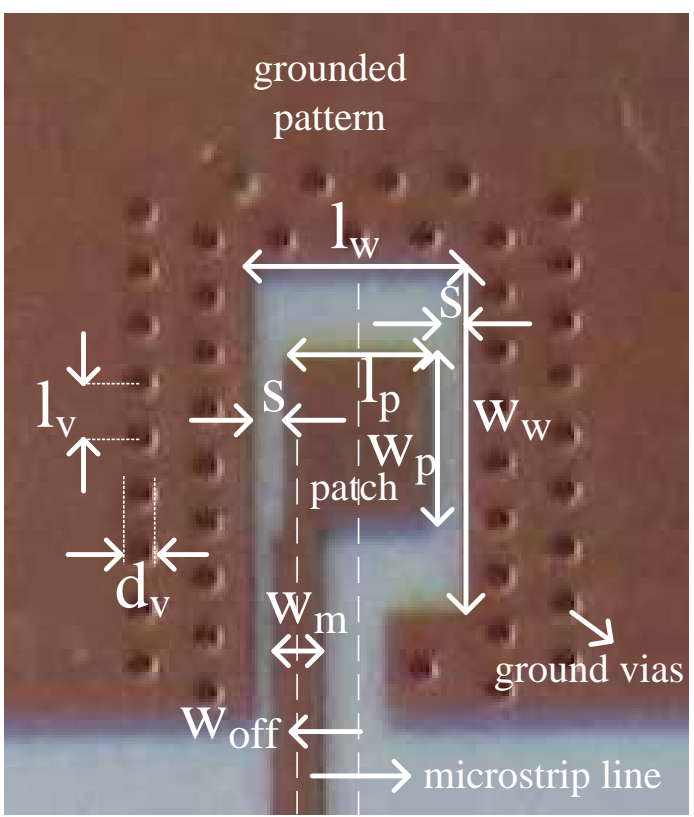

(b)

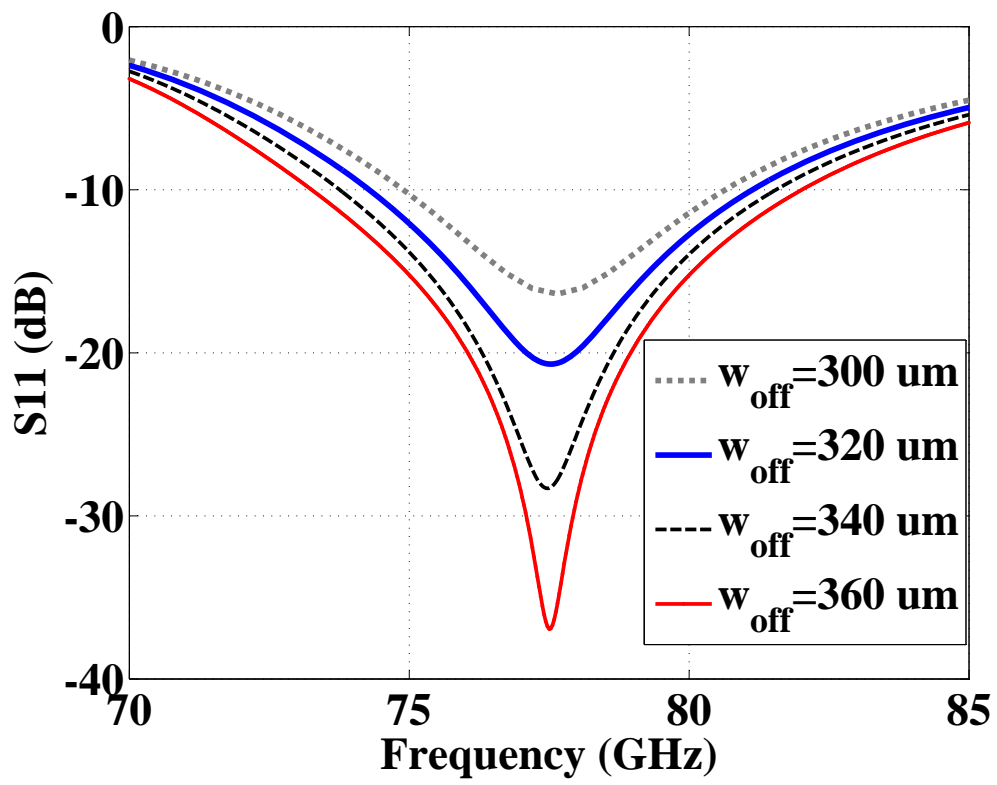

Figure 4.17.: (a) Top view of the fabricated planar circuit used for the both waveguide transitions, (b) Effect of the parameter $\mathrm{w}_{\text {off }}$ on impedance matching of the transition without iris (Type A).

$l_{p}=0.94, w_{p}=1.3, w_{m}=0.32, l_{g}=0.11, w_{\text {off }}=0.36, a=2.54, b=1.27$. None of the values listed above exceeds standard PCB fabrication design rules (line width and spacing $>100 \mathrm{um}$ ). The dimensions of the microstrip line $\left(w_{m}\right)$ and the WR-10 waveguide $\left(w_{w}, l_{w}\right)$ are fixed.

To avoid the use of an additional matching circuit, the $50 \Omega$ microstrip line is fed directly 


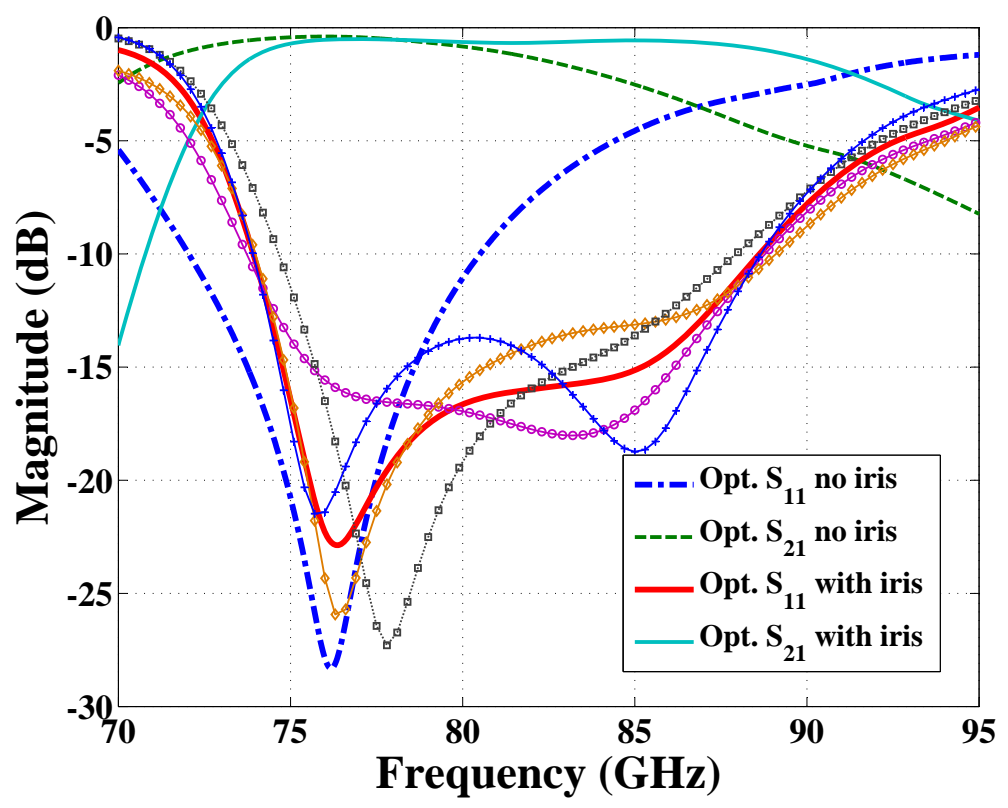

Figure 4.18.: Simulated S-parameters of the transitions (curve with circle: $\mathrm{S}_{11}$ for $\Delta \mathrm{a}_{s 1}=+60$ um , curve with diamond: $S_{11}$ for $\Delta a_{s 1}=-60 \mathrm{um}$, curve with square: $S_{11}$ for $\Delta \mathrm{h}_{s 1}=-200 \mathrm{um}$, curve with square: $\mathrm{S}_{11}$ for $\Delta \mathrm{h}_{s 1}=+200 \mathrm{um}$ ).

at the non-radiating edge of the patch with an offset value $\left(w_{o f f}\right)$, as shown in Fig. 4.17(a). Since the patch antenna is surrounded with a ground plane, it acts like a coplanar patch antenna. The slot between the radiating edge and ground plane $(s)$ and feed location at the non-radiating edge $\left(w_{o f f}\right)$ determine both impedance matching and wider bandwidth [68]. After optimization of the length of the patch $\left(l_{p}\right)$ and the slot $(s)$ for the resonant frequency at $77 \mathrm{GHz}, \mathrm{w}_{\text {off }}$ is used to maximize the bandwidth, as depicted in Fig. 4.17(b). Changing the width of the patch $\left(w_{p}\right)$ from $1.25 \mathrm{~mm}$ up to $1.4 \mathrm{~mm}$ only resulted in an impedance bandwidth variation of $1.2 \mathrm{GHz}$, determined from $10 \mathrm{~dB}$ return loss. The simulated return and insertion losses of the transition can be found in Fig. 4.18.

Fig. 4.17(a) shows a number of grounded vias placed around the patch with a spacing of 0.4 $\mathrm{mm}\left(\mathrm{l}_{v}\right)$ and a diameter of $0.25 \mathrm{~mm}\left(\mathrm{~d}_{v}\right)$ to suppress parallel plate modes in the dielectric substrate. The exact placement and diameter of the ground vias was found to be not critical for the overall performance of the transition. The insertion loss for the transition with ground vias spaced with $0.8 \mathrm{~mm}\left(l_{v}\right)$ is $0.1 \mathrm{~dB}$ more within the frequency band of interest. The size of the waveguide opening above the microstrip feed line is chosen large enough as not to disturb the propagation of millimeter-wave signals on the microstrip line.

In the work presented in [69], a capacitive waveguide tuning step is added into the waveguide for a broadband operation. However, the capacitive tuning step is sensitive to fabrication tolerances. In our work, a novel inductive iris is placed into the waveguide to tune out the reactance of the characteristic impedance, as shown in Fig. 4.15(b), since the bandwidth is less 
sensitive to fabrication errors. Variation of height $\left(\Delta h_{s 1}= \pm 200 \mathrm{um}\right)$ and width $\left(\Delta a_{s 1}= \pm 60\right.$ um) of the iris against return loss of the transition is shown in Fig. 4.18.

The dimensions of the transition employing the waveguide iris (Type B) are (in mm): $l_{p}=0.9$, $w_{p}=1.25, w_{m}=0.32, l_{g}=0.12, w_{o f f}=0.37, a=2.54, b=1.27, a_{s 1}=2.1, h_{s 1}=0.75, a_{s 2}=2.4$, and $h_{s 2}=1.8$. The dimensions of the patch $\left(w_{p}\right.$ and $\left.l_{p}\right)$ are varied slightly to improve the impedance matching. Fig. 4.18 shows the simulated insertion and return losses of the optimized transition.

\subsubsection{Assembly and Measurements}

The two types of waveguide transition were fabricated and tested. To measure the performance, a back-to-back configuration was employed. This configuration contains two microstrip-to-waveguide transitions connected to each other using a $50 \mathrm{~mm}$ long $50 \Omega \mathrm{mi}$ crostrip line to avoid interactions between the transitions, as shown in Fig. 4.19(a). Fig. 4.19(b) shows the fabricated waveguide flanges connected to the RF board and the manufactured microstrip lines with coplanar coplanar GSG probe pad structures. These metallic waveguide flanges were fabricated using regular CNC milling. To de-embed the line loss of the $50 \mathrm{~mm}$ long $50 \Omega$ line from the back-to-back configuration, another test circuit was built consisting of the $50 \mathrm{~mm}$ long line with coplanar GSG probe pads.

The test circuit consisting of the line has been measured with a vector network analyzer using W-band waveguide on-wafer probes for the frequency range from $70 \mathrm{GHz}$ up to $90 \mathrm{GHz}$. The S-parameter measurements have been corrected using a TRL calibration on the substrate.

The insertion loss of the $50 \mathrm{~mm}$ long microstrip line, which is measured as $1.2 \mathrm{~dB} / \mathrm{cm}$ at 77 $\mathrm{GHz}$, is de-embedded from the measurement results.

After performing waveguide LRL (line-reflect-line) calibration at the rectangular waveguide ends of the back-to-back transition, measured and simulated results of the transition without the iris are shown in Fig. 4.20. This transition has a bandwidth of $8.5 \mathrm{GHz}(11 \%)$ for a $10 \mathrm{~dB}$ return loss with an insertion loss better than $1.1 \mathrm{~dB}$ and a minimal insertion loss of $0.35 \mathrm{~dB}$ at $77 \mathrm{GHz}$. Fig. 4.21 shows the measured and simulated results of the transition with the iris. The return loss values are better than $10 \mathrm{~dB}$ in a $12 \mathrm{GHz}(15 \%)$ bandwidth with an insertion loss better than $1 \mathrm{~dB}$. The minimal insertion loss of one transition is $0.6 \mathrm{~dB}$ at $77 \mathrm{GHz}$. 
(a)

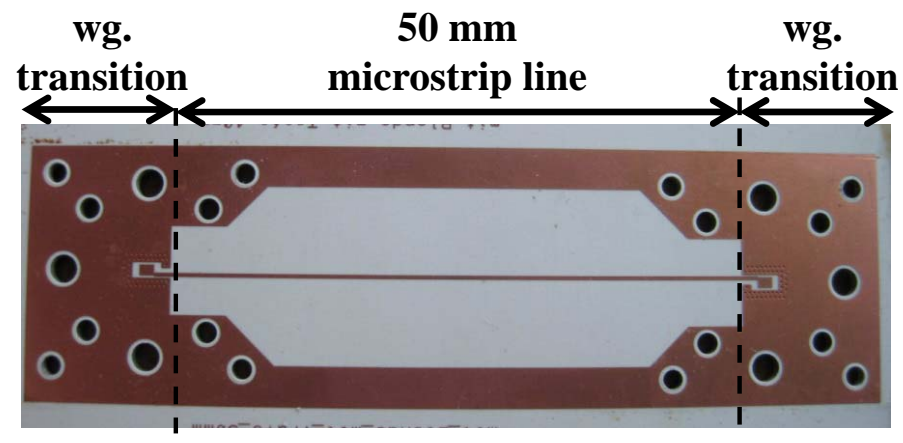

(b)

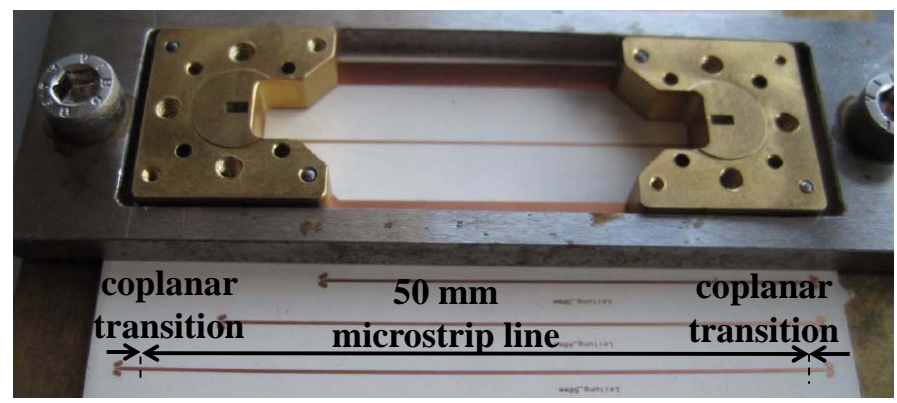

Figure 4.19.: Manufactured RF board including the waveguide flanges: (a) Fabricated RF board for back-to-back assembly, (b) Metallic waveguide flanges connected to the RF board and microstrip lines with coplanar transitions on the same board.

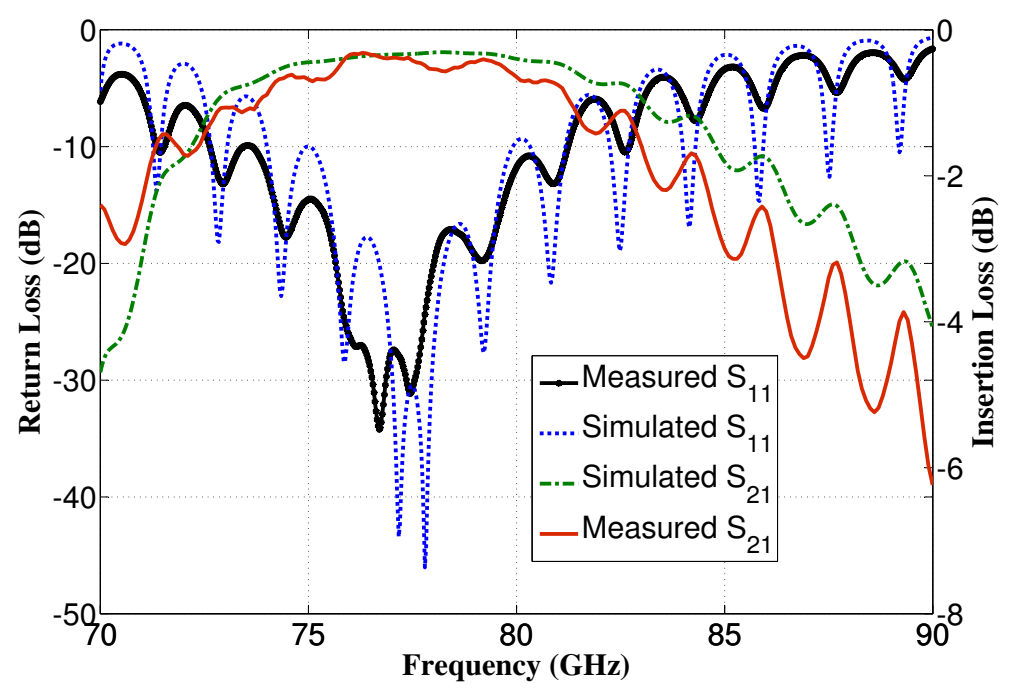

Figure 4.20.: Simulated and measured results of the transition without iris (Type A). 


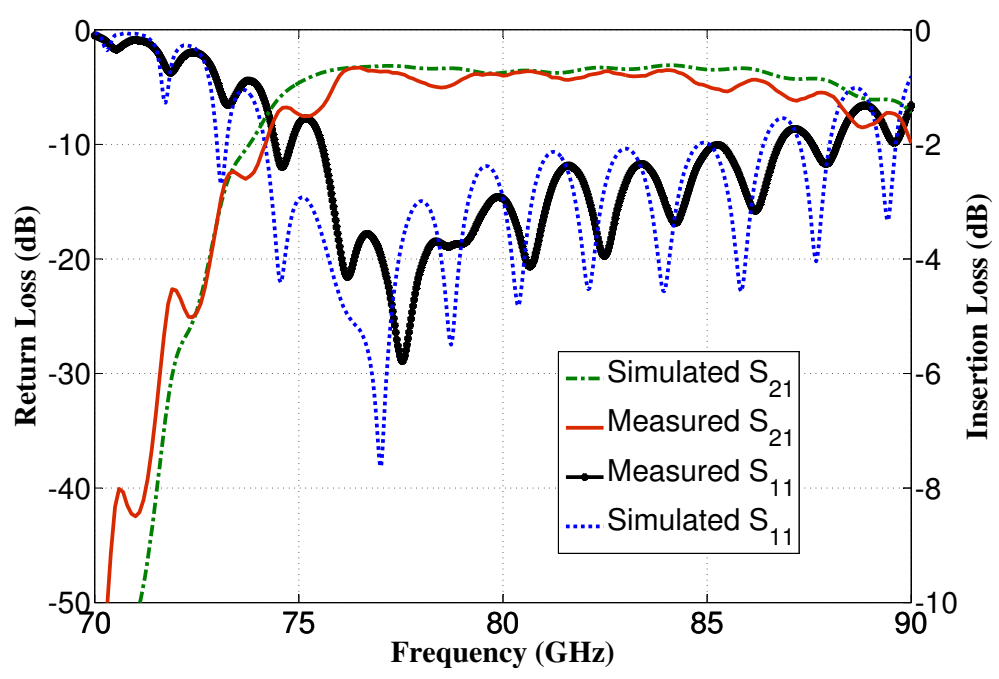

Figure 4.21.: Simulated and measured results of the transition with iris (Type B).

After characterization of the transitions, the transition employing the iris within the waveguide (Type B) is employed for the measurement test setups that will be explained in the next chapter in detail.

\subsection{Millimeter-wave planar balun}

The dual-fed phased array demonstrator consists of 77-GHz transceiver MMICs in a fully differential circuit configuration. Since the single-ended linear array antenna and waveguide transitions are employed in this work, a balanced-to-unbalanced transformer (Balun) is required. Hence, a balun is characterized at the $77 \mathrm{GHz}$ frequency band in this section. Fig. 4.22(a) shows the layout of this planar millimeter-wave balun based on rat-race coupler principle. The $180^{\circ}$ phase difference ports are connected to the differential lines and the sum port acts as the output.

As seen from Fig. 4.22(a), the differential input ports are adjusted to match to wire bonds. The other port is a single ended output. Simulation was carried out using back-to-back connected baluns, connected to each other through their differential ports. The basic parameter of the balun design is only the diameter of the rat-race (the line length of the ring structure). The single-ended port has $50 \Omega$ line impedance, and the differential ports are fed by a taper to yield a parallel differential line with an odd-mode impedance of $100 \Omega$ substrate.

Fig. 4.23 shows the full-wave simulation and measurement results of the back-to-back configuration between 70 and $85 \mathrm{GHz}$. Measured losses are in good agreement with the simulated ones. According to these results, the insertion loss can be approximately calculated as the half value of the insertion loss, contributed by one balun. The measured insertion loss is 0.8 $\mathrm{dB}$ at $77 \mathrm{GHz}$. The measured return losses are better than $-15 \mathrm{~dB}$ in the frequency band of 70-85 GHz. 
(a) Layout.

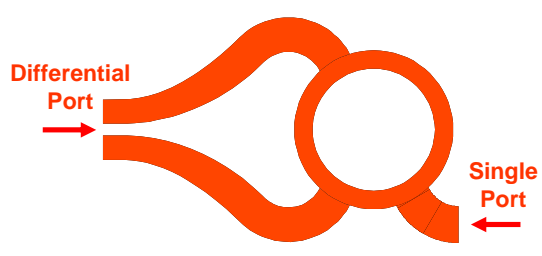

(b) Realized baluns.

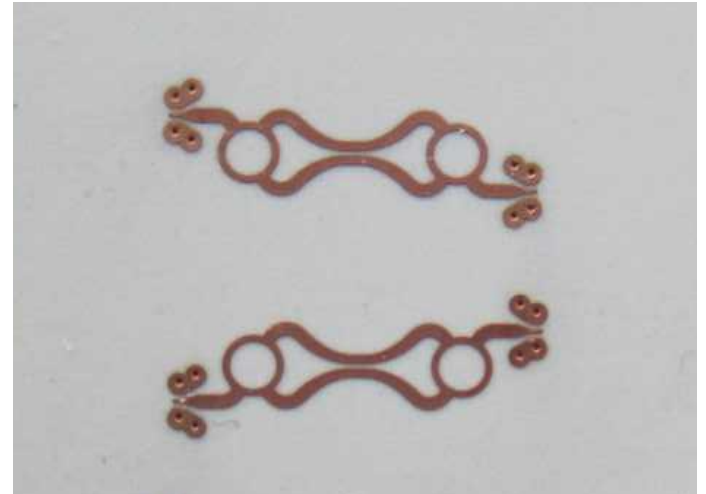

Figure 4.22.: Back to back test setup for baluns connected to each other for characterization.

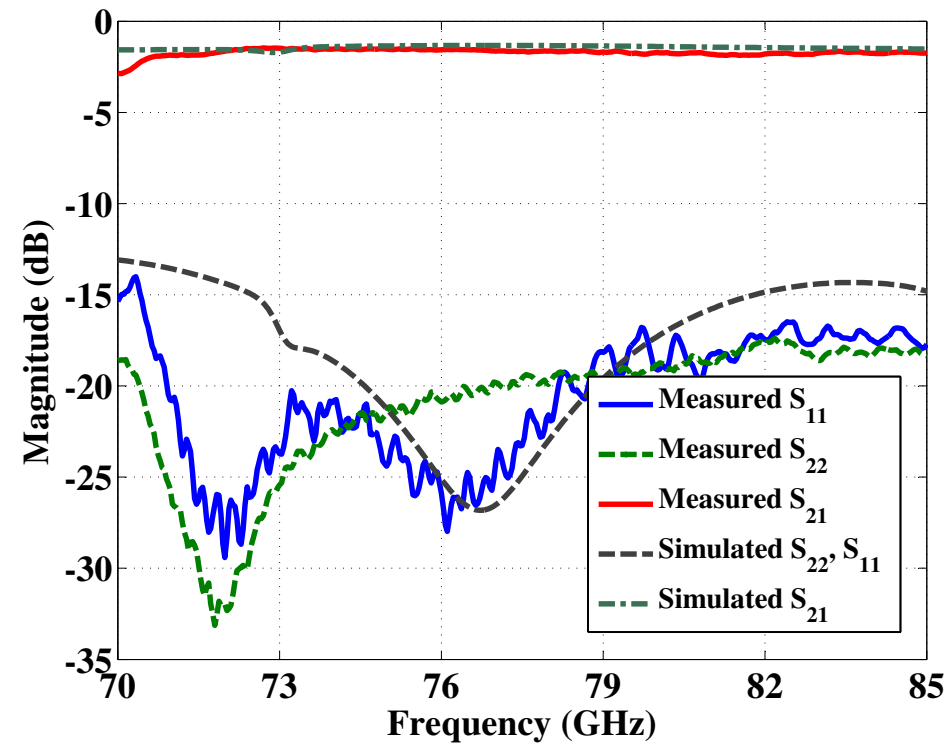

Figure 4.23.: Measured and simulated insertion and return losses of connected baluns. 


\section{Test and Measurements}

In this chapter, the experimental results of a dual-fed phased array concept are presented. Two different measurement setups were designed and built to synthesize the desired antenna characteristics. Phase shifting and amplitude change are carried out using commercial passive WR-10 waveguide phase shifter and variable attenuator in the first setup. In this setup, a linear array antenna employing $N=6$ patch elements (with amplitude tapering and $L_{f}=1.41 \mathrm{~mm}$ ) is measured. In the second setup, an I-Q vector modulator is used for the change of phase and amplitude values in the transmitter path. In this setup, a linear array antenna employing $N=5$ patch elements (without amplitude tapering and $L_{f}=1.5 \mathrm{~mm}$ ) is measured.

\subsection{Setup based on passive WR-10 attenuator and phase shifter}

\subsubsection{Test configuration}

Figure 5.1 shows the first antenna measurement setup employing passive commercial waveguide phase shifter and variable attenuator. It is built in the anechoic chamber. Since apertures of these two antennas are extremely small and these antennas should be placed accurately for a correct measurement, a special measurement platform was built. Since the E- and H-planes of the desired antenna are to be measured, the RF-board housing the dual-fed antenna is fixed on a measurement platform. This measurement platform is situated on a turning table, which is turned by a step motor. This step motor is controlled by a microcontroller enabling a remote control of the turning table.

The dual-fed antenna is measured as a transmit antenna. Mm-wave signals are generated by a W-band signal generator $(75-110 \mathrm{GHz})$. The frequency and amplitude of mm-wave signals are remotely controlled over GPIB on a PC. However, micrometer drives of the WR-10 phase shifter and variable attenuators are adjusted manually for each antenna pattern based on the look-up table which has already been mentioned in Section 4.2. Fig. 5.2(a) shows a photograph of a transmit antenna placed on the measurement platform. This platform can be turned around itself with $\pm 180^{\circ}$ by a step motor. Since the thickness of the RF-board is so thin, it is fixed on the measurement platform made up of Polyoxymethylene (POM) via plastic screws. All test and measurement devices and waveguide components are placed behind the measurement platform through waveguide bends, as shown in Fig. 5.2(b).

An E-band (60-90 GHz) horn antenna is used as a receiver antenna. The received signals are down-converted via an external harmonic mixer from Rohde \& Schwarz and delivered to 
a spectrum analyzer to measure radiation pattern. The frequency and amplitude of received signals are saved over GPIB on a PC. Since the minimum far field distance is defined as a function of the maximum dimension of the antenna and the frequency [70];

$$
D \geq \frac{2 L^{2}}{\lambda}
$$

The desired antenna is designed at $77 \mathrm{GHz}$ with a length of $15 \mathrm{~mm}(L)$. Therefore, the distance between the receiver and transmitter antennas $(D)$ is taken as $1 \mathrm{~m}$, which satisfies the far field conditions.

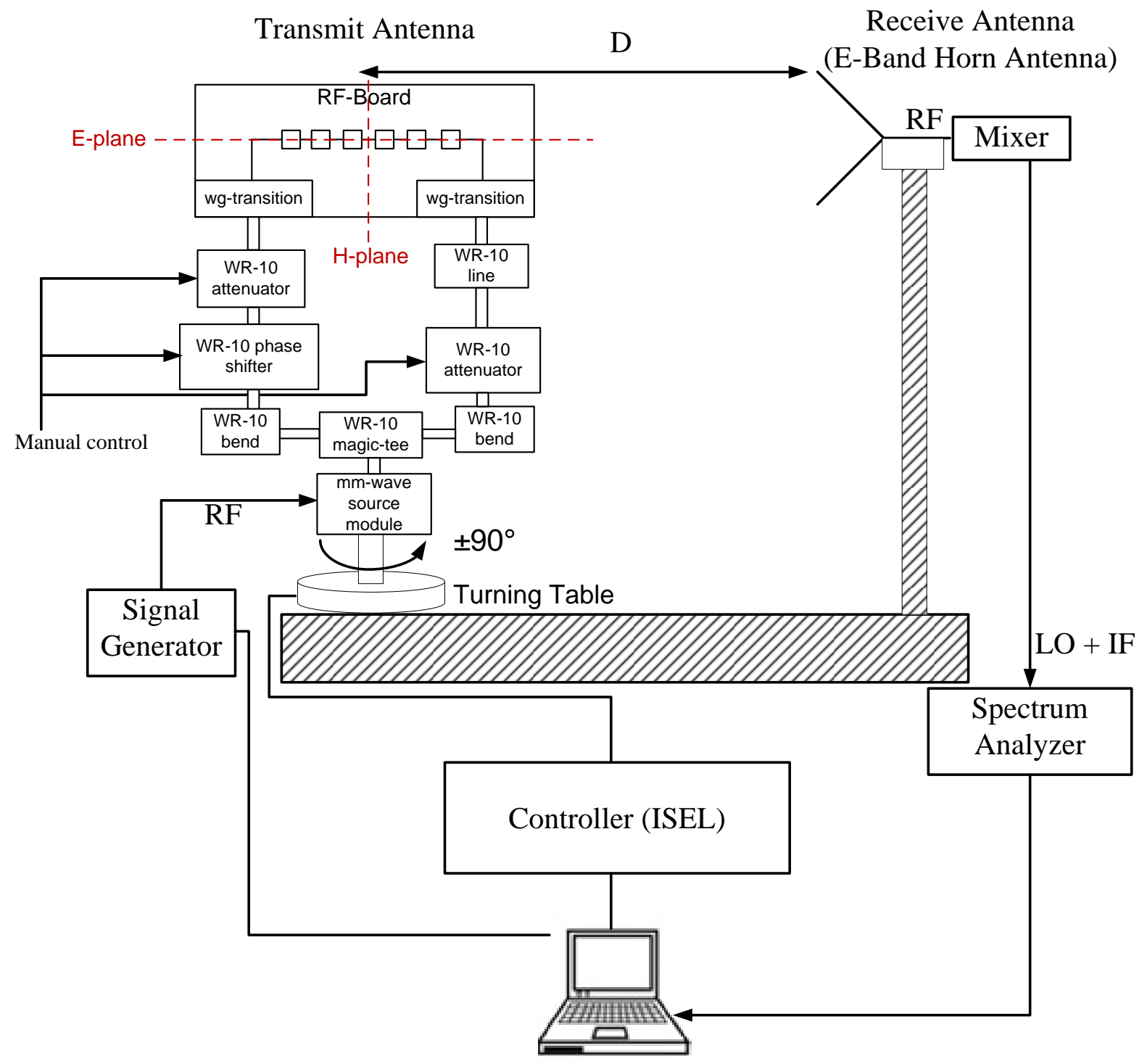

Figure 5.1.: Antenna measurement setup employing waveguide phase shifter. 
(a) Front part employing planar RF board.

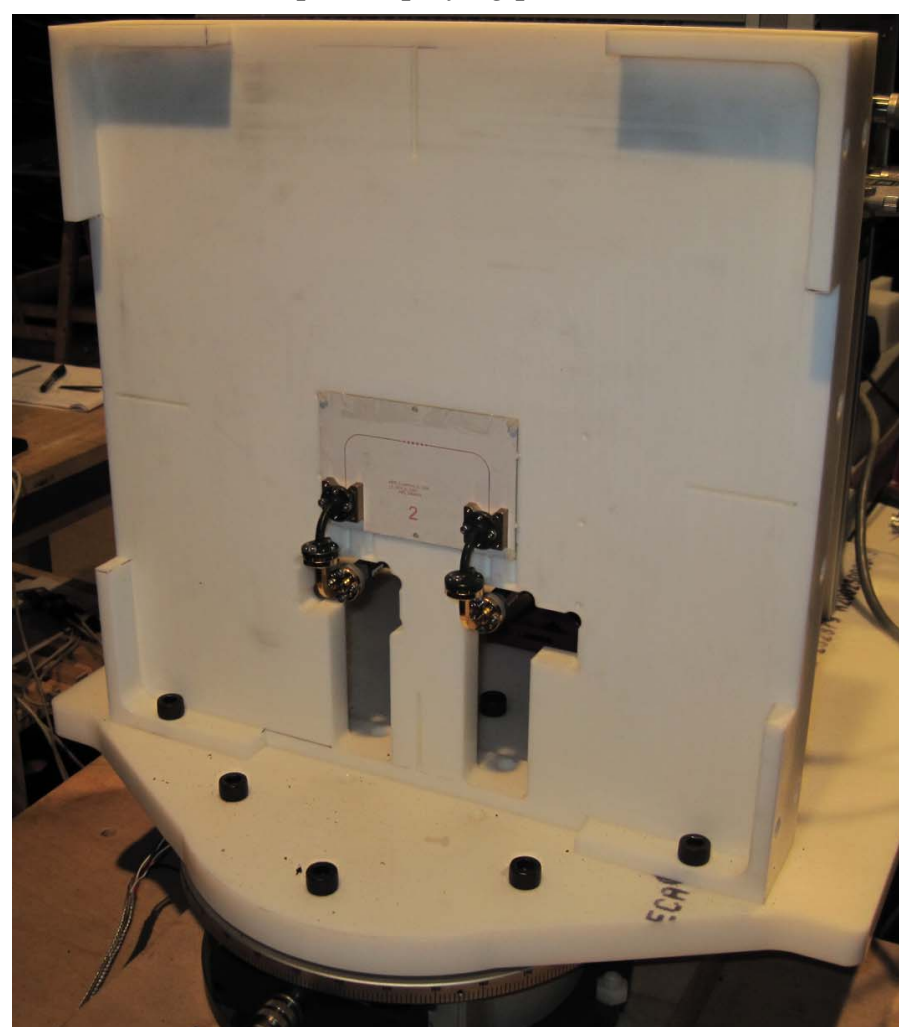

(b) Back part employing waveguide components.

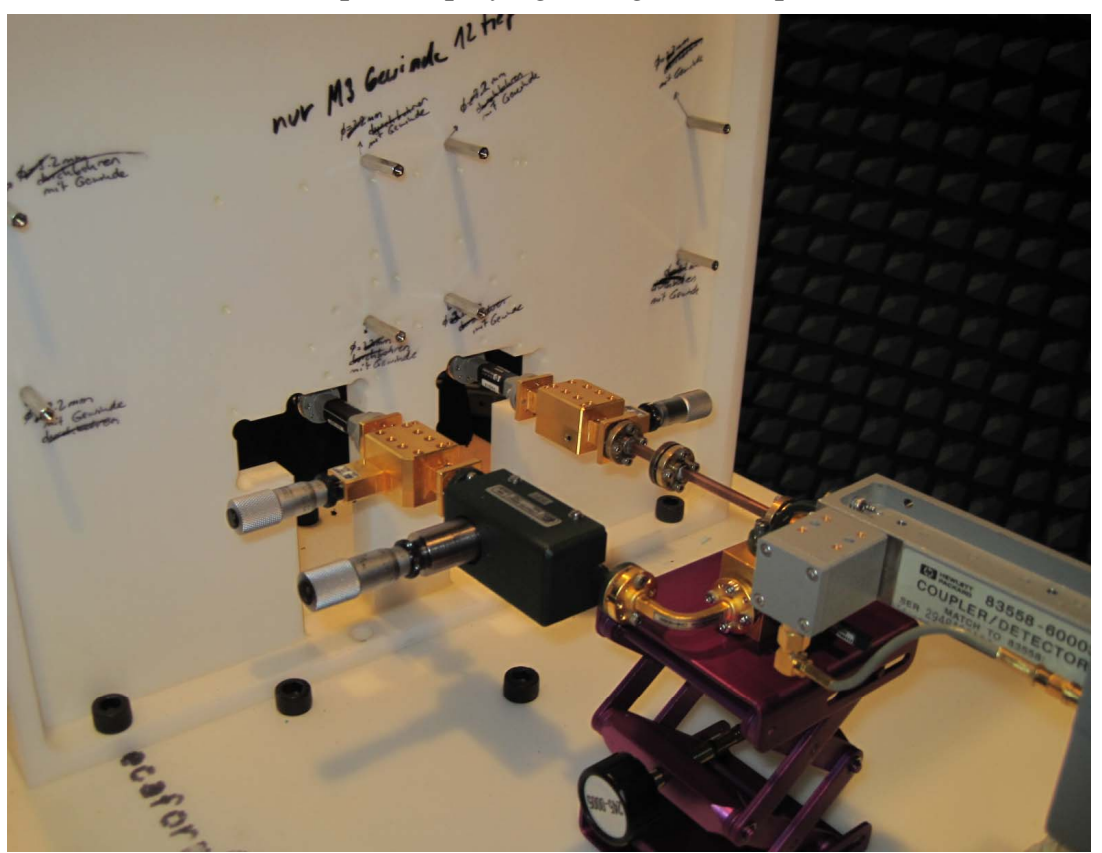

Figure 5.2.: Measurement platform for setup based on waveguide phase shifter and attenuator. 


\subsubsection{Antenna with amplitude tapering}

A linear array antenna, made up of 6 patch elements, is designed and characterized. Table A.1, Table 5.1 and Fig. A.2 show parameters of the designed array. Since the amplitude tapering is applied to the array, the widths of the patches are different. Fig. 5.3(a) shows the RF board housing the designed array and the designed waveguide transitions, interconnected with each other using long $50 \mathrm{ohm}$ microstrip lines. The purpose of placing waveguide transitions in the H-plane of the antenna is to avoid disturbing the E-plane radiation since a beam steering is performed in the E-plane. The measured S-parameters are shown in Fig. 5.3(b) using a 2-port network analyzer with waveguide ports between $70 \mathrm{GHz}$ and $90 \mathrm{GHz}$.

Table 5.1.: Parameters of a dual fed array design with amplitude tapering at $77 \mathrm{GHz}$

\begin{tabular}{c|c}
\hline Design parameter & Value \\
\hline \hline Number of patches $(N)$ & 6 \\
\hline$L_{f}(\mathrm{~mm})$ & 1.41 \\
\hline$L_{p}(\mathrm{~mm})$ & 1.09 \\
\hline$w_{f}(\mathrm{~mm})$ & 0.1 \\
\hline$w_{p}(\mathrm{~mm})$ & $0.82,1.07,1,2$ \\
\hline
\end{tabular}

\subsubsection{Measurement results}

Figure 5.4 shows the normalized measured and simulated sum / difference patterns in the E-plane. Measurements are carried out at the operating frequency of $77 \mathrm{GHz}$ within the field of view of $\pm 40^{\circ}$ with $\pm 1^{\circ}$ steps, whereas simulations are performed between $\pm 90^{\circ}$. The amplitude value $\left(A_{2}=A_{1}\right)$ of each feeding is taken to be the same for each pattern. If a phase difference $\left(\Delta \phi=\phi_{2}-\phi_{1}\right)$ between these two feedings is adjusted to $180^{\circ}$, a sum pattern in the broadside is generated. If phase difference is $0^{\circ}$, a dual-beam (difference) pattern is generated which looks at the $\pm 10^{\circ}$ directions. The sum pattern is $3 \mathrm{~dB}$ higher than the difference pattern since the power of difference pattern is divided into two equal values to generate two beams. The gain difference between the sum and difference patterns is almost $34 \mathrm{~dB}$ at the broadside. This design can be used to steer null beam to the broadside direction with a dynamic range better than $34 \mathrm{~dB}$. Simulation and measurement results for both patterns agree well with each other.

The maximum measured beam steering angle with this antenna configuration is $\pm 8^{\circ}$ in the Eplane, when amplitude ratio and phase difference are adjusted to the given values, as shown in Fig. 5.5. Even though a continuous beam steering with very high resolution can be achieved by using the analog phase shifter and variable attenuator, in this figure only 3 patterns with different directions are plotted. The measured and simulated patterns are plotted in Fig. 5.5(a) for the $0^{\circ}$ direction. The HPBW of the main beam is $17^{\circ}$ and the sidelobe levels are higher 
(a) RF board employing linear array antenna with waveguide transitions.

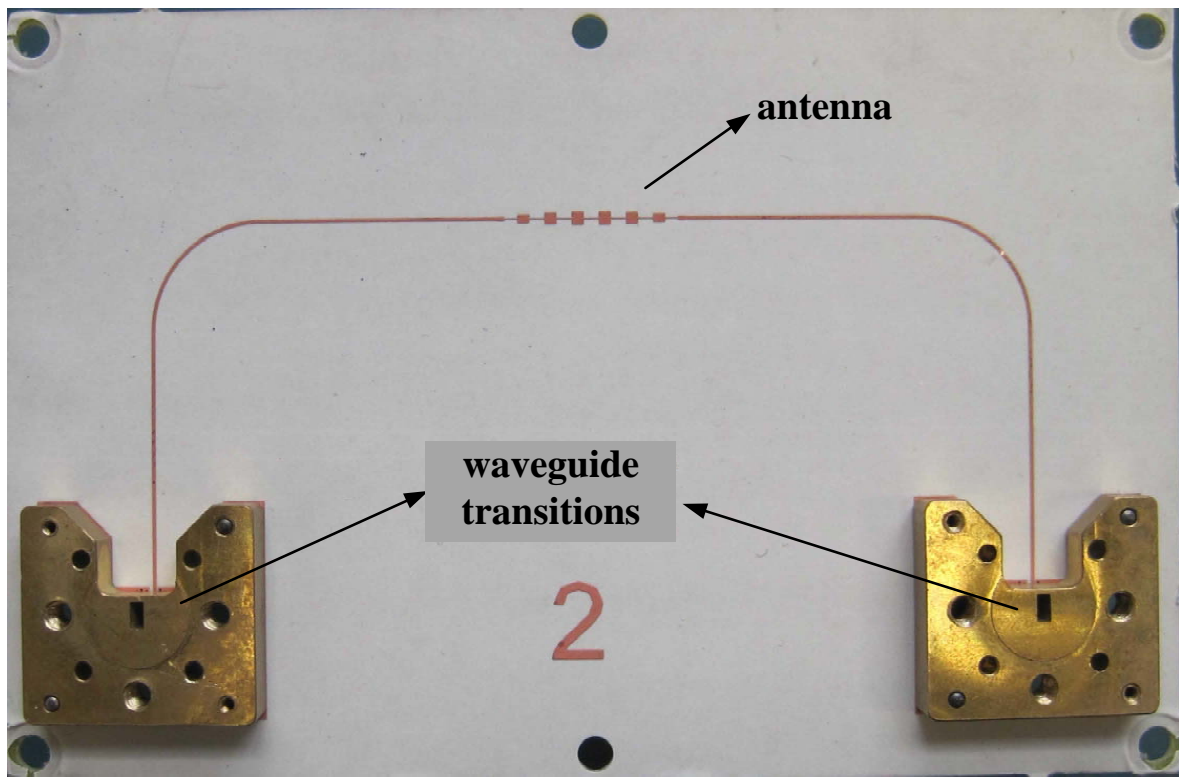

(b) Measured S-parameters of planar linear array antenna at the waveguide input ports.

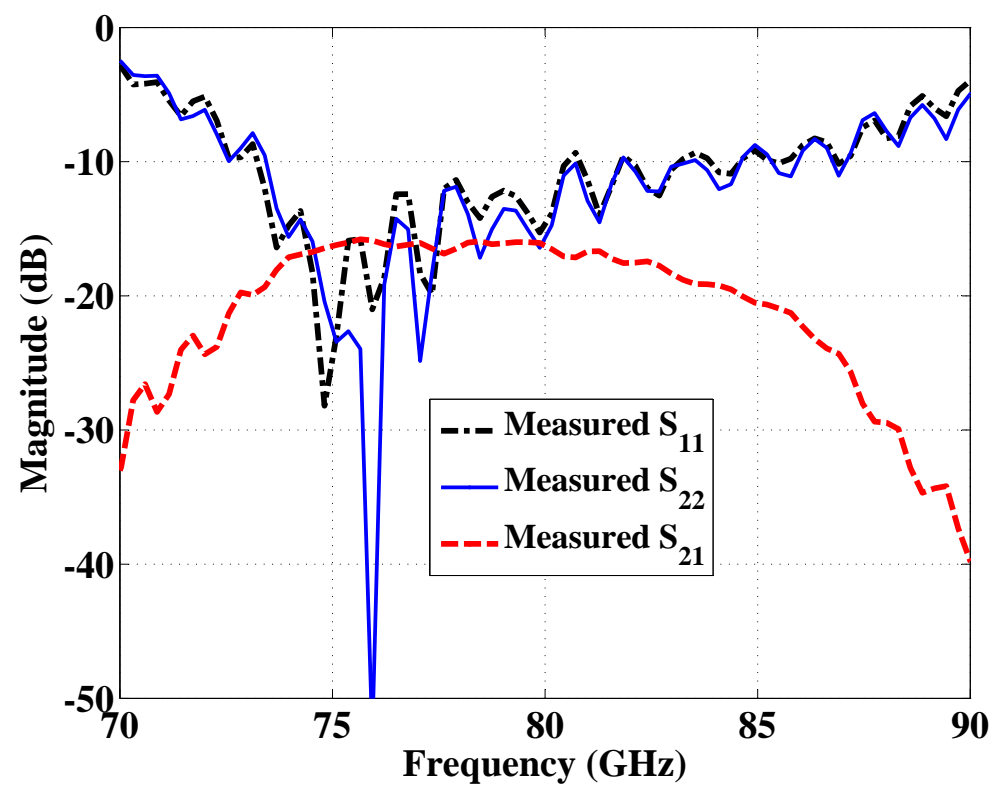

Figure 5.3.: Characterization of a linear array antenna including waveguide transitions.

than $22 \mathrm{~dB}$. Normalized patterns are plotted in Fig. 5.5(b) for the direction of $-4^{\circ}$. In this direction, the HPBW of the main beam is $17^{\circ}$ and the sidelobe levels are higher than $19 \mathrm{~dB}$. In Fig. 5.5(c), patterns are shown for the $-8^{\circ}$ direction. This pattern has the HPBW of $13^{\circ}$ and sidelobe levels of $15 \mathrm{~dB}$. The same pattern can also be obtained for $0^{\circ}$ to $8^{\circ}$ if the same amplitude and phase values are applied in the opposite channel. 


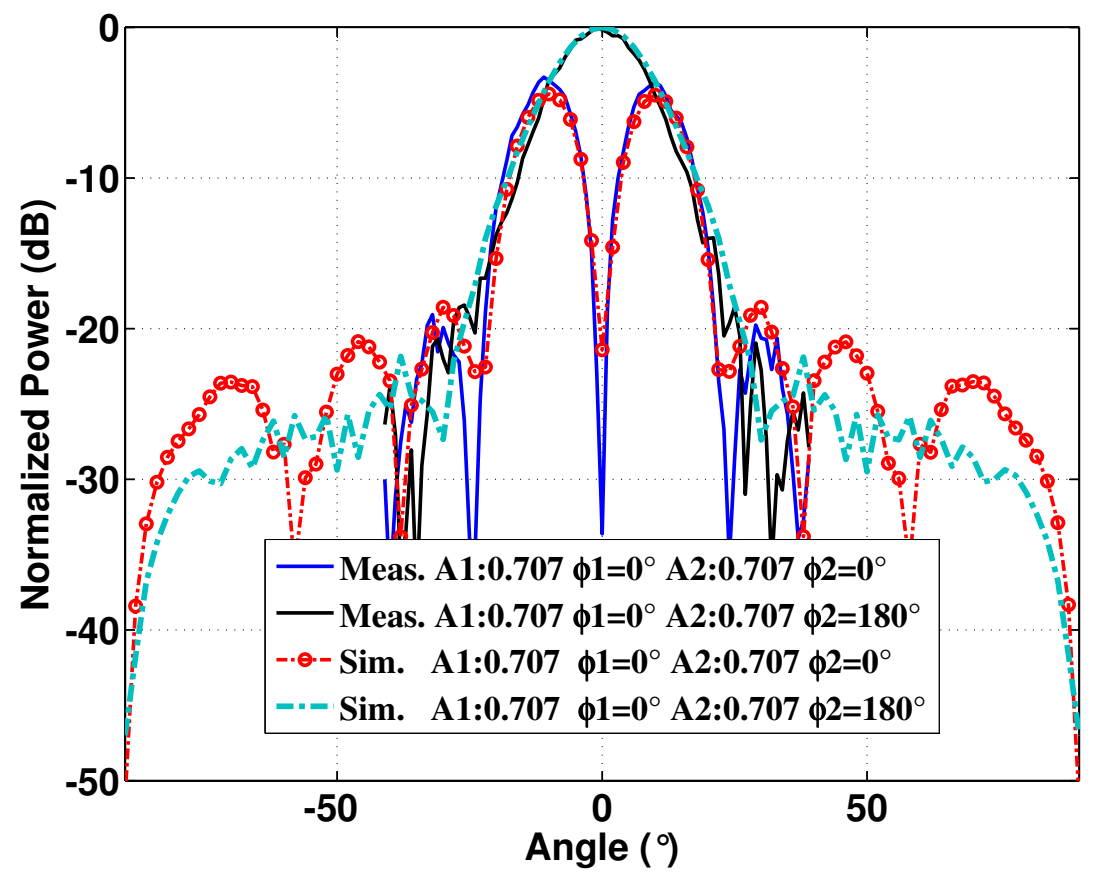

Figure 5.4.: Sum and difference antenna patterns $\left(A 2=A 1, \Delta \phi=\phi_{2}-\phi_{1}\right)$.

Fig. 5.6 shows measured and simulated $\mathrm{H}$-plane patterns for $A 1=A 2$ and $\Delta \phi=180^{\circ}$. As seen from this figure, measurement and simulation results agree well with each other in the field view of $0^{\circ}$ to $90^{\circ}$. However, measured pattern between $-90^{\circ}$ and $0^{\circ}$ is much lower than the expected pattern. The reason is the WR-10 waveguide bends used for bending signals with 180 degrees between waveguide transitions and other waveguide components in the setup. Due to signal reflections caused by these metallic waveguide components, the result is degraded at one half of the field view, as expected. Measured cross-polarized pattern of this signal is better than $30 \mathrm{~dB}$.

The maximum beam steering angle is $\pm 18^{\circ}$ in the E-plane, when the mm-wave signal is supplied only by one of the input ports and the operating frequency is changed from 76 to 81 $\mathrm{GHz}$ as shown in Fig. 5.7. The grating lobes are beginning to appear at $\pm 80^{\circ}$ if the frequency is increased. The normalized beam is steered at $\pm 10^{\circ}$ if one of two channels are turned off and the operating frequency is $78 \mathrm{GHz}$ as shown in Fig. 5.8(a). The sidelobe levels are higher than $13 \mathrm{~dB}$. Fig. 5.8(b) shows two beams directed at $\pm 18^{\circ}$. In this figure, it can be clearly seen that grating lobes appear within the field of view. Since an increase in frequency also causes an increase in electrical length between subsequent patches in the setup, these unwanted lobes also appear within the field of view. 
(a) Beam steering directed at $0^{\circ}$.

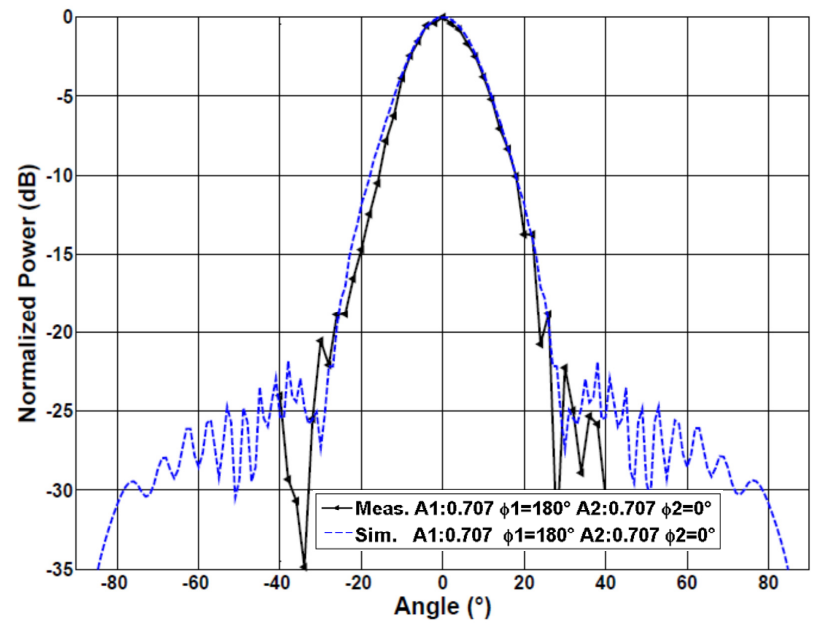

(b) Beam steering directed at $-4^{\circ}$.

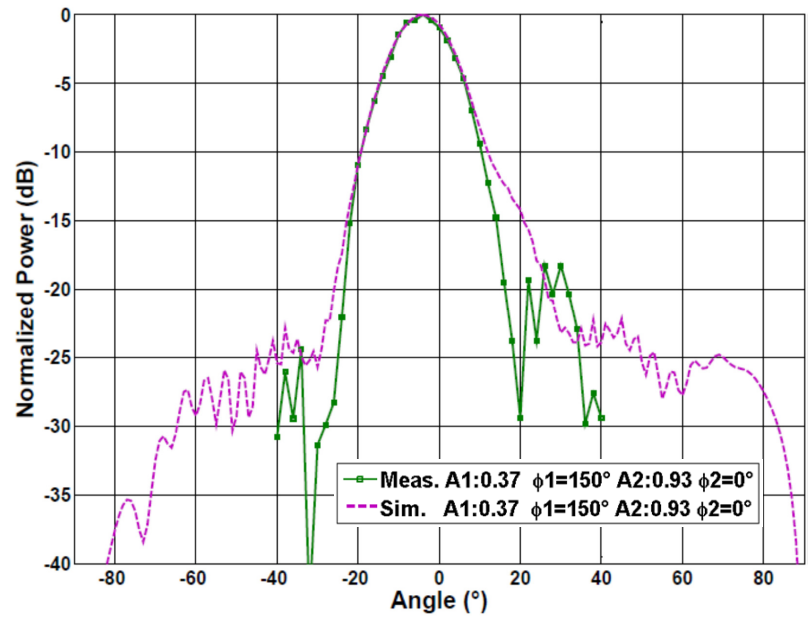

(c) Beam steering directed at $-8^{\circ}$.

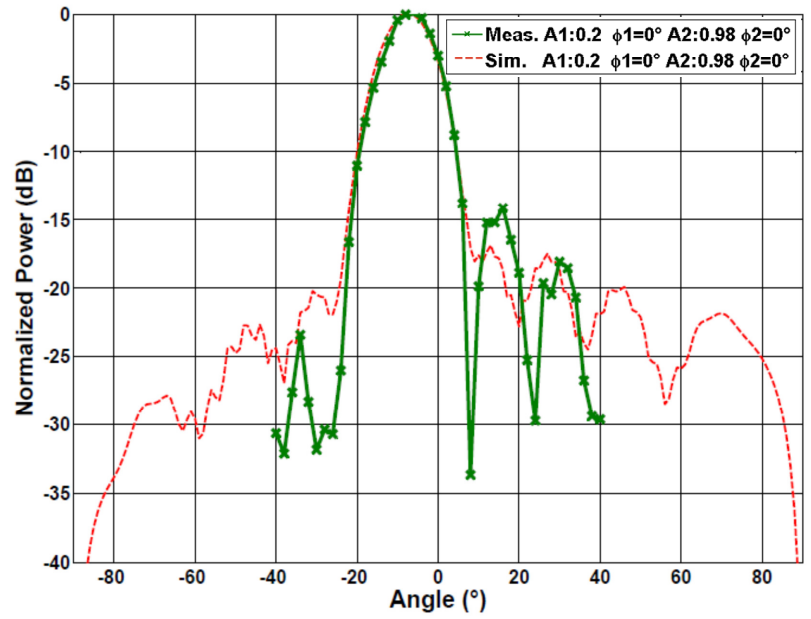

Figure 5.5.: Beam steering using a single phase shifter and attenuator. 


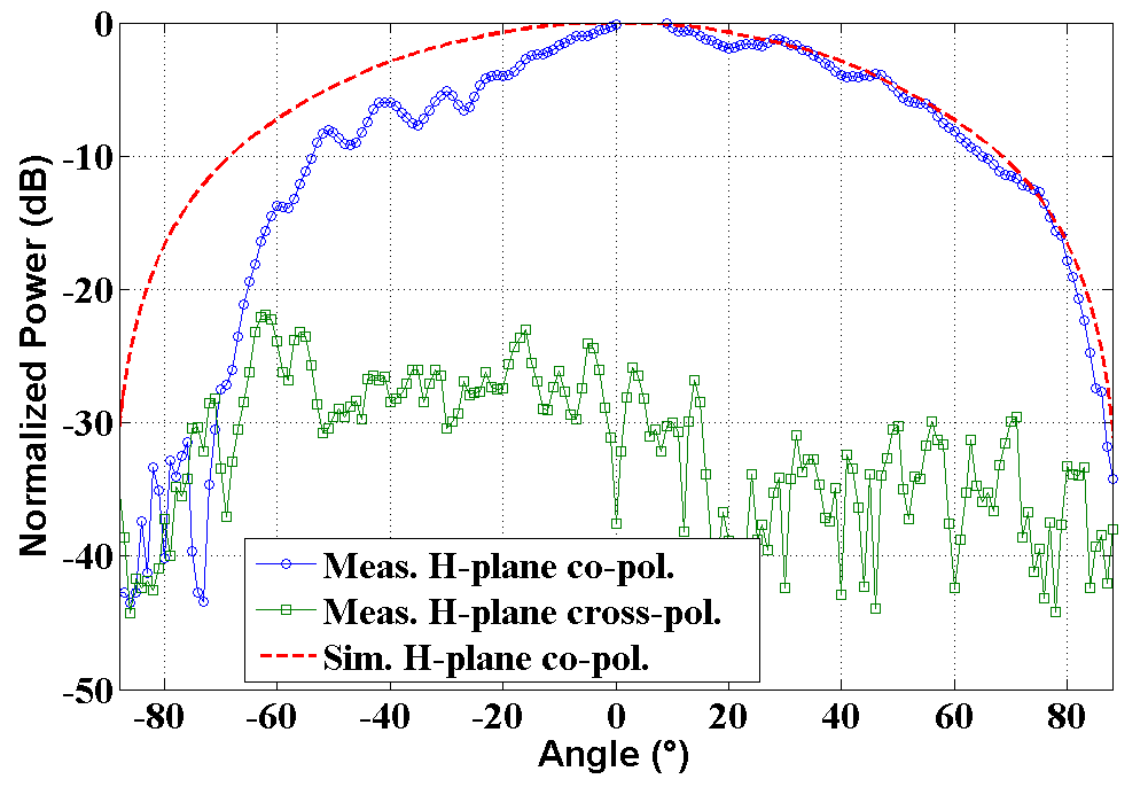

Figure 5.6.: H-plane radiation pattern $\left(A 1=A 2=0.707, \Delta \phi=180^{\circ}\right)$.

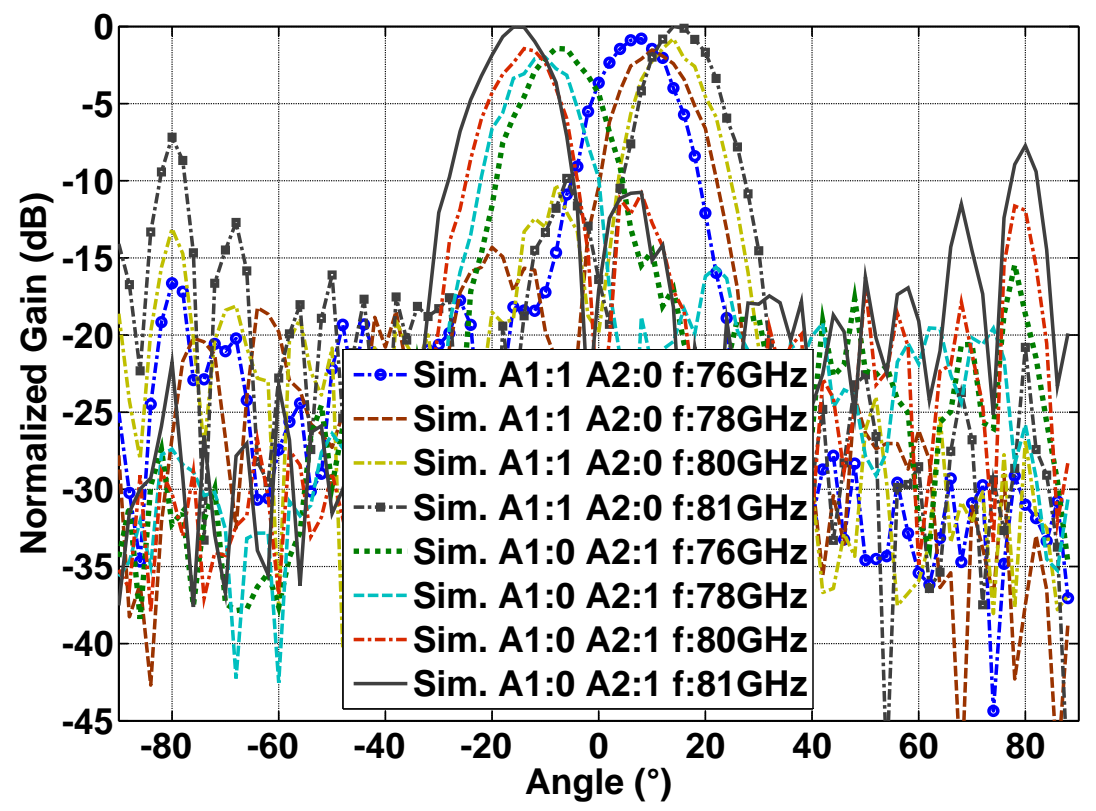

Figure 5.7.: Beam steering in E-plane by change of operating frequency $(76 \mathrm{GHz}$ to $81 \mathrm{GHz})$. 
(a) Normalized E-plane radiation pattern at $78 \mathrm{GHz}$ if signal is supplied by only one port.

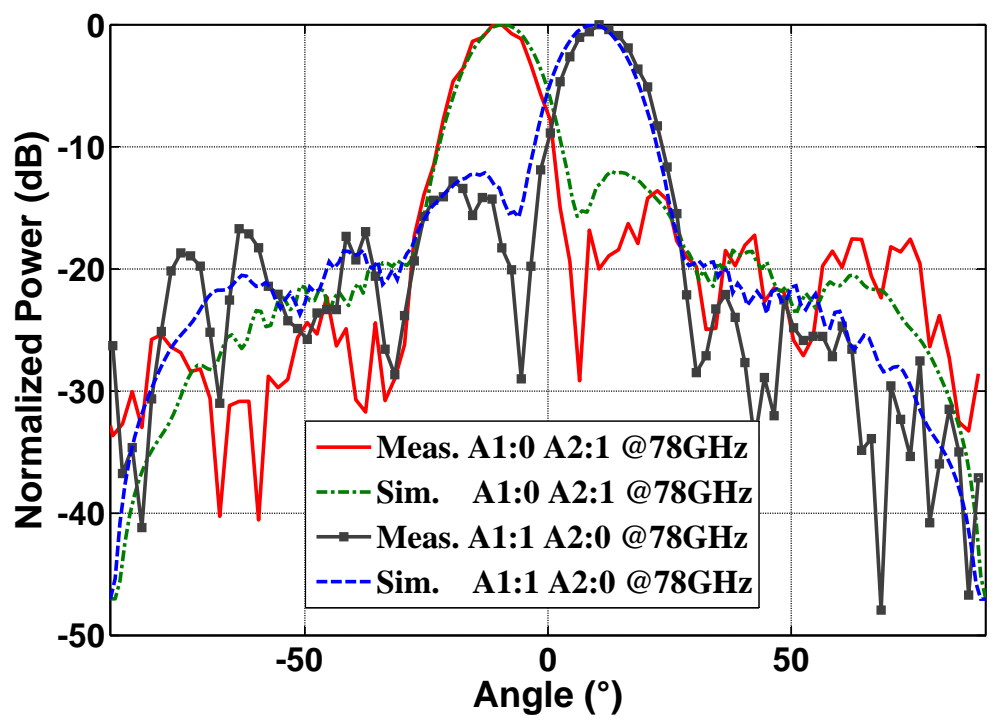

(b) Normalized E-plane radiation pattern at $81 \mathrm{GHz}$ if signal is supplied by only one port.

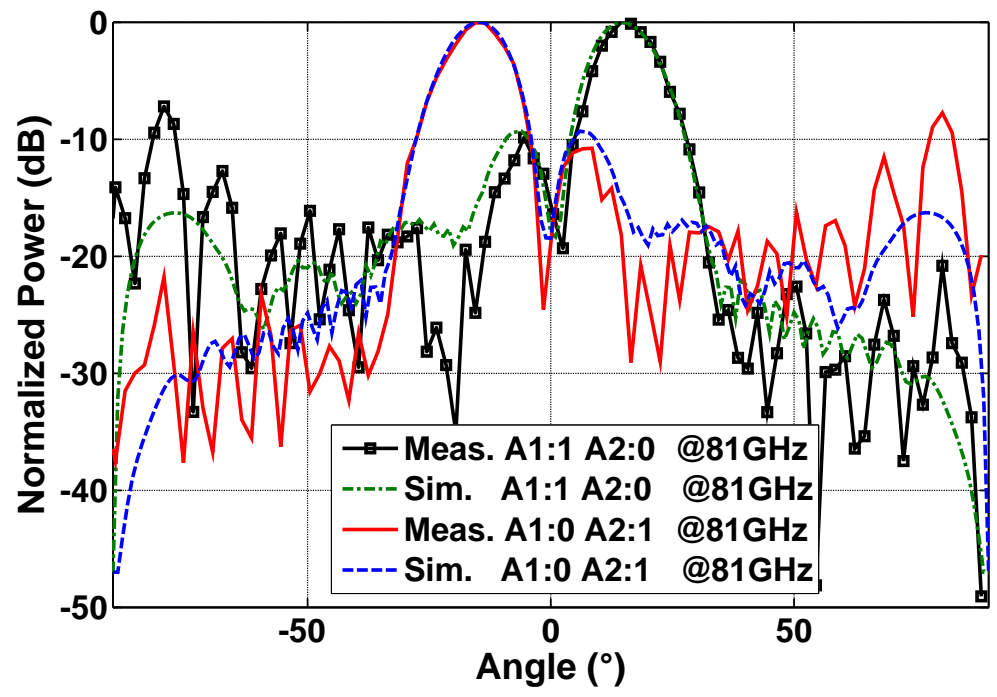

Figure 5.8.: Radiation pattern at different operating frequencies. 


\subsection{Setup based on active MMIC I-Q vector modulator}

\subsubsection{Test configuration}

Fig. 5.9 shows the second antenna setup employing the active I-Q vector modulator MMIC. The advantage of this setup compared to the first one is that antenna measurements are fully automated thanks to the I-Q modulators. The dual-fed antenna is characterized as a transmitter antenna in the desired frequency range since the characterized I-Q vector modulator is in a transmitter path of a transceiver MMIC. This setup is exactly same as the one shown in Fig. 5.1 except for the control mechanism of the I-Q modulator. The I-Q vector modulator is controlled using data acquisition cards (DAQ) to be able to supply the desired phase shift and amplitude change in the transmitter path. LabVIEW is used as a software on a computer for a direct control of the DAQ card.

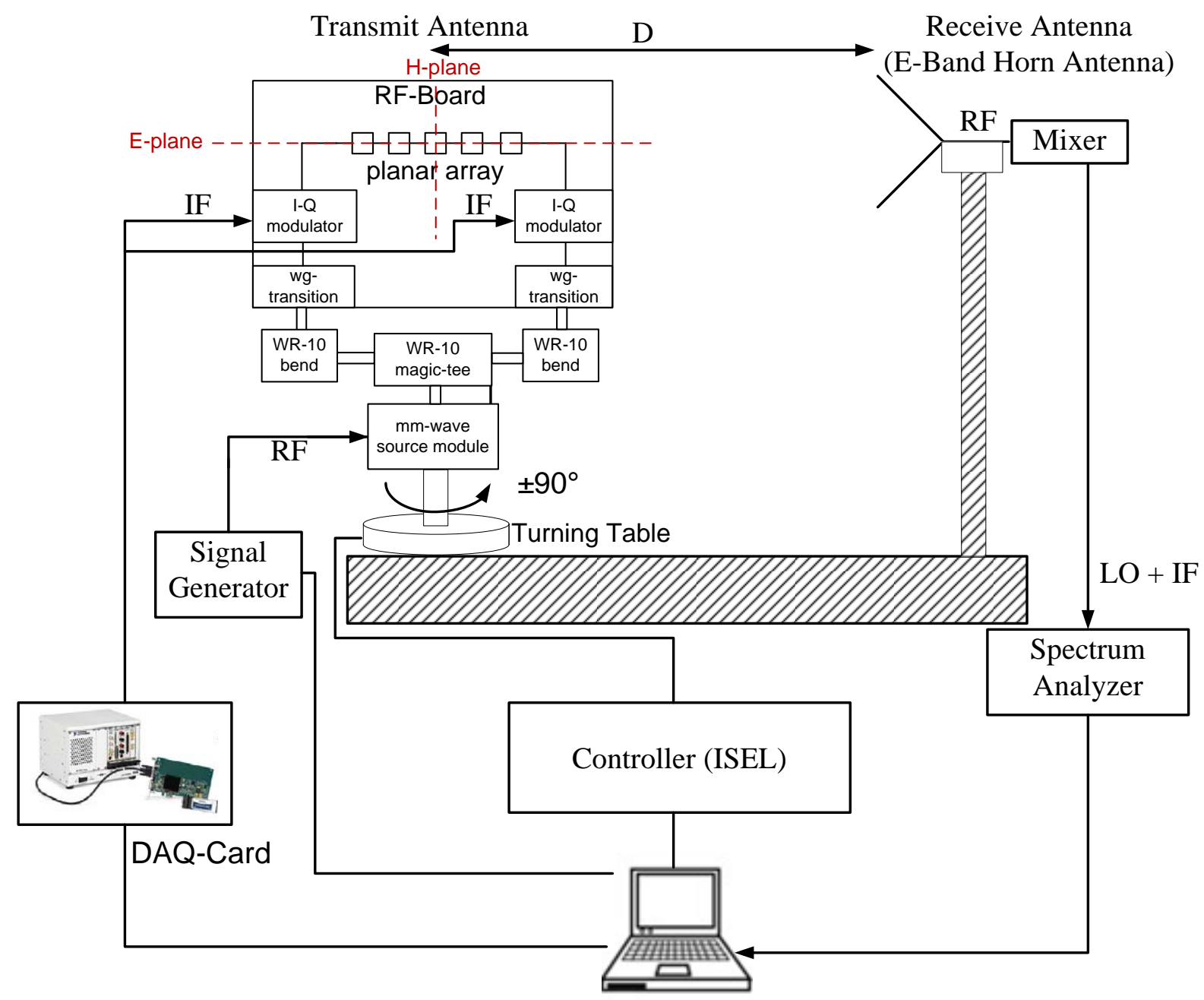

Figure 5.9.: Antenna measurement setup employing bare-die I-Q vector modulator.

The presented antenna concept is based on the use of a single layer printed circuit board. 
In Fig. 5.11, an RF-board is fixed on the measurement platform using some plastic screws. This board houses a linear array antenna and I-Q transceivers which house I-Q modulators. A linear array antenna which is made up of 5 identical patches is shown in Fig. 5.10(a). High impedance feed lines are converted to $50 \Omega$ lines using quarter wave transformers. Fig. 5.10(b) shows the wire-bonded I-Q transceiver chip and planar baluns on the board. As shown in Fig. 5.12, control boards and DAQ hardware are placed at the backside of the platform. More details about control boards and DAQ hardware are given in Section 4.3.

(a) Linear array antenna without amplitude tapering.

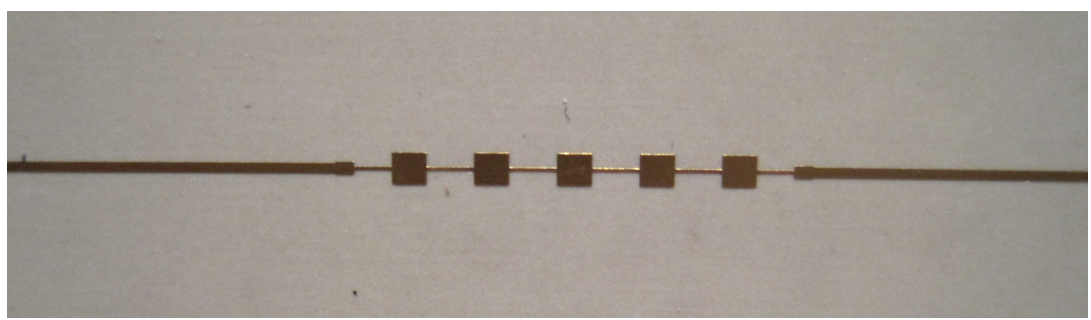

(b) Bare die I-Q transceiver bonded on an RF board.

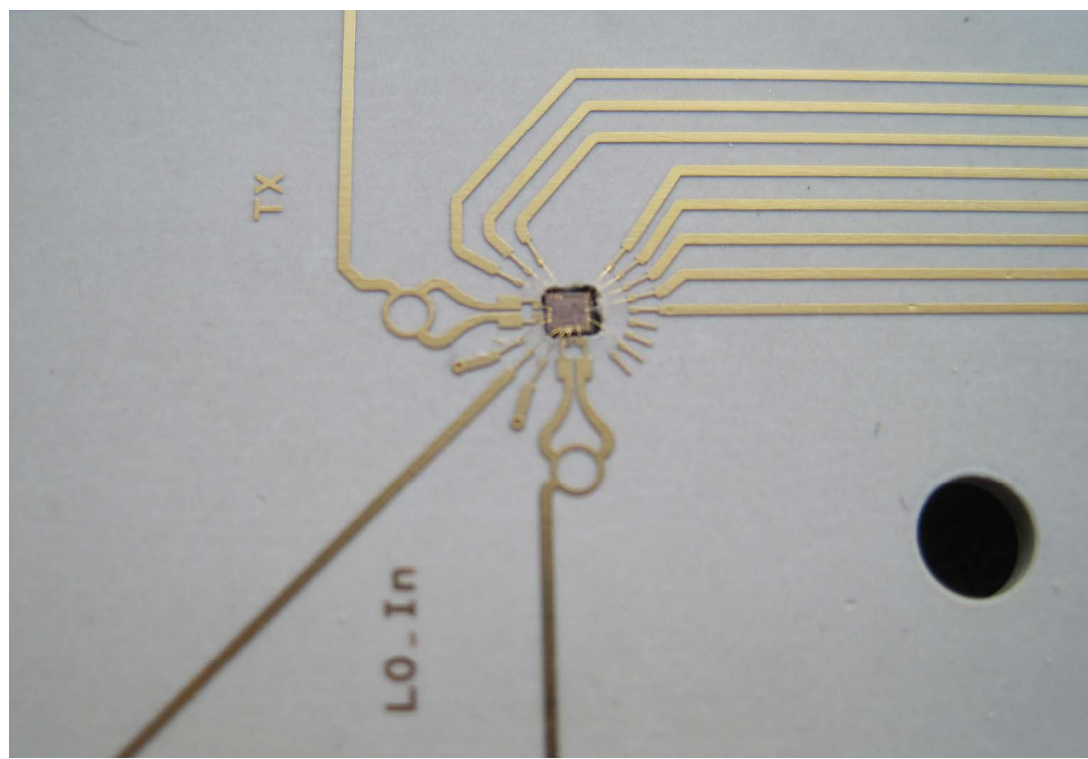

Figure 5.10.: Realized antenna setup.

\subsubsection{Antenna without amplitude tapering}

A linear array antenna, made up of 5 patch elements, is characterized using parameters listed in Table A.1. Table 5.2 shows parameters of the designed antenna, which is plotted in Fig. A.2. Fig. 5.13(a) shows antenna setup including waveguide transitions. Since no amplitude tapering is applied, the patch widths in the array are identical. The waveguide transitions are placed vertical to the E-plane not to disturb the beam steering. Fig. 5.13(b) shows the measured S-parameters using a 2-port network analyzer between $70 \mathrm{GHz}$ and 90 


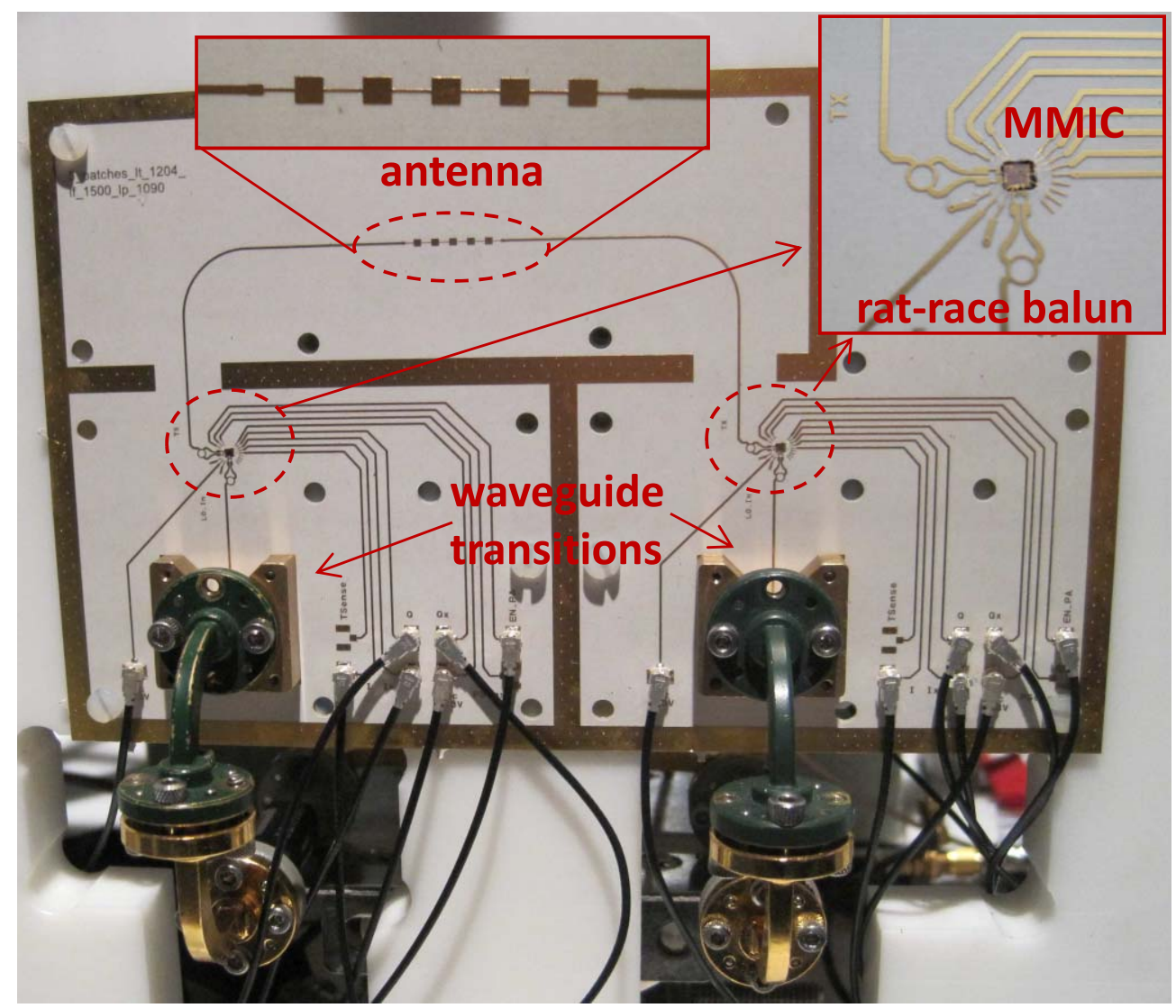

Figure 5.11.: RF board employing a linear array antenna and MMIC phase shifters on the measurement platform.

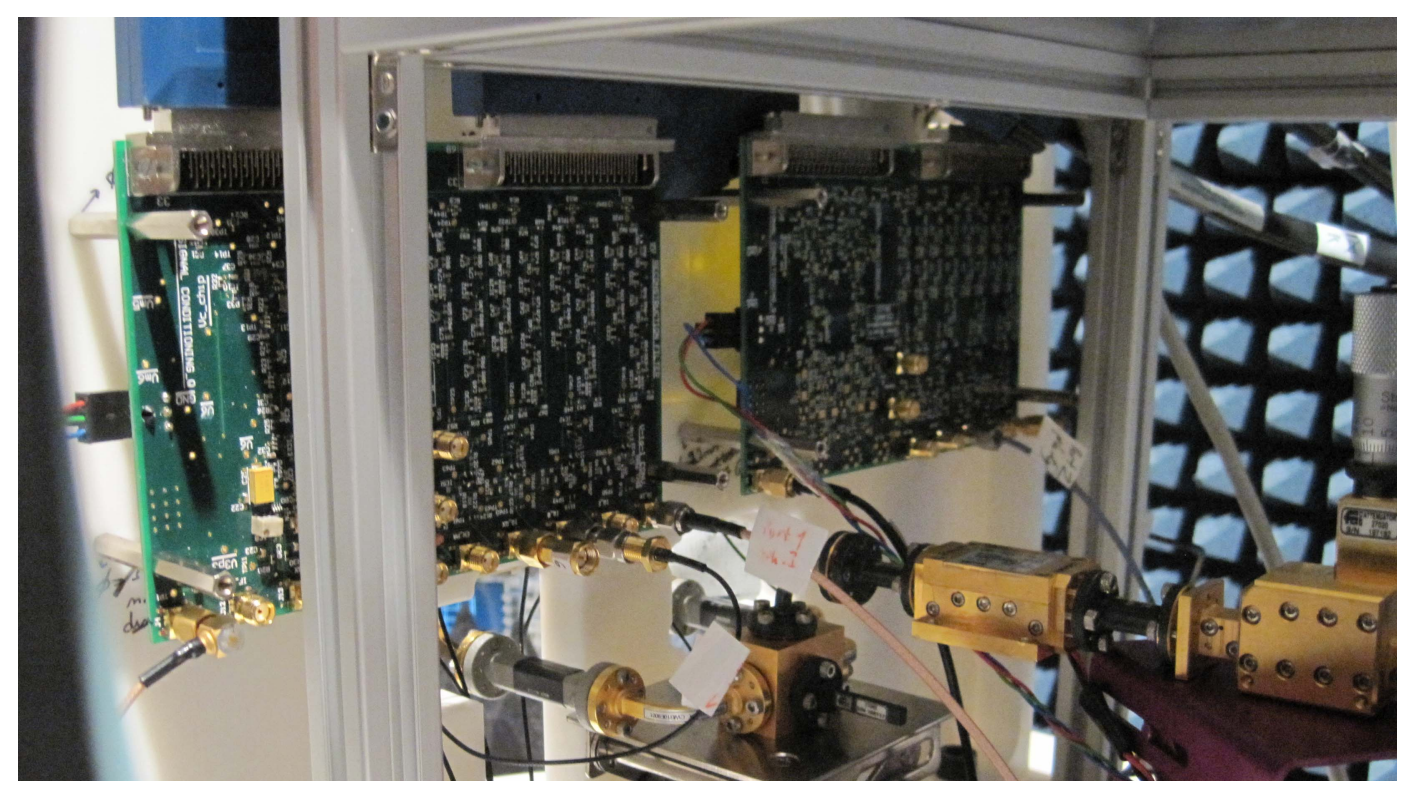

Figure 5.12.: Control boards for I-Q modulators. 
GHz. The return losses in this figure are dominated by the return losses due to waveguide transitions.

Table 5.2.: Parameters of a dual fed array design without amplitude tapering at $77 \mathrm{GHz}$.

\begin{tabular}{c|c}
\hline Design parameter & Value \\
\hline \hline Number of patches $(N)$ & 5 \\
\hline$L_{f}(\mathrm{~mm})$ & 1.5 \\
\hline$L_{p}(\mathrm{~mm})$ & 1.09 \\
\hline$W_{f}(\mathrm{~mm})$ & 0.1 \\
\hline$W_{p}(\mathrm{~mm})$ & 1.02 \\
\hline
\end{tabular}

\subsubsection{Measurement results}

The measurements are performed at the operating frequency of $77 \mathrm{GHz}$ within a field of view of $\pm 40^{\circ}$ with $1^{\circ}$ steps. Fig. 5.14 shows the normalized measured and simulated sum $(\Sigma) /$ difference $(\Delta)$ patterns in the E-plane. The simulations were carried out including the linear array, bends and the transmission lines until the outputs of the MMICs, as shown in Fig. 5.11. The antenna is fed from both sides in the measurements. If the phase difference $\phi_{\mathrm{ps}}$ between both feeds is $180^{\circ}$, the sum pattern is generated looking to the broadside. If the phase difference is $0^{\circ}$, the dual-beam (difference) pattern is generated which looks to the $\pm 10^{\circ}$ directions. The sum pattern is $3 \mathrm{~dB}$ higher than the peaks of the difference pattern. The gain difference between the sum and difference patterns is almost $25 \mathrm{~dB}$ at the broadside. The cross-polarized radiation pattern in the E-plane is better than $-25 \mathrm{~dB}$ at the broadside. The setup can also be used in receive mode, using the IF outputs of the I-Q modulator as receivers. This allows employing monopulse techniques using the sum and difference patterns.

The maximum measured beam steering angle for this antenna configuration is $\pm 8^{\circ}$ in the E-plane, when the amplitude ratio and the phase difference are adjusted to the given values, shown in Fig. 5.15. Normalized measurement and simulation patterns are plotted for $0^{\circ}$ and $\pm 8^{\circ}$ directions. The HPBW of the main beams is $16^{\circ}$ and the measured sidelobe levels are lower than $-15 \mathrm{~dB}$. By setting the amplitude and phase values of the signals of both ports, the direction of the main beam can be steered to any direction within this scan range.

Fig. 5.16 shows measured and simulated H-plane patterns for $A 1=A 2$ and $\Delta \phi=180^{\circ}$. As seen from this figure, measurement and simulation results agree well with each other in the field view of $0^{\circ}$ to $90^{\circ}$. However, measured pattern between $-90^{\circ}$ and $0^{\circ}$ is much lower than the expected pattern. The reason is the WR-10 waveguide bends used for bending signals with 180 degrees between waveguide transitions and other waveguide components in the setup. Signal reflections due to these metallic waveguide components degrade the result at one half of the field view, as expected. Measured cross-polarized pattern of this signal is 
(a) Antenna setup for the characterization.

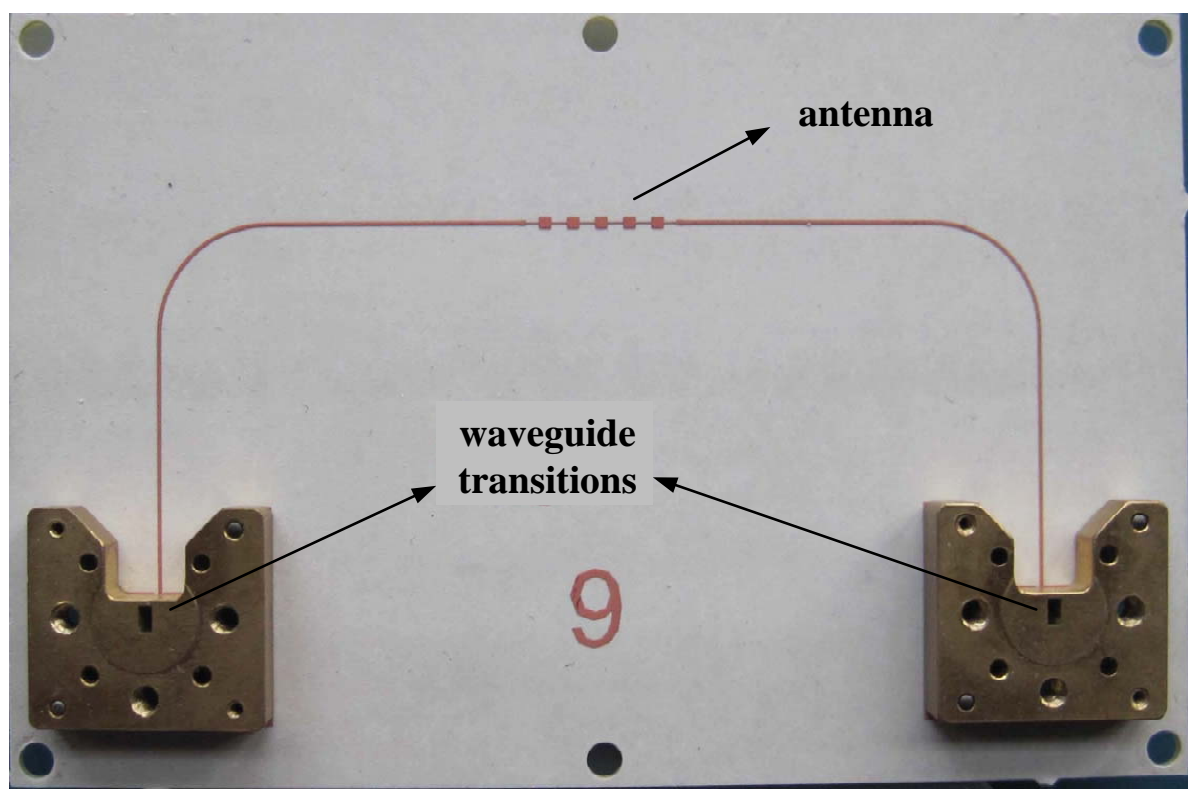

(b) Measured S-parameters of setup including array antenna with 5 patch elements.

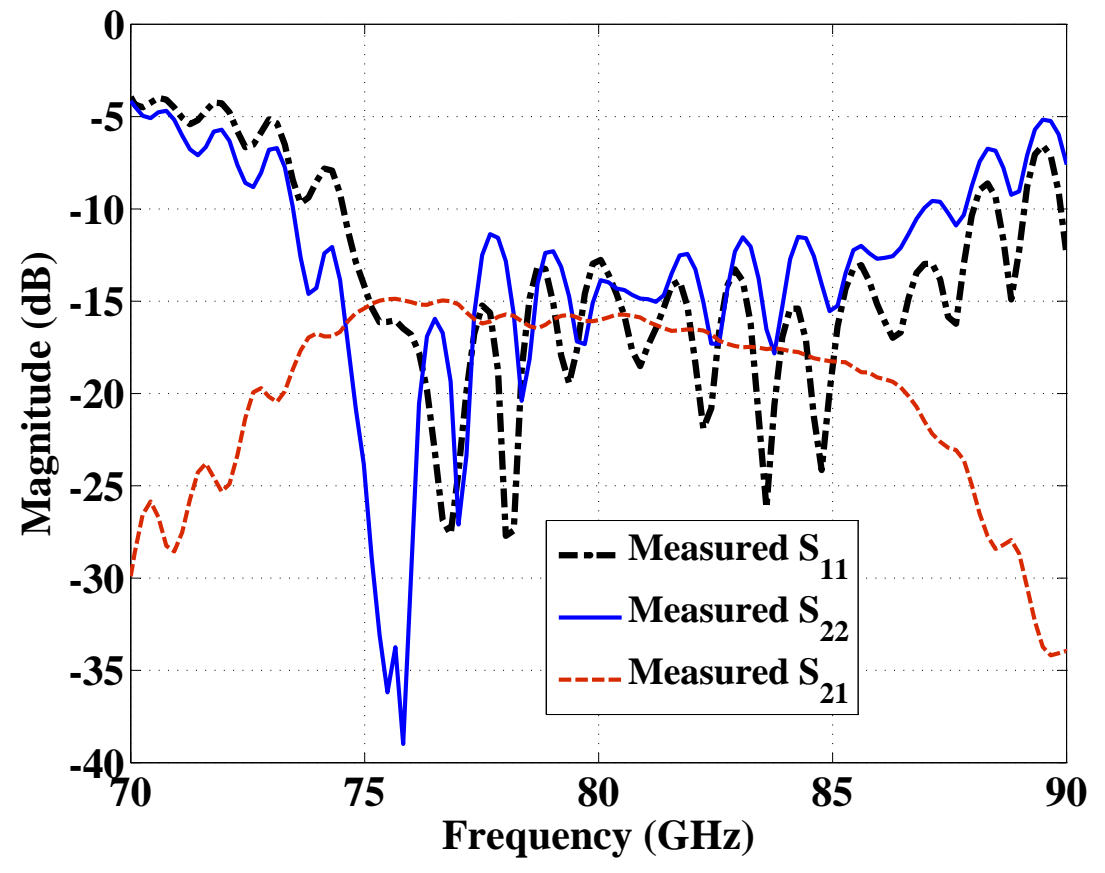

Figure 5.13.: Characterization of the linear array antenna made up of 5 patch elements.

better than $17 \mathrm{~dB}$.

The maximum beam steering angle is $\pm 18^{\circ}$ in the E-plane, when the operating frequency is changed from 75 to $81 \mathrm{GHz}$ as shown in Fig. 5.17. The grating lobes are beginning to appear at $\pm 80^{\circ}$ if the frequency is increased. The normalized beam is steered at $\pm 10^{\circ}$ if one of two 


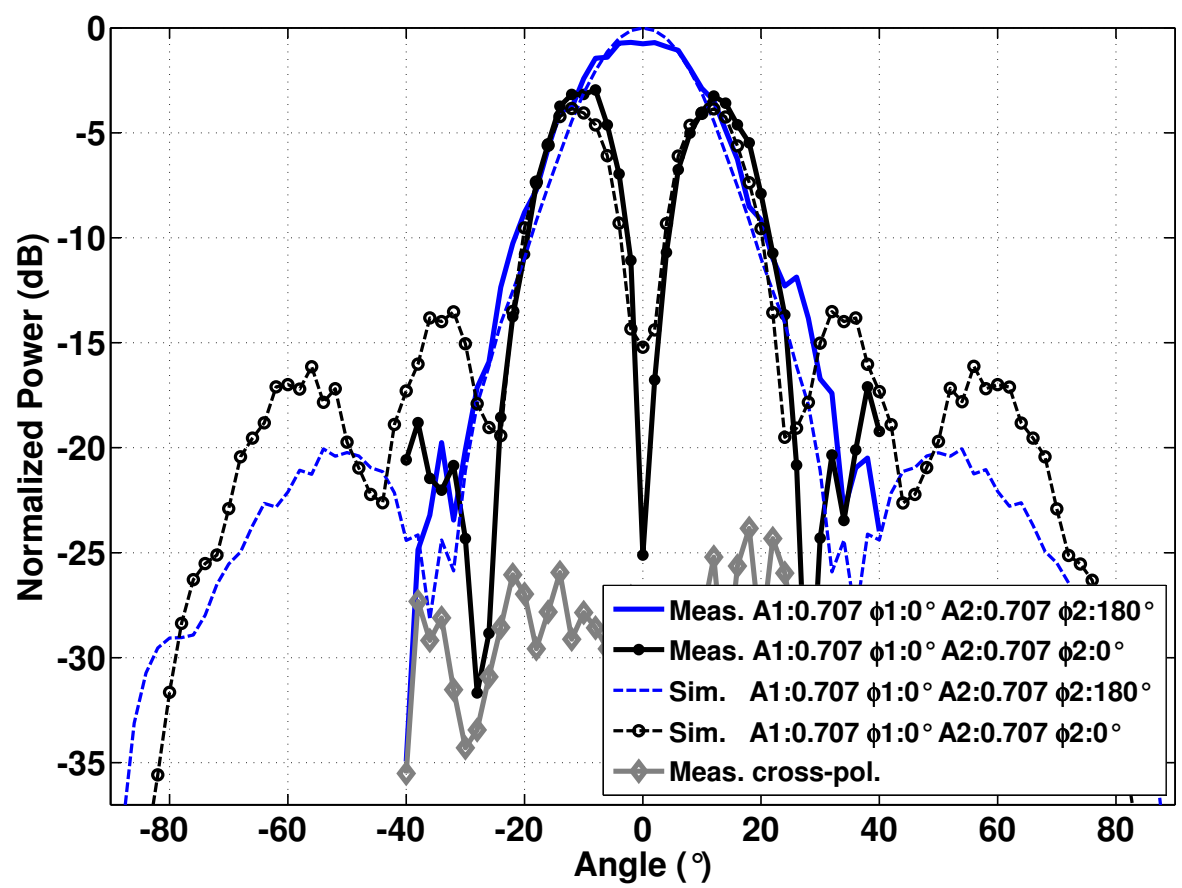

Figure 5.14.: Sum and difference patterns in the E-plane (Measurement \& Simulation).

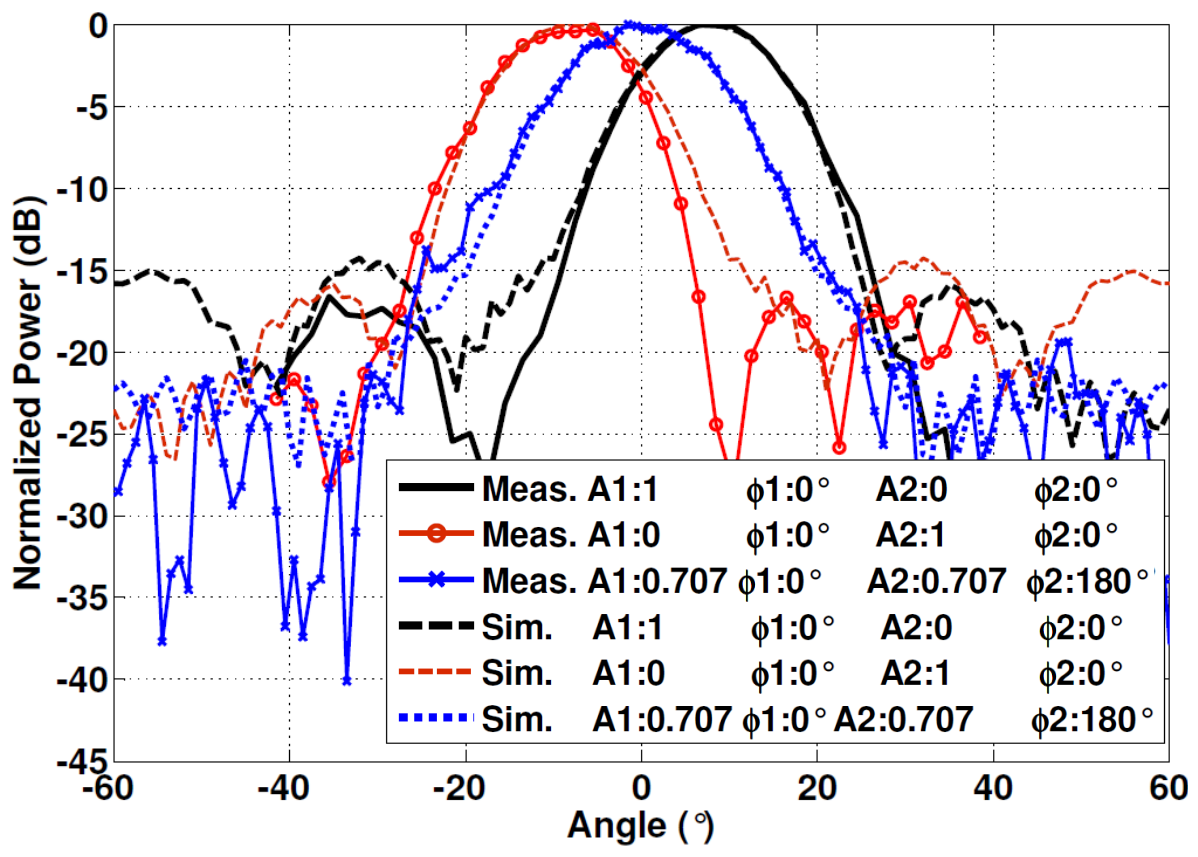

Figure 5.15.: Beam steering in the E-plane (Measurement \& Simulation).

channels are turned off and the operating frequency is $75 \mathrm{GHz}$ as shown in Fig. 5.18(a). The sidelobe levels are higher than $13 \mathrm{~dB}$. Fig. 5.18(b) shows two beams directed at $\pm 18^{\circ}$ if the frequency is $81 \mathrm{GHz}$. In this figure, it can be clearly seen that grating lobes appear within the field of view. Since increase in frequency also causes increase in electrical length between 


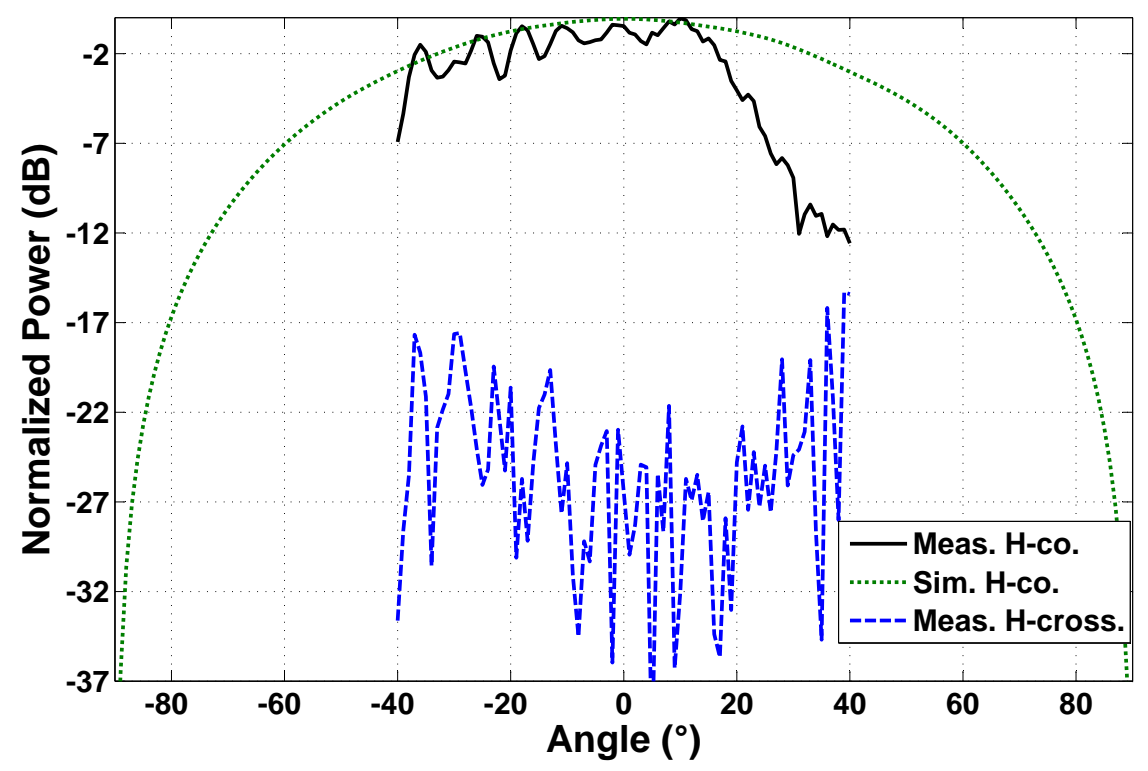

Figure 5.16.: H-plane radiation pattern.

subsequent patches in the setup, these unwanted lobes also appear inside the field.

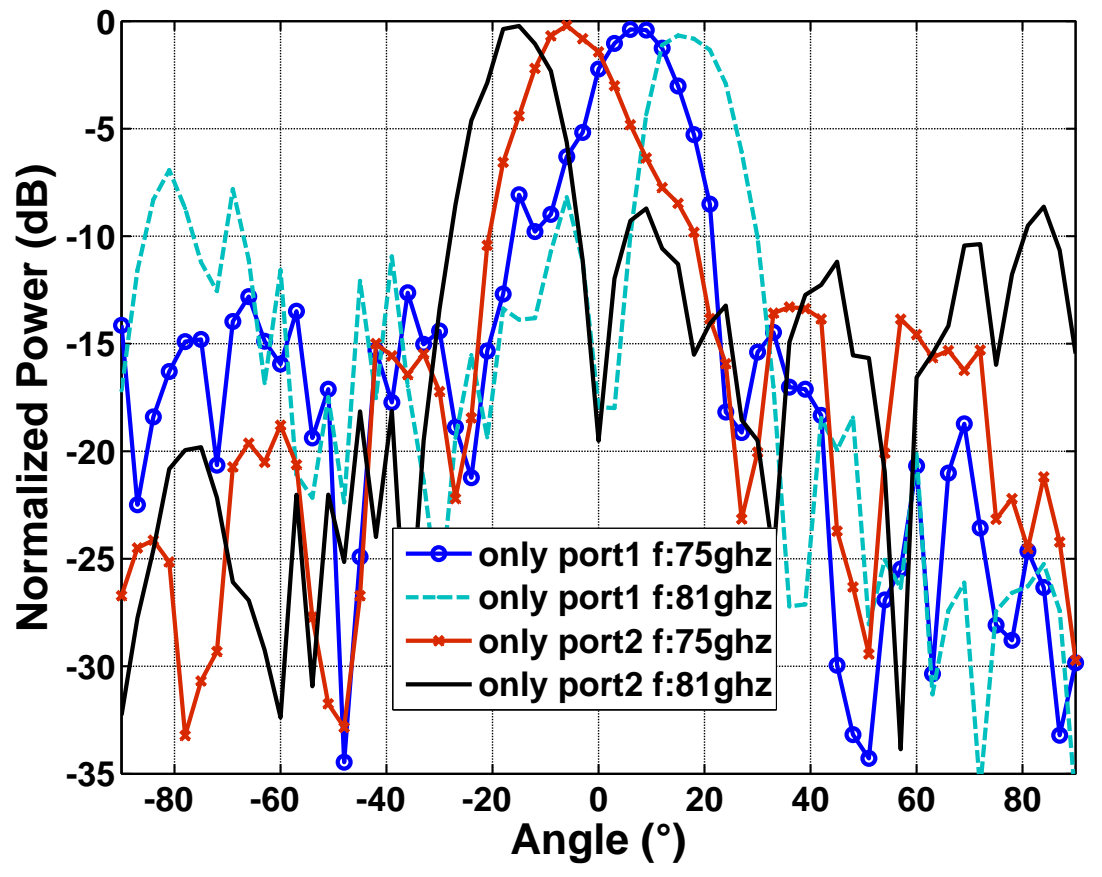

Figure 5.17.: Beam steering by change of operating frequency ( $\mathrm{f}=75$ to $81 \mathrm{GHz}$ ).

Since the active wire-bonded I-Q modulators have been used, the antenna gain could not be measured properly. However, since the received power using a $20 \mathrm{~dB}$ horn antenna at the receiver side, the distance between two antennas $(1 \mathrm{~m})$, the free space attenuation $(70.1 \mathrm{~dB}$ 
(a) Normalized E-plane radiation pattern at $75 \mathrm{GHz}$ if signal is supplied through only one port.

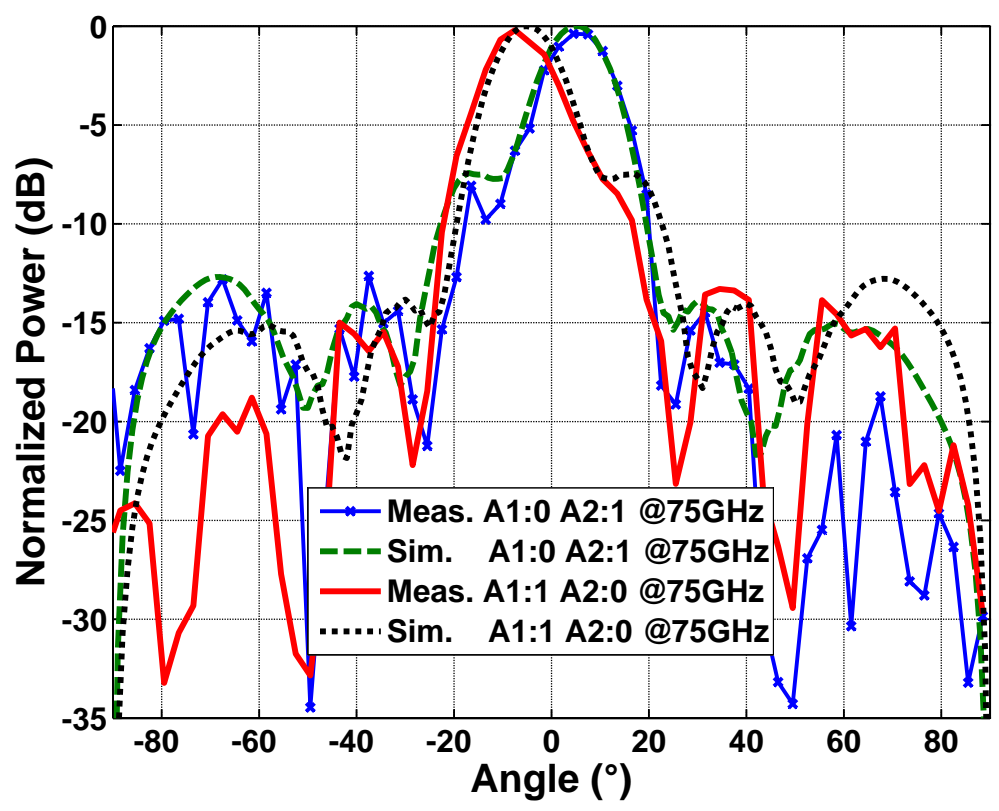

(b) Normalized E-plane radiation pattern at $81 \mathrm{GHz}$ if signal is supplied through only one port.

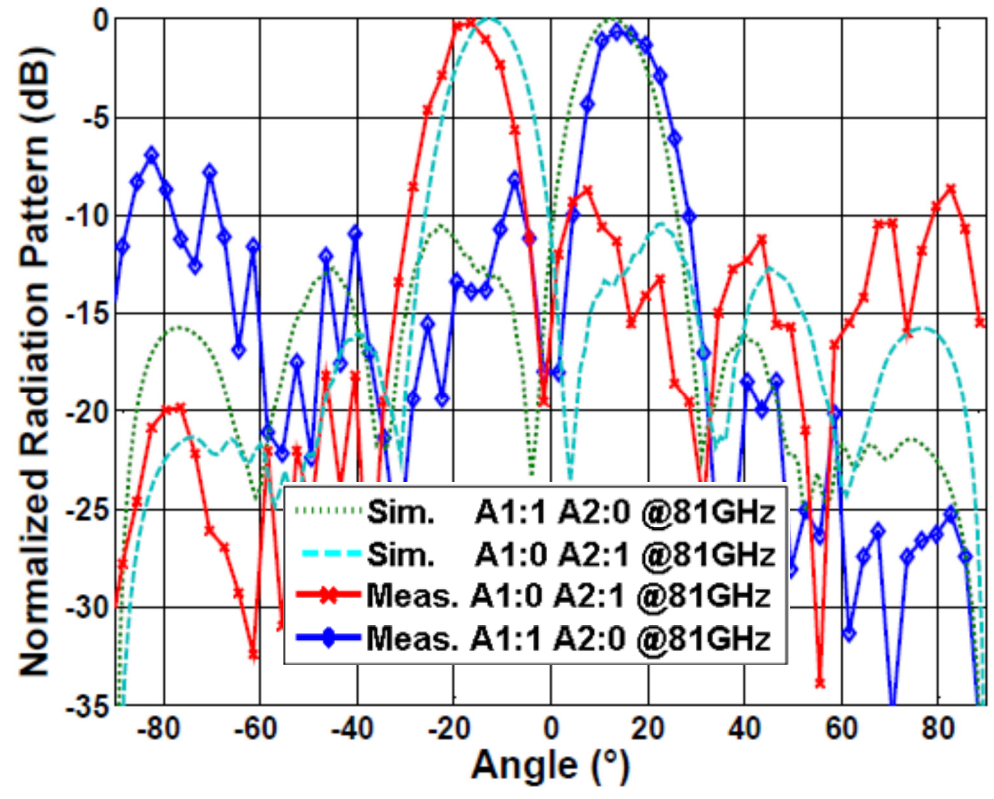

Figure 5.18.: Radiation pattern at different frequencies.

at $77 \mathrm{GHz}$ for $1 \mathrm{~m}$ distance), the output powers of the I-Q transceiver MMICs ( $2 \mathrm{dBm})$, and the transmission line losses between the MMICs and the linear array antenna $(8.1 \mathrm{dBm})$ are known, the measured gain was calculated as $9.6 \mathrm{dBi}$ using the Friis transmission equation. Considering the uncertainty of the gain estimation, this fits well to the simulated gain of 9.9 dBi. 
Based on the measurement and simulation results shown in Fig. 5.5 and Fig. 5.15, the two different linear arrays with different antenna parameters (The first antenna has $N=6, L_{f}=1.41$ $\mathrm{mm}$ and amplitude tapering, whereas the second antenna has $N=5, L_{f}=1.5 \mathrm{~mm}$ and no amplitude tapering) enable the same maximum beam steering angle of $16^{\circ}$. Although, the first antenna is expected to have more limited beam steering than the second one due to the number of patch antennas and the shorter feed line lengths, the amplitude tapering contributes an increase in the maximum beam steering range.

After analysis of the proposed concept, a comparison between the proposed phased array and other dual-fed phased arrays in the literature is given in Table 5.3. The proposed array achieves a narrower scan range compared to the designs, shown in Table 5.3. However, the setup presented here requires just 2 phase shifters and the achieved scan range can fulfill the applications, as explained in Chapter 1. This work shows a gain variation of just $0.2 \mathrm{~dB}$ in the entire scan range. Moreover, the proposed antenna array has a much lower sidelobe level. The proposed phased array architecture can also be used as a monopulse antenna.

Table 5.3.: Comparison between the dual-fed phased array architectures.

\begin{tabular}{l|l|l|l|l}
\hline Parameters & {$[\mathbf{2 3}]$} & {$[\mathbf{2 4}]$} & {$[\mathbf{7 1}]$} & $\begin{array}{l}\text { This } \\
\text { work }\end{array}$ \\
\hline \hline Number of antenna elements & 5 & 8 & 4 & 5 \\
\hline Number of phase shifters & 4 & 2 & 4 & $\begin{array}{l}2 \text { I-Q } \\
\text { mod. }\end{array}$ \\
\hline Number of gain amplifiers & - & 8 & - & - \\
\hline Center frequency (GHz) & 5.8 & 2 & 2 & 77 \\
\hline Max. sidelobe levels & $-10 \mathrm{~dB}$ & $-10 \mathrm{~dB}$ & $-9 \mathrm{~dB}$ & $-15 \mathrm{~dB}$ \\
\hline HPBW & N.A. & N.A. & $24-28^{\circ}$ & $16^{\circ}$ \\
\hline Beam scan range & $22^{\circ}$ & $25^{\circ}$ & $20^{\circ}$ & $16^{\circ}$ \\
\hline Max. gain (dBi) & 11.3 & N.A. & 8.7 & 9.6 \\
\hline $\begin{array}{l}\text { Gain variation within scan } \\
\text { range }\end{array}$ & $0.4 \mathrm{~dB}$ & N.A. & $1.8 \mathrm{~dB}$ & $0.2 \mathrm{~dB}$ \\
\hline Monopulse antenna & no & no & no & yes \\
\hline
\end{tabular}




\section{Conclusion and Future Work}

\subsection{Summary and Conclusion}

In this dissertation, a new dual-fed phased array has been developed, fabricated and characterized at $77 \mathrm{GHz}$ to enable a low-cost and less-complex electronic beam scanning solution since only a single phase shifter and two variable gain attenuators are used for a complete array with any number of antenna elements. Therefore, this solution can be also used for commercial applications such as sensors and communication systems.

By changing the amplitude ratio and the phase difference of the two signals supplied through opposite feeds, a beam can be steered to different directions. However, increasing the number of radiating elements in the array limits the maximum scan range. As shown in Table 2.2 , via this concept, a microstrip array with 4 patch elements has a maximum scan range of $32^{\circ}$, whereas an array with 15 patch elements enables only $12^{\circ}$ scan range. Table 2.3 shows that an array with an element spacing of $\lambda_{g} / 2$ has no beam steering capability. However, the same array with an element spacing larger or smaller than $\lambda_{g} / 2$ can be used for beam steering.

Because of its topology, the new concept is highly robust against phase and amplitude errors in the RF paths. An antenna array is simulated in Section 2.2.2 to show effects of amplitude and phase errors on the overall pattern. Even $\pm 30^{\circ}$ phase error or $\pm 1.94 \mathrm{~dB}$ amplitude error result in almost no change in the direction and HPBW of the main beam. Since only two RF paths exist and are located on both sides of the antenna array, channel mismatch and crosstalk aren't an issue.

In addition to its beam steering capability, the proposed concept enables sum and difference patterns if two signals with the same amplitude and $0^{\circ} / 180^{\circ}$ phase differences are fed to an array. In Chapter 5, measured and simulated sum and difference patterns are shown for both measurement setups. A difference pattern can be used to obtain a null beam with a dynamic range higher than 30-35 $\mathrm{dB}$ in the broadside direction, whereas a sum pattern looking into the broadside direction with very low sidelobe levels can be obtained by adjusting a $180^{\circ}$ phase difference between both supplied signals. Null beams can be directed to desired directions without affecting the direction and shape of the main beam if the phase difference of the supplied signals is varied, as explained in Section 2.2.2.

In Chapter 1, conventional phased arrays were presented briefly. In this chapter, the advantages of the conventional phased arrays were listed. Later on, their potential challenges were described which avoid use of phased arrays for commercial applications. At the end of the 
chapter, a novel beam scanning approach was presented which eliminates the disadvantages of the conventional phased arrays.

In Chapter 2, the novel phased array concept was explained in detail. In order to understand its working principle, first the traveling wave antenna concept was introduced. Then, architecture, working principle and pattern capabilities of the new concept were presented thoroughly.

In Chapter 3, an analytical antenna model was developed for the proposed concept and verified in MATLAB using closed form expressions. This model was optimized assuming that the antenna structure is printed on a dielectric substrate (Table A.3) within the desired frequency range of $75 \mathrm{GHz}$ and $85 \mathrm{GHz}$. Finally, the effects of each design parameter of a linear array on the overall pattern were investigated.

In Chapter 4, mm-wave components, required for realizing a dual-fed phased array measurement setup at $77 \mathrm{GHz}$, were characterized. In Section 4.3, a $77 \mathrm{GHz}$ transceiver MMIC which houses a vector I-Q modulator in its transmitter path, was characterized. Since the I-Q transceiver MMIC has a fully differential configuration, a planar mm-wave balun is analyzed to convert differential signals to single-ended ones since there are no measurement devices with differential inputs and outputs in the laboratory. Since a mm-wave signal is generated by a signal generator with a waveguide output, a WR-10 waveguide to microstrip line transition had to be designed to deliver mm-wave signals to a microstrip linear array. Since the aim is to have a low cost and broadband transition, the transition was fabricated on a single layer dielectric substrate using a standard photolithographic process. This new transition allows the integration of multi-channel array systems with an element spacing of $\lambda_{0} / 2$ or less thanks to the feeding technique between microstrip line and patch antenna (Microstrip line is fed through H-plane of waveguide). The measurement results show that the transition with the waveguide iris has a bandwidth of $12 \mathrm{GHz}(15 \%)$ at a return loss below $-10 \mathrm{~dB}$ and a minimum insertion loss of $0.6 \mathrm{~dB}$ at the design frequency of $77 \mathrm{GHz}$.

After the characterization of the required mm-wave components, two different dual-fed phased array test setups employing a linear array antenna were designed and built in Chapter 5 to synthesize desired antenna characteristics. The phase shifting and amplitude changing were performed using commercial passive WR-10 waveguide phase shifter and variable attenuator in the first setup. In this setup, a linear array antenna employing 6 patch elements (including amplitude tapering) was characterized. In the second setup, an I-Q vector modulator was used for changing phase and amplitude values in the transmit paths. In this setup, a linear array antenna employing 5 patch elements (without amplitude tapering) was measured. The sum / difference antenna patterns, beam steering by using phase shifter \& attenuator and beam steering by change of the operating frequency from $75 \mathrm{GHz}$ to $81 \mathrm{GHz}$ were simulated and measured for both measurement setups. In addition, the H-plane co- and cross-polarized antenna patterns are shown. The measurement results agree well with the full-wave simulation results. These results prove the functionality of the proposed concept. 
After the analysis of the proposed concept, a comparison between a conventional phased array and the dual-fed phased array concept is given in Table 6.1. It has been analyzed in Section 2.2.2 that the proposed concept is highly robust against phase and amplitude errors. However, since the progressive phase shift between the patch elements is controlled via element spacing in the new approach, the maximum field of view is more limited. An increase in the number of elements makes field of view even much narrower. Compared to the conventional arrays where one phase shifter and one variable gain amplifier are required for each channel, only one phase shifter and 2 variable gain amplifiers are required for the whole linear array, independent of the number of radiating elements. Electronic beam scanning is possible by using a phase shifter in a conventional phased array, whereas this is only possible by using phase shifter and variable attenuator in the proposed approach. Since the number of RF elements and their control circuits increase the manufacturing complexity, the proposed concept is very simple. Low sidelobe levels can be obtained in both approaches. At the conventional one, this is possible by using variable gain amplifiers. At the proposed design, this is only possible by changing widths of patch elements.

Table 6.1.: Comparison between the conventional and dual-fed phased array architectures.

\begin{tabular}{l|l|l}
\hline Parameters & $\begin{array}{l}\text { Conventional } \\
\text { phased array }\end{array}$ & $\begin{array}{l}\text { Novel dual-fed } \\
\text { phased array }\end{array}$ \\
\hline \hline $\begin{array}{l}\text { Robustness against phase and } \\
\text { amplitude errors }\end{array}$ & low & high \\
\hline Field of view & large & narrower \\
\hline Number of RF components & high & low \\
\hline Necessity of array calibration & yes & no \\
\hline $\begin{array}{l}\text { Beam scanning via change of } \\
\text { frequency }\end{array}$ & no & yes \\
\hline Manufacturing complexity & hard & easy \\
\hline Monopulse antenna & no & yes \\
\hline Low sidelobes & yes & yes \\
\hline
\end{tabular}

\subsection{Future Work}

The implemented setups performed well and demonstrated beam scanning capability of the proposed concept. Some recommendations for the future work are:

- In this thesis, a microstrip linear array, as shown in Fig. 2.12, was used for characterization. However, losses due to long feed lines on both sides of the array and between patch elements cause a degradation in the antenna efficiency. Instead, in the future work, a linear slotted waveguide array, which is shown in Fig. 2.13, can be used in the setup to improve the antenna efficiency of the novel concept. 
- In this work, two setups with element spacings larger than $\lambda_{g} / 2$ were characterized. In a future design, an array with element spacings smaller than $\lambda_{g} / 2$ can be characterized to show the wide beam scan range capability of the proposed concept experimentally, up to $38^{\circ}$.

- In the measured setups, the H-plane antenna patterns were distorted by the WR-10 waveguide bends. In a future design, a new waveguide to microstrip transition, which transfers the signal to the back side of the board, can be employed to eliminate the waveguide bends. Additionally, a planar array antenna, as shown in Fig. 2.14, can be designed to obtain a sharper beam in azimuth and elevation. 


\section{Bibliography}

[1] D. Parker and D. Zimmermann, "Phased Arrays - Part 1: Theory and Architectures," IEEE Transactions on Microwave Theory and Techniques, vol. 50, no. 3, pp. 678 -687, Mar. 2002.

[2] — - "Phased Arrays- Part 2: Implementations, Applications, and Future Trends," IEEE Transactions on Microwave Theory and Techniques, vol. 50, no. 3, pp. 688 -698, Mar. 2002.

[3] D. Liu, U. Pfeiffer, J. Grzyb, and B. Gaucher, Advanced Millimeter-wave Technologies: Antennas, Packaging and Circuits. Wiley, 2009.

[4] R. Emrick, S. Franson, J. Holmes, B. Bosco, and S. Rockwell, "Technology for emerging commercial applications at millimeter-wave frequencies," in IEEE/ACES International Conference on Wireless Communications and Applied Computational Electromagnetics, April 2005, pp. 425 - 429.

[5] J. Hasch, E. Topak, R. Schnabel, T. Zwick, R. Weigel, and C. Waldschmidt, "MillimeterWave Technology for Automotive Radar Sensors in the $77 \mathrm{GHz}$ Frequency Band," IEEE Transactions on Microwave Theory and Techniques, vol. 60, no. 3, pp. 845 -860, Mar. 2012.

[6] J. Wenger, "Automotive radar - status and perspectives," in IEEE Compound Semiconductor Integrated Circuit Symposium, Oct. 2005, pp. 21 - 24.

[7] D. Ehyaie, Novel Approaches to the Design of Phased Array Antennas: Low Cost, Low Complexity Phased Array Antenna Design. LAP Lambert Academic Publishing, 2012.

[8] I. Sarkas, M. Khanpour, A. Tomkins, P. Chevalier, P. Garcia, and S. Voinigescu, "Wband 65-nm CMOS and SiGe BiCMOS transmitter and receiver with lumped I-Q phase shifters," in IEEE Radio Frequency Integrated Circuits (RFIC) Symp., Jun. 2009, pp. 441-444.

[9] C. Wagner, M. Hartmann, A. Stelzer, and H. Jaeger, "A Fully Differential 77-GHz Active IQ Modulator in a Silicon-Germanium Technology," IEEE Microwave and Wireless Components Letters, vol. 18, no. 5, pp. 362 -364, May 2008.

[10] A. Natarajan, A. Komijani, and A. Hajimiri, "A fully integrated 24-GHz phased-array transmitter in CMOS," IEEE Journal of Solid-State Circuits, vol. 40, no. 12, pp. 25022514, 2005. 
[11] A. M. Niknejad and H. Hashemi, mm-Wave Silicon Technology: 60 GHz and Beyond. Springer, 2007.

[12] G. Rebeiz and K. Koh, "Silicon RFICs for phased arrays," IEEE Microwave Magazine, vol. 10, no. 3, pp. 96-103, May 2009.

[13] A. Natarajan, A. Komijani, X. Guan, A. Babakhani, and A. Hajimiri, "A 77-GHz Phased-Array Transceiver With On-Chip Antennas in Silicon: Transmitter and Local LO-Path Phase Shifting," IEEE Journal of Solid-State Circuits, vol. 41, pp. 2807-2819, Dec. 2006.

[14] A. Babakhani, X. Guan, A. Komijani, A. Natarajan, and A. Hajimiri, "A 77-GHz Phased-Array Transceiver With On-Chip Antennas in Silicon: Receiver and Antennas ," IEEE Journal of Solid-State Circuits, vol. 41, pp. 2795-2806, Dec. 2006.

[15] S. Raman, N. Barker, and G. Rebeiz, "A W-band dielectric-lens-based integrated monopulse radar receiver," IEEE Transactions on Microwave Theory and Techniques, vol. 46, pp. 2308-2316, Dec. 1998.

[16] D. Parker and D. Zimmermann, "Phased arrays-part II: implementations, applications, and future trends," IEEE Transactions on Microwave Theory and Techniques, vol. 50, no. 3, pp. $688-698$, Mar. 2002.

[17] M. Wojnowski, R. Lachner, J. Bock, C. Wagner, F. Starzer, G. Sommer, K. Pressel, and R. Weigel, "Embedded wafer level ball grid array (ewlb) technology for millimeter-wave applications," in 13th IEEE Electronics Packaging Technology Conference (EPTC), 2011, pp. 423-429.

[18] S. Trotta, M. Wintermantel, J. Dixon, U. Moeller, R. Jammers, T. Hauck, A. Samulak, B. Dehlink, K. Shun-Meen, H. Li, A. Ghazinour, Y. Yin, S. Pacheco, R. Reuter, S. Majied, D. Moline, T. Aaron, V. Trivedi, D. Morgan, and J. John, “An RCP Packaged Transceiver Chipset for Automotive LRR and SRR Systems in SiGe BiCMOS Technology," IEEE Transactions on Microwave Theory and Techniques, vol. 60, no. 3, pp. 778-794, 2012.

[19] A. Natarajan, B. Floyd, and A. Hajimiri, "A Bidirectional RF-Combining 60GHz Phased-Array Front-End," in IEEE International Solid-State Circuits Conference, Feb. 2007, pp. $202-597$.

[20] S. Y. Kim, O. Inac, C.-Y. Kim, and G. M. Rebeiz, “A 76-84 GHz 16-element phased array receiver with a chip-level built-in-self-test system," in IEEE Radio Frequency Integrated Circuits Symposium (RFIC), June 2012, pp. 127 -130.

[21] K.-J. Koh, J. May, and G. Rebeiz, "A Q-band (40 -45 GHz) 16-element phased-array transmitter in 0.18-um SiGe BiCMOS technology," in IEEE Radio Frequency Integrated Circuits Symposium, April 2008, pp. 225 -228. 
[22] S. Y. Kim, O. Inac, C.-Y. Kim, and G. Rebeiz, “A 76-84 GHz 16-element phased array receiver with a chip-level built-in-self-test system," in IEEE Radio Frequency Integrated Circuits Symposium (RFIC), June 2012, pp. $127-130$.

[23] E. Ojefors, S. Cheng, K. From, I. Skarin, P. Hallbjorner, and A. Rydberg, "Electrically steerable single-layer microstrip traveling wave antenna with varactor diode based phase shifters," IEEE Transactions on Antennas and Propagation, vol. 55, no. 9, pp. 24512460, 2007.

[24] D. Ehyaie and A. Mortazawi, "A new approach to design low cost, low complexity phased arrays," in IEEE MTT-S International Microwave Symposium Digest, 2010, pp. 1270-1273.

[25] S.-G. Kim and K. Chang, "Low-cost monopulse antenna using bi-directionally-fed microstrip patch array,” Electronics Letters, vol. 39, no. 20, pp. 1428 - 1429, Oct. 2003.

[26] C. Ling and G. Rebeiz, "94 ghz integrated horn monopulse antennas," IEEE Transactions on Antennas and Propagation, vol. 40, no. 8, pp. 981 -984, Aug. 1992.

[27] C. A. Balanis, Antenna Theory Analysis and Design, 2nd ed. Wiley-Interscience, 2005.

[28] M. Skolnik, Introduction to Radar Systems. McGraw-Hill, 1962.

[29] J. Schoebel, T. Buck, M. Reimann, M. Ulm, M. Schneider, A. Jourdain, G. Carchon, and H. Tilmans, "Design considerations and technology assessment of phased-array antenna systems with RF MEMS for automotive radar applications," IEEE Transactions on Microwave Theory and Techniques, vol. 53, no. 6, pp. 1968 - 1975, June 2005.

[30] “RO3003 Laminate Data Sheet,” Rogers Corporation, 100 S. Roosevelt Avenue, Chandler, AZ 85226.

[31] C. Charles, A Calibrated Phase and Amplitude Control System for Phased-array Transmitters. University of Washington, 2006.

[32] M. Dawoud, A. Tennant, and A. Anderson, "Null steering in adaptive arrays using a genetic algorithm," in 24th European Microwave Conference, vol. 2, Sept. 1994, pp. $1108-1114$.

[33] M. Khan and V. Tuzlukov, "Null steering beamforming for wireless communication system using genetic algorithm," in IEEE International Conference on Microwave Technology Computational Electromagnetics, May 2011, pp. 289 -292.

[34] P. Bhartia, R. S. Thomar, and K. V. S. Rao, Millimeter-Wave Microstrip and Printed Circuit Antennas. Artech House Inc, Dec. 1990.

[35] D. Moris, “Sorting Through EM Simulators,” Microwaves \& RF Magazine, 2012. 
[36] T. Metzler, "Microstrip series arrays," IEEE Transactions on Antennas and Propagation, vol. 29, no. 1, pp. 174 - 178, Jan. 1981.

[37] B. Jones, F. Chow, and A. Seeto, "The synthesis of shaped patterns with series-fed microstrip patch arrays," IEEE Transactions on Antennas and Propagation, vol. 30, no. 6, pp. 1206 - 1212, Nov. 1982.

[38] I. J. Bahl and D. K. Trivedi, “A Designer's Guide to Microstrip Line,” Microwaves, vol. 16, pp. 174 - 182, May 1977.

[39] E. O. Hammerstad, "Equations for Microstrip Circuit Design," in 5th European Microwave Conference, Sept. 1975, pp. $268-272$.

[40] H. Wheeler, "Transmission-Line Properties of Parallel Strips Separated by a Dielectric Sheet," IEEE Transactions on Microwave Theory and Techniques, vol. 13, no. 2, pp. 172 - 185, March 1965.

[41] M. Schneider, "Microstrip Lines for Microwave Integrated Circuits," The Bell System Technical Journal, vol. 48, no. 5, pp. 1421-1444, May/June 1969.

[42] E. Yamashita, K. Atsuki, and T. Hirahata, "Microstrip Dispersion in a Wide-Frequency Range," IEEE Transactions on Microwave Theory and Techniques, vol. 29, no. 6, pp. 610-611, June 1981.

[43] E. Yamashita, K. Atsuki, and T. Ueda, "An Approximate Dispersion Formula of Microstrip Lines for Computer-Aided Design of Microwave Integrated Circuits," IEEE Transactions on Microwave Theory and Techniques, vol. 27, no. 12, pp. 1036 - 1038, Dec. 1979.

[44] E. Yamashita and K. Atsuki, "Analysis of Microstrip-Like Transmission Lines by Nonuniform Discretization of Integral Equations," IEEE Transactions on Microwave Theory and Techniques, vol. 24, no. 4, pp. 195 - 200, Apr. 1976.

[45] M. Kirschning and R. Jansen, "Accurate model for effective dielectric constant of microstrip with validity up to millimetre-wave frequencies," Electronics Letters, vol. 18, no. 6, pp. $272-273$, March 1982.

[46] M. Kobayashi, "A dispersion formula satisfying recent requirements in microstrip CAD," IEEE Transactions on Microwave Theory and Techniques, vol. 36, no. 8, pp. 1246 - 1250, Aug. 1988.

[47] E. Hammerstad and O. Jensen, "Accurate Models for Microstrip Computer-Aided Design," in IEEE MTT-S International Microwave symposium Digest, May 1980, pp. 407 -409 .

[48] R. Pucel, D. Masse, and C. Hartwig, "Losses in Microstrip," IEEE Transactions on Microwave Theory and Techniques, vol. 16, no. 6, pp. 342 - 350, June 1968. 
[49] K. Gupta, R. Garg, and I. Bahl, Microstrip Lines and Slotlines, ser. Artech House Microwave Library. Artech House, 1979.

[50] N. Alexopoulos and S.-C. Wu, "Frequency-independent equivalent circuit model for microstrip open-end and gap discontinuities," IEEE Transactions on Microwave Theory and Techniques, vol. 42, no. 7, pp. 1268 -1272, July 1994.

[51] M. Kirschning, R. Jansen, and N. Koster, "Accurate model for open end effect of microstrip lines,” Electronics Letters, vol. 17, no. 3, pp. 123 -125, May 1981.

[52] E. Hammerstad and O. Jensen, "Accurate Models for Microstrip Computer-Aided Design," in IEEE MTT-S International Microwave symposium Digest, May 1980, pp. 407 -409 .

[53] P. Hammer, D. Van Bouchaute, D. Verschraeven, and A. Van de Capelle, "A model for calculating the radiation field of microstrip antennas," IEEE Transactions on Antennas and Propagation, vol. 27, no. 2, pp. 267 - 270, March 1979.

[54] "Data Sheet: WR-10 Series 27380 Matched Hybrid Tee," Flann Microwave Ltd, Dunmere Road Bodmin, Cornwall PL31 2QL United Kingdom.

[55] "Data Sheet: WR-10 Series 27060 Calibrated Phase Changer," Flann Microwave Ltd, Dunmere Road Bodmin, Cornwall PL31 2QL United Kingdom.

[56] “Data Sheet: WR-10 Series 27020 Calibrated Variable Attenuator," Flann Microwave Ltd, Dunmere Road Bodmin, Cornwall PL31 2QL United Kingdom.

[57] C. Wagner, A. Stelzer, and H. Jager, “A phased-array radar transmitter based on 77-GHz cascadable transceivers," in IEEE MTT-S International Microwave Symposium Digest, June 2009, pp. $73-76$.

[58] H.-Y. Chang, T.-W. Huang, H. Wang, Y.-C. Wang, P.-C. Chao, and C.-H. Chen, “A broadband HBT MMIC IQ modulator and millimeter-wave vector signal characterization," in MTT-S International Microwave Symposium Digest, vol. 1, June 2003, pp. 99 -102 vol.1.

[59] D. McPherson, I. Robertson, and S. Lucyszyn, "A W-band vector modulator and its application to software radar for automotive collision avoidance," in MTT-S International Microwave Symposium Digest, vol. 3, 2000, pp. 1423 -1426 vol.3.

[60] "Data Sheet: NI PXI-6229 Multifunction Data Acquisition Card," National Instruments, 11500 N Mopac Expwy Austin, TX 78759 USA.

[61] "Data Sheet: TSM-30 RF \& Microwave Laminate," Taconic, 136 Coonbrook Road Petersburgh, NY 12138 USA. 
[62] K. Sakakibara, M. Hirono, N. Kikuma, and H. Hirayama, "Broadband and planar microstrip-to-waveguide transitions in millimeter-wave band," in International Conference on ICMMT, Apr. 2008, pp. 1278-1281.

[63] Y.-C. Shih, T.-N. Ton, and L. Bui, "Waveguide-to-microstrip transitions for millimeterwave applications ," IEEE MTT-S International Microwave Symposium Digest, pp. 473475, May 1988.

[64] W. Grabherr, W. Huder, and W. Menzel, "Microstrip to waveguide transition compatible with mm-wave integrated circuits," vol. 42, no. 9, pp. 1842 -1843, sep 1994.

[65] L. Hyvonen and A. Hujanen, "A compact mmic-compatible microstrip to waveguide transition," in IEEE MTT-S International Microwave Symposium Digest, vol. 2, jun 1996, pp. $875-878$ vol.2.

[66] Z. Tong and A. Stelzer, "A vertical transition between rectangular waveguide and coupled microstrip lines," IEEE Microwave and Wireless Components Letters, vol. 22, no. 5, pp. $251-253$, May 2012.

[67] J.-H. Choi, K. Tokuda, H. Ogawa, and Y.-H. Kim, "Gap-coupled patch-type waveguideto-microstrip transition on single-layer dielectric substrate at V-band," Electronics Letters, vol. 40, pp. 1067 - 1068, 2004.

[68] S. Pandey and R. Ramadoss, "Coplanar patch antenna fed at the non-radiating edge," in IEEE Antennas and Propagation Society International Symposium, vol. 3, 2004, pp. 3397-3400 Vol.3.

[69] J. W. Kooi, G. Chattopadhyay et al., "A Full-Height Waveguide to Thin-Film Microstrip Transition with Exceptional RF Bandwidth and Coupling Efficiency," International Journal of Infrared and Millimeter Waves, vol. 24, no. 3, pp. 261-284, March 2003.

[70] F. Gustrau, $R F$ and Microwave Engineering: Fundamentals of Wireless Communications. Wiley, 2012.

[71] A. Tombak and A. Mortazawi, "A novel low-cost beam-steering technique based on the extended-resonance power-dividing method," IEEE Transactions on Microwave Theory and Techniques, vol. 52, no. 2, pp. 664-670, 2004. 


\section{A. Appendix}

In this thesis, a RO3003 laminate from Rogers Corporation is used as a dielectric substrate [30]. This material provides proved electrical and mechanical characteristics for $77 \mathrm{GHz}$ millimeter-wave automotive electronic systems. This laminate can be processed using standard low-cost PCB methods.

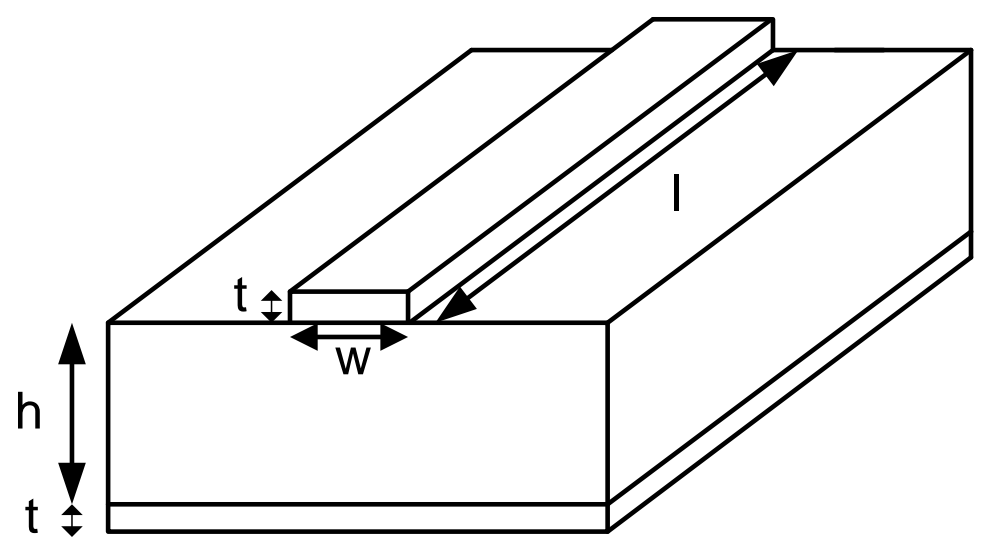

Figure A.1.: Physical parameters of an RF board

Table A.1.: Dimensions of a planar RF circuit on a RO3003 substrate

\begin{tabular}{l||l} 
Physical parameter & Value \\
\hline \hline Thickness of conductor $(\mathrm{t})$ & $0.018 \mathrm{~mm}$ \\
Thickness of ground plane $(\mathrm{t})$ & $0.018 \mathrm{~mm}$ \\
Thickness of substrate $(\mathrm{h})$ & $0.127 \mathrm{~mm}$ \\
Width of conductor $(50 \Omega$ line $)(\mathrm{w})$ & $0.32 \mathrm{~mm}$ \\
Dielectric permittivity $\left(\epsilon_{r}\right)$ & 3 \\
Dielectric loss $(\tan \delta)$ & $0.0013 @ 77 \mathrm{GHz}$ \\
Electrical conductivity $(\sigma)$ & $4.1 \times 10^{7} \mathrm{~S} / \mathrm{m}$
\end{tabular}




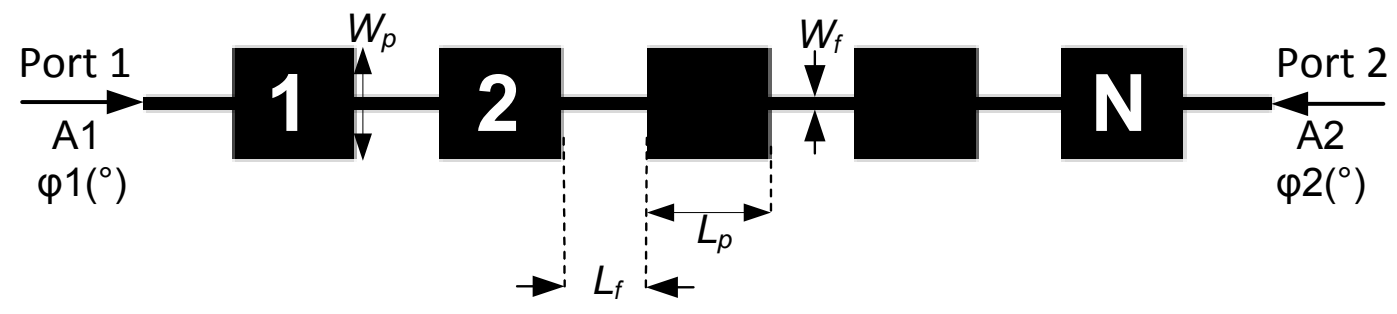

Figure A.2.: Layout of the designed linear array

Table A.2.: Optimized physical parameters of rectangular patches used for a linear array (Fig. A.2)

\begin{tabular}{l||l} 
Physical parameter & Value \\
\hline \hline Length of a patch $\left(L_{p}\right)$ & $1.09 \mathrm{~mm}$ \\
Width of a feed line $\left(W_{f}\right)$ & $0.1 \mathrm{~mm}$ \\
Width of a patch $\left(W_{p}\right)$ & $1.016 \mathrm{~mm}$
\end{tabular}




\begin{tabular}{|c|c|c|c|c|c|c|c|}
\hline \multicolumn{5}{|l|}{ Typical Values } & \multicolumn{3}{|c|}{ RO3000 ${ }^{\circledR}$ Series High Frequency Laminates } \\
\hline \multirow[t]{2}{*}{ PROPERTY } & \multicolumn{3}{|c|}{ TYPICAL VALUE (1) } & \multirow[t]{2}{*}{ DIRECTION } & \multirow[t]{2}{*}{ UNIT } & \multirow[t]{2}{*}{ CONDIION } & \multirow[t]{2}{*}{ TESTMEIHOD } \\
\hline & R03003 & RO3006 & RO3010 & & & & \\
\hline Dielectric Constant $\varepsilon_{\mathrm{r}}$ & $3.00 \pm 0.04^{(2)}$ & $6.15 \pm 0.15$ & $10.2 \pm 0.30$ & Z & - & $10 \mathrm{GHz} 23^{\circ} \mathrm{C}$ & $\begin{array}{c}\text { IPC }-T M-650 \\
2.5 .5 .5\end{array}$ \\
\hline Dissipation Factor & 0.0013 & 0.0020 & 0.0023 & Z & - & $10 \mathrm{GHz} 23^{\circ} \mathrm{C}$ & $\begin{array}{c}\text { IPC }-\mathrm{TM}-650 \\
2.5 .5 .5\end{array}$ \\
\hline $\begin{array}{l}\text { Thermal Coefficient } \\
\text { of } \varepsilon_{r}\end{array}$ & 13 & -160 & -280 & Z & $\mathrm{ppm} /{ }^{\circ} \mathrm{C}$ & $10 \mathrm{GHz} 0-100^{\circ} \mathrm{C}$ & $\begin{array}{l}\text { IPC-TM-650 } \\
2.5 .5 .5\end{array}$ \\
\hline Dimensional Stability & 0.5 & 0.5 & 0.5 & $X, Y$ & $\mathrm{~mm} / \mathrm{m}$ & CONDA & ASTM D257 \\
\hline Volume Resistivity & $10^{7}$ & $10^{3}$ & $10^{3}$ & & $\mathrm{M} \Omega \cdot \mathrm{cm}$ & CONDA & IPC 2.5.17.1 \\
\hline Surface Resistivity & $10^{7}$ & $10^{3}$ & $10^{3}$ & & $\mathrm{M} \Omega$ & CONDA & IPC 2.5.17.1 \\
\hline Tensile Modulus & $\begin{array}{l}2068 \\
(300)\end{array}$ & $\begin{array}{l}2068 \\
(300)\end{array}$ & $\begin{array}{l}2068 \\
(300)\end{array}$ & $X, Y$ & $\begin{array}{l}\mathrm{MPa} \\
\text { (kpsi) }\end{array}$ & $23^{\circ} \mathrm{C}$ & ASTM D638 \\
\hline Water Absorption & $\varangle 0.1$ & $\varangle 0.1$ & $\varangle 0.1$ & - & $\%$ & $\mathrm{D} 24 / 23$ & $\begin{array}{l}\text { IPC-TM-650 } \\
2.6 .2 .1 \\
\end{array}$ \\
\hline Specific Heat & $\begin{array}{c}0.93 \\
(0.22) \\
\end{array}$ & $\begin{array}{c}0.93 \\
(0.22) \\
\end{array}$ & $\begin{array}{c}0.93 \\
(0.22) \\
\end{array}$ & & $\begin{array}{c}\mathrm{J} / \mathrm{g} / \mathrm{K} \\
\left(\mathrm{BTU} / \mathrm{lb} /{ }^{\circ} \mathrm{F}\right) \\
\end{array}$ & & Calculated \\
\hline Thermal Conductivity & 0.50 & 0.61 & 0.66 & - & $\mathrm{W} / \mathrm{m} / \mathrm{K}$ & $100^{\circ} \mathrm{C}$ & ASTM C 518 \\
\hline $\begin{array}{l}\text { Coefficient of Thermal } \\
\text { Expansion }\end{array}$ & $\begin{array}{l}17 \\
24\end{array}$ & $\begin{array}{l}17 \\
24\end{array}$ & $\begin{array}{l}17 \\
24\end{array}$ & $\begin{array}{c}X, Y \\
Z\end{array}$ & $\mathrm{ppm} /{ }^{\circ} \mathrm{C}$ & -55 to $288^{\circ} \mathrm{C}$ & ASTM D3386-94 \\
\hline $\mathrm{Td}$ & 500 & 500 & 500 & & ${ }^{\circ} \mathrm{C}$ TGA & & ASTM D 3850 \\
\hline Color & Tan & Tan & Off White & & & & \\
\hline Density & 2.1 & 2.6 & 3.0 & & $\mathrm{gm} / \mathrm{cm}^{3}$ & & \\
\hline Copper Peel Strength & $\begin{array}{c}3.1 \\
(17.6)\end{array}$ & $\begin{array}{c}2.1 \\
(12.2)\end{array}$ & $\begin{array}{c}2.4 \\
(13.4)\end{array}$ & & $\begin{array}{l}\mathrm{N} / \mathrm{mm} \\
(\mathrm{lb} / \mathrm{in})\end{array}$ & $\begin{array}{l}\text { After solder } \\
\text { float }\end{array}$ & IPC-TM-2.4.8 \\
\hline Fla mma bility & $94 \mathrm{~V}-0$ & $94 \mathrm{~V}-0$ & $94 V-0$ & & & & UL \\
\hline $\begin{array}{l}\text { Lead-Free Process } \\
\text { Compatible }\end{array}$ & Yes & Yes & Yes & & & & \\
\hline
\end{tabular}

(1) References: Intemal T.R.'s 1430, 2224, 2854. Tests at $23^{\circ} \mathrm{C}$ unless otherwise noted. Typical values should not be used for specific ation limits.

(2) The nominal dielectric constant of an 0.060 " thick RO $3003^{\circledR}$ laminate as measured by the IPC-TM- $650,2.5 .5 .5$ will be 3.02 , due to the elimination of biasing caused by a ir gaps in the test fixture. For further information refer to Rogers T.R. 5242.

\begin{tabular}{|c|c|c|c|}
\hline \multicolumn{2}{|c|}{ STANDARD THIC KNESS: } & \multirow{2}{*}{$\begin{array}{l}\text { STANDARD PANEL SIZE: } \\
\text { RO3003: } \\
\text { 12" X 18" (305 X 457mm) } \\
\text { 24" X 18" (610 X 457mm) } \\
\text { 24" X 36" (610 X 915mm) }\end{array}$} & \multirow{2}{*}{$\begin{array}{l}\text { STANDARD COPPER CLADDING: } \\
1 / 2 \text { oz. }(17 \mu \mathrm{m}), 1 \text { oz. ( } 35 \mu \mathrm{m}), \\
2 \text { oz. }(70 \mu \mathrm{m}) \text { elec trodeposited copper foil. }\end{array}$} \\
\hline $\begin{array}{l}\text { RO3003: } \\
0.005^{\prime \prime}(0.13 \mathrm{~mm}) \\
0.010^{\prime \prime}(0.25 \mathrm{~mm}) \\
0.020^{\prime \prime}(0.50 \mathrm{~mm}) \\
0.030^{\prime \prime}(0.75 \mathrm{~mm}) \\
0.060^{\prime \prime}(1.52 \mathrm{~mm})\end{array}$ & $\begin{array}{l}\text { RO3006/3010: } \\
0.005^{\prime \prime}(0.13 \mathrm{~mm}) \\
0.010^{\prime \prime}(0.25 \mathrm{~mm}) \\
0.025^{\prime \prime}(0.64 \mathrm{~mm}) \\
0.050^{\prime \prime}(1.28 \mathrm{~mm})\end{array}$ & & \\
\hline
\end{tabular}

Figure A.3: Data sheet of the $\mathrm{RO} 3003$ dielectric substrate 